\title{
Regularity of the moving Fermi surface: RPA contributions
}

\section{Report}

Author(s):

Feldman, Joel S.; Salmhofer, Manfred; Trubowitz, Eugene

Publication date:

1997

Permanent link:

https://doi.org/10.3929/ethz-a-004353026

Rights / license:

In Copyright - Non-Commercial Use Permitted

Originally published in:

FIM's preprints 


\title{
Regularity of the Moving Fermi Surface: RPA Contributions
}

\author{
Joel Feldman $^{a, 1}$, Manfred Salmhofer $^{b, 2}$, And Eugene Trubowitz ${ }^{b, 3}$ \\ ${ }^{a}$ Mathematics Department, The University of British Columbia, Vancouver, Canada V6T $1 Z 2$ \\ ${ }^{b}$ Mathematik, ETH Zürich, CH-8092 Zürich, Switzerland
}

This paper is dedicated to the memory of Fritz John

\begin{abstract}
Regularity of the deformation of the Fermi surface under short-range interactions is established for all contributions to the RPA self-energy (it is proven in an accompanying paper that the RPA graphs are the least regular contributions to the self-energy). Roughly speaking, the graphs contributing to the RPA selfenergy are those constructed by contracting two external legs of a four-legged graph that consists of a string of bubbles. This regularity is a necessary ingredient in the proof that renormalization does not change the model. It turns out that the self-energy is more regular when derivatives are taken tangentially to the Fermi surface than when they are taken normal to the Fermi surface. The proofs require a very detailed analysis of the singularities that occur at those momenta $\mathbf{p}$ where the Fermi surface $S$ is tangent to $S+\mathbf{p}$. Models in which $S$ is not symmetric under the reflection $\mathbf{p} \rightarrow-\mathbf{p}$ are included.
\end{abstract}

\footnotetext{
1 feldman@math.ubc.ca, http://www.math.ubc.ca/ feldman/

2 manfred@math.ethz.ch, http://www.math.ethz.ch/ manfred/manfred.html

3 trub@math.ethz.ch
} 


\section{Introduction}

This paper is a continuation of [FST], which we refer to as I in what follows. Together with an accompanying paper, hereafter referred to as III, it contains the proof that the counterterm function of I is regular enough for the solution of the equations giving the renormalization of the Fermi surface (discussed in detail below) to exist. We shall recall briefly the motivation and the setting of the problem, as well as some results of I, to make this paper as self-contained as possible. For a detailed motivation, definitions of the scale flow and renormalization we refer the reader to I and to $[\mathrm{S}]$. All of this paper can be understood if one knows only the most elementary properties of scaled propagators, as stated in Lemma 2.3 of I. In particular, no familiarity with the formalism of the Gallavotti-Nicolò [GN] tree expansion is required.

\subsection{The Problem}

We consider a many-fermion system in a crystal background or on a lattice in $d \geq 2$ spatial dimensions, defined by a band structure and an interaction with a small coupling constant $\lambda$. The action for the model is

$$
\mathcal{A}=\int \frac{d^{d+1} p}{(2 \pi)^{d+1}} \bar{\psi}(p)\left(i p_{\mathrm{o}}-E(\mathbf{p})\right) \psi(p)+\lambda V(\psi, \bar{\psi})
$$

The basic assumptions we make are that the free band structure $E$ and the Fourier transform of the potential are both at least $C^{2}((\mathrm{H} 1)$ and $(\mathrm{H} 2))$, that the curvature of the Fermi surface $S$ is everywhere positive ((H3)), and, for $d=2$, that the filling factor is so small that certain umklapp processes do not happen in second order $((\mathrm{H} 5))$. For instance, in the two-dimensional Hubbard model, this restriction means that the filling factor $n$ has to obey $n<0.369$, where $n=1$ is half-filling. For $d \geq 3$, we need no filling restriction. We do not assume that $E(-\mathbf{p})=E(\mathbf{p})$ for all $\mathbf{p}$. If this symmetry does not hold, we call $E$ asymmetric. If $E$ is asymmetric, we also assume that the curvature at a point $\mathbf{p} \in S$ and at its antipode $\mathbf{a}(\mathbf{p})$ do not differ by too much $((\mathrm{H} 4))$, but that they coincide at only finitely many points $\left(\left(H 4^{\prime}\right)\right)$. We believe that all these assumptions are necessary and sufficient, that is, if one of them does not hold, not only the proofs, but also at least one conclusion of our theorems break down. The filling restriction (H5) is needed only in two dimensions, and only for special parts of our proofs.

The assumptions (H1)-(H5) are spelled out in detail in Chapter 2. They are stronger than those of I, so all results of I apply. The dynamics is given by the limit of the grand canonical ensemble as the volume tends to infinity and the temperature to zero. The interaction produces a self-energy of the electrons which one wants to calculate for small $\lambda$ by perturbation theory. The interaction may have drastic nonperturbative effects such as superconductivity, but in these weakly coupled systems, the correct way to begin the analysis, and to decide if this happens, is to study perturbation theory [FT1,FST].

It is well-known that the naive (unrenormalized) perturbation theory is infrared divergent because of the slow decay of the fermion two-point function in position space. The inherent slow decay is responsible 
for most of the physical (e.g. conductance) properties of these systems. In momentum space, this behaviour manifests itself as the singularity of the propagator on the Fermi surface, which, at zero temperature, is the boundary of the set of occupied states. As discussed in I, the infrared divergence of perturbation theory is not a problem of the model but of the way the expansion is done. Because of the self-energy effects, the Fermi surface moves when the interaction is turned on, and this motion of the singularity of the propagator causes the infrared divergences of a naive expansion. In I, we showed that, by making the band structure a function of $\lambda$, one can keep the Fermi surface fixed as the interaction is turned on, and thereby gave an infrared finite renormalized perturbation expansion. More precisely, for a given band structure $e$ and its associated covariance $\left(i p_{\mathrm{o}}-e(\mathbf{p})\right)^{-1}$, one can identify those parts $K(e, \lambda V, \mathbf{p})$ of the self-energy $\Sigma\left(p_{\mathrm{o}}, \mathbf{p}\right)$ that move the surface. Then the model with the modified band structure $e(\mathbf{p})+K(e, \lambda V, \mathbf{p})$ and interaction $\lambda V$ has a well-defined and locally Borel summable perturbation series. However, since

$$
K(e, \lambda V, \mathbf{p})=\sum_{r=1}^{\infty} K_{r}(e, V, \mathbf{p}) \lambda^{r}
$$

is a functional of $e$ and a function of the coupling $\lambda$ and the spatial part $\mathbf{p}$ of the momentum, simply replacing $e(\mathbf{p})$ by $e(\mathbf{p})+K(e, \lambda V, \mathbf{p})$ changes the model in a rather complicated way. To construct the original model with the given fixed free band relation $E$ and a given interaction one must solve $E=e+K$ for $e$. The free Fermi surface is the zero set of $E$, while the interacting Fermi surface is the zero set of $e$. The central problem is thus: fix a suitable $E$, and determine $e$ from

$$
E=e+K(e, \lambda V)
$$

That is, invert the map from the renormalized to the bare band structure. Once this is done, the counterterm function $K$ acquires a new role. It describes the deformation of the Fermi surface under the interaction. Thus proving regularity properties of $K$ as a function of $\mathbf{p}$, as we do here, is proving regularity of the moving Fermi surface.

In I, we proved uniqueness of the solution to (1.3) for a very large class of Fermi surfaces and interactions. This paper and its sequels are devoted to proving existence of the solution for the class of $E \in C^{2}$ which give rise to a strictly convex Fermi surface. We now discuss the main reasons why this is a rather nontrivial problem and give an outline how we solve it.

\subsection{Main Results}

It was explained in detail in [FST] why many terms in the unrenormalized perturbation expansion are divergent. In a nutshell, the coefficients in the series for e.g. the self-energy contain integrals over arbitrary powers of the free propagator $\left(i p_{\mathrm{o}}-e(\mathbf{p})\right)^{-1}$, which is not in $L^{2}$. For the same reason, it is far from trivial to prove any regularity of the self-energy and the counterterm function $K$ even after renormalization: every derivative increases the power of the denominator by one, and thus potentially introduces new divergences. Naive power counting suffices only to show Hölder continuity of degree $\alpha<1$ of the self-energy, which is not even sufficient to renormalize the theory. Moreover, it is evident that one will be able to solve (1.3) for generic $E \in C^{2}$ only if one can prove that $K$ is also a $C^{2}$ function of momentum. In I, we proved volume improvement estimates implying sharper power counting bounds that allow us to show that the theory can 
be renormalized and that the self-energy and the counterterm function are finite and $C^{1+\alpha}$ for some $\alpha>0$, determined by the Fermi surface geometry. We introduced the notion of overlapping loops (see Definition 2.19 of I) and showed that whenever a graph has overlapping loops, the integral for the phase space volume contains a subintegral bounded by

$$
\mathcal{W}(\varepsilon)=\sup _{\mathbf{q} \in \mathcal{B}} \max _{v_{i} \in\{ \pm 1\}} \int_{S^{d-1} \times S^{d-1}} d \theta_{1} d \theta_{2} \mathbb{1}\left(\left|e\left(v_{1} \mathbf{p}\left(0, \theta_{1}\right)+v_{2} \mathbf{p}\left(0, \theta_{2}\right)+\mathbf{q}\right)\right| \leq \varepsilon\right)
$$

(see $\mathrm{I},($ A.6)). Here $\mathcal{B}$ is the Brillouin zone, $\mathbb{1}(X)=1$ if $X$ is true, and zero otherwise. $\mathbf{p}(\rho, \theta)$ denotes a parametrization of a neighbourhood of the Fermi surface $S$ with $\rho=0$ corresponding to the Fermi surface itself. (This parametrization will be given in detail in Section 2.2. $\theta$ runs over $S^{d-1}$ because, under (H2)(H3), the Fermi surface is diffeomorphic to $S^{d-1}$ ). We showed in I that under a very general non-nesting condition, there is $0<\epsilon<1$ such that

$$
\mathcal{W}(\eta) \leq \text { const } \eta^{\epsilon}
$$

and that this implies volume-improved power counting bounds for all two-legged graphs and for all fourlegged graphs except for the ladder graphs. Any $\alpha<\epsilon$ can be used in the above statement that the self-energy is $C^{1+\alpha}$. Under the strict convexity condition imposed here, the bounds in I imply that

$$
\mathcal{W}(\varepsilon) \leq \operatorname{const} \varepsilon^{\frac{d-1}{d}}
$$

(see Proposition 1.1 and Lemma A.2 in I). In this paper, we prove

Theorem 1.1 Assume $(\mathrm{H} 2)_{2,0},(\mathrm{H} 3)$, and (H4). Then there is a constant $Q_{V} \geq 1$ such that for all $\varepsilon>0$

$$
\mathcal{W}(\varepsilon) \leq Q_{V} \varepsilon \begin{cases}|\log \varepsilon| & \text { if } d=2 \\ 1 & \text { if } d \geq 3\end{cases}
$$

The constant $Q_{V}$ depends only on $|e|_{2}=\sup _{\mathbf{p} \in \mathcal{B}} \sum_{|\alpha| \leq 2}\left|D^{\alpha} e(\mathbf{p})\right|$, and the numbers $r_{\mathrm{o}}, g_{\mathrm{o}}$, and $w_{\mathrm{o}}$ (defined in Chapter 2).

As mentioned, the precise assumptions $(H 1)-(H 5)$ are stated at the beginning of Chapter 2. Assumption (H5) is not needed in the proof of Theorem 1.1, which is given in Appendix B. In the application, $\varepsilon=M^{j}$ (with $M>1$ and $j<0$ ) is a small energy scale. The self-energy $\Sigma$ and the counterterm $K$ are given as scale sums, e.g.

$$
\Sigma(\mathbf{p})=\sum_{j<0} \Sigma^{(j)}(\mathbf{p})
$$

The standard power counting bound for this function is $\left|\Sigma^{(j)}\right| \leq M^{j}$, and every derivative multiplies this bound by a factor $M^{-j}$. In I we showed that actually $\left|\Sigma^{(j)}\right| \leq M^{j(1+\epsilon)}$, because of the extra volume improvement factor $M^{\epsilon j}$ coming from (1.5). This allows one to take almost $1+\epsilon$ derivatives and still have a convergent series in (1.8).

Theorem 1.1 implies that the volume improvement factor $M^{\epsilon j}$ of I can be replaced by $|j| M^{j}$ wherever it appears. Thus, the bound for the $\Sigma^{(j)}$ (and the similar bound for $K^{(j)}$ ) improves from the standard behaviour $M^{j}$ to $|j| M^{2 j}$ just by this volume estimate and Theorem $2.46(i)$ in I. Counting the effect of 
derivatives in the way described, we see that the scale sum over $I<j<0$ of the second derivative of $K$ or $\Sigma$ diverges at most as $|I|^{2}$, not as $M^{|I|}$, as $I \rightarrow-\infty$, as unimproved power counting would suggest. In other words, Theorem 1.1 ensures that the divergence of the second derivative can be, at worst, marginal and one may hope for convergence with a little more care in doing the bounds.

The volume bound stated above is best possible, however, and so one has to go beyond volume estimates even to solve the problem in second order. It turns out that this requires a much more detailed analysis. Because the Fermi surface is a $d$-1-dimensional submanifold of $d$-dimensional space, there are angular variables parametrizing it, and which also occur as integration variables. The idea is now to use these angular integration variables to prevent derivatives from degrading the scale behaviour, by moving the dependence on the external momentum from propagators $\frac{1}{i p_{\mathrm{o}}-e(\mathbf{p})}$ into a Jacobian. However, this Jacobian has singularities whose location depends on the external momentum. For the second order contribution, we do a careful analysis of these singularities and show that under our hypotheses, in particular because the Fermi surface has strictly positive curvature, the second order counterterm has exactly the same degree of differentiability as $e$.

\section{Theorem 1.2}

(i) Let $d=2$. There exists $h>0$ such that if $0 \leq h^{\prime} \leq h, k \geq 2$, and $(\mathrm{H} 1)_{k, h^{\prime}},(\mathrm{H} 2)_{k, h^{\prime}},(\mathrm{H} 3)-(\mathrm{H} 5)$ hold, then $K_{2}(e, V, \mathbf{p})$, given in $(1.2)$, is $C^{k, h^{\prime}}$ in $\mathbf{p}$. Moreover, the second order self-energy $\Sigma_{2}$ is $C^{1, \gamma}$ in $\mathbf{p}$ for any $\gamma \in(0,1)$.

(ii) Let $d \geq 3$. There exists $h>0$ such that if $0 \leq h^{\prime} \leq h$, and $(\mathrm{H} 1)_{2, h^{\prime}},(\mathrm{H} 2)_{2, h^{\prime}},(\mathrm{H} 3)$, and (H4) hold, then $K_{2}$ and $\Sigma_{2}$ are $C^{2, h^{\prime}}$ in $\mathbf{p}$.

Here $C^{k, h}$ is the set of functions all of whose $k^{\text {th }}$ order derivatives are Hölder continuous of index $h . h^{\prime}=0$ is allowed.

Note that Theorem 1.2 states for $d=2$ that if $e$ and $\hat{v}$ are $C^{2}$, then the counterterm $K_{2}$ is also $C^{2}$, whereas the self-energy $\Sigma_{2}$ is only shown to be $C^{1, \gamma}$ for any $\gamma<1$, (that is, more loosely speaking, $\Sigma_{2} \in C^{2-\varepsilon}$ for any $\varepsilon>0$ ), even if $e \in C^{k}$ and $\hat{v} \in C^{k}$ for some $k \geq 2$. We can prove convergence of the second derivative of the second-order self-energy $\Sigma_{2}$ in two dimensions only if the derivative is taken tangential to $S$. For derivatives taken in the direction $\rho$ transversal to $S$, or with respect to $p_{\mathrm{o}}$, we show that in $d=2$, the second derivative is at most logarithmically divergent. The calculations in $[\mathrm{F}]$ indicate that this logarithm is indeed there in two dimensions, i.e. that

$$
\Sigma_{2}\left(p_{\mathrm{o}}, \mathbf{p}\right) \sim p_{\mathrm{o}}^{2} \log \left|p_{\mathrm{o}}\right| .
$$

Stated differently, at positive temperature $T>0$ (which provides a natural infrared cutoff), the behaviour of $\Sigma_{2}$ is $T^{2} \log T$. The extra logarithm played a role in the discussion about the existence of two-dimensional Fermi liquids $[F]$. Our results imply that this logarithmic singularity is (if it exists) not an obstruction to the perturbative solution of (1.3), because we are doing renormalization using the more regular function $K$ instead of $\Sigma$ itself. At present we do not know a way of making the skeleton expansion, where one subtracts $\Sigma$, not only $K$, rigorous in $d=2$. We explain these problems further in the next section.

The statement that $\Sigma \in C^{1, \gamma}$ for any $\gamma<1$ also holds for the full perturbative self-energy, by Theorem 1.1. We did not state it in Theorem 1.2 because the proof, although an easy combination of Theorem 2.46 of I and the methods developed in Section 3.4, requires familiarity with the tree expansion. We shall give it in III. 
As stated in Theorem 1.2, in three dimensions, the self-energy is $C^{2}$ in $\mathbf{p}$, so there is no logarithm in the second derivative with respect to $\rho$. Thus for $d \geq 3$, the skeleton expansion can be made rigorous by our results. One can also combine the methods of Chapter 3 of I and the ones developed here to show that in the $p_{\mathrm{o}}$-dependence of $\Sigma$, the logarithm of (1.9) is absent in three dimensions, i.e., that $\Sigma$ is $C^{2}$ also in $p_{0}$.

Although elementary, our detailed analysis of the second order counterterm is rather subtle, and a generalization to all two-legged 1PI graphs (those contributing to $\Sigma$ and $K$ ) gives a system of equations for the singularities that looks rather hopeless. Fortunately, a generalization to all two-legged 1PI graphs is not required. In III, we give a new graph classification which isolates the only graphs that require the detailed analysis done here, and that can exhibit behaviour as in (1.9) for $d=2$. These graphs constitute, in a sense to be specified later, the (generalized) random-phase approximation contributions $\Sigma_{\mathrm{RPA}}, K_{\mathrm{RPA}}$ to the self-energy and the counterterm. See Chapter 4. For these graphs, a detailed analysis of singularities is possible and we show

Theorem 1.3 Let $\Sigma_{\mathrm{RPA}}$ be the RPA self-energy and $K_{\mathrm{RPA}}$ the RPA counterterm.

(i) If $d=2$ and $e(-\mathbf{p})=e(\mathbf{p})$ for all $\mathbf{p}$, there exists $h>0$ such that if $0 \leq h^{\prime} \leq h, k \geq 2$ and if $(\mathrm{H} 1)_{k, h^{\prime}},(\mathrm{H} 2)_{k, h^{\prime}},(\mathrm{H} 3)$, and $(\mathrm{H} 5)$ hold, then $K_{\mathrm{RPA}}$ is a $C^{k, h^{\prime}}$ function of $\mathbf{p} . h^{\prime}=0$ is allowed.

(ii) If $d=2$, there exists $h>0$ such that if $0<h^{\prime} \leq h$ and if $(\mathrm{H} 1)_{2, h^{\prime}},(\mathrm{H} 2)_{2, h^{\prime}},(\mathrm{H} 3)-(\mathrm{H} 5)$ and $\left(\mathrm{H} 4^{\prime}\right)$ hold, then $K_{\mathrm{RPA}}$ is $C^{2, h^{\prime}}$ in $\mathbf{p}$. In this case, the condition $e(-\mathbf{p})=e(\mathbf{p})$ for all $\mathbf{p}$ would force a violation of (H4'). $h^{\prime}=0$ is not allowed.

(iii) If $d \geq 3$, there exists $h>0$ such that if $0 \leq h^{\prime} \leq h$ and $(\mathrm{H} 1)_{2, h^{\prime}},(\mathrm{H} 2)_{2, h^{\prime}}$, and (H3) (and, for asymmetric e, (H4)) hold, then $K_{\mathrm{RPA}}$ and $\Sigma_{\mathrm{RPA}}$ are $C^{2, h^{\prime}}$ in $\mathbf{p} . h^{\prime}=0$ is allowed.

Note that, as in Theorem 1.2, the self-energy is shown to be $C^{2}$ in $d \geq 3$. In two dimensions, one expects (both from the bounds we derive and calculations in the literature) that RPA graphs also produce a behaviour as in (1.9). Note also that for asymmetric $e$, we only prove $K \in C^{2}$, not $C^{k}$ with $k \geq 3$. The detailed analysis done in Chapter 4 suggests that the third derivative of $K_{\mathrm{RPA}}$ may actually not converge if $e$ is asymmetric.

The extension of Theorems 1.2 and 1.3 to the exact self-energy and the exact $K$ is proven in III.

Thus, in any fixed order $r$ of perturbation theory, we can start to look for solutions of (1.3) by iteration. The uniqueness theorem proven in I then guarantees that if the iterative sequence has an accumulation point, it converges. But our bounds are good enough to prove convergence directly, so that one has a solution of (1.3).

\subsection{Consequences}

To put these results into context, we now discuss how one gets from the solution of (1.3) to a rigorous version of what is usually called 'self-consistent renormalization'. Given a free model with band structure $E$ (including the chemical potential $\mu$ ), we want to do a formal power series expansion expansion in $\lambda$ for 
the interacting model given by the generating functional for connected amputated Green functions

$$
e^{\mathcal{G}(\psi, \bar{\psi})}=\frac{1}{Z_{E}} \int d \mu_{C_{E}}(\chi, \bar{\chi}) e^{-\lambda V(\psi+\chi, \bar{\psi}+\bar{\chi})}
$$

with $d \mu_{C_{E}}$ the Grassmann Gaussian measure with propagator $1 /\left(i p_{\mathrm{o}}-E(\mathbf{p})\right)$. The constant $Z_{E}$ normalizes $\mathcal{G}(0,0)=0$. This may be defined as the limit $\beta \rightarrow \infty$ of the grand canonical ensemble with partition function $\operatorname{tr} e^{-\beta(H-\mu N)}$. Denote the solution to equation (1.3) by $e=F(E, \lambda)$. Let $\kappa(E, \lambda)=K(F(E, \lambda), \lambda)$. Then $E=e+\kappa=e+K(e, \lambda)$. We now move $K$ from the Gaussian measure into the interaction by the standard Gaussian shift formula, so that $K$ now acts as a counterterm. Since $E=e+K$, this leaves $e$ in the propagator, and

$$
\begin{aligned}
e^{\mathcal{G}(\psi, \bar{\psi})} & =\frac{1}{Z_{e}} \int d \mu_{C_{e}}(\chi, \bar{\chi}) e^{-(\bar{\chi}, K \chi)} e^{-\lambda V(\psi+\chi, \bar{\psi}+\bar{\chi})} \\
& =\frac{1}{Z_{e}} e^{-(\bar{\psi}, K \psi)} \int d \mu_{C_{e}}(\chi, \bar{\chi}) e^{-(\bar{\psi}+\bar{\chi}, K(\psi+\chi))} e^{-\lambda V(\psi+\chi, \bar{\psi}+\bar{\chi})} e^{(\bar{\psi}, K \chi)+(\bar{\chi}, K \psi)} .
\end{aligned}
$$

The change in normalization factor from $Z_{E}$ to $Z_{e}$ is irrelevant for any correlation function, and the extra source terms in the integrand just modify the external legs in a trivial way. Effectively, external vertices are not renormalized. Because e is given by the solution to (1.3), the model has not been changed in any way. What has changed is our way of looking at it. Splitting $E=e+\kappa$ means going from the bare to the interacting Fermi surface. After that, the other interaction effects can be calculated at fixed surface. In fact, everything is already arranged such that the expansion of (1.11) in $\lambda$ is precisely the renormalized expansion of I. All theorems of I apply. In particular, there are no infrared divergences.

In other words, the interaction effects are calculated in two steps: first, we determine the interacting Fermi surface, then the self-energy and the $n$-point functions.

It is often stated that the renormalization problem can be dealt with by doing a skeleton expansion in which on all lines, the free propagator is replaced by the interacting propagator (sometimes, this is also called 'self-consistent renormalization'). That is, one calculates the values of skeleton diagrams, using for the $r^{\text {th }}$ order in $\lambda$ the interacting propagator $\left(i p_{o}-e(\mathbf{p})-\Sigma_{r-1}(p)\right)^{-1}$, where $\Sigma_{r-1}$ is the self-energy up to order $r-1$ in $\lambda$, instead of the free propagator $\left(i p_{0}-e(\mathbf{p})\right)^{-1}$. However, a regularity problem similar to (1.3) also arises in this procedure: to show that the values of skeleton diagrams with propagators containing $e+\Sigma$ are well-defined, one has to assume that $\Sigma$ has the same regularity properties as $e$. However, $\Sigma$ is not a function one is free to choose. One has to show regularity of the self-energy. This regularity problem is harder than the one necessary to invert (1.3), because $\Sigma$ is even less regular than $K$, and in fact, it may not have a solution in $d=2$ dimensions. Let us be more specific about why regularity of $\Sigma$ (or $K$ ) is needed. To show that the most elementary power counting estimates hold, one needs that the volume of a shell of thickness $\varepsilon$ around the Fermi surface is bounded by a constant times $\varepsilon$. This can be shown for the surface $S(e)=\{\mathbf{p}: e(\mathbf{p})=0\}$ if $e \in C^{1}$, and if the gradient of $e$ does not vanish on $S(e)$ (see (H2)). To have the same statement for the surface $e(\mathbf{p})+\Sigma(0, \mathbf{p})=0$, one also has to show that $\Sigma(0, \mathbf{p})$ is $C^{1}$ in $\mathbf{p}$. But to show that $\Sigma$ is $C^{1}$, one needs upper and lower bounds on the curvature of $S(e)$ - which requires $e \in C^{2}-$ already in second order (if the Fermi surface has flat sides, or if the system is one-dimensional, $\Sigma$ is typically not $C^{1}$ ). Since the second order $\Sigma$ appears on 'interacting propagators' of higher order graphs, one needs $\Sigma \in C^{2}$. It was mentioned above that most likely, $\Sigma \notin C^{2}$, because of the logarithm in (1.9), so proceeding this way may be impossible. Instead we take a counterterm function $K$ which agrees with $\Sigma$ only on the Fermi surface. Tangential derivatives of $K$ agree with tangential derivatives of $\Sigma$, but normal derivatives do 
not agree. A priori, proving $K \in C^{2}$ could require $e \in C^{3}$, and, hence in the next order $K \in C^{3}$, and so on. However, this sequence stops already at $k=2$ : we show that $e \in C^{2}$ implies $K \in C^{2}$. This is equivalent to saying that the iteration for the solution of (1.3) stays in a fixed set of $C^{2}$ band structures.

In renormalization group studies, a wave function renormalization is often introduced. For instance, in one-dimensional systems, it is crucial for taking into account the anomalous dimension correctly [BGPS,BM]. In dimension $d \geq 2$, our results show that a wave function renormalization is not necessary for renormalizing formal perturbation theory, although it may be convenient. One can easily retrieve the perturbative wave function renormalization, and show that it is finite to all orders, in the usual way from the self-energy: we proved in I that $\Sigma \in C^{1}$. Moreover, our assumption that $\hat{v}\left(-p_{0}, \mathbf{p}\right)=\overline{\hat{v}\left(p_{0}, \mathbf{p}\right)}$ (see (H1)) implies that $\Sigma\left(-p_{\mathrm{o}}, \mathbf{p}\right)=\overline{\Sigma\left(p_{\mathrm{o}}, \mathbf{p}\right)}$, so that $\Sigma(0, \mathbf{p}) \in \mathbb{R}$ for all $\mathbf{p}$. Since $\Sigma$ is $C^{1}$ in $p$, this implies $\left(\partial_{\mathrm{o}} \Sigma\right)(0, \mathbf{p}) \in i \mathbb{R}$ and $\nabla \Sigma(0, \mathbf{p}) \in \mathbb{R}$ for all $\mathbf{p}$. Recalling that by Theorem 1.2 (iii) of I, $\Sigma(0, \mathbf{P}(\mathbf{p}))=0$ (here $\mathbf{P}$ denotes the projection onto the Fermi surface), we get by Taylor expansion

$$
\Sigma(p)=p_{0}\left(\partial_{0} \Sigma\right)(0, \mathbf{P}(\mathbf{p}))+(\mathbf{p}-\mathbf{P}(\mathbf{p})) \cdot \nabla \Sigma(0, \mathbf{P}(\mathbf{p}))+\tilde{\Sigma}(p)
$$

where $\tilde{\Sigma}$ vanishes faster than linear as $p_{0}$ approaches 0 and $\mathbf{p}$ the Fermi surface $S$. By the result that $\Sigma \in C^{1, \gamma}$ for all $\gamma<1$, we know that $\tilde{\Sigma}$ vanishes almost quadratically in that limit. Combining the linear term in $p_{\mathrm{o}}$ with the $i p_{\mathrm{o}}$ of the free propagator, we get the usual formula

$$
Z(\mathbf{p})=1+i\left(\partial_{\mathrm{o}} \Sigma\right)(0, \mathbf{P}(\mathbf{p})) \in \mathbb{R}
$$

for the prefactor of $i p_{\mathrm{o}}$ (whether one calls $Z$ or $1 / Z$ the wave function renormalization is, of course, a matter of convention). The $\nabla \Sigma$ term gives the usual correction to the Fermi velocity. As mentioned above, the shift from the free to the interacting Fermi surface is given by $K(\mathbf{p})$.

What does this perturbative analysis imply for the full, nonperturbative model? This depends on $e$. If $e(-\mathbf{p})=e(\mathbf{p})$, the perturbation series is not convergent, and thus defines only formal power series, because of the factorial growth of the contribution of the ladder diagrams. It is well-known that the particle-particle ladder graphs really produce such factorials if $e(-\mathbf{p})=e(\mathbf{p})$. It is proven very generally in I that for many-fermion models, only the ladders can produce those factorials. Thus there are no other graphs whose contribution could cancel them, and they prevent convergence of the renormalized perturbation series. (Do not confuse this with the infrared divergences discussed above. In the renormalized expansion $\Sigma=\sum \lambda^{r} \Sigma_{r}$, all $\Sigma_{r}$ are finite $C^{1}$ functions, but the convergence radius of the power series in $\lambda$ is zero). An improvement of power counting (called loop improvement), and the corresponding statement that the ladders give the only singular contribution to the four-point function was also stated in [BG] for the case where the Fermi surface is a sphere. It is proven in [FMRT] by implementing the Pauli principle that at least for spatial dimension $d=2$ there are no other obstacles to the convergence of the renormalized perturbation series. It is proven in [FT2] that for an attractive interaction, the perturbative RG flow, which is dominated by the ladder diagrams, leads to a symmetry-breaking fixed point given by Cooper pairing.

All this changes very much for the class of $e$ which violates the symmetry $e(-\mathbf{p})=e(\mathbf{p})$ (the precise class is specified in (H4) and (H4')), to which a big part of our analysis is devoted. This asymmetry suppresses the Cooper instability, i.e. it removes the factorial growth of the ladders. By the result proven in I that the contribution from all other graphs to the four-point function is nonsingular, one may suspect that perturbation theory converges. It is proven nonperturbatively in [FKLT] in $d=2$ that the renormalized perturbation expansion converges in these models. Thus models with $e(-\mathbf{p}) \neq e(\mathbf{p})$ can be proven to be 
Fermi liquids. The results of I and of the present paper provide the perturbative part of this proof: in I we have shown that the renormalized Green functions are all finite, and that only ladder subdiagrams produce factorial growth in the value of individual diagrams. In the present paper and III, we prove that renormalization does not change the model.

While differentiability of the self-energy may look like a rather technical problem at first sight, we should like to remark that a self-energy that is not $C^{1}$ is a common feature of various proposals for nonFermi-liquid behaviour in two dimensions (see $[\mathrm{S}]$ for further discussion), so that the differentiability issue does have a physical significance.

We end this introduction with some more detailed remarks. As anybody who actually reads the proofs will see, being in a $C^{2}$ (or $C^{2, h}$ ) class of functions poses some rather severe technical restrictions, which show up in various technical details. For instance, the proof of Theorem 1.1 requires a version of the Morse lemma for $C^{2}$ functions. Since this lemma is not completely standard, we prove it in Appendix A. Moreover, the critical point analysis involves the antipode $\mathbf{a}(\mathbf{p})$ of $\mathbf{p} \in S$, which is the point on $S$ where $\nabla e(\mathbf{a}(\mathbf{p}))$ is antiparallel to $\nabla e(\mathbf{p})$. For a $C^{k}$ surface, $\mathbf{a}$ is in general only a $C^{k-1}$ function. It requires careful arguments to show that this does not cause a deterioration of the differentiability properties of $K$.

To do renormalization without changing the model, we may use the renormalized expansion only after inverting (1.3). This restricts us to a class of $C^{2, h}$ functions even if the starting $E$ had a higher degree of differentiability, because it is the differentiability of $e$ that enters the bounds. One consequence of this is that the higher-tangency-argument used in [FKLT] to show the absence of the Cooper instability (i.e., the boundedness of the particle-particle ladders) does not apply. We define a notion of minimal change of the curvature (similar to the definitions in $\mathbf{A 3}$ of I) for our class of $C^{2, h}$ functions in Assumption (H4') and show in Appendix $\mathrm{C}$ that the particle-particle ladders are bounded under this weaker condition. We also need this for the regularity proofs. It is at this point that we actually need the extra Hölder continuity stated in the above Theorems. In particular, it is the reason why $h^{\prime}=0$ is not allowed in Theorem $1.3(i i)$. In a $C^{2}$ class of functions, a natural definition of a minimal change in curvature would be impossible.

Finally, we note that although the filling restriction (H5) that we imposed for $d=2$ may seem peculiar, numerical results indicate that in its absence, the behaviour of the self-energy is indeed different. These effects may also be of physical interest.

We give the definition of our class of models in Chapter 2 and prove Theorem 1.2 in Chapter 3 , and Theorem 1.3 in Chapter 4. Appendix A contains the $C^{2}$ Morse Lemma, and Appendix B contains the proof of Theorem 1.1. Appendix $\mathrm{C}$ contains the proof of the one-loop volume bound needed to prove Theorem 1.3 in case $e$ is asymmetric. In Appendix D, we prove regularity properties of the scale zero effective action, which relates the theory without a cutoff in $p_{\mathrm{o}}$ to the one with a cutoff. 


\section{Definitions and Assumptions}

We denote the $\varepsilon$-neighbourhood of a set $A$

$$
U_{\varepsilon}(A)=\{y: \exists x \in A \text { with }|y-x|<\varepsilon\}
$$

and, as in I, we denote the norm

$$
|f|_{k}=\sup _{p \in \mathbb{R} \times \mathcal{B}} \sum_{|\alpha| \leq k}\left|D^{\alpha} f(p)\right|
$$

where $\alpha=\left(\alpha_{\mathrm{o}}, \ldots, \alpha_{d}\right) \in \mathbb{Z}^{d+1}, \alpha_{i} \geq 0$ for all $i$, is a multiindex, $|\alpha|=\sum_{i=0}^{d} \alpha_{i}$, and $\partial^{\alpha}=\left(\frac{\partial}{\partial p_{\mathrm{o}}}\right)^{\alpha_{\mathrm{o}}} \ldots\left(\frac{\partial}{\partial p_{d}}\right)^{\alpha_{d}}$.

Let $0<h \leq 1$. We denote the space of $C^{k}$ functions on a set $\Omega$ whose $k^{\text {th }}$ derivative is $h$-Hölder continuous by $C^{k, h}(\Omega)$, and use the norm

$$
|f|_{k, h}=\sup _{p \in \mathbb{R} \times \mathcal{B}} \sum_{|\alpha| \leq k}\left|D^{\alpha} f(p)\right|+\max _{\alpha:|\alpha|=k} \sup _{\substack{x, y \in \Omega \\ x \neq y}} \frac{\left|D^{\alpha} f(x)-D^{\alpha} f(y)\right|}{|x-y|^{h}} .
$$

For $h=0$, we define $C^{k, 0}(\Omega)=C^{k}(\Omega)$.

\subsection{Band structure and interaction}

Our analysis takes place in momentum space, given by $\mathbb{R} \times \mathcal{B}$, where $\mathcal{B}$ is the torus $\mathbb{R}^{d} / \Gamma^{\#}$, with $\Gamma^{\#}$ the dual lattice to the position space lattice $\Gamma$, e.g. for $\Gamma=\mathbb{Z}^{d}, \mathcal{B}=\mathbb{R}^{d} / 2 \pi \mathbb{Z}^{d}$. Let $\mathcal{F} \subset \mathbb{R}^{d}$ be a fundamental domain for the action of the translation group $\Gamma^{\#}$, and $\stackrel{\mathcal{F}}{\subset} \subset \mathbb{R}^{d}$ its interior, e.g. for $\Gamma^{\#}=2 \pi \mathbb{Z}^{d}$, and $\mathcal{B}=\mathbb{R}^{d} / 2 \pi \mathbb{Z}^{d}$, $\mathcal{F}=[-\pi, \pi)^{d}$ and $\mathcal{F}^{\circ}=(-\pi, \pi)^{d}$. We could also take $\mathcal{B}$ to be a bounded subset of $\mathbb{R}^{d}$.

The one-electron problem provides a band structure $e(\mathbf{p})$ which enters the propagator associated to lines $\longrightarrow$. We include the chemical potential $\mu$ in $e$. For example, for the free electron gas, $e(\mathbf{p})=\frac{\mathbf{p}^{2}}{2 m}-\mu$. The interaction is given by the vertex

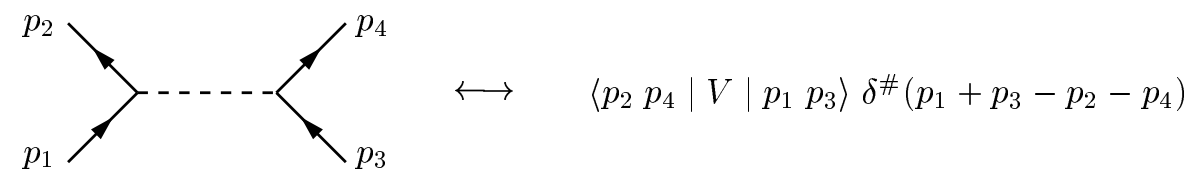

where $\delta^{\#}$ is a $\delta$ function on $\mathbb{R}$ for $p_{\text {o }}$ and a $\delta$ function on $\mathcal{B}$ (i.e. one on $\mathbb{R}^{d}$ modulo $\Gamma^{\#}$ ) for the spatial part $\mathbf{p}$. The vertex function has the symmetry

$$
\left\langle p_{2} p_{4}|V| p_{3} p_{1}\right\rangle=\left\langle p_{4} p_{2}|V| p_{1} p_{3}\right\rangle .
$$


For simplicity, we assume that

$$
\left\langle p_{2} p_{4}|V| p_{1} p_{3}\right\rangle=\hat{v}\left(p_{2}-p_{1}\right) .
$$

We make the following assumptions on the interaction $\hat{v}$, the band structure $e(\mathbf{p})$ and the Fermi surface $S=\{\mathbf{p} \in \mathcal{B}: e(\mathbf{p})=0\}$. For some $k \geq 2$,

$(\mathrm{H} 1)_{k, h} \quad \hat{v} \in C^{k, h}(\mathbb{R} \times \mathcal{B}, \mathbb{C})$ with all derivatives of order at most $k$ uniformly bounded on $\mathbb{R} \times \mathcal{B}$, and $\hat{v}$ satisfies

$$
\hat{v}\left(-p_{\mathrm{o}}, \mathbf{p}\right)=\overline{\hat{v}\left(p_{\mathrm{o}}, \mathbf{p}\right)} .
$$

There is a bounded real-valued function $\tilde{v} \in C^{k, h}(\mathcal{B}, \mathbb{R})$ such that for all $\mathbf{p} \in \mathcal{B}$

$$
\lim _{p_{\mathrm{o}} \rightarrow \infty} \hat{v}\left(p_{\mathrm{o}}, \mathbf{p}\right)=\tilde{v}(\mathbf{p})
$$

and there are $\alpha>0, K_{\mathrm{o}}>0$, and $\pi_{\mathrm{o}}>0$ such that

$$
\forall\left|p_{\mathrm{o}}\right| \geq \pi_{\mathrm{o}} \forall \mathbf{p} \in \mathcal{B}: \quad\left|\hat{v}\left(p_{\mathrm{o}}, \mathbf{p}\right)-\tilde{v}(\mathbf{p})\right| \leq K_{\mathrm{o}}\left|p_{\mathrm{o}}\right|^{-\alpha}
$$

(2.6) implies that the counterterms $K$, defined below, are real-valued, $K(\mathbf{p}) \in \mathbb{R}$ for all $\mathbf{p} \in \mathcal{B}$. (2.6) and (2.7) also imply that $\lim _{p_{\mathrm{o}} \rightarrow-\infty} \hat{v}\left(p_{\mathrm{o}}, \mathbf{p}\right)=\tilde{v}(\mathbf{p})$.

The decay condition (2.8) assures us that the scale zero effective action, defined below, gives rise to $C^{k, h}$ vertex functions, and that therefore the ultraviolet (large $p_{\mathrm{o}}$ ) part of the problem can be separated from the infrared (small $p_{\mathrm{o}}$ ) part by the semigroup structure of the flow of effective actions. If the interaction is instantaneous, i.e., if $\hat{v}$ is independent of $p_{\mathrm{o}},(2.8)$ holds trivially.

$$
(\mathrm{H} 2)_{k, h} \quad e \in C^{k, h}(\mathcal{B}, \mathbb{R}) \text { and } \nabla e(\mathbf{p}) \neq 0 \text { for all } \mathbf{p} \in S
$$

Assumption (H2) implies that $S$ is a $C^{k}$ submanifold of $\mathcal{B}$. Since $k \geq 2$, its curvature $\kappa$ is therefore welldefined. Since $\mathcal{B}$ is compact, $e$ is continuous, and $S=e^{-1}(\{0\})$ is a preimage of zero, $S$ is compact.

$$
\kappa \text { is strictly positive everywhere. }
$$

In $d>2$ this is meant in the matrix sense. (H3) implies that $S$ bounds a strictly convex set. In two dimensions, $S$ is a simple closed curve. Let $\mathbf{n}=\frac{\nabla e}{|\nabla e|}$ be the unit normal to $S$. Strict convexity implies that the equation

$$
\mathbf{n}(\mathbf{a}(\mathbf{p}))=-\mathbf{n}(\mathbf{p})
$$

has, for any $\mathbf{p} \in S$, a unique solution $\mathbf{a}(\mathbf{p}) \in S$. Necessarily, $\mathbf{a}(\mathbf{p}) \neq \mathbf{p}$. We call $\mathbf{a}(\mathbf{p})$ the antipode of $\mathbf{p}$. Since $\mathbf{n}$ is $C^{k-1}, \mathbf{a} \in C^{k-1}(S, S)$.

We shall not assume in general that $e(-\mathbf{p})=e(\mathbf{p})$. We call a band structure $e$ symmetric if

$$
\text { For all } \mathbf{p} \in \mathcal{B}, e(-\mathbf{p})=e(\mathbf{p}) \text {. }
$$

and asymmetric otherwise. If $(S y)$ holds, $\mathbf{a}(\mathbf{p})=-\mathbf{p}$. In the asymmetric case, we assume 


$$
\left.|1-| \frac{\partial \mathbf{p}(t)}{\partial t}\right|^{-1}\left|\frac{\partial \mathbf{a}(\mathbf{p}(t))}{\partial t}\right| \mid \leq \frac{1}{8}
$$

If $e$ is symmetric, (H4) holds trivially by (Sy) (the left hand side of (2.10) is zero). We shall give a restatement of (H4) in terms of the curvature below. Essentially, the curvature at the points $\mathbf{p}$ and $\mathbf{a}(\mathbf{p})$ must not differ by too much.

For asymmetric $e$ and $d=2$, we need another assumption, (H4'), which we state after we have introduced coordinates, because it is more easy to state, and understand, after some preparations. This assumption forces the curvature at almost all points $\mathbf{p}$ on the Fermi surface to differ from the curvature at the antipode $\mathbf{a}(\mathbf{p})$.

The last assumption is

$$
\{u \mathbf{p}+v \mathbf{q}: \mathbf{p}, \mathbf{q} \in S, u, v= \pm 1\} \subset \stackrel{\circ}{\mathcal{F}} .
$$

Assumptions (H1) and (H2) are identical to the assumptions $\mathbf{A 1}$ and A2 of I, save for the condition (2.8) on $\hat{v}$ which we use for the ultraviolet part of the problem (which was discarded in I), and the extra (optional) Hölder continuity. They are satisfied for interactions that decay fast enough, and under natural conditions on the one-particle problem. For a detailed discussion of their meaning, see Section 1.5 in I. Assumption (H3) implies $\mathbf{A} 3$ of I (the class of Fermi surfaces considered there was much bigger than that of positive curvature). Thus all theorems of I apply to this situation.

Assumption (H4) is a technical assumption that ensures that the locations of the singularities of certain Jacobians depend continuously on the external momenta. The specific number $\frac{1}{8}$ in (2.10) is certainly not optimal.

Assumption (H4') is needed in the $d=2$ asymmetric case (where typically $\mathbf{a}(\mathbf{p}) \neq-\mathbf{p}$ ). That the curvatures differ at almost every $\mathbf{p} \in S$ and its antipode is needed in a volume bound for the particleparticle bubble (see Appendix C); this is the bound that also implies that the Cooper channel is turned off in the asymmetric case.

Assumption (H5) restricts the density $n$ to be small enough, to avoid certain umklapp processes. E.g. in the Hubbard model, where $e(\mathbf{p})=-2\left(\cos p_{1}+\cos p_{2}\right)-\mu$, this is fulfilled for densities $n<0.369$ (here $n=1$ is half-filling). We shall need Assumption (H5) only to prove statements about the second-order graph and the RPA graphs. In particular, the optimal volume improvement bound (and the bounds for values of large graphs in III do not require (H5)). Thus a detailed investigation of the role of (H5) can be done essentially in second order. The explicit role of (H5) in our proofs will be discussed in Section 3.3. 


\subsection{Some elementary consequences}

(H2) and (H3) imply that there are $\varepsilon_{1}>0$ and $g_{\mathrm{o}}>0$ such that

$$
\forall \mathbf{p} \in U_{\varepsilon_{1}}(S): \quad|\nabla e(\mathbf{p})| \geq g_{\mathrm{o}}
$$

The filling restriction (H5) has the following consequence.

Lemma 2.1 Let $S$ fulfil (H3) and (H5). Then

(i) If $\mathbf{p}, \mathbf{q}, \mathbf{r} \in S$ and $2 \mathbf{q}=\mathbf{p}+\mathbf{r}$, then $\mathbf{p}=\mathbf{q}=\mathbf{r}$

(ii) For a given $\mathbf{p}_{2} \in S$, the equation

$$
\mathbf{p}_{1}-\mathbf{p}=\mathbf{a}\left(\mathbf{p}_{2}\right)-\mathbf{p}_{2}, \quad \mathbf{p}_{1}, \mathbf{p} \in S
$$

has only the solution $\mathbf{p}_{1}=\mathbf{a}\left(\mathbf{p}_{2}\right), \mathbf{p}=\mathbf{p}_{2}$.

Proof: $\quad$ By (H5), both sides of the equations in $(i)$ and $(i i)$ are vectors in $\stackrel{\mathcal{F}}{ }$, which is an open convex subset of $\mathbb{R}^{d}$. Therefore, to determine their solutions, we may consider $S$ as a subset of $\mathbb{R}^{d}$ instead of $\mathcal{B}$. ( $i$ ) If $2 \mathbf{q}=\mathbf{p}+\mathbf{r}$ then the three points $\mathbf{q}, \mathbf{r}=2 \mathbf{q}-\mathbf{p}=\mathbf{q}+(\mathbf{q}-\mathbf{p})$ and $\mathbf{p}=\mathbf{q}-(\mathbf{q}-\mathbf{p})$ are collinear. But $\mathbf{p}, \mathbf{q}, \mathbf{r} \in S$, so by strict convexity collinearity can hold only if $\mathbf{p}=\mathbf{q}=\mathbf{r}$. (ii) It is obvious that $\mathbf{p}_{\mathbf{1}}=\mathbf{a}\left(\mathbf{p}_{2}\right)$, $\mathbf{p}=\mathbf{p}_{2}$ is a solution of (2.12). We only have to show that there is no other solution. Let $\mathbf{r}=\frac{\mathbf{p}_{\mathbf{2}}-\mathbf{a}\left(\mathbf{p}_{\mathbf{2}}\right)}{\left|\mathbf{p}_{\mathbf{2}}-\mathbf{a}\left(\mathbf{p}_{\mathbf{2}}\right)\right|}$. We show uniqueness by proving that the chord from $\mathbf{p}_{2}$ to its antipode $\mathbf{a}\left(\mathbf{p}_{2}\right)$ is strictly longer than any other chord of $S$ in direction $\mathbf{r}$. This is sufficient because for $\mathbf{p}$ and $\mathbf{p}_{\mathbf{1}}$ to be a solution of (2.12), the chord given by $\mathbf{p}_{1}-\mathbf{p}$ must point in direction $\mathbf{r}$, i.e. $\mathbf{p}_{1}-\mathbf{p}=\left|\mathbf{p}_{2}-\mathbf{a}\left(\mathbf{p}_{2}\right)\right| \mathbf{r}$.

Consider the family of lines $L_{\mathbf{p}}=\{\mathbf{p}-t \mathbf{r}: t \in \mathbb{R}\}$, parametrized by $\mathbf{p} \in S$. As $\mathbf{p}$ is varied, the line $L_{\mathbf{p}}$ slides over $S$, and thus defines a chord in direction $\mathbf{r}$. All chords in direction $\mathbf{r}$ are produced by an $L_{\mathbf{p}}$ for some $\mathbf{p}$. Consider the tangent planes to $S$ at $\mathbf{p}_{2}$ and $\mathbf{a}\left(\mathbf{p}_{2}\right)$. They are parallel. Hence any line segment parallel to $\mathbf{r}$ whose endpoints lie strictly between the two tangent planes has length strictly less than $\left|\mathbf{p}_{2}-\mathbf{a}\left(\mathbf{p}_{2}\right)\right|$. Because $S$ is strictly convex, it remains on one side of each of the tangent planes, and it intersects the tangent planes only at $\mathbf{p}_{2}$ and $\mathbf{a}\left(\mathbf{p}_{2}\right)$. Thus every other chord in direction $\mathbf{r}$ is shorter than that from $\mathbf{p}_{2}$ to $\mathbf{a}\left(\mathbf{p}_{2}\right)$.

\section{Radial and angular coordinates}

For $r_{\mathrm{o}}>0$, let $\mathcal{T}=\left(-2 r_{\mathrm{o}}, 2 r_{\mathrm{o}}\right) \times S$. Then there is an $r_{\mathrm{o}}>0$ and a $C^{k}$-diffeomorphism $\phi: \mathcal{T} \rightarrow \phi(\mathcal{T})=$ $\mathcal{U}(S) \subset \mathcal{B},(\rho, \sigma) \mapsto \mathbf{p}=\phi(\rho, \sigma)$ such that $e(\phi(\rho, \sigma))=\rho$, and such that

$$
|\nabla e(\phi(\rho, \sigma))| \geq g_{\mathrm{o}}>0
$$

for all $|\rho|<r_{\mathrm{o}}$ and all $\sigma . \phi$ is constructed explicitly in Lemma 2.1 in I, using the integral curves of a $C^{\infty}$ vector field $u$ that is transversal to $S$ in the sense that

$$
u(\mathbf{p}) \cdot \nabla e(\mathbf{p}) \geq u_{\mathrm{o}} \geq \frac{g_{\mathrm{o}}}{2} .
$$


We shall assume that $\phi$ is given by this specific construction and use its properties, and therefore ask you to recall it from I. For ease of notation, we shall also write $\mathbf{p}(\rho, \sigma)$ for $\phi(\rho, \sigma)$, and $\mathbf{p}(\sigma)$ for $\mathbf{p}(0, \sigma)$. Since $\mathbf{p}$ is $C^{k}$ in $\rho$ and $\sigma$, the Jacobian

$$
J(\rho, \sigma)=\left|\operatorname{det} \mathbf{p}^{\prime}(\rho, \sigma)\right|
$$

of the corresponding change of variables $\mathbf{p} \rightarrow(\rho, \sigma)$ is $C^{k-1}$.

We also denote $S_{\rho}=\{\mathbf{p} \in \mathcal{B}: e(\mathbf{p})=\rho\}$. For $\rho$ small enough, $S_{\rho}$ is also strictly convex, and we denote the antipodal map on $S_{\rho}$ by $\mathbf{a}_{\rho}$. It is defined for $\mathbf{p} \in S_{\rho}$ by

$$
\mathbf{n}\left(\mathbf{a}_{\rho}(\mathbf{p})\right)=-\mathbf{n}(\mathbf{p}) \quad \text { and } \quad \mathbf{a}_{\rho}(\mathbf{p}) \in S_{\rho}
$$

We assume that $r_{\mathrm{o}}$ is chosen such that strict convexity of $S_{\rho}$ holds for all $|\rho| \leq r_{\mathrm{o}}$.

$r_{\mathrm{o}}$ will be chosen smaller in what follows, it depends, however, only on $|e|_{2}$ and the geometry of the Fermi surface, i.e., on $g_{\mathrm{o}}$ and the constant $w_{\mathrm{o}}$ defined in this section (which is related to the minimal curvature of $S$ ). The antipodal map $\mathbf{a}_{\rho}$ induces a map $a_{\rho}(\sigma)$ by

$$
\mathbf{p}\left(0, a_{\rho}(\sigma)\right)=\mathbf{a}_{\rho}(\mathbf{p}(0, \sigma))
$$

For $\rho=0$, we denote $a(\sigma)=a_{\mathrm{o}}(\sigma)$.

There is a $C^{k}$-diffeomorphism that maps $S$ to the unit sphere $S^{d-1}, \sigma \mapsto \theta$. We use this variable $\theta$ in the following and keep the same notation for the map $(\rho, \theta) \rightarrow \mathbf{p}(\rho, \theta)$, the Jacobian $J$ and the antipode $a$. The diffeomorphism from $S$ to $S^{d-1}$ depends on $e$, but it is a $C^{k}$-diffeomorphism because $S$ is a $C^{k}$ surface, and the Jacobian and its derivatives are bounded uniformly for all $e$ satisfying $(\mathrm{H} 2)_{k, 0}$ and (H3).

\section{Two Dimensions}

For $d=2$, the variable $\theta \in S^{1}$ is simply an angular variable. The map $\phi$ is $(\rho, \theta) \mapsto \mathbf{p}(\rho, \theta)$. It is $C^{k}$ in both variables. The vector $\partial_{\theta} \mathbf{p}$ is nonzero at all points in $\mathcal{T}$ because $\phi$ is a diffeomorphism. By definition of the coordinates $\rho$ and $\theta$ (see Lemma 2.1 in I), the variable $\theta$ is constant on the integral curves of $u$, which are fixed independently of $\rho$. Therefore, if we now regard $\theta$ as a variable in $\mathbb{R}$, the period of the map $\theta \mapsto \mathbf{p}(\rho, \theta)$ is the same for all $\rho$ with $|\rho|<r_{\mathrm{o}}$.

Define the matrix $e^{\prime \prime}(\mathbf{p})$ by

$$
\left(e^{\prime \prime}(\mathbf{p})\right)_{i j}=\frac{\partial^{2} e}{\partial p_{i} \partial p_{j}}(\mathbf{p})
$$

let $(a, b)=\sum_{i} a_{i} b_{i}$ and let

$$
w(\mathbf{p})=\left(\partial_{\theta} \mathbf{p}, e^{\prime \prime}(\mathbf{p}) \partial_{\theta} \mathbf{p}\right) .
$$

At fixed $\rho, \mathbf{p}(\rho, \theta)$ is a parametrization of the curve $S_{\rho}$. Denote the unit tangent by

$$
\mathbf{t}(\mathbf{p})=\frac{\partial_{\theta} \mathbf{p}}{\left|\partial_{\theta} \mathbf{p}\right|}
$$

and define the arclength as $s(\rho, \theta)=\int_{0}^{\theta} d \vartheta\left|\partial_{\theta} \mathbf{p}(\rho, \vartheta)\right|$. Then

$$
\partial_{\theta}^{2} \mathbf{p}(\rho, \theta)=\frac{\partial^{2} s}{\partial \theta^{2}} \mathbf{t}(\mathbf{p}(\rho, \theta)) \pm \kappa(\rho, \theta)\left(\frac{\partial s}{\partial \theta}\right)^{2} \mathbf{n}(\mathbf{p}(\rho, \theta)) .
$$


The sign is -1 if $e(\mathbf{p})<0$ inside $S$ and +1 otherwise.

Lemma 2.2 By (H2), for all $|\rho|<r_{\mathrm{o}}$ and all $\theta$,

$$
\kappa(\rho, \theta)|\nabla e(\mathbf{p}(\rho, \theta))|=-\frac{w(\mathbf{p}(\rho, \theta))}{\left|\partial_{\theta} \mathbf{p}(\rho, \theta)\right|^{2}}
$$

By (H3), there is $\kappa_{\mathrm{o}}>0$ such that $\kappa(0, \theta) \geq 2 \kappa_{\mathrm{o}}>0$ for all $\theta$. Thus $r_{\mathrm{o}}>0$ can be chosen so small that for all $|\rho|<r_{\mathrm{o}}$ and all $\theta, \kappa(\rho, \theta) \geq \kappa_{\mathrm{o}}$, and there is $w_{\text {min }}>0$ such that for all $|\rho|<r_{\mathrm{o}}$ and all $\theta$

$$
|w(\mathbf{p}(\rho, \theta))| \geq w_{\min }\left|\partial_{\theta} \mathbf{p}(\rho, \theta)\right|^{2} .
$$

Moreover, $\left|\partial_{\theta}^{2} \mathbf{p}(\rho, \theta)\right| \geq \frac{w_{\min }}{|e|_{1}}\left|\partial_{\theta} \mathbf{p}(\rho, \theta)\right|^{2}$.

Proof: $\quad e(\mathbf{p}(\rho, \theta))=\rho$ for all $\theta$, so

$$
\begin{aligned}
\nabla e(\mathbf{p}(\rho, \theta)) \cdot \partial_{\theta} \mathbf{p}(\rho, \theta) & =0 \\
\text { and } \quad w(\mathbf{p}(\rho, \theta))+\nabla e(\mathbf{p}(\rho, \theta)) \cdot \partial_{\theta}^{2} \mathbf{p}(\rho, \theta) & =0 .
\end{aligned}
$$

We insert (2.21) and use $\mathbf{t} \cdot \mathbf{n}=0$ and $\frac{\partial s}{\partial \theta}=\left|\partial_{\theta} \mathbf{p}\right|$, to get

$$
w(\mathbf{p}(\rho, \theta)) \pm \kappa(\rho, \theta)\left|\partial_{\theta} \mathbf{p}\right|^{2}|\nabla e(\mathbf{p}(\rho, \theta))|=0
$$

Thus $|w(\mathbf{p}(\rho, \theta))| \geq w_{\text {min }}\left|\partial_{\theta} \mathbf{p}(\rho, \theta)\right|^{2}$ holds with

$$
w_{\min }=\kappa_{\mathrm{o}} g_{\mathrm{o}}
$$

and, by (2.24),

$$
|e|_{1}\left|\partial_{\theta}^{2} \mathbf{p}(\rho, \theta)\right| \geq \mid \nabla e\left(\left.\mathbf{p}(\rho, \theta)|| \partial_{\theta}^{2} \mathbf{p}(\rho, \theta) \cdot \mathbf{n}(\mathbf{p}(\rho, \theta))\left|\geq w_{\min }\right| \partial_{\theta} \mathbf{p}(\rho, \theta)\right|^{2}\right.
$$

We transform to a new angular variable, which is a multiple of the arclength of the curve $\mathbf{p}(0, \theta)$, so that in the new variables, $\partial_{\theta} \mathbf{p}$ is of constant length for $\rho=0$, and normalized such that the period is $2 \pi$. We give the argument in detail to show that no differentiability is lost by this change of variables. Let $\left|\partial_{\theta} \mathbf{p}(\rho, \theta)\right|=v(\rho, \theta)$. Since $v(\rho, \theta)>0$ for all $|\rho| \leq r_{\mathrm{o}}$ and all $\theta$, we can define a new variable $\tilde{\theta}$ as follows. Let $\theta_{\mathrm{o}}$ be fixed and $P>0$. Define

$$
\tilde{\theta}(\theta)=P \int_{\theta_{\mathrm{o}}}^{\theta} d \vartheta v(0, \vartheta)
$$

$v(0, \theta)$ is $C^{k-1}$, so the map $\theta \mapsto \tilde{\theta}$ is $C^{k}$. It is a diffeomorphism because $v(0, \theta)>0$, and $\tilde{\theta}\left(\theta_{0}\right)=0$. Denoting its inverse by $\theta(\tilde{\theta})$ and $\tilde{\mathbf{p}}(\rho, \tilde{\theta})=\mathbf{p}(\rho, \theta(\tilde{\theta}))$, we have

$$
\frac{\partial}{\partial \tilde{\theta}} \tilde{\mathbf{p}}(\rho, \tilde{\theta})=\frac{\partial \theta}{\partial \tilde{\theta}} \partial_{\theta} \mathbf{p}(\rho, \theta(\tilde{\theta}))=\frac{1}{P v(0, \theta)} \partial_{\theta} \mathbf{p}(\rho, \theta)
$$




$$
\left|\partial_{\tilde{\theta}} \tilde{\mathbf{p}}(0, \tilde{\theta})\right|=\frac{1}{P} \quad \text { for all } \tilde{\theta}
$$

Unless stated otherwise, we take the variable $\tilde{\theta}$ and choose $P$ such that the period is $2 \pi$, i.e. $\tilde{\theta} \in \mathbb{R} / 2 \pi \mathbb{Z}$. We also drop the tilde, so that in summary

$$
\theta \in \mathbb{R} / 2 \pi \mathbb{Z}, \quad\left|\partial_{\theta} \mathbf{p}(0, \theta)\right|=\frac{1}{P}
$$

Calling

$$
w_{\mathrm{o}}=\frac{w_{\min }}{P^{2}}=\frac{\kappa_{\mathrm{o}} g_{\mathrm{o}}}{P^{2}},
$$

the statement of Lemma 2.2 is: for all $|\rho|<r_{\mathrm{o}}$ and all $\theta$,

$$
|w(\mathbf{p})| \geq w_{\mathrm{o}}
$$

In terms of the new coordinate $\theta$, the antipodal map $a$ on the Fermi surface satisfies

$$
\partial_{\theta} \mathbf{p}(0, a(\theta))=-\partial_{\theta} \mathbf{p}(0, \theta)
$$

$a$ is $C^{k-1}$ in $\theta$. If (Sy) holds, $a$ is $C^{\infty}$ because $a(\theta)=\theta+\pi$ for all $\theta$. If $e$ is asymmetric, (H4) implies that for all $\theta$,

$$
\frac{7}{8} \leq \frac{\partial a}{\partial \theta} \leq \frac{9}{8}
$$

In the symmetric case this is trivially true because $a(\theta)=\theta+\pi$. By (2.34),

$$
\partial_{\theta}^{2} \mathbf{p}(0, a(\theta)) \frac{\partial a(\theta)}{\partial \theta}=-\partial_{\theta}^{2} \mathbf{p}(0, \theta)
$$

By choice of the coordinate $\theta, \partial_{\theta}^{2} \mathbf{p}(0, \theta) \propto \mathbf{n}(\mathbf{p}(0, \theta))$, so

$$
\frac{\partial a}{\partial \theta}=\frac{\left|\partial_{\theta}^{2} \mathbf{p}(0, \theta)\right|}{\left|\partial_{\theta}^{2} \mathbf{p}(0, a(\theta))\right|}=\frac{\kappa(0, \theta)}{\kappa(0, a(\theta))} .
$$

Thus (H4) is simply a condition on the ratio of the curvatures at $\mathbf{p}$ and its antipode.

\section{The assumption (H4')}

For $d=2$, and $e$ not obeying (Sy), we impose

(H4') The function $\frac{\partial a}{\partial \theta}$ obeys $\frac{\partial a}{\partial \theta}=1$ only at finitely many points $\theta^{(1)}, \ldots, \theta^{(N)}$. There is $\delta_{\mathrm{o}}>0$ such that for all $k, l \in\{1, \ldots, N\}:$ if $k \neq l, U_{2 \delta_{\mathrm{o}}}\left(\theta^{(k)}\right) \cap U_{2 \delta_{\mathrm{o}}}\left(\theta^{(l)}\right)=\emptyset, \frac{\partial a}{\partial \theta}$ is monotonic on $U_{2 \delta_{\mathrm{o}}}\left(\theta^{(k)}\right)$, and there is a constant $K_{a}>0$ such that for all $k \in\{1, \ldots, N\}$ and all $\theta, \theta^{\prime} \in U_{\delta_{\mathrm{o}}}\left(\theta^{(k)}\right)$,

$$
\left|\frac{\partial a}{\partial \theta}(\theta)-\frac{\partial a}{\partial \theta}\left(\theta^{\prime}\right)\right| \geq K_{a}\left|\theta-\theta^{\prime}\right|
$$

It should be clear that for any asymmetric surface, points $\theta^{(1)}, \ldots, \theta^{(N)}$, where $\frac{\partial a}{\partial \theta}=1$, must exist: $\frac{\partial a}{\partial \theta}$ is a $2 \pi$-periodic continuous function that satisfies

$$
\frac{\partial a}{\partial \theta}(a(0))=\frac{\kappa(0)}{\kappa(a(0))}=\left(\frac{\kappa(0)}{\kappa(a(0))}\right)^{-1}=\left(\frac{\partial a}{\partial \theta}(0)\right)^{-1}
$$


by (2.37). By the intermediate value theorem, it must either be constant (then $e$ is symmetric), or it must take the value 1 at least twice. If we suppose for a moment that $\frac{\partial a}{\partial \theta}$ is differentiable, a similar argument shows that $\frac{\partial^{2} a}{\partial \theta^{2}}$ must also have zeros. (H4') states (without referring to such additional derivatives), that at those $\theta$ where the curvature ratio is one, this ratio varies at least linearly. The curvature ratio turns up, and hence (H4') will be used, only in the proof of Theorem $1.3(\mathrm{ii})$, more specifically in the one-loop volume estimate that shows boundedness of the particle-particle ladder if ( $\mathrm{Sy}$ ) does not hold (if (H4') holds instead).

\section{Higher Dimensions}

Let $\partial^{2} e$ denote the $d \geq 3$ analogue of the matrix defined in (2.18). By (H4), there is, for each $\theta \in S^{d-1}$, a basis $\mathbf{v}_{1}, \cdots, \mathbf{v}_{d-1}$ for the orthogonal complement to $\nabla e(\mathbf{p}(\theta))$ such that the matrices

$$
\begin{aligned}
& C_{\theta}=\left[\left(\mathbf{v}_{i}, \partial^{2} e(\mathbf{p}(0, \theta)) \mathbf{v}_{j}\right)\right]_{1 \leq i, j \leq d-1} \\
& A_{\theta}=\left[\left(\mathbf{v}_{i}, \partial^{2} e(\mathbf{p}(0, a(\theta))) \mathbf{v}_{j}\right)\right]_{1 \leq i, j \leq d-1}
\end{aligned}
$$

obey

$$
\left|\frac{|\nabla e(\mathbf{p}(\theta))|}{|\nabla e(\mathbf{p}(a(\theta)))|} A_{\theta} C_{\theta}^{-1}-v \mathbb{1}\right| \leq 1 / 5
$$

for some $v \in\{ \pm 1\}$ (in the symmetric case, $C_{\theta}=A_{\theta}$ and $|\nabla e(\mathbf{p}(\theta))|=|\nabla e(\mathbf{p}(a(\theta)))|$ ). The matrix $C_{\theta}$ is invertible because $S$ has positive curvature everywhere.

\subsection{Covariance}

The propagator for the independent $(\lambda=0)$ electrons is

$$
\left(G_{\mathrm{o}}\right)_{\alpha \alpha^{\prime}}(p)=\delta_{\alpha \alpha^{\prime}} C\left(p_{\mathrm{o}}, e(\mathbf{p})\right)
$$

with

$$
C(\omega, E)=\frac{e^{i \omega 0^{+}}}{i \omega-E}
$$

This notation means the usual boundary value for distributions, e.g.

$$
\int d^{d+1} p \frac{e^{i p_{\mathrm{o}} 0^{+}}}{i p_{\mathrm{o}}-e(\mathbf{p})}=\lim _{\tau \downarrow 0} \int d^{d+1} p \frac{e^{i p_{\mathrm{o}} \tau}}{i p_{\mathrm{o}}-e(\mathbf{p})} .
$$

The reason for this limiting prescription is that by Fourier transformation, at temperature $T=\frac{1}{\beta}$, the Fermi distribution function, which determines the thermodynamics of independent electrons, is given by

$$
\frac{1}{1+e^{\beta E}}=\lim _{\tau \downarrow 0} \frac{1}{\beta} \sum_{n \in \mathbb{Z}} \frac{e^{i \omega_{n} \tau}}{i \omega_{n}-E}
$$

where $\omega_{n}=(2 n+1) \pi T$. In the limit $T \rightarrow 0$, the frequency sum becomes an integral.

The behaviour of perturbation theory is determined by the properties of $C$ and the interaction potential $\hat{v}$. It is obvious that $\int_{1}^{\infty}|C(p)| d p_{\mathrm{o}}$ is infinite so that one must not take the absolute values inside loop integrals 
containing only one propagator. As will be shown, this is harmless, however, and the mathematically difficult, and physically relevant, singularity is in the infrared, at $p_{\mathrm{o}}=0$ and $e(\mathbf{p})=0$. This singularity implies that

$$
\int_{p_{0}^{2}+E^{2} \leq 1}\left|C\left(p_{0}, E\right)\right|^{n}=\infty
$$

for all $n \geq 2$, which causes the divergences of unrenormalized perturbation theory [FT1,FT2,FST]. The singularity of $C$ at $p_{\mathrm{o}}^{2}+E^{2}=0$ is the important one because it determines the long-distance behaviour of the fermion two-point function.

\section{The scale zero effective action}

To separate the harmless, short-distance part of the propagator from the long-distance part, we first integrate over all fields whose momenta are not in a neighbourhood of the Fermi surface. This includes in particular the fields with large $\left|p_{\mathrm{o}}\right|$. This integration produces an effective interaction, which we call the scale zero effective action, for the remaining fields, which are then subject to a fixed ultraviolet cutoff.

To integrate over the fields with momenta away from the Fermi surface, we split the propagator $C$ into a scale zero part $C_{\mathrm{o}}$ where $p_{\mathrm{o}}^{2}+E^{2} \geq$ const $>0$ and an infrared part $C_{<0}$ where $p_{\mathrm{o}}^{2}+E^{2}$ can get arbitrarily close to zero, as follows. Let $M \geq \max \left\{4^{3}, \frac{1}{r_{\mathrm{o}}}\right\}$ (then $|e(\mathbf{p})|<M^{-1}$ implies $|\rho|<r_{\mathrm{o}}$ ), and let $a \in C^{\infty}\left(\mathbb{R}_{\mathrm{o}}^{+},[0,1]\right)$ be such that $a(x)=0$ for $0 \leq x \leq M^{-4}, a(x)=1$ for $x \geq M^{-2}$, and $a^{\prime}(x)>0$ for all $x \in\left(M^{-4}, M^{-2}\right)$. Define

$$
\begin{aligned}
C_{\mathrm{o}}\left(p_{\mathrm{o}}, E\right) & =a\left(p_{\mathrm{o}}^{2}+E^{2}\right) C\left(p_{\mathrm{o}}, E\right) \\
C_{<0}\left(p_{\mathrm{o}}, E\right) & =\left(1-a\left(p_{\mathrm{o}}^{2}+E^{2}\right)\right) C\left(p_{\mathrm{o}}, E\right)
\end{aligned}
$$

Since $C=C_{\mathrm{o}}+C_{<0}$, the effective action

$$
e^{\mathcal{G}(\chi, \bar{\chi})}=\int d \mu_{C}(\psi, \bar{\psi}) e^{\lambda V^{(0)}(\psi+\chi, \bar{\psi}+\bar{\chi})}
$$

can be written as

$$
e^{\mathcal{G}(\chi, \bar{\chi})}=\int d \mu_{C_{<0}}(\psi, \bar{\psi}) e^{\mathcal{V}_{\mathrm{eff}}^{(0)}(\lambda, \psi+\chi, \bar{\psi}+\bar{\chi})}
$$

where

$$
e^{\mathcal{V}_{\mathrm{eff}}^{(0)}(\lambda, \chi, \bar{\chi})}=\int d \mu_{C_{\mathrm{o}}}(\psi, \bar{\psi}) e^{\lambda V^{(0)}(\psi+\chi, \bar{\psi}+\bar{\chi})} .
$$

$\mathcal{V}_{\mathrm{eff}}^{(0)}(\lambda, \psi, \bar{\psi})$ is a formal power series in $\lambda$ :

$$
\mathcal{V}_{\mathrm{eff}}^{(0)}(\lambda, \psi, \bar{\psi})=\sum_{r=1}^{\infty} \lambda^{r} \sum_{m=0}^{\bar{m}(r)} \int d p_{1} \ldots d p_{2 m} \delta\left(\sum_{i=1}^{m}\left(p_{i}-p_{m+i}\right)\right) V_{m, r}^{(0)}\left(p_{1}, \ldots, p_{2 m}\right) \prod_{i=1}^{m} \bar{\psi}\left(p_{i}\right) \psi\left(p_{m+i}\right)
$$

The propagator that appears in $\mathcal{V}_{\text {eff }}$ is $C_{0}$, which has no infrared singularity.

Lemma 2.3 Assume $(\mathrm{H} 1)_{k, h}$ and $(\mathrm{H} 2)_{k, h}$, with $k \geq 2$ and $h \geq 0$. The $V_{m, r}^{(0)}$ are all bounded and $C^{k, h}$ in the external momenta, i.e.

$$
\left|\left(V^{(0)}\right)_{m, r}\right|_{k, h} \leq K(m)^{r} r !
$$


Proof: See Appendix D.

Remark 2.4 In fact, the scale zero effective action is analytic in $\lambda$ (so the $r$ ! is not really there on the right side of (2.52)). Since $C_{\mathrm{o}}$ has no singularity, one can show that $\mathcal{U}_{v}$ is analytic in $\lambda$ in a disk independent of the volume and the temperature, by adapting the determinant bound given in [FMRT] suitably. Recall that momentum space is $\mathbb{R} \times \mathcal{B}$ with $\mathcal{B}=\mathbb{R}^{d} / \Gamma^{\#}$ compact. So the spatial components of momenta are subject to a fixed ultraviolet cutoff. Consequently, there is no 'stability of matter' problem.

The term $\left(V^{(0)}\right)_{1, r}$ is a bilinear term in the fermions. Since it is $C^{k}$ and $O(\lambda)$, and since $\lambda$ is small, it does not change the properties of $e$. We simply absorb it into $e$ without changing notation.

Thus the only difference between the original model and the one with interaction $V_{\mathrm{eff}}^{(0)}$ is that the latter contains vertices with $2 m \geq 6$ external legs, whose vertex functions are at least of order $\lambda^{2}$, and which are $C^{k, h}$ in the external momenta with bounds that are scale independent. This is a minor complication which is easily taken into account (it will mainly concern us in III).

\section{The scale decomposition in the infrared}

We now turn to the essential part of the problem. We decompose the infrared part $C_{<0}$ of the propagator into slices as in I: Let $f(x)=a(x)-a\left(x / M^{2}\right)$, then $1-a(x)=\sum_{j<0} f\left(M^{-2 j} x\right)$, and (omitting the $e^{i p_{\mathrm{o}} 0^{+}}$ since the limits can be taken inside the integrals because large $p_{\mathrm{o}}$ do not occur in the infrared part of the propagator)

$$
\begin{aligned}
C_{<0}\left(p_{\mathrm{o}}, E\right) & =\frac{1-a\left(p_{\mathrm{o}}^{2}+E^{2}\right)}{i p_{\mathrm{o}}-E}=\sum_{j<0} C_{j}\left(p_{\mathrm{o}}, E\right) \\
C_{j}\left(p_{\mathrm{o}}, E\right) & =\frac{f\left(M^{-2 j}\left(p_{\mathrm{o}}^{2}+E^{2}\right)\right)}{i p_{\mathrm{o}}-E} .
\end{aligned}
$$

The point of this decomposition is that the propagator on scale $j, C_{j}$, has simple properties: it is easy to prove (see I, Lemmas 2.1 and 2.3) that

$$
\max _{|\alpha|=s}\left|D^{\alpha} C_{j}\left(p_{\mathrm{o}}, e(\mathbf{p})\right)\right| \leq W_{s} M^{-(s+1) j} \mathbb{1}\left(\left|i p_{\mathrm{o}}-e(\mathbf{p})\right| \leq M^{j}\right)
$$

where $D^{\alpha}$ is a derivative with respect to $p$ ( $\alpha$ is a multiindex with $|\alpha|=s, 0 \leq s \leq k$ ) of order $s$. The indicator functions take the value $\mathbb{1}(X)=1$ if $X$ is true and $\mathbb{1}(X)=0$ otherwise. In words: on 'slice' number $j$, the propagator is for all $\left(p_{\mathrm{o}}, \mathbf{p}\right)$ of absolute value at most $M^{2-j},\left(s=0\right.$ in $\left.(2.54) ; W_{\mathrm{o}}=M^{2}\right)$, and every derivative produces another large factor $M^{-j}$. The constant $W_{s}$ depends on $|e|_{s}$ and on $g_{\mathrm{o}}$. Moreover, the support of $C_{j}$ is contained in the product of an interval of length $2 M^{j}$ in $p_{\mathrm{o}}$ and a thin shell of thickness const $M^{j}$ around the Fermi surface $S$. By our choice of $M, M^{-1}<r_{\mathrm{o}}$, so for all $j<0$ this shell is contained in the region where the variables $\rho$ and $\theta$ can be used.

In infinite volume, we introduce an infrared cutoff by restricting the sum in (2.53) to $j \geq I$, where $I \in \mathbb{Z}, I<0$ (see I for details). In infinite volume the limit $I \rightarrow-\infty$ is the definition of the model. We 
shall show in another paper that the so defined infinite volume and zero temperature Green functions are perturbatively identical to those obtained by taking a finite volume, positive temperature model and letting the volume tend to infinity and afterwards the temperature go to zero.

The heart of the analysis of this paper concerns regularity properties of the self-energy in the spatial part $\mathbf{p}$ of the momentum. It will be obvious from studying the proofs that this analysis works uniformly for positive temperature as well as for temperature zero.

As explained, $\int d p_{\mathrm{o}} d E\left|C_{<0}\left(p_{\mathrm{o}}, E\right)\right|<\infty$, but $\int d p_{\mathrm{o}} d E\left|C_{<0}\left(p_{\mathrm{o}}, E\right)\right|^{2}=\infty$ because of the singularity at $p_{\mathrm{o}}=E=0$ (see I, Section 1.4). To get finite perturbative Green functions, we renormalize. To this end, we use the projection $\mathbf{P}$ introduced in Section 2.2 of I, i.e.

$$
\mathbf{P}(\mathbf{p}(\rho, \sigma))=\mathbf{p}(0, \sigma),
$$

and for continuous functions $F(p)$,

$$
(\ell F)\left(p_{\mathrm{o}}, \mathbf{p}(\rho, \sigma)\right)= \begin{cases}F(0, \mathbf{p}(0, \sigma)) \chi(\rho) & |\rho|<2 r_{\mathrm{o}} \\ 0 & \text { otherwise }\end{cases}
$$

where $\chi \in C^{\infty}(\mathbb{R}, \mathbb{R}), \chi(x)=1$ for $|x|<r_{\mathrm{o}}$ and $\chi(x)=0$ for $|x|>2 r_{\mathrm{o}}$, and $\chi$ decreases in $|x|$. The counterterm and the self-energy are then defined recursively in $r$, the order in $\lambda$, as follows. In first order, let $\Sigma_{1}^{u n s u b}(p)$ be the sum of the values of the first order 1PI two-legged graphs, evaluated according to the Feynman rules of the model. Then $K_{1}(\mathbf{p})=\left(\ell \Sigma_{1}^{u n s u b}\right)(p)=\Sigma_{1}^{u n s u b}(0, \mathbf{P}(\mathbf{p}))$, and $\Sigma_{1}(p)=\Sigma_{1}^{u n s u b}(p)-K_{1}(\mathbf{p})$. Assuming that $\Sigma_{r}$ and $K_{r}$ have been defined, $\Sigma_{r+1}^{u n s u b}$ is the sum of values of all 1PI two-legged graphs with all two-legged insertions 1PI $T(p)$ being of the form $\Sigma_{s}, s<r$, i.e. subtracted on the Fermi surface $\left(T(p)-T(0, \mathbf{P}(\mathbf{p}))\right.$ appears). Then $K_{r+1}=\ell \Sigma_{r+1}$ and $\Sigma_{r+1}=\Sigma_{r+1}^{u n s u b}-K_{r+1}$. Note that $K_{r}^{I}$ actually only depends on $\mathbf{P}(\mathbf{p}) \in S$; this will be very important in the following. In the scale decomposition, with an infrared cutoff $I, K_{r}^{I}$ appears as a sum over trees and compatible labelled graphs (for details, not needed here, see I, (2.76)). This scale decomposition is a way of dealing with the functions that allows us to do estimates that are even hard to get in the few cases where one can use exact calculations. It is proven in I, Theorem 1.2, that the sequence of functions $K_{r}^{I}$ and their derivatives with respect to $\mathbf{p}$ converge uniformly as $I \rightarrow-\infty$ and that

$$
K_{r}=\lim _{I \rightarrow-\infty} K_{r}^{I}
$$

is $C^{1}$ in $\mathbf{p}$. In the next chapter, we show that $K_{2}$ is $C^{2}$ if $e$ and $\hat{V}$ are $C^{2}$.

\section{Regularity in Second Order}

In this chapter, we give a detailed explanation of the problem and its solution for the example of the secondorder counterterm $K^{(2)}$ (the problem begins to be nontrivial in second order). After motivating the problem, we prove Theorem 1.2. We also explain the basics of the scale decomposition when we deal with the second order graph. 

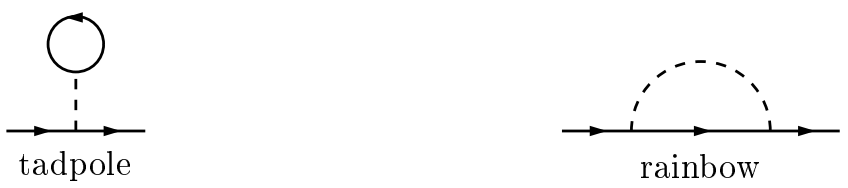

Figure 1

\subsection{Preparations}

The graphs that contribute to first order are shown in Figure 1.

They have the values

$$
Y_{1}(q)=\int_{\mathbb{R} \times \mathcal{B}} d p P(p, q) C\left(p_{\mathrm{o}}, e(\mathbf{p})\right)
$$

with

$$
P(p, q)= \begin{cases}\langle q p|V| p q\rangle & \text { rainbow } \\ \langle q p|V| q p\rangle & \text { tadpole. }\end{cases}
$$

(we use matrix notation for the spin sums; the tadpole involves a spin trace which we omitted in the notation because it is inessential for the regularity problem. Also, $\left.d p=\frac{d^{d+1} p}{(2 \pi)^{d+1}}\right)$. Since the only factor that depends on $q$ is the interaction function, and since $C$ is integrable, it is trivial to take the limit $I \rightarrow-\infty$ of $Y_{1}$, and it is $C^{k, h}$ in the external momentum by $(\mathrm{H} 1)_{k, h}$. Graphically speaking, the external momentum either enters no line of the graph at all (for the tadpole term), or it can be routed through the interaction line (for the rainbow term).

The second order gets contributions from the graphs shown in Figure 2. (recall that only 1PI two-legged diagrams contribute to the self-energy and the counterterm).

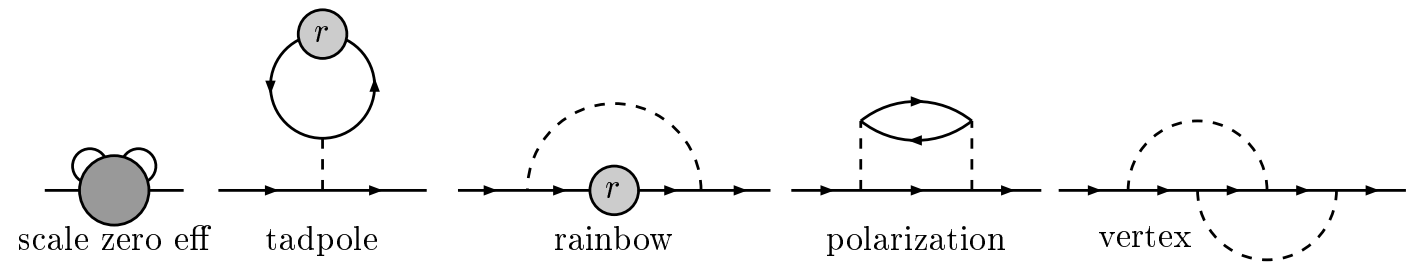

Figure 2

The shaded disk in the second order rainbow and the tadpole indicates an insertion of a first order diagram. In our renormalized expansion, every two-legged insertion is subtracted at the Fermi surface, for instance, the value of the second order rainbow is

$$
Y_{2}(q)=\int d p\langle q p|V| p q\rangle C\left(p_{0}, e(\mathbf{p})\right)^{2}\left(Y_{1}\left(p_{0}, \mathbf{p}\right)-Y_{1}(0, \mathbf{P}(\mathbf{p}))\right)
$$

where $\mathbf{P}$ is the projection onto the Fermi surface defined in Lemma 2.1 of I. As in first order, the contribution of the tadpole and rainbow graphs is $C^{k h}$ by $(H 1)_{k, h}$. The contribution from the scale zero six-legged vertex 
is $C^{k, h}$ because the external momentum does not enter the fermion line and because the vertex function is $C^{k, h}$ by Lemma 2.3 .

We note in passing that although $\int|C(p)|^{2} d p=\infty$, the value of the second order rainbow and the second order tadpole would still be finite without renormalization because of the special structure of these graphs, by Lemma 2.42 of I. So divergences first appear in third order. However, the argument of Lemma 2.42 in I has an additional derivative act on $\hat{v}$, and thus one can only show that the unrenormalized value of the rainbow is $C^{k-1}$ if $\hat{v}$ is $C^{k}$.

Moreover, being conditionally convergent, the unrenormalized value depends on how the limit is taken. In particular, this graph is one of the anomalous ones of Kohn and Luttinger that prevent convergence of the unrenormalized positive temperature Green functions to their zero temperature counterparts. For the renormalized Green functions, there is no such problem. Their values at positive temperature converge to those at zero temperature. This will be shown in another paper. Here we mention this only as a further motivation why the renormalized expansion is the correct one.

The 'polarization' and 'vertex' diagram (so called because a polarization bubble, resp. a vertex correction appear as pieces of the graph) have the value (denoting $p_{k}=\left(z_{k}, \mathbf{p}_{k}\right) \in \mathbb{R} \times \mathcal{B}$ )

$$
Y(p)=\int\left(\prod_{k=1}^{3} d p_{k} C\left(z_{k}, e\left(\mathbf{p}_{k}\right)\right)\right) \delta^{\#}\left(p_{1}+p_{2}-p_{3}-p\right) P\left(p_{1}, p_{2}, p_{3}, p\right)
$$

with

$$
P\left(p_{1}, p_{2}, p_{3}, p\right)= \begin{cases}\left\langle p p_{3}|V| p_{1} p_{2}\right\rangle^{2} & \text { 'polarization' graph } \\ \left\langle p_{1} p_{2}|V| p p_{3}\right\rangle\left\langle p_{3} p|V| p_{1} p_{2}\right\rangle & \text { 'vertex' graph }\end{cases}
$$

By assumption $(H 1)_{k, h}$, the function $P$ is $C^{k, h}$ in the external momentum $p$ and in all $p_{i}$. But, whichever way one uses the delta function to express one of the $p_{i}$ in terms of $p$ and the others, that momentum will depend on $p$, so one of the propagators depends on $p$. A derivative with respect to $p$ squares the denominator and thus makes the singularity stronger, and there is no cancellation in the frequency integration as in the rainbow graph. Naive power counting suggests that already the first derivative is divergent. However, these graphs are overlapping, and therefore the bounds in I imply an improvement that makes the first derivative finite.

We repeat this proof for the example at hand to motivate the more difficult bounds that follow. First, we briefly recall the tree structure associated to the scale decomposition. If you are familiar with that, you can skip the remainder of this section. Calling

$$
\mathcal{J}=\left\{\left(j_{1}, j_{2}, j_{3}\right): I \leq j_{k}<0\right\}
$$

and inserting the scale decomposition (2.53), we get $Y(p)=\sum_{\left(j_{1}, j_{2}, j_{3}\right) \in \mathcal{J}} Y_{j_{1}, j_{2}, j_{3}}(p)$ with

$$
Y_{j_{1}, j_{2}, j_{3}}(p)=\int\left(\prod_{k=1}^{3} d p_{k} C_{j_{k}}\left(z_{k}, e\left(\mathbf{p}_{k}\right)\right)\right) \delta^{\#}\left(p_{1}+p_{2}-p_{3}-p\right) P\left(p_{1}, p_{2}, p_{3}, p\right) .
$$

At finite $I$, the integrand is bounded and $C^{k}$, so the integral for $Y$ is $C^{k}$ in $p$. All singularities arise in the limit $I \rightarrow-\infty$. We now rearrange the scale sums such that $j_{1} \leq j_{2} \leq j_{3}$. $\mathcal{J}$ is the disjoint union $\mathcal{J}=D \dot{\cup} U \dot{\cup} T$ where

$$
\begin{aligned}
D & =\{(j, j, j): j \in\{I, \ldots,-1\}\} \\
T & =\left\{\left(j_{1}, j_{2}, j_{3}\right) \in \mathcal{J}: j_{1}=j_{2} \neq j_{3} \text { or } j_{1} \neq j_{2}=j_{3} \text { or } j_{1}=j_{3} \neq j_{2}\right\} \\
U & =\left\{\left(j_{1}, j_{2}, j_{3}\right) \in \mathcal{J}: \text { if } k \neq l, \text { then } j_{k} \neq j_{l}\right\},
\end{aligned}
$$


so $Y$ is a $\operatorname{sum} Y=Y_{D}+Y_{U}+Y_{T}$ where

$$
Y_{\mathcal{M}}(p)=\sum_{\left(j_{1}, j_{2}, j_{3}\right) \in \mathcal{M}} Y_{j_{1}, j_{2}, j_{3}}(p) .
$$

For every triple $\left(j_{1}, j_{2}, j_{3}\right) \in U$, there is a unique permutation $\pi \in \mathcal{S}_{3}$ such that $j_{\pi(1)}<j_{\pi(2)}<j_{\pi(3)}$, therefore

$$
U=\left\{\left(j_{\pi(1)}, j_{\pi(2)}, j_{\pi(3)}\right): \pi \in \mathcal{S}_{3},\left(j_{1}, j_{2}, j_{3}\right) \in \Omega\right\}
$$

with

$$
\Omega=\left\{\left(j_{1}, j_{2}, j_{3}\right) \in \mathbb{Z}^{3}: I \leq j_{1}<j_{2}<j_{3}<0\right\} .
$$

Thus, calling $\underline{j}=\left(j_{1}, j_{2}, j_{3}\right)$,

$$
\begin{aligned}
Y_{U}(p) & =\sum_{\underline{j} \in \Omega} \sum_{\pi \in \mathcal{S}_{3}} \int \prod_{k=1}^{3} d p_{k} C_{j_{\pi(k)}}\left(z_{k}, e\left(\mathbf{p}_{k}\right)\right) \delta^{\#}\left(p_{1}+p_{2}-p_{3}-p\right) P\left(p_{1}, p_{2}, p_{3}, p\right) \\
& =\sum_{\underline{j} \in \Omega} \int \prod_{k=1}^{3} d p_{k} C_{j_{k}}\left(z_{k}, e\left(\mathbf{p}_{k}\right)\right) \sum_{\pi \in \mathcal{S}_{3}} \delta^{\#}\left(p_{\pi(1)}+p_{\pi(2)}-p_{\pi(3)}-p\right) P\left(p_{\pi(1)}, p_{\pi(2)}, p_{\pi(3)}, p\right)
\end{aligned}
$$

We use the $\delta^{\#}$ to 'fix' $p_{3}$, i.e. to remove the $p_{3}$-integration, and get

$$
Y_{U}(p)=\sum_{\pi \in \mathcal{S}_{3}} Y_{\Omega}^{\pi}(p)=\sum_{\pi \in \mathcal{S}_{3}} \sum_{j \in \Omega} Y_{j}^{\pi}(p)
$$

with

$$
Y_{\underline{j}}^{\pi}(p)=\int d p_{1} d p_{2} C_{j_{1}}\left(z_{1}, e\left(\mathbf{p}_{1}\right)\right) C_{j_{2}}\left(z_{2}, e\left(\mathbf{p}_{2}\right)\right) C_{j_{3}}\left(\zeta_{\pi(3)}, e_{\pi(3)}\right) P_{\pi}\left(p_{1}, p_{2}, p\right)
$$

where

$$
\zeta_{a}=L_{a}\left(z_{1}, z_{2}, z\right), \quad e_{a}=e\left(L_{a}\left(\mathbf{p}_{1}, \mathbf{p}_{2}, \mathbf{p}\right)\right)
$$

$L_{a}$ is given by

$$
L_{a}\left(p_{1}, p_{2}, p\right)= \begin{cases}p+p_{1}-p_{2} & \text { if } a=1 \\ p-p_{1}+p_{2} & \text { if } a=2 \\ -p+p_{1}+p_{2} & \text { if } a=3\end{cases}
$$

and $P_{\pi}\left(p_{1}, p_{2}, p\right)=P\left(p_{\pi(1)}, p_{\pi(2)}, p_{\pi(3)}, p\right)$ with $p_{3}$ given by $L_{\pi(3)}\left(p_{1}, p_{2}, p\right)$. For the spatial part $\mathbf{p}_{3}$, recall that the integral is over the torus $\mathcal{B}$ and that therefore the $\delta$ function is the one on the torus $\mathcal{B}$. We shall bound the $Y_{\Omega}^{\pi}$ separately for all $\pi$. The essential point is the dependence of $C_{j_{3}}$ on $p$, since $P_{\pi}$ is $C^{k, h}$ by $(H 1)_{k, h}$. We therefore redraw the graph by collapsing the interaction lines to vertices; it then looks like one of the graphs in Figure 3. The left graph corresponds to the case $a=3$ in (3.16), the right one to $a=2$.
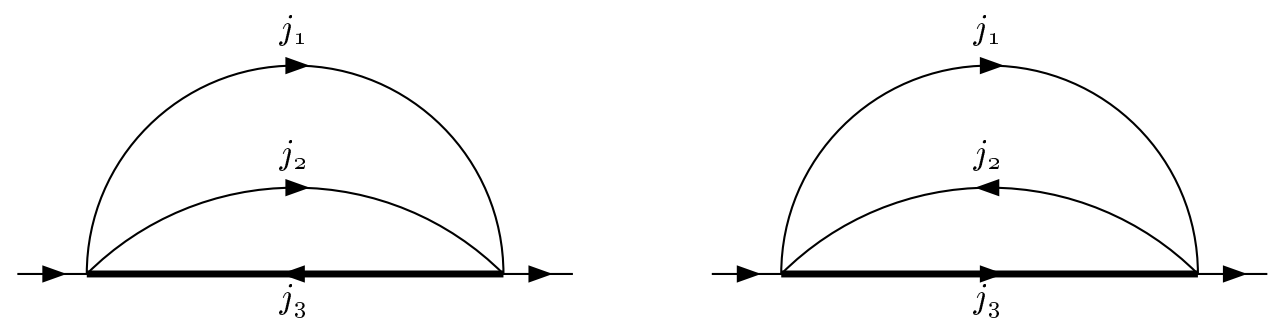

Figure 3: The sunset diagram 
Similarly, $Y_{T}$ is a sum of terms of the form

$$
\begin{gathered}
\sum_{I \leq j_{1}<j_{3}<0} \int d p_{1} d p_{2} C_{j_{1}}\left(z_{1}, e\left(\mathbf{p}_{1}\right)\right) P\left(p_{1}, p_{2}, q, p\right)\left(C_{j_{1}}\left(z_{2}, e\left(\mathbf{p}_{2}\right)\right) C_{j_{3}}(\zeta, e(\mathbf{q}))+\right. \\
\left.+C_{j_{3}}\left(z_{2}, e\left(\mathbf{p}_{2}\right)\right) C_{j_{3}}(\zeta, e(\mathbf{q}))\right)
\end{gathered}
$$

with $\zeta$ and $\mathbf{q}$ again given one of the linear combinations in (3.16), and

$$
\begin{aligned}
Y_{D}(p) & =\sum_{I \leq j<0} \int d p_{1} d p_{2}\left(C_{j}\left(z_{1}, e\left(\mathbf{p}_{1}\right)\right) C_{j}\left(z_{2}, e\left(\mathbf{p}_{2}\right)\right)\right. \\
& C_{j}\left(z_{1}+z_{2}-z, e\left(\mathbf{p}_{1}+\mathbf{p}_{2}-\mathbf{p}\right)\right) P\left(p_{1}, p_{2}, p_{1}+p_{2}-p, p\right) .
\end{aligned}
$$

Note that we have arranged that the external momentum $p$ is routed through the highest scale line $C_{j_{3}}$. The arrangement of the scale sums into sums over these sets is none other than the decomposition into Gallavotti-Nicolò trees [GN]. The trees $t$ associated to $T, D$ and $\Omega$ are drawn in Figure 4 ; the sum over all $\underline{j}$ and permutations $\pi \in \mathcal{S}_{3}$ in (3.13) corresponds to the sum over all labellings of $G$ that are consistent with $t$. Also, fixing the momentum of the line with the highest scale $j_{3}$ in terms of the others (i.e. putting that line into the spanning tree of the graph) is the natural choice for a spanning tree that is consistent with the scale structure (see Section 2.6 in I). This will be important in the estimates.

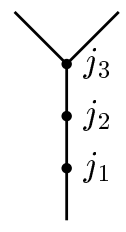

$\Omega$

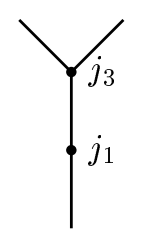

$T$

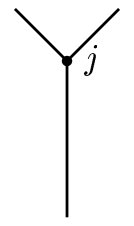

$D$

Figure 4

\subsection{Convergence Properties of Derivatives}

After these preparations, we can start to discuss convergence questions. Only $C_{j_{3}}$ depends on the external momentum $p$. Thus

$$
\left|Y_{\underline{j}}^{\pi}\right|_{s} \leq \sup _{p} \int d p_{1} d p_{2}\left|C_{j_{1}}\left(\left(p_{1}\right)_{\mathrm{o}}, e\left(\mathbf{p}_{1}\right)\right) C_{j_{2}}\left(\left(p_{2}\right)_{\mathrm{o}}, e\left(\mathbf{p}_{2}\right)\right)\right|\left|\sum_{|\alpha| \leq s}\left(\frac{\partial}{\partial p}\right)^{\alpha}\left(C_{j_{3}}\left(\zeta_{\pi(3)}, e_{\pi(3)}\right) P_{\pi}\left(p_{1}, p_{2}, p\right)\right)\right|
$$

By the Leibniz rule and (2.54),

$$
\left|Y_{\underline{j}}^{\pi}\right|_{s} \leq W_{\mathrm{o}}^{2} M^{-j_{1}-j_{2}} \sum_{r=0}^{s} \sum_{t=0}^{r} m_{r t}|P|_{r-t} W_{t} M^{-j_{3}(1+t)} \sup _{p} \int d p_{1} d p_{2} \mathbb{1}_{j_{1}}\left(p_{1}\right) \mathbb{1}_{j_{2}}\left(p_{2}\right) \mathbb{1}_{j_{3}}\left(p_{3}\right),
$$

with $m_{r t}$ a combinatorial factor, and $\mathbb{1}_{j}(p)=\mathbb{1}\left(\left|i p_{\mathrm{o}}-e(\mathbf{p})\right| \leq M^{j}\right)$. We use the coordinates $(\rho, \theta)$ and the notations $\mathbf{p}_{i}=\mathbf{p}\left(\rho_{i}, \theta_{i}\right), \mathbf{p}=\mathbf{p}(\rho, \theta), p_{i}=\left(z_{i}, \mathbf{p}_{i}\right), p=(z, \mathbf{p})$, to get

$$
\left|Y_{j}^{\pi}\right|_{s} \leq K M^{j_{1}+j_{2}-j_{3}(1+s)} \sup _{\mathbf{p}} F(\mathbf{p})
$$


with

$$
F(\mathbf{p})=\int_{\rho_{1}{ }^{2}+z_{1}{ }^{2} \leq M^{2 j_{1}}} d \rho_{1} d z_{1} \int_{\rho_{2}{ }^{2}+z_{2}{ }^{2} \leq M^{2 j_{2}}} d \rho_{2} d z_{2} \int d \theta_{1} d \theta_{2} 1\left(\left|e\left(L_{b}\left(\mathbf{p}\left(\rho_{1}, \theta_{1}\right), \mathbf{p}\left(\rho_{2}, \theta_{2}\right), \mathbf{p}\right)\right)\right| \leq M^{j_{3}}\right)
$$

and

$$
K=K\left(s,|e|_{s},|\hat{v}|_{s},|u|_{s+1}\right)=W_{\mathrm{o}}{ }^{2}|J|_{\mathrm{o}}^{2} \sum_{r \leq s} \sum_{t=0}^{r} m_{r t}\left|P_{\pi}\right|_{r-t} W_{t} .
$$

We have kept only the support function of the spatial part of the momentum in the integral for $F$. As in I, (A.2)-(A.6), we use $\max \left\{j_{1}, j_{2}\right\} \leq j_{3}$ and Taylor expansion to bound

$$
1\left(\left|e\left(L_{b}\left(\mathbf{p}\left(\rho_{1}, \theta_{1}\right), \mathbf{p}\left(\rho_{2}, \theta_{2}\right), \mathbf{p}\right)\right)\right| \leq M^{j_{3}}\right) \leq 1\left(\left|e\left(L_{b}\left(\mathbf{p}\left(0, \theta_{1}\right), \mathbf{p}\left(0, \theta_{2}\right), \mathbf{p}\right)\right)\right| \leq\left(1+2 \frac{|e|_{1}}{u_{\mathrm{o}}}\right) M^{j_{3}}\right)
$$

on the support of the integrand, and obtain $F(\mathbf{p}) \leq \mathcal{W}\left(\left(1+2 \frac{|e|_{1}}{u_{\mathrm{o}}}\right) M^{j_{3}}\right)$, where $\mathcal{W}$ is the function defined in (1.4). Thus

$$
\left|Y_{\underline{j}}^{\pi}\right|_{s} \leq \pi^{2} K M^{j_{1}+j_{2}-j_{3}(1+s)} \mathcal{W}\left(\left(1+2 \frac{|e|_{1}}{u_{\mathrm{o}}}\right) M^{j_{3}}\right) .
$$

The crude bound $\mathcal{W} \leq\left(\int d \sigma\right)^{2}$ gives the ordinary power counting bound

$$
\left|Y_{\underline{j}}^{\pi}\right|_{s} \leq \text { const } M^{j_{1}+j_{2}-j_{3}(1+s)}
$$

We now do the scale sum. In case $\Omega$, the sum is over $I \leq j_{1}<j_{2}<j_{3}<0$.

$$
\sum_{j_{3}>j_{2}} M^{-j_{3}(1+s)} \leq M^{-j_{2}(1+s)} \sum_{k \geq 0} M^{-k(1+s)}=M^{-j_{2}(1+s)} \frac{1}{M^{1+s}-1}
$$

and similarly,

$$
\sum_{j_{2}>j_{1}} M^{-j_{2}(1+s)} M^{j_{2}} \leq \begin{cases}\left|j_{1}\right| & s=0 \\ \text { const } M^{-j_{1} s} & s>0\end{cases}
$$

Since

$$
\sum_{j_{1}<0} M^{j_{1}}\left|j_{1}\right|<\infty
$$

the scale sum over the value of the undifferentiated graph $(s=0)$ is majorized by a convergent sum.

However, if $s \geq 1$,

$$
\sum_{j_{1}=I}^{-1} M^{j_{1}(1-s)} \geq \begin{cases}|I| & s=1 \\ M^{|I|(s-1)} & s \geq 2\end{cases}
$$

diverges as $I \rightarrow-\infty$. The scale sums for $T$ and $D$ behave similarly (the scale sum in $D$ is particularly simple, because there is only a sum over one scale $j$; the right side of (3.26) simply reads $M^{(1-s) j}$ ).

Using the optimal volume improvement bound, Theorem 1.1, we obtain the improved power counting bound

$$
\left|Y_{\underline{j}}^{\pi}\right|_{s} \leq \text { const } M^{j_{1}+j_{2}-j_{3} s}\left|j_{3}\right|,
$$

e.g. for $\underline{j} \in D$, the bound is now $|j| M^{(2-s) j}$. It implies that convergence holds for $s<2$. This proves that the first derivative of $\Sigma$ exists and is a continous function. For $s=2$, the sum for the bound diverges as $|I|^{2}$. 
Above, we have not given the argument why being majorized by a convergent sequence implies that the function defined by the scale sum converges as $I \rightarrow-\infty$. This is a consequence of the dominated convergence theorem, by the argument that we used in Theorem $2.46(i v)$ in I: the sequence $\left(y^{I}\right)_{I<0}$ in $\mathcal{L}=\ell^{1}\left(\mathbb{Z}_{-}, \mathcal{C}^{\circ}(\mathbb{R} \times \mathcal{B}, \mathbb{C})\right)$, given by $y^{I}=\left(y_{j}^{I}\right)_{j<0}$, where

$$
y_{j_{1}}^{I}= \begin{cases}\sum_{\substack{j_{2}, j_{3} \\ j_{3}>j_{2}>j_{1}}} Y_{j}^{\pi}(p) & j_{1} \geq I \\ 0 & \text { otherwise }\end{cases}
$$

converges pointwise in the space of sequences of continuous functions. Every element $y^{I}$ is pointwise bounded by $\left(|j| M^{j}\right)_{j<0} \in \mathcal{L}$. Thus the sequence converges to an element $\left(y_{j}\right)_{j<0}$ of $\mathcal{L}$, and $\left(\sum_{j \geq I} y_{j}^{I}\right)_{I<0}$ converges to $\sum_{j<0} y_{j}$ as $I \rightarrow-\infty$.

We have now demonstrated, for the example of the second order graph, some of the statements that hold for all two-legged 1PI graphs by the results of I and the optimal volume bound, Theorem 1.1. While too weak to make the second derivative convergent, the volume improvement given by Theorem 1.1 implies that the scale sum is only 'marginally divergent' with $|I|$, i.e. it grows only as a polynomial of $I$, not as an exponential $M^{|I|}$. Thus one may hope that with a little more care in doing the bound, the second derivative may be shown to converge. However, for the self-energy, we cannot show this. But the counterterm function $K$ depends on fewer variables than $\Sigma$, and therefore the regularity conditions on $K$ are weaker than those on $\Sigma$. More precisely, in the coordinates $(\rho, \theta)$ defined by the integral curves of $u$, derivatives with respect to $\rho$ act transversally to $S$, while derivatives with respect to $\theta$ act tangentially to $S$. From the general intuition that problems arise when the singularity is moved one may expect that the tangential derivative is better behaved than the transversal one. The counterterms are defined as

$$
K_{r}=\left(\ell \Sigma_{r}^{u n s u b}\right)(p)=\Sigma_{r}^{u n s u b}(0, \mathbf{p}(0, \theta))
$$

so they depend only on the tangential variable $\theta$, but not on $\rho$. In other words, $K$ is constant along the integral curves of $u$. It is therefore easier to check the differentiability of $K$ than that of $\Sigma$ because $p_{\mathrm{o}}$ and $\rho$ are fixed to zero in $K$ and we only have to control tangential derivatives. In the spherically symmetric case the dependence on $\theta$ drops out as well by rotational invariance, and the function is a constant. In the nonspherical case in $d=2$, the proof of regularity of $Y$ in $\theta$ is surprisingly tricky. We have

Lemma 3.1 Let $d=2$. For every $s \leq k$, there is a constant $\Delta_{s}$, depending on $|e|_{s}, g_{\mathrm{o}}$, and $w_{\mathrm{o}}$, such that for all $\underline{j}$ with $\max \left\{j_{1}, j_{2}\right\} \leq j_{3}<0$,

$$
\sup _{\theta \in S}\left|\frac{\partial^{k} Y_{\underline{j}}^{\pi}}{\partial \theta^{k}}(0, \mathbf{p}(0, \sigma))\right| \leq \Delta_{s}|P|_{s} M^{j_{1}+j_{2}} \begin{cases}M^{-j_{3}}\left|j_{3}\right| & \text { if } s \leq k-1 \\ M^{-\frac{3}{2} j_{3}}\left|j_{3}\right| & \text { if } s=k\end{cases}
$$

This Lemma immediately implies Theorem 1.2 for $d=2$ because the scale sum over $M^{j_{1}+j_{2}-\frac{3}{2} j_{3}}\left|j_{3}\right|$ converges by the above analysis. The next section contains the proof of Lemma 3.1. 


\subsection{The Singularities of the Jacobian}

In this section, we will use (H5). We choose $\theta$ as in Section 2.1, i.e. $\theta \in \mathbb{R} / 2 \pi \mathbb{Z}$ for all $\rho$, and $\left|\partial_{\theta} \mathbf{p}(0, \theta)\right|=$ const if $d=2$, and $\theta \in S^{d-1}$ for $d \geq 3$. The $\theta$-dependent part of the integral is

$$
\omega_{j, b}\left(\rho_{1}, \rho_{2}, P, p\right)=\int_{0}^{2 \pi} d \theta_{1} \int_{0}^{2 \pi} d \theta_{2} J\left(\rho_{1}, \theta_{1}\right) J\left(\rho_{2}, \theta_{2}\right) P\left(p_{1}, p_{2}, p\right) C_{j}\left(\zeta_{b}, e_{b}\right)
$$

where $\mathbf{p}_{k}=\mathbf{p}\left(\rho_{k}, \theta_{k}\right)$ and the external momentum is $p=(z, \mathbf{p}(\rho, \theta))$. $e_{b}$ and $\zeta_{b}$ given by (3.15). In this expression, the derivative with respect to $\theta$ can act on the propagator and degrade the scale behaviour of the integral, which leads to the bounds derived above. However, one may try to use a further change of variables from $\theta_{1}, \theta_{2}$ to new, $\theta$-dependent, variables to make $C_{j}$ independent of $\theta$. The integration variables $\rho_{1}$ and $\rho_{2}$ are not useful for that purpose because making $\rho_{1}$ or $\rho_{2} \theta$-dependent only transfers the dependence on $\theta$ from one of the propagators into another one in the integral (3.14) for $Y$. Then the problem that the derivative can degrade the scale behaviour arises in another part of the integrand, so nothing has been gained. A change of variables in $\theta_{1}, \theta_{2}$ puts the $\theta$-dependence only into the factors $J$ and $P$, as well as the new Jacobian. Such a change of variables is possible in most, but not all, of the integration region, because the resulting Jacobian has singularities that depend on $\theta$. The main problem in showing regularity is to control these singularities, that is, to show that the derivative does not make them nonintegrable. This requires a detailed analysis of the dependence of the singularities on $\theta$. We shall split the integral into contributions from a regular region, where the Jacobian and its derivatives are bounded, and a singular region, which contains the singularities. The regular region will be easy to treat by a change of variables. We shall show that the singular region can be chosen fixed, i.e. independent of $\theta$, if $\theta$ varies in a small neighbourhood of a given, fixed, $\theta^{(0)}$. The singularities of the Jacobian as a function of $\theta_{1}, \theta_{2}$ lie in neighbourhoods of the critical points of

$$
\eta_{b}\left(\theta_{1}, \theta_{2}, \theta\right)= \pm e\left(L_{b}\left(\mathbf{p}\left(0, \theta_{1}\right), \mathbf{p}\left(0, \theta_{2}\right), \mathbf{p}(0, \theta)\right)\right)
$$

The map $\eta_{b}$ is $C^{k}$ in all variables. After the analysis of the critical points of $\eta_{b}$, the dependence of $e\left(L_{b}\left(\mathbf{p}\left(\rho_{1}, \theta_{1}\right), \mathbf{p}\left(\rho_{2}, \theta_{2}\right), \mathbf{p}(0, \theta)\right)\right)$ on the $\rho_{i}$ will be controlled by a Taylor expansion. At the critical points,

$$
\frac{\partial \eta_{b}}{\partial \theta_{i}}\left(\theta_{1}, \theta_{2}, \theta\right)=\nabla e\left(L_{b}\left(\mathbf{p}\left(0, \theta_{1}\right), \mathbf{p}\left(0, \theta_{2}\right), \mathbf{p}(0, \theta)\right) \cdot \partial_{\theta} \mathbf{p}\left(0, \theta_{i}\right)=0 \text { for } i=1,2\right.
$$

Lemma 3.2 Let $d \geq 2$. Assume $(\mathrm{H} 2)_{2,0},(\mathrm{H} 3)$ and $(\mathrm{H} 5)$. Then all solutions $\left(\theta_{1}, \theta_{2}\right)$ of the critical point equation (3.37) for which $L_{b}\left(\mathbf{p}_{1}, \mathbf{p}_{2}, \mathbf{p}(0, \theta)\right) \in S$ holds are given by $\left(\theta_{1}^{*}, \theta_{2}^{*}\right)=c_{b, l}(\theta)$, with

$$
\begin{aligned}
& c_{b, 1}(\theta)=(\theta, \theta) \\
& c_{b, 2}(\theta)= \begin{cases}(a(\theta), \theta) & b=1,3 \\
(\theta, a(\theta)) & b=2\end{cases} \\
& c_{b, 3}(\theta)= \begin{cases}(a(\theta), a(\theta)) & b=1,2 \\
(\theta, a(\theta)) & b=3\end{cases}
\end{aligned}
$$

Here a denotes the antipodal map on the Fermi surface $S$, see (2.34). 
Proof: Denote $\mathbf{p}_{i}=\mathbf{p}\left(0, \theta_{i}\right)$ and $\mathbf{Q}=L_{b}\left(\mathbf{p}_{1}, \mathbf{p}_{2}, \mathbf{p}\right)=\mathbf{p}\left(0, \theta^{\prime}\right)$. Then

$$
\begin{array}{lll}
\nabla e(\mathbf{Q}) \cdot \partial_{\theta} \mathbf{p}\left(0, \theta_{1}\right)=0 & \Longrightarrow & \mathbf{p}_{1} \in\{\mathbf{Q}, \mathbf{a}(\mathbf{Q})\} \\
\nabla e(\mathbf{Q}) \cdot \partial_{\theta} \mathbf{p}\left(0, \theta_{2}\right)=0 & \Longrightarrow & \mathbf{p}_{2} \in\{\mathbf{Q}, \mathbf{a}(\mathbf{Q})\}
\end{array}
$$

so in particular, $\theta_{2}=\theta_{1}$ or $\theta_{2}=a\left(\theta_{1}\right)$. Let $b=1$. Then $\mathbf{p}=\mathbf{Q}-\mathbf{p}_{1}+\mathbf{p}_{2}$. Thus, given $\theta_{1}$, we have the table

\begin{tabular}{|l|c|c|}
\hline$\theta_{2}$ & $\theta^{\prime}$ & $\mathbf{p}$ \\
\hline$\theta_{1}$ & $\theta_{1}$ & $\mathbf{p}_{1}$ \\
$\theta_{1}$ & $a\left(\theta_{1}\right)$ & $\mathbf{a}\left(\mathbf{p}_{1}\right)$ \\
$a\left(\theta_{1}\right)$ & $\theta_{1}$ & $\mathbf{a}\left(\mathbf{p}_{1}\right)$ \\
$a\left(\theta_{1}\right)$ & $a\left(\theta_{1}\right)$ & $2 \mathbf{a}\left(\mathbf{p}_{1}\right)-\mathbf{p}_{1}$ \\
\hline
\end{tabular}

Given $\theta$, the last column fixes $\theta_{1}$, and the first one then fixes $\theta_{2}$. The first three rows produce the critical points $c_{1,1}, c_{1,3}$ and $c_{1,2}$. The last row drops out because, calling $\mathbf{q}=\mathbf{a}\left(\mathbf{p}_{1}\right)$ and $\mathbf{r}=\mathbf{p}_{1}$, Lemma 2.1 implies that $\mathbf{q}=\mathbf{r}$, which is a contradiction. The case $b=2$ is gotten from $b=1$ by exchanging $\theta_{1}$ and $\theta_{2} . b=3$ has $\mathbf{p}=-\mathbf{Q}+\mathbf{p}_{1}+\mathbf{p}_{2}$. The solution for $c_{3}(\theta)$ comes from the case $\mathbf{Q}=\mathbf{a}(\mathbf{p})$ (and $\theta_{2}=a\left(\theta_{1}\right)$ ).

\section{The significance of the filling condition}

The significance of this Lemma and of $(\mathrm{H} 5)$ in its proof is: given $\mathbf{p}(0, \theta)$, at the critical points, $\theta_{1}$ and $\theta_{2}$ take the values $\theta$ or $a(\theta)$. The derivative $\frac{\partial \eta}{\partial \theta_{i}}=0$, but by Lemma $3.2, \frac{\partial \eta}{\partial \theta}=0$ as well at these points. Therefore, the equation to make $\eta$ independent of $\theta$ by a change in $\theta_{1}$ and $\theta_{2}$,

$$
\frac{\partial}{\partial \theta} \eta\left(\theta_{1}(\theta), \theta_{2}(\theta), \theta\right)=0,
$$

remains consistent at these points, so a solution may still exist (and will be shown to exist below). This is not the case for the radial derivative, which is nonzero at the critical points.

Note that the collinearity argument could not have been applied without (H5) because if $2 \mathbf{a}\left(\mathbf{p}_{1}\right)-\mathbf{p}_{1}$ is on a copy of $S$ obtained by translation by some $\gamma \in \Gamma^{\#}$, the three vectors could differ (see Figure 5) for particular values of $\mathbf{p}$. In general, at these points, (3.40) has no nonsingular solution for $\frac{\partial \theta_{k}}{\partial \theta}$. This means that the derivative with respect to $\theta$ really acts on the denominator and degrades the scale behaviour. The existence of the second derivative would then require another cancellation. A sketch of this situation for the case where (Sy) holds, so that $2 \mathbf{a}\left(\mathbf{p}_{1}\right)-\mathbf{p}_{1}=-3 \mathbf{p}_{1}$ is given in Figure 5. To visualize the situation, we have drawn a periodic picture on $\mathbb{R}^{2}$ instead of a torus. The copies of $S$ obtained by translation by vectors in $\Gamma^{\#}$ are to be identified with $S$. $\frac{\partial \eta}{\partial \theta} \neq 0$ because $\partial_{\theta} \mathbf{p}$ is not parallel to $\partial_{\theta} \mathbf{p}\left(0, \theta^{\prime}\right)=-\partial_{\theta} \mathbf{p}\left(0, \theta_{1}\right)$.

In the Hubbard model, (H5) gives the filling restriction stated in the Introduction. As mentioned, such a filling restriction is not as unnatural as it may look because numerical results indicate a value of the filling below half-filling, where the Fermi surface stays fixed. This filling corresponds to $\mu=1 / 2$, which is the first value of $\mu$ where $2 S$ touches a translate of $S$.

Lemma 3.3 (i) There is $\delta=\delta\left(w_{\mathrm{o}}\right)>0$ such that for all $l, b \in\{1,2,3\}$ and for all $\theta^{(0)} \in \mathbb{R} / 2 \pi \mathbb{Z}$, there is a $C^{k-1}$-diffeomorphism

$$
\begin{aligned}
\Gamma_{b l}: U_{3 \delta}(0,0) \times U_{\delta}\left(\theta^{(0)}\right) & \rightarrow \Gamma_{b l}\left(U_{3 \delta}(0,0) \times U_{\delta}\left(\theta^{(0)}\right)\right) \subset \mathbb{R}^{2} \times U_{\delta}\left(\theta^{(0)}\right) \\
\left(t_{1}, t_{2}, \theta\right) & \mapsto(x, y, \theta)=\Gamma_{b l}\left(t_{1}, t_{2}, \theta\right),
\end{aligned}
$$




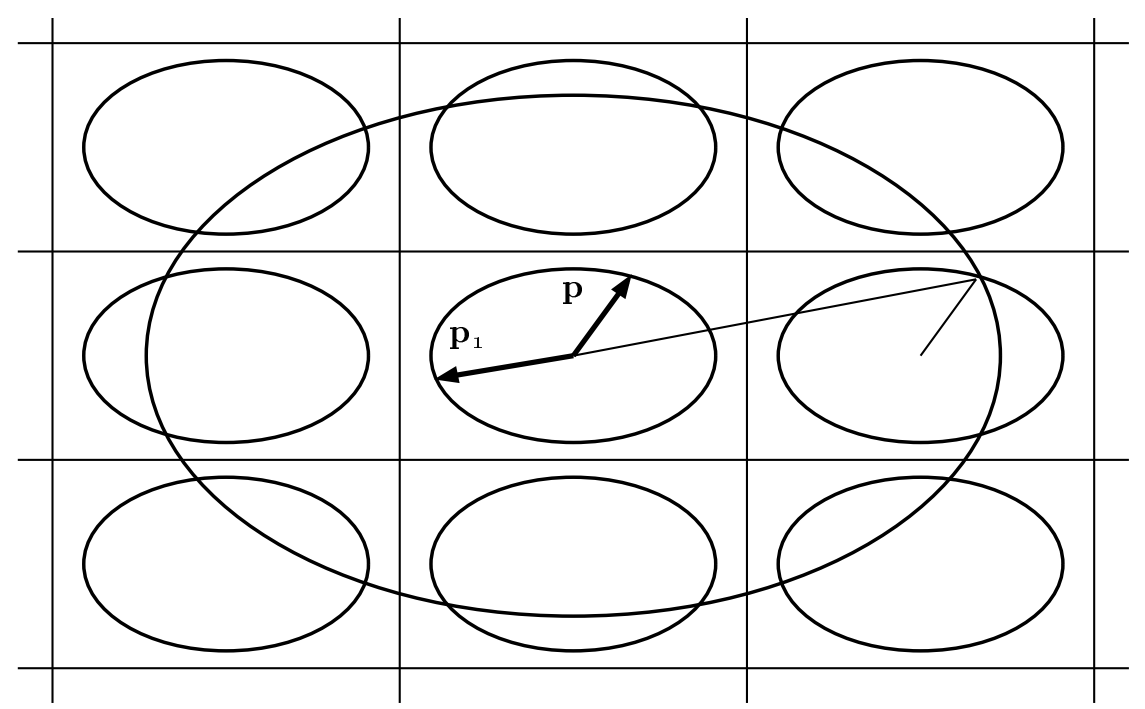

Figure 5

and a map $m_{b l}: \Gamma_{b l}\left(U_{3 \delta}(0,0)\right) \times U_{\delta}\left(\theta^{(0)}\right) \rightarrow \mathbb{R}$, with the following properties:

$$
\eta_{b}\left(c_{b l}(\theta)+\left(t_{1}, t_{2}\right), \theta\right)=m_{b l}(x, y, \theta) x y
$$

$m_{b l}$ is $C^{k-2}$, and the maps $(x, y, \theta) \mapsto x m_{b l}(x, y, \theta)$ and $(x, y, \theta) \mapsto y m_{b l}(x, y, \theta)$ are $C^{k-1}$ in all variables. Moreover, for all $\left.(x, y, \theta) \in \Gamma_{b l}\left(U_{3 \delta}(0,0)\right) \times U_{\delta}\left(\theta^{(0)}\right)\right)$,

$$
\left|m_{b l}(x, y, \theta)\right| \geq \frac{w_{\mathrm{o}}}{2}
$$

and

$$
\operatorname{det} \Gamma_{b l}^{\prime}=\left|\begin{array}{ll}
\frac{\partial x}{\partial t_{1}} & \frac{\partial x}{\partial t_{2}} \\
\frac{\partial y}{\partial t_{1}} & \frac{\partial y}{\partial t_{2}}
\end{array}\right|=1
$$

(ii) For $\varepsilon>0$ and $\left(t_{1}, t_{2}\right) \in U_{3 \delta}(0,0)$,

$$
\left|\eta_{b}\left(c_{b l}(\theta)+\left(t_{1}, t_{2}\right), \theta\right)\right|<\varepsilon
$$

implies

$$
|x y|<\frac{2 \varepsilon}{w_{\mathrm{o}}}
$$

and there are constants $Q_{s}$, depending only on $w_{\mathrm{o}}, r_{\mathrm{o}}$ and $g_{\mathrm{o}}$, and $|e|_{s}$, such that

$$
\begin{aligned}
\left|\left[\frac{\partial^{s}}{\partial \theta^{s}} \eta_{b}\right]_{x, y}\right| & \leq Q_{s}|e|_{s+2} \varepsilon \quad \text { for all } s \leq k-2 \\
\left|\left[\frac{\partial^{k-1}}{\partial \theta^{k-1}} \eta_{b}\right]_{x, y}\right| & \leq Q_{k}|e|_{k} \varepsilon^{\frac{1}{2}}
\end{aligned}
$$

In $(3.47),\left[\frac{\partial}{\partial \theta}\right]_{x, y}$ is the partial derivative with respect to $\theta$ with $x, y$ held fixed, i.e.

$$
\left[\frac{\partial^{s}}{\partial \theta^{s}} \eta_{b}\right]_{x, y}=\frac{\partial^{s}}{\partial \theta^{s}} \eta_{b}\left(\left(c_{b l}(\theta), 0\right)+\Gamma_{b l}^{-1}(x, y, \theta)\right)
$$


Proof: (i) Symmetric Case: We assume (Sy) to hold. Then the antipodal map is $a(\theta)=\theta+\pi$, hence $C^{\infty}$, and by (Sy) and a transformation $\mathbf{p}_{1} \rightarrow-\mathbf{p}_{1}$ or $\mathbf{p}_{2} \rightarrow-\mathbf{p}_{2}$, we can always choose the signs such as to make $e_{b}=e\left(\mathbf{p}_{1}+\mathbf{p}_{2}-\mathbf{p}\right)$. The critical points are then given by $b=3$ in (3.38). We define

$$
f_{l}\left(u_{1}, u_{2}, \theta\right)= \begin{cases}e\left(\mathbf{p}\left(\theta+u_{1}\right)+\mathbf{p}\left(\theta+u_{2}\right)-\mathbf{p}(\theta)\right) & l=1 \\ e\left(\mathbf{p}\left(a(\theta)+u_{1}+u_{2}\right)+\mathbf{p}\left(\theta+u_{2}\right)-\mathbf{p}(\theta)\right) & l=2 \\ e\left(\mathbf{p}\left(\theta+u_{1}\right)+\mathbf{p}\left(a(\theta)+u_{1}+u_{2}\right)-\mathbf{p}(\theta)\right) & l=3\end{cases}
$$

SO

$$
\begin{array}{ll}
f_{l}\left(0, u_{2}, \theta\right)=0 & \text { for all } u_{2}, \theta \\
f_{l}\left(u_{1}, 0, \theta\right)=0 & \text { for all } u_{1}, \theta
\end{array}
$$

In the cases $l=2$ and $l=3$, we use that by $(S y)$

$$
\mathbf{p}(a(\theta)+u)=\mathbf{p}(a(\theta+u))=-\mathbf{p}(\theta+u) .
$$

Since $a \in C^{\infty}, f_{l}$ is $C^{k}$ in all variables. By Taylor expansion,

$$
f_{l}\left(u_{1}, u_{2}, \theta\right)=u_{1} u_{2} m_{l}\left(u_{1}, u_{2}, \theta\right)
$$

with the $C^{k-2}$ function

$$
m_{l}\left(u_{1}, u_{2}, \theta\right)=\int_{0}^{1} d \alpha \int_{0}^{1} d \beta \partial_{1} \partial_{2} f\left(\alpha u_{1}, \beta u_{2}, \theta\right)
$$

At $\left(u_{1}, u_{2}\right)=(0,0)$,

$$
m_{l}(0,0, \theta)= \begin{cases}w(\mathbf{p}(\theta)) & \mathrm{l}=1 \\ -w(\mathbf{p}(a(\theta))) & \mathrm{l}=2,3\end{cases}
$$

so $\left|m_{l}(0,0, \theta)\right| \geq w_{\mathrm{o}}$ for all $\theta$. Since $k \geq 2, m_{l}$ is continuous, so there is $\delta>0$ such that for all $\theta$ and for all $\left(u_{1}, u_{2}\right) \in U_{3 \delta}(0,0),\left|m_{l}\left(u_{1}, u_{2}, \theta\right)\right| \geq \frac{w_{o}}{2}$. Also,

$$
u_{1} m_{l}\left(u_{1}, u_{2}, \theta\right)=\int_{0}^{1} d \beta \partial_{2} f_{l}\left(u_{1}, \beta u_{2}, \theta\right)
$$

is $C^{k-1}$, and similarly $u_{2} m_{l}$ is $C^{k-1}$. Let $m_{b l}=m_{l}, x=u_{1}$ and $y=u_{2}$. For $l=1, \Gamma_{b l}$ is the identity. For $l=2, \Gamma_{b l}\left(t_{1}, t_{2}, \theta\right)=\left(t_{1}-t_{2}, t_{2}, \theta\right)$, and for $l=3, \Gamma_{b l}\left(t_{1}, t_{2}, \theta\right)=\left(t_{1}, t_{2}-t_{1}, \theta\right)$. In all cases, (3.44) is obvious.

Nonsymmetric case: The change here is that the antipodal map $a \in C^{k-1}$, and that the map $\Gamma$ will now be nontrivial if the antipode is involved. We consider the $c_{b l}$ separately. The case $l=1$ is identical to the symmetric case, and all other cases are similar either to $c_{22}$, where

$$
f\left(t_{1}, t_{2}, \theta\right)=e\left(-\mathbf{p}\left(\theta+t_{1}\right)+\mathbf{p}\left(a(\theta)+t_{2}\right)+\mathbf{p}(\theta)\right),
$$

or to $c_{1,3}$, where

$$
\tilde{f}\left(t_{1}, t_{2}, \theta\right)=e\left(\mathbf{p}\left(a(\theta)+t_{1}\right)-\mathbf{p}\left(a(\theta)+t_{2}\right)+\mathbf{p}(\theta)\right) .
$$

We deal with $f$ first. Fix $\theta^{(0)} \in \mathbb{R} / 2 \pi \mathbb{Z}$. Obviously, $f\left(0, t_{2}, \theta\right)=0$ for all $t_{2}$ and $\theta$, but $f\left(t_{1}, 0, \theta\right)=0$ will in general hold only for $t_{1}=0$ because of the asymmetry. We construct a $C^{k-1}$ function $t_{2}=Y\left(t_{1}, \theta\right)$ such that

$$
f\left(t_{1}, Y\left(t_{1}, \theta\right), \theta\right)=0
$$


as follows. By Taylor expansion,

$$
f\left(t_{1}, t_{2}, \theta\right)=t_{1} g\left(t_{1}, t_{2}, \theta\right)
$$

with

$$
g\left(t_{1}, t_{2}, \theta\right)=\int_{0}^{1} d \alpha \partial_{1} f\left(\alpha t_{1}, t_{2}, \theta\right)
$$

in particular,

$$
g(0,0, \theta)=\partial_{1} f(0,0, \theta)=-\nabla e\left(\mathbf{p}(a(\theta)) \cdot \partial_{\theta} \mathbf{p}(\theta)=0 .\right.
$$

Thus, by a further Taylor expansion in $t_{2}$ and in $t_{1}$,

$$
g\left(t_{1}, t_{2}, \theta\right)=g\left(t_{1}, 0, \theta\right)+h\left(t_{1}, t_{2}, \theta\right) t_{2}=t_{1} \ell\left(t_{1}, \theta\right)+h\left(t_{1}, t_{2}, \theta\right) t_{2}
$$

with the $C^{k-2}$ functions

$$
h\left(t_{1}, t_{2}, \theta\right)=\int_{0}^{1} d \beta \partial_{2} g\left(t_{1}, \beta t_{2}, \theta\right)=\int_{0}^{1} d \alpha \int_{0}^{1} d \beta \partial_{2} \partial_{1} f\left(\alpha t_{1}, \beta t_{2}, \theta\right)
$$

and

$$
\ell\left(t_{1}, \theta\right)=\int_{0}^{1} d \alpha(1-\alpha) \partial_{1}^{2} f\left(\alpha t_{1}, 0, \theta\right)
$$

At $\left(t_{1}, t_{2}\right)=(0,0)$,

$$
h(0,0, \theta)=\partial_{2} \partial_{1} f(0,0, \theta)=-\left(\partial_{\theta} \mathbf{p}(a(\theta)), e^{\prime \prime}\left(\mathbf{p}(a(\theta)) \partial_{\theta} \mathbf{p}(\theta)\right)=w(\mathbf{p}(a(\theta)) \neq 0 .\right.
$$

The function $Y$ is constructed as the solution to the equation

$$
g\left(t_{1}, Y, \theta\right)=t_{1} \ell\left(t_{1}\right)+Y h\left(t_{1}, Y, \theta\right)=0 .
$$

A point where the equation holds is $\left(t_{1}, Y\right)=(0,0)$, and by $(3.62)$, and because $h$ is $C^{k-2}$, hence at least continuous since $k \geq 2$,

$$
\partial_{2} g(0,0, \theta)=\lim _{t_{2} \rightarrow 0} \frac{g\left(0, t_{2}, \theta\right)-g(0,0, \theta)}{t_{2}}=\lim _{t_{2} \rightarrow 0} h\left(0, t_{2}, \theta\right)=h(0,0, \theta) \neq 0 .
$$

So the solution to (3.66) exists by the implicit function theorem, and $Y$ inherits its differentiability properties from $g$. The crucial point is now that $g$ is $C^{k-1}$ in all variables because

$$
g\left(t_{1}, t_{2}, \theta\right)=\int_{0}^{1} d \alpha \nabla e\left(\mathbf{p}\left(a(\theta)+\alpha t_{1}\right)-\mathbf{p}\left(a(\theta)+t_{2}\right)+\mathbf{p}(\theta)\right) \cdot \partial_{\theta} \mathbf{p}\left(a(\theta)+\alpha t_{1}\right),
$$

and because $a, \nabla e$, and $\partial_{\theta} \mathbf{p}$ are all $C^{k-1}$. Thus $a \in C^{k-1}$ does not cause any loss in differentiability of $Y$. $|h|$ is uniformly continuous in $\theta$, so the size of the neighbourhood of $(0,0)$ where $Y$ is defined can be chosen uniformly in $\theta$ by compactness of $\mathbb{R} / 2 \pi \mathbb{Z}$. Thus there is $\delta_{1}>0$ and a function $Y \in C^{k-1}\left(\left(-\delta_{1}, \delta_{1}\right) \times\right.$ $\left.U_{\delta_{1}}\left(\theta^{(0)}\right), \mathbb{R}\right)$ such that for all $\theta \in U_{\delta_{1}}\left(\theta^{(0)}\right): Y(0, \theta)=0$, and $g\left(t_{1}, Y\left(t_{1}, \theta\right), \theta\right)=0$ for all $\left|t_{1}\right|<\delta_{1}$. This implies

$$
f\left(t_{1}, t_{2}, \theta\right)=t_{1}\left(t_{2}-Y\left(t_{1}, \theta\right)\right) \tilde{h}\left(t_{1}, t_{2}, \theta\right) .
$$


Choose $\delta$ such that $3 \delta \leq \delta_{1}$ and such that for all $\left(t_{1}, t_{2}\right) \in U_{3 \delta}(0,0),\left|\tilde{h}\left(t_{1}, t_{2}, \theta\right)\right| \geq \frac{w_{0}}{2}$. Define $\Gamma$ by

$$
x=t_{1}, \quad y=t_{2}-Y\left(t_{1}, \theta\right)
$$

and $m_{l}(x, y, \theta)=\tilde{h}(x, Y(x, \theta)+y, \theta)$, then

$$
\left|\begin{array}{ll}
\frac{\partial x}{\partial t_{1}} & \frac{\partial x}{\partial t_{2}} \\
\frac{\partial y}{\partial t_{1}} & \frac{\partial y}{\partial t_{2}}
\end{array}\right|=\left|\begin{array}{cc}
1 & 0 \\
-\frac{\partial Y}{\partial t_{1}} & 1
\end{array}\right|=1
$$

so $\Gamma(\cdot, \cdot, \theta)$ is a $C^{k-1}$-diffeomorphism at fixed $\theta$, and (3.44) holds. It follows from (3.59) and the properties of $Y$ that

$$
y m_{l}(x, y, \theta)=\left(t_{2}-Y\left(t_{1}, \theta\right)\right) h\left(t_{1}, t_{2}, \theta\right)=g(x, Y(x, \theta)+y, \theta)
$$

is $C^{k-1}$. Moreover, $f\left(t_{1}, Y\left(t_{1}, \theta, \theta\right)\right)=0$ implies by Taylor expansion

$$
f\left(t_{1}, t_{2}, \theta\right)=\left(t_{2}-Y\left(t_{1}, \theta\right)\right) \int_{0}^{1} d \alpha\left(\partial_{2} f\right)\left(t_{1}, \alpha t_{2}+(1-\alpha) Y\left(t_{1}, \theta\right), \theta\right) .
$$

Comparing that to (3.69), we see that

$$
x m_{l}(x, y, \theta)=\int_{0}^{1} d \alpha\left(\partial_{2} f\right)(x, Y(x, \theta)+\alpha y, \theta)
$$

is also $C^{k-1}$. This proves all statements of the Lemma for the case $f$. The case $\tilde{f}$ is similar: defining

$$
\bar{f}\left(\bar{t}_{1}, t_{2}, \theta\right)=\tilde{f}\left(t_{2}+\bar{t}_{1}, t_{2}, \theta\right),
$$

we have again that $\bar{f}\left(0, t_{2}, \theta\right)=0$ for all $t_{2}$ and all $\theta$, and we have thus reduced the case to the previous one by the change of variables $\left(t_{1}, t_{2}\right) \rightarrow\left(t_{1}-t_{2}, t_{2}\right)$. The Jacobian of this change of variables is one, so (3.44) still holds.

(ii) (3.46) is obvious from (3.45) and (3.43). The first bound in (3.47) follows because $m_{b l}$ is $C^{k-2}$ and $\left|\frac{\partial^{s}}{\partial \theta^{s}} m_{b l}\right| \leq$ const $|e|_{s+2}$. The constant depends on $w_{\mathrm{o}}, g_{\mathrm{o}}$, and $r_{\mathrm{o}}$. For the second bound, we use that $x m_{b l}$ and $y m_{b l}$ are $C^{k-1}$. This implies

$$
\left|\frac{\partial^{k-1} \eta_{b}}{\partial \theta^{k-1}}\right|=\left|x \frac{\partial^{k-1}}{\partial \theta^{k-1}} y m_{b l}(x, y, \theta)\right| \leq \text { const }|e|_{k}|x|
$$

and, exchanging $x$ and $y$,

$$
\left|\frac{\partial^{k-1} \eta_{b}}{\partial \theta^{k-1}}\right| \leq \text { const }|e|_{k}|y|
$$

Combining these two equations, we get

$$
\left|\frac{\partial^{k-1} \eta_{b}}{\partial \theta^{k-1}}\right| \leq \text { const }|e|_{k} \min \{|x|,|y|\} \leq \text { const }|e|_{k} \sqrt{|x y|}
$$

which implies (3.47).

To take the $\rho$-dependence into account, we use the following Lemma. 
Lemma 3.4 For any $\varepsilon>0$ there is $\delta>0$ such that for all $\theta^{(0)}$ and for all $\rho_{1}$ and $\rho_{2}$ with $\left|\rho_{i}\right|<\delta$ and all $\theta$ with $\left|\theta-\theta^{(0)}\right|<\delta$, all the solutions to the critical point equations

$$
\nabla e\left(L_{b}\left(\mathbf{p}\left(\rho_{1}, \theta_{1}\right), \mathbf{p}\left(\rho_{2}, \theta_{2}\right), \mathbf{p}(0, \theta)\right)\right) \cdot \partial_{\theta} \mathbf{p}\left(\rho_{i}, \theta_{i}\right)=0
$$

for $i=1,2$ are in $\varepsilon$-neighbourhoods of the critical points $c_{b, l}\left(\theta^{(0)}\right)$.

Proof: See Lemma B.3 and Remark B.4.

We now prove a more general statement that implies Lemma 3.1, and which we shall use later to bound more general graphs and also derivatives with respect to $e$. The generalization is about the $\theta$-behaviour of the propagators associated to lines. The free propagator $C_{j}$ is independent of the angular variable, that was the whole point of the above strategy of removing the dependence on the external angle from $C_{j_{3}}$. However, one can prove the same bounds for more general propagators if they depend on $\theta$ in a way that taking $\theta$-derivatives does not deteriorate their scale behaviour. The strings of two-legged subdiagrams that occur in general graphs and their derivatives with respect to $e$ have this property, i.e. they satisfy the hypotheses of the following theorem.

Theorem 3.5 Let $d=2$, and $(\mathrm{H} 2)_{k, 0}$ and $(\mathrm{H} 3)-\left(\right.$ H5)hold. Let $A \in C^{k-1}\left((\mathbb{R} \times \mathcal{N})^{3}, \mathbb{C}\right)$ and for $\nu \in\{1,2,3\}$ let $S_{1, j_{1}}^{(\nu)}, S_{2, j_{2}}^{(\nu)}, S_{3, j_{3}}^{(\nu)}$ be in $C^{k-1}(\mathbb{R} \times \mathcal{N}, \mathbb{C})$ and satisfy for $l \in\{1,2,3\}$

$$
\left|D^{\alpha} S_{l, j_{l}}^{(\nu)}(p)\right| \leq \Gamma_{l, \nu,|\alpha|} M^{-j_{l}(\nu+|\alpha|)} \mathbb{1}\left(\left|i p_{\mathrm{o}}-e(\mathbf{p})\right| \leq M^{j_{l}}\right) \quad \forall 0 \leq|\alpha| \leq k-1
$$

and for all $s \leq k-1$

$$
\left|\frac{\partial^{s}}{\partial \theta^{s}} S_{l, j_{l}}^{(\nu)}\left(p_{0}, \mathbf{p}(\rho, \theta)\right)\right| \leq \Gamma_{l, \nu, s} M^{-\nu j_{l}} \mathbb{1}\left(\left|i p_{0}-\rho\right| \leq M^{j_{l}}\right)
$$

(where the constants $\Gamma_{l, \nu, s}$ are increasing in $s$ ). Let $\underline{j}=\left(j_{1}, j_{2}, j_{3}\right)$ with $j_{1} \leq j_{2} \leq j_{3}, \underline{\nu}=\left(\nu_{1}, \nu_{2}, \nu_{3}\right)$, and

$$
X_{\underline{j}}^{b, \underline{\nu}}(p)=\int d p_{1} d p_{2} S_{1, j_{1}}^{\left(\nu_{1}\right)}\left(p_{1}\right) S_{2, j_{2}}^{\left(\nu_{2}\right)}\left(p_{2}\right) S_{3, j_{3}}^{\left(\nu_{3}\right)}\left(L_{b}\left(p_{1}, p_{2}, p\right)\right) A\left(p_{1}, p_{2}, p\right)
$$

where $L_{b}$ is defined in (3.16). Then, for all $s \leq k-1$, there is $Q_{s}>0$ such that for all $\nu_{1}, \nu_{2}, \nu_{3} \in\{1,2,3\}$ and all $b \in\{1,2,3\}$

$$
\sup _{\theta} \sup _{\left|p_{\mathrm{o}}-i \rho\right| \leq M^{j_{1}}}\left|\frac{\partial^{s}}{\partial \theta^{s}} X_{\underline{j}}^{b, \underline{\nu}}\left(p_{\mathrm{o}}, \mathbf{p}(\rho, \theta)\right)\right| \leq Q_{s}|A|_{s} M^{j_{1}\left(2-\nu_{1}\right)+j_{2}\left(2-\nu_{2}\right)} \begin{cases}\left|j_{3}\right| M^{\left(1-\nu_{3}\right) j_{3}} & \text { if } s \leq k-2 \\ \left|j_{3}\right| M^{\left(\frac{1}{2}-\nu_{3}\right) j_{3}} & \text { if } s=k-1\end{cases}
$$

The constant $Q_{s}$ depends only on $s$, the constants $\Gamma_{l, \nu, s}, r_{\mathrm{o}}, w_{\mathrm{o}}$, and $|e|_{s}$.

Proof: We introduce the coordinates $(\rho, \theta)$ such that $e(\mathbf{p}(\rho, \theta))=\rho$, write $p_{i}=\left(\omega_{i}, \mathbf{p}\left(\rho_{i}, \theta_{i}\right)\right)$, and denote $p_{3}^{(b)}=L_{b}\left(p_{1}, p_{2}, p\right)$. Then

$$
X_{\underline{j}}^{b, \underline{\nu}}(p)=\int d \omega_{1} d \rho_{1} \int d \omega_{2} d \rho_{2} Z_{\underline{j}}^{b, \underline{\nu}}\left(p, \omega_{1}, \rho_{1}, \omega_{2}, \rho_{2}\right)
$$


with

$$
Z_{\underline{j}}^{b, \underline{\nu}}=\int d \theta_{1} J\left(\rho_{1}, \theta_{1}\right) \int d \theta_{2} J\left(\rho_{2}, \theta_{2}\right) S_{1, j_{1}}^{\left(\nu_{1}\right)}\left(p_{1}\right) S_{2, j_{2}}^{\left(\nu_{2}\right)}\left(p_{2}\right) S_{3, j_{3}}^{\left(\nu_{3}\right)}\left(p_{3}^{(b)}\right) A\left(p_{1}, p_{2}, p\right)
$$

The momentum $p_{3}^{(b)}=L_{b}\left(p_{1}, p_{2}, p\right)$ defines $\omega_{3}^{(b)}, \rho_{3}$, and $\theta_{3}$ by by $p_{3}^{(b)}=\left(\omega_{3}^{(b)}, \mathbf{p}\left(\rho_{3}, \theta_{3}\right)\right)$ where

$$
\rho_{3}=e\left(L_{b}\left(\mathbf{p}_{1}, \mathbf{p}_{2}, \mathbf{p}\right)\right)
$$

and where $\theta_{3}$ is also a function of $p_{1}, p_{2}$ and $p$. In particular, both $\rho_{3}$ and $\theta_{3}$ depend on $\theta$. In view of (3.80), it is desirable to change variables in this integral, to make $\rho_{3}$ independent of $\theta$ because then the $j_{3}$-behaviour of the integral is not deteriorated by derivatives with respect to $\theta$.

Fix $b \in\{1,2,3\}$. Fix $\delta>0$ so small that it satisfies the hypothesis of Lemma 3.3, and such that the $3 \delta$-neighbourhoods of the critical points are mutually disjoint. Fix $r_{\mathrm{o}}>0$ so small that for all $\left|\rho_{i}\right|<r_{\mathrm{o}}$, all $\theta$ with $\left|\theta-\theta^{(0)}\right|<r_{\mathrm{o}}$, and all $l \in\{1,2,3\}$,

$$
\left|c_{b l}(\theta)-c_{b l}\left(\theta^{(0)}\right)\right|<\frac{\delta}{2}
$$

(this is possible by Lemma 3.4). Fix $\theta^{(0)} \in \mathbb{R} / 2 \pi \mathbb{Z}$. Denote $\underline{\Theta}=\left(\theta_{1}, \theta_{2}\right)$. Let

$$
R=(\mathbb{R} / 2 \pi \mathbb{Z})^{2} \backslash \bigcup_{l=1}^{3}\left\{\underline{\Theta}:\left|\underline{\Theta}-c_{b l}\left(\theta^{(0)}\right)\right|<\delta\right\},
$$

then every $\underline{\Theta} \in R$ has distance at least $\frac{\delta}{2}$ from the critical points. $R$ is compact, so there is $\xi=\xi(\delta)>0$ such that for all $\left|\rho_{i}\right|<r_{\mathrm{o}}$, all $\theta$ with $\left|\theta-\theta^{(0)}\right|<r_{\mathrm{o}}$, and all $\underline{\Theta} \in R$

$$
\left|\nabla e\left(L_{b}\left(\mathbf{p}\left(\rho_{1}, \theta_{1}\right), \mathbf{p}\left(\rho_{2}, \theta_{2}\right), \mathbf{p}(0, \theta)\right)\right)\right| \geq 2 \xi
$$

$\nabla e$ is uniformly continuous on that set, so $r_{\mathrm{o}}$ can be chosen so small that there is $N=N(\delta) \in \mathbb{N}$ and a partition of unity $1=\sum_{l=1}^{N} \chi_{l}$ with

$$
\chi_{l}(\underline{\Theta})=\left\{\begin{array}{ll}
1 & \left|\underline{\Theta}-c_{b l}\left(\theta^{(0)}\right)\right|<\delta \\
0 & \left|\underline{\Theta}-c_{b l}\left(\theta^{(0)}\right)\right|>2 \delta
\end{array} \quad \text { for } l \in\{1,2,3\},\right.
$$

and such that for all $l \geq 4, \underline{\Theta} \in \operatorname{supp} \chi_{l}$ implies either

$$
\inf _{\left|\rho_{1}\right|<\delta,\left|\rho_{2}\right|<\delta} \inf _{\left|\theta^{\prime}-\theta^{(0)}\right| \leq r_{\mathrm{o}}} \mid \frac{\partial}{\partial \theta_{1}} e\left(L_{b}\left(\mathbf{p}\left(\rho_{1}, \theta_{1}\right), \mathbf{p}\left(\rho_{2}, \theta_{2}\right), \mathbf{p}\left(0, \theta^{\prime}\right)\right) \mid \geq \xi\right.
$$

or

$$
\inf _{\left|\rho_{1}\right|<\delta,\left|\rho_{2}\right|<\delta} \inf _{\left|\theta^{\prime}-\theta^{(0)}\right| \leq r_{\mathrm{o}}} \mid \frac{\partial}{\partial \theta_{2}} e\left(L_{b}\left(\mathbf{p}\left(\rho_{1}, \theta_{1}\right), \mathbf{p}\left(\rho_{2}, \theta_{2}\right), \mathbf{p}\left(0, \theta^{\prime}\right)\right) \mid \geq \xi .\right.
$$

Note that the just constructed functions $\chi_{l}$ depend only on $\theta^{(0)}$ and $\delta$; they are independent of $\theta$. Note also that $\delta, \xi$, and the $\chi_{l}$ are independent of the scales $j_{1}, j_{2}, j_{3}$. Let $\theta$ be such that $\left|\theta-\theta^{(0)}\right|<r_{0}$. We insert the partition of unity in the integral for $Z$ and get

$$
Z_{\underline{j}}^{b, \underline{\nu}}=\sum_{l=1}^{N} Z_{\underline{j}, l}^{b, \underline{\nu}}
$$


where the integrand for $Z_{\underline{j}, \underline{l}}^{b, \underline{\nu}}$ is that of $Z_{\underline{j}}^{b, \underline{\nu}}$ times $\chi_{l}(\underline{\Theta})$. We do not write all the arguments $\left(p, \omega_{1}, \rho_{1}, \omega_{2}, \rho_{2}\right)$ of $Z_{\underline{j}}^{b, \underline{\nu}}$ for brevity. Note that $Z_{\underline{j}}^{b, \underline{\nu}}$ vanishes if $\left|i \omega_{1}-\rho_{1}\right|>M^{j_{1}}$ or if $\left|i \omega_{2}-\rho_{2}\right|>M^{j_{2}}$ because of the support properties of the propagators $S_{k, j_{k}}^{\left(\nu_{k}\right)}$, so that in deriving bounds below we may always assume that $\left|\omega_{k}\right|$ and $\left|\rho_{k}\right|$ are at most $M^{j_{k}}$.

For $l \geq 4$, an ordinary change of variables from $\theta_{1}$ or $\theta_{2}$ to $e$ is possible. If, for instance, for the given $l$ (3.91) holds, then we can write $\theta_{2}$ as a function of $e$ and the other variables,i.e.,

$$
\theta_{2}=\theta_{2}\left(\theta, e, \theta_{1}, \rho_{1}, \rho_{2}\right) .
$$

By the implicit function theorem, this function exists, and $\theta_{2}$ is a $C^{k}$ function of $\left(\theta, e, \theta_{1}, \rho_{1}, \rho_{2}\right)$. The Jacobian

$$
\tilde{J}\left(\theta, e, \theta_{1}, \rho_{1}, \rho_{2}\right)=\left[\left(\left(\frac{\partial e_{b}}{\partial \theta_{2}}\right)\left(L_{b}\left(\mathbf{p}\left(\rho_{1}, \theta_{1}\right), \mathbf{p}\left(\rho_{2}, \theta_{2}\right), \mathbf{p}(0, \theta)\right)\right)\right)^{-1}\right]_{\theta_{2}=\theta_{2}\left(\theta, e, \theta_{1}, \rho_{1}, \rho_{2}\right)}
$$

of this change of variables is $C^{k-1}$ in all its arguments. By (3.91), it satisfies, for all $l \leq k-1$,

$$
\left|\frac{\partial^{l} \tilde{J}}{\partial \theta^{l}}\right| \leq 2^{l} l ! \xi^{-l-1}\left(1+|\phi|_{l}\right)^{l}
$$

where $\phi(\theta)=\frac{\partial e_{b}}{\partial \theta_{2}}(\theta)$. By construction, $|\phi|_{l}$ is also bounded by a function of $|e|_{l+1}$ and $\xi$. Changing variables to $e$ in the integral for $Z$, we get

$$
\begin{gathered}
Z_{\underline{j}, l}^{b, \underline{\nu}}=\int d \theta_{1} J\left(\rho_{1}, \theta_{1}\right) \int d e J\left(\rho_{2}, \theta_{2}(\theta, e)\right) \tilde{J}(\theta, e) \chi_{l}\left(\theta_{1}, \theta_{2}(\theta, e)\right) A\left(p_{1}, p_{2}, p\right) \\
S_{1, j_{1}}^{\left(\nu_{1}\right)}\left(p_{1}\right) S_{2, j_{2}}^{\left(\nu_{2}\right)}\left(\omega_{2}, \mathbf{p}\left(\rho_{2}, \theta_{2}(\theta, e)\right)\right) S_{3, j_{3}}^{\left(\nu_{3}\right)}\left(\omega_{3}^{(b)}, \mathbf{p}\left(e, \theta_{3}^{(b)}(\theta, e)\right)\right) .
\end{gathered}
$$

Although $\theta_{2}(\theta, e)$ and $\theta_{3}^{(b)}$ also depend on $\theta_{1}, \rho_{1}$, and $\rho_{2}$, we suppressed this in the notation since this dependence is harmless in the following estimates by (3.91) and (3.95). By the assumptions on $S_{i, j_{i}}^{\left(\nu_{i}\right)}$ and $A$, and since $J \in C^{k-1}$ because $e \in C^{k}$, the integrand is $C^{k-1}$ in $\theta$. We take $s \leq k-1$ derivatives with respect to $\theta$, to get

$$
\left|\frac{\partial^{s}}{\partial \theta^{s}} Z_{\underline{j}, l}^{b, \underline{\nu}}\right| \leq \gamma M^{-j_{1} \nu_{1}-j_{2} \nu_{2}-\nu_{3} j_{3}} \mathbb{1}\left(\left|i \omega_{1}-\rho_{1}\right| \leq M^{j_{1}}\right) \quad \mathbb{1}\left(\left|i \omega_{2}-\rho_{2}\right| \leq M^{j_{2}}\right) \int d e \mathbb{1}\left(\left|i \omega_{3}^{(b)}-e\right| \leq M^{j_{3}}\right)
$$

with

$$
\begin{aligned}
\gamma & =2 \pi|J|_{0} \Gamma_{1, \nu_{1}, 0} \sum_{s_{1}+s_{2}+s_{3}+s_{4}=s} \frac{s !}{s_{1} ! s_{2} ! s_{3} ! s_{4} !} \Gamma_{2, \nu_{2}, s_{2}} \Gamma_{3, \nu_{3}, s_{3}} \sup \left|\frac{\partial^{s_{1}}}{\partial \theta^{s_{1}}}\left(J \tilde{J} \chi_{l}\right)\right| \sup \left|\frac{\partial^{s_{4}}}{\partial \theta^{s_{4}}} A\right| \\
& \leq 2 \pi|J|_{\mathrm{o}} 4^{s}|A|_{s} \Gamma_{1, \nu_{1}, s} \Gamma_{2, \nu_{2}, s} \Gamma_{3, \nu_{3}, s} \sup \left|\frac{\partial^{s_{1}}}{\partial \theta^{s_{1}}}\left(J \tilde{J} \chi_{l}\right)\right| \sup \left|\frac{\partial^{s}}{\partial \theta^{s}} A\right| .
\end{aligned}
$$

Thus, for $l \geq 4$,

$$
\left|\frac{\partial^{s}}{\partial \theta^{s}} Z_{\underline{j}, \underline{l}}^{b, \underline{\nu}}\right| \leq 2 \gamma M^{-j_{1} \nu_{1}-j_{2} \nu_{2}-\nu_{3} j_{3}} \mathbb{1}\left(\left|i \omega_{1}-\rho_{1}\right| \leq M^{j_{1}}\right) \quad \mathbb{1}\left(\left|i \omega_{2}-\rho_{2}\right| \leq M^{j_{2}}\right)
$$

and therefore

$$
\left|\int d \omega_{1} d \rho_{1} d \omega_{2} d \rho_{2} \sum_{l=4}^{N} \frac{\partial^{s}}{\partial \theta^{s}} Z_{\underline{j}, l}^{b, \underline{\nu}}\right| \leq 2 \pi^{2} N \gamma M^{\left(2-\nu_{1}\right) j_{1}+\left(2-\nu_{2}\right) j_{2}+\left(1-\nu_{3}\right) j_{3}}
$$


So the contribution from the region away from the singularities actually fulfills a better bound than (3.82) (recall that $N=N(\delta)$ is fixed independently of the scales).

We now turn to the singular region: let $l \in\{1,2,3\}$. Here the dependence of $S_{3, j_{3}}^{\left(\nu_{3}\right)}$ on $\theta$ cannot be removed, but we shall use the specific form of the singularity proven in Lemma 3.3 to give bounds. We first do a Taylor expansion in $\rho_{1}$ and $\rho_{2}$ to reduce the problem to all vectors being on the Fermi surface (if $\rho \neq 0$, we expand in $\rho_{1}-\rho$ and $\rho_{2}-\rho$ instead of $\rho_{1}$ and $\rho_{2}$. This does not make any difference because $\rho$ is assumed to be less than $M^{j_{1}}$ in the statement of the Lemma, because all constants are uniform in $\rho$ for $|\rho|<\delta$, and because we take a derivative with respect to $\theta$, and not with respect to $\rho$. We may therefore specialize to $\rho=0$ without loss of generality). This gives

$$
e\left(L_{b}\left(\mathbf{p}\left(\rho_{1}, \theta_{1}\right), \mathbf{p}\left(\rho_{2}, \theta_{2}\right), \mathbf{p}(0, \theta)\right)\right)=\eta_{b}\left(\theta_{1}, \theta_{2}, \theta\right)+\rho_{1} v_{1}+\rho_{2} v_{2}
$$

with

$$
\left.v_{i}\left(\theta, \rho_{1}, \rho_{2}, \theta_{1}, \theta_{2}\right)=\int_{0}^{1} d \alpha \nabla e\left(L_{b}\left(\mathbf{p}\left(\alpha \rho_{1}, \theta_{1}\right), \mathbf{p}\left(\alpha \rho_{2}, \theta_{2}\right), \mathbf{p}(0, \theta)\right)\right) \cdot \partial_{\rho} \mathbf{p}\left(\alpha \rho_{i}, \theta_{i}\right)\right) .
$$

$v_{1}$ and $v_{2}$ are $C^{k-1}$ in $\theta$. We change variables from $\underline{\Theta}$ to $\underline{t}=\underline{\Theta}-c_{b l}(\theta)$. The Jacobian of this change of variables is one. By construction of $\chi_{l}$, and because $\left|\theta-\theta^{(0)}\right|<r_{0}$, supp $\chi_{l} \subset U_{3 \delta}\left(c_{b l}(\theta)\right)$. Thus, we may use Lemma 3.3 to change variables from $\underline{t}$ to $x, y$. By (3.44), the Jacobian of this change of variables is again one. Calling $\left(\theta_{1}^{*}, \theta_{2}^{*}\right)=c_{b l}(\theta)$ and $\Gamma_{b l}^{-1}(x, y, \theta)=\left(t_{1}(\theta, x, y), t_{2}(\theta, x, y), \theta\right)$, the integral for $Z$ is

$$
\begin{gathered}
Z_{\underline{j}, l}^{b, \underline{\nu}}\left(\omega, \mathbf{p}(\rho, \theta), \omega_{1}, \ldots, \rho_{2}\right)=\int d x d y J\left(\rho_{1}, \theta_{1}^{*}+t_{1}(\theta, x, y)\right) J\left(\rho_{2}, \theta_{2}^{*}+t_{2}(\theta, x, y)\right) \chi_{l}\left(c_{b l}(\theta)+\underline{t}(\theta, x, y)\right) \\
A\left(p_{1}, p_{2}, p\right) S_{1, j_{1}}^{\left(\nu_{1}\right)}\left(p_{1}\right) S_{2, j_{2}}^{\left(\nu_{2}\right)}\left(p_{2}\right) S_{3, j_{3}}^{\left(\nu_{\nu_{3}}\right)}\left(\omega_{3}^{(b)}, \mathbf{p}\left(E_{b l}, \theta_{3}^{(b)}(x, y)\right)\right)
\end{gathered}
$$

with $p_{1}$ and $p_{2}$ now being rewritten in terms of $(x, y)$ in the obvious way, i.e. $\mathbf{p}_{1}=\mathbf{p}\left(\rho_{1}, \theta_{1}^{*}+t_{1}(\theta, x, y)\right)$ etc., and with

$$
E_{b l}=m_{b l}(x, y, \theta) x y+\tilde{v}_{1} \rho_{1}+\tilde{v}_{2} \rho_{2}
$$

and

$$
\tilde{v}_{i}=v_{i}\left(\theta, \rho_{1}, \rho_{2}, c_{b l}(\theta)+\underline{t}(\theta, x, y)\right) .
$$

By Lemma 3.3, the integrand is $C^{k-1}$ in $\theta$. We apply $s \leq k-1$ derivatives. $S_{1, j_{1}}^{\left(\nu_{1}\right)}$ and $S_{2, j_{2}}^{\left(\nu_{2}\right)}$ depend on $\theta$ only through their angular variables $\theta_{1}$ and $\theta_{2}$, so (3.80) applies there. Thus

$$
\begin{gathered}
\left|\frac{\partial^{s}}{\partial \theta^{s}} Z_{\underline{j}, l}^{b, \underline{\nu}}\right| \leq M^{-\nu_{1} j_{1}-\nu_{2} j_{2}} \int d x d y \sum_{s_{\mathrm{o}}+\ldots+s_{4}=s} \frac{s !}{s_{0} ! \ldots s_{4} !} \Gamma_{1,0, s_{1}} \Gamma_{2,0, s_{2}}\left|\frac{\partial^{s_{0}}}{\partial \theta^{s_{0}}}\left(J_{1} J_{2} \chi_{l} A\right)\right| \\
\quad \sup \left|\frac{\partial^{s_{4}}}{\partial \theta^{s_{4}}} A\right|\left|\frac{\partial^{s_{3}}}{\partial \theta^{s_{3}}} S_{3, j_{3}}^{\left(\nu_{3}\right)}\left(\omega_{r}^{(b)}, \mathbf{p}\left(E_{b l}, \theta_{3}^{(b)}(x, y, \theta)\right)\right)\right| .
\end{gathered}
$$

As mentioned, $\theta_{3}^{(b)}$ is $C^{k-1}$ in $\theta$ by Lemma 3.4. Whenever the $\theta$ derivative acts on $S_{3, j_{3}}^{\left(\nu_{3}\right)}$ through the dependence of $\theta_{3}^{(b)}$ on $\theta$, it acts only tangentially, so (3.80) ensures that the scale behaviour of such terms remains $M^{-j_{3} \nu_{3}}$. The dangerous terms are those where $S_{3, j_{3}}^{\left(\nu_{3}\right)}$ gets differentiated because of the dependence of $\rho_{3}$ on $\theta$, i.e. when $\partial_{E} S_{3, j_{3}}^{\left(\nu_{3}\right)}=\frac{\partial}{\partial E_{b l}} S_{3, j_{3}}^{\left(\nu_{3}\right)}$ or higher such derivatives occur, because the only bound we have for that case is (3.79). However, there are small factors in the numerator because of the following. By Lemma 3.3 (ii), and since for $i=1,2,\left|\rho_{i}\right|<M^{j_{i}} \leq M^{j_{3}}$,

$$
\left|\frac{\partial^{s} E_{b l}}{\partial \theta^{s}}\right| \leq \text { const } M^{j_{3}} \quad \text { for all } s \leq k-2
$$


and

$$
\left|\frac{\partial^{k-1} E_{b l}}{\partial \theta^{k-1}}\right| \leq \text { const } M^{\frac{j_{3}}{2}}
$$

The $\partial_{E}$ derivatives acting on the propagator $S_{3, j_{3}}^{\left(\nu_{3}\right)}$ give terms of the form

$$
\left(\partial_{E}^{n} S_{3, j_{3}}^{\left(\nu_{3}\right)}\right) \prod_{i=1}^{n}\left(\partial_{\theta}^{\ell_{i}} E_{b l}\right)
$$

with $\sum_{i=1}^{n} \ell_{i} \leq s \leq k-1$. At most one $\ell_{i}$ can be $k-1$, and this case can occur only if $s=k-1$ and $n=1$. If no $\ell_{i}$ is $k-1,(3.107)$ applies, and $\left|\frac{\partial^{s_{3}}}{\partial \theta^{s_{3}}} S_{3, j_{3}}^{\left(\nu_{3}\right)}\right|$ is bounded by

$$
\text { const } M^{-j_{3}\left(n+\nu_{3}\right)} M^{j_{3} n}=\text { const } M^{-\nu_{3} j_{3}}
$$

with the first factor coming from the $\left(\partial_{E}^{n} S_{3, j_{3}}^{\left(\nu_{3}\right)}\right)$ and the second from $n \partial_{\theta}^{\ell_{i}} E_{b l}$ 's. If one of the $\ell_{i}$ is $k-1$, then $n=1$ and (3.108) applies, and $\left|\frac{\partial^{s_{3}}}{\partial \theta^{s_{3}}} S_{3, j_{3}}^{\left(\nu_{3}\right)}\right|$ is bounded by

$$
\text { const } M^{-j_{3}\left(1+\nu_{3}\right)} M^{\frac{j_{3}}{2}}=\text { const } M^{-\left(\frac{1}{2}+\nu_{3}\right) j_{3}}
$$

with the first factor coming from the $\left(\partial_{E} S_{3, j_{3}}^{\left(\nu_{3}\right)}\right)$ and the second one from the one $\partial_{\theta}^{\ell_{i}} E_{b l}$ with $\ell_{i}=k-1$.

The volume of the $(x, y)$-integration is bounded by

$$
\begin{aligned}
\int_{\Gamma_{b l}\left(U_{3 \delta}(0,0)\right)} d x d y \mathbb{1}\left(\left|m_{b l}(\theta, x, y) x y\right| \leq M^{j_{3}}\right) & \leq \int_{\sqrt{x^{2}+y^{2}}<r_{D}} d x d y \mathbb{1}\left(|x y| \leq \frac{2}{w_{\mathrm{o}}} M^{j_{3}}\right) \\
& \leq \text { const }\left|\log r_{D}\right|\left|j_{3}\right| M^{j_{3}}
\end{aligned}
$$

where $r_{D}$ is the radius of a disk containing $\Gamma_{b l}\left(U_{3 \delta}(0,0)\right)$. This multiplies (3.109) and (3.110) by a factor $\left|j_{3}\right| M^{j_{3}}$.

Proof of Lemma 3.1 for $d=2$ : We take one derivative right away and get

$$
\begin{aligned}
\frac{\partial}{\partial \theta} Y_{\underline{j}}^{\pi}(0, \mathbf{p}(0, \theta)) & =\int d p_{1} \int d p_{2} C_{j_{1}}\left(\left(p_{1}\right)_{0}, e\left(\mathbf{p}_{1}\right)\right) C_{j_{2}}\left(\left(p_{2}\right)_{0}, e\left(\mathbf{p}_{2}\right)\right) \\
& \left(\partial_{\theta} P_{\pi}\left(p_{1}, p_{2}, p\right) C_{j_{3}}\left(\zeta_{b}, e_{b}\right)+P_{\pi}\left(p_{1}, p_{2}, p\right) \partial_{e} C_{j_{3}}\left(\zeta_{b}, e_{b}\right) \frac{\partial e_{b}}{\partial \theta}\right)
\end{aligned}
$$

where $b=\pi(3)$, and $\zeta_{b}$ and $e_{b}$ are defined in (3.15). The integrand is still $C^{k-1}$ in $\theta$. Theorem 3.5 applies to both terms. In the first term, take

$$
A\left(p_{1}, p_{2}, p\right)=\partial_{\theta} P_{\pi}\left(p_{1}, p_{2}, p\right)
$$

and $\nu_{1}=\nu_{2}=\nu_{3}=1$, and

$$
S_{3, j_{3}}^{\left(\nu_{3}\right)}(p)=C_{j_{3}}\left(p_{0}, e(\mathbf{p})\right)
$$

Obviously, then,

$$
\frac{\partial}{\partial \theta} S_{3, j_{3}}^{\left(\nu_{3}\right)}\left(p_{\mathrm{o}}, \mathbf{p}(\rho, \theta)\right)=0
$$


so (3.80) holds, and (3.79) holds with $\Gamma_{3,1, s}=W_{s}, W_{s}$ given in (2.54). So Theorem 3.5 applies and proves the bound for that term. In the second term, choose

$$
A\left(p_{1}, p_{2}, p\right)=P\left(p_{1}, p_{2}, p\right) \partial_{\theta} e_{b}
$$

and $\nu_{1}=\nu_{2}=1, \nu_{3}=2$, and

$$
S_{3, j_{3}}^{\left(\nu_{3}\right)}(p)=\left.\partial_{e} C_{j_{3}}\left(p_{0}, e\right)\right|_{e=e(\mathbf{p})} .
$$

Again, the hypotheses of Theorem 3.5 are satisfied, and the bound holds.

Remark 3.6 The above proof shows that $\delta$ depends only on $w_{\mathrm{o}}$, and $r_{\mathrm{o}}$ is chosen depending only on $\delta$ and $g_{\mathrm{o}}$. Thus these constants are fixed once $w_{\mathrm{o}}$ is given, and hence uniform on the set of $e$ 's that satisfy our hypotheses. Note also that $|e|_{k}$ does occur in the bound: the $A$ of (3.116) already contains a derivative of $e$, and $|A|_{k-1}$ appears when Theorem 3.5 is applied with $s=k-1$.

Remark 3.7 One may wonder where the consistency condition $\left(\frac{\partial \eta}{\partial \theta}=0\right.$ whenever $\left.\frac{\partial \eta}{\partial \theta_{1}}=\frac{\partial \eta}{\partial \theta_{2}}=0\right)$ enters in the above proof, and why the proof does not work for derivatives with respect to $\rho$. The problem is that one cannot prove the analogue of Lemma 3.3 (ii) for the $\rho$-derivative. Consider, e.g., the function

$$
H=e\left(\mathbf{p}\left(\rho_{1}, \theta_{1}\right)+\mathbf{p}\left(\rho_{2}, \theta_{2}\right)-\mathbf{p}(\rho, \theta)\right) .
$$

Proceeding in exactly the same way as above would mean first writing

$$
H=e\left(\mathbf{p}\left(0, \theta_{1}\right)+\mathbf{p}\left(0, \theta_{2}\right)-\mathbf{p}(\rho, \theta)\right)+\rho_{1} \tilde{v}_{1}+\rho_{2} \tilde{v}_{2} .
$$

The $\rho_{1}$ and $\rho_{2}$-terms from the Taylor expansion still provide enough small factors for (3.107) and (3.108), but the analogue of the function $m_{b l} x y$ appearing in (3.104) does not: the critical points $\theta_{1}^{*}(\rho, \theta), \theta_{2}^{*}(\rho, \theta)$ of the function $e\left(\mathbf{p}\left(0, \theta_{1}\right)+\mathbf{p}\left(0, \theta_{2}\right)-\mathbf{p}(\rho, \theta)\right)$ now depend on $\rho$ (their existence is proven in Lemma B.3), and most importantly, the function need not vanish at those critical points, so that (3.42) gets replaced by

$$
e\left(\mathbf{p}\left(0, \theta_{1}\right)+\mathbf{p}\left(0, \theta_{2}\right)-\mathbf{p}(\rho, \theta)\right)=f_{\mathrm{o}}(\rho, \theta)+\left(\theta_{1}-\theta_{1}^{*}\right)\left(\theta_{2}-\theta_{2}^{*}\right) \tilde{m}\left(\rho, \theta, \theta_{1}, \theta_{2}\right)
$$

with

$$
f_{\mathrm{o}}(\rho, \theta)=e\left(\mathbf{p}\left(0, \theta_{1}^{*}(\rho, \theta)\right)+\mathbf{p}\left(0, \theta_{2}^{*}(\rho, \theta)\right)-\mathbf{p}(\rho, \theta)\right)
$$

$\left(f_{\mathrm{o}}\right.$ is analogous to the function $f_{\mathrm{o}}(\mathbf{q})$ appearing in $\left.(B .48)\right)$. The crucial point is that the $\rho$-derivative of $f_{\mathrm{o}}$ is not small. In fact, it is near to maximal (and hence $O(1)$ ) since $\theta$ - and $\rho$-lines are transversal to each other in $\mathbf{p}$-space, so whenever $\partial_{\theta} \mathbf{p}$ is orthogonal to $\nabla e, \partial_{\rho} \mathbf{p}$ will point almost in the same direction as $\nabla e$.

The above problem cannot simply be circumvented by expanding $\rho_{1}$ and $\rho_{2}$ around $\rho$, because in

$$
H=e\left(\mathbf{p}\left(\rho, \theta_{1}\right)+\mathbf{p}\left(\rho, \theta_{2}\right)-\mathbf{p}(\rho, \theta)\right)+\left(\rho_{1}-\rho\right) \tilde{v}_{1}+\left(\rho_{2}-\rho\right) \tilde{v}_{2},
$$

the $\rho$-derivative can now also act on the prefactor of $\tilde{v}_{1}$, and $\left|v_{1}\right|$ is again not zero, but near to maximal at the $\theta$-critical points. 


\subsection{Hölder Continuity}

In this section, we prove Hölder continuity of the second order counterterm under the assumption that $\hat{v}$ and $e$ have the same properties. The main reason why we can show this additional regularity is that, by the above theorems, there is still a decay of almost $M^{j / 2}$ left in the scale sums, so the usual counting of derivatives by factors $M^{-j}$ suggests that one can still afford almost half a derivative, i.e., Hölder continuity with any exponent $\beta<\frac{1}{2}$. The proof will be a not very difficult add-on to the proof of Theorem 3.5. Basically, we use that the highest derivative can only appear linearly, take the differences required in the Hölder inequality, and use the differencing formula

$$
\prod_{i=1}^{n} \phi\left(\xi_{i}\right)-\prod_{i=1}^{n} \phi\left(\xi_{i}^{\prime}\right)=\sum_{k=1}^{n}\left(\prod_{i<k} \phi\left(\xi_{i}\right)\right)\left(\phi\left(\xi_{k}\right)-\phi\left(\xi_{k}^{\prime}\right)\right)\left(\prod_{i>k} \phi\left(\xi_{i}^{\prime}\right)\right)
$$

to reexpress this as a sum over differences of each factor in the integrand. The differences are either estimated by Taylor expansion or by the according Hölder property of $e$ and $\hat{v}$.

Theorem 3.8 Assume the hypotheses of Theorem 3.5. Let $0<\beta<\frac{1}{2}$ and assume that $e \in C^{k, \beta}$, $A \in C^{k-1, \beta}$, and $S_{l, j_{l}}^{\left(\nu_{l}\right)} \in C^{k-1, \beta}$. That is, there are constants $H_{e}(\beta)>0, H_{A}(\beta)>0$ and $H_{S}(\beta)>0$ such that for all multiindices $\alpha$ : if $|\alpha|=k$, for all $\mathbf{p}, \mathbf{p}^{\prime} \in \mathcal{B}$

$$
\left|D^{\alpha} e(\mathbf{p})-D^{\alpha} e\left(\mathbf{p}^{\prime}\right)\right| \leq H_{e}\left|\mathbf{p}-\mathbf{p}^{\prime}\right|^{\beta}
$$

and if $|\alpha|=k-1$,

$$
\left|D^{\alpha} A\left(p_{1}, p_{2}, p\right)-D^{\alpha} A\left(p_{1}^{\prime}, p_{2}^{\prime}, p^{\prime}\right)\right| \leq H_{A}\left|\left(p_{1}, p_{2}, p\right)-\left(p_{1}^{\prime}, p_{2}^{\prime}, p^{\prime}\right)\right|^{\beta},
$$

and for $\left|p-p^{\prime}\right| \leq M^{j}$,

$$
\left|D^{\alpha} S_{l, j_{l}}^{\left(\nu_{l}\right)}(p)-D^{\alpha} S_{l, j_{l}}^{\left(\nu_{l}\right)}\left(p^{\prime}\right)\right| \leq H_{S}\left|p-p^{\prime}\right|^{\beta} M^{-j_{l}\left(\nu_{l}+k-1+\beta\right)} \mathbb{1}\left(\left|i p_{\mathrm{o}}-e(\mathbf{p})\right| \leq G_{\mathrm{o}} M^{j_{l}}\right)
$$

Finally, we assume that for $\left|\rho-\rho^{\prime}\right| \leq M^{j_{l}}$ and $\left|\theta-\theta^{\prime}\right| \leq M^{j_{l}}$,

$$
\begin{aligned}
& \left|\left(\frac{\partial}{\partial \theta}\right)^{k-1} S_{l, j_{l}}^{\left(\nu_{l}\right)}\left(p_{0}, \mathbf{p}(\rho, \theta)\right)-\left(\frac{\partial}{\partial \theta}\right)^{k-1} S_{l, j_{l}}^{\left(\nu_{l}\right)}\left(p_{0}, \mathbf{p}\left(\rho^{\prime}, \theta^{\prime}\right)\right)\right| \\
& \quad \leq H_{S}\left|\mathbf{p}(\rho, \theta)-\mathbf{p}\left(\rho^{\prime}, \theta^{\prime}\right)\right|^{\beta} M^{-j_{l}\left(\nu_{l}+\beta\right)} \mathbb{1}\left(\left|i p_{\mathrm{o}}-\rho\right| \leq 4 M^{j_{l}}\right)
\end{aligned}
$$

Then the function $X_{\underline{j}}^{b, \underline{\nu}}$, defined in (3.81), satisfies: for all $\rho, \rho^{\prime}$ with $\max \left\{|\rho|,\left|\rho^{\prime}\right|\right\} \leq M^{j_{1}}$, all $\theta$ and $\theta^{\prime}$, and all $\left|p_{\mathrm{o}}\right| \leq M^{j_{1}}$,

$$
\begin{aligned}
& \left|\left(\frac{\partial}{\partial \theta}\right)^{k-1} X_{\underline{j}}^{b, \underline{\nu}}\left(p_{0}, \mathbf{p}(\rho, \theta)\right)-\left(\frac{\partial}{\partial \theta}\right)^{k-1} X_{\underline{j}}^{b, \underline{\nu}}\left(p_{0}, \mathbf{p}\left(\rho^{\prime}, \theta^{\prime}\right)\right)\right| \\
& \quad \leq \tilde{Q}\left|\mathbf{p}(\rho, \theta)-\mathbf{p}\left(\rho^{\prime}, \theta^{\prime}\right)\right|^{\beta} M^{j_{1}\left(2-\nu_{1}-\beta\right)+j_{2}\left(2-\nu_{2}\right)+j_{3}\left(\frac{1}{2}-\nu_{3}\right)}\left|j_{3}\right| .
\end{aligned}
$$

If $\nu_{1}=1$ and $\nu_{2}+\nu_{3} \leq 3$, the scale sum

$$
\sum_{\underline{j} \in \mathcal{M}}\left(\frac{\partial}{\partial \theta}\right)^{k-1} X_{\underline{j}}^{b, \underline{\nu}}
$$

converges absolutely to a uniformly Hölder continuous function $X_{\mathcal{M}}$ for $\mathcal{M}$ any of $\Omega, T, D$ (defined in (3.8)). 
Proof: Let

$$
\Gamma\left(p, p^{\prime}\right)=\left(\frac{\partial}{\partial \theta}\right)^{k-1} X_{\underline{j}}^{b, \underline{\nu}}\left(p_{\mathrm{o}}, \mathbf{p}(\rho, \theta)\right)-\left(\frac{\partial}{\partial \theta}\right)^{k-1} X_{\underline{j}}^{b, \underline{\nu}}\left(p_{\mathrm{o}}, \mathbf{p}\left(\rho^{\prime}, \theta^{\prime}\right)\right) .
$$

If $\left|\mathbf{p}-\mathbf{p}^{\prime}\right| \geq M^{j_{1}}$, then by $(3.82)$

$$
\left|\frac{\Gamma\left(p, p^{\prime}\right)}{\left|\mathbf{p}-\mathbf{p}^{\prime}\right|^{\beta}}\right| \leq M^{-\beta j_{1}} Q_{s}|A|_{s} M^{j_{1}\left(2-\nu_{1}\right)+j_{2}\left(2-\nu_{2}\right)}\left|j_{3}\right| M^{\left(\frac{1}{2}-\nu_{3}\right) j_{3}}
$$

which is the stated bound. Thus, it suffices to consider the case $\left|p-p^{\prime}\right| \leq M^{j_{1}}$ in the proof.

The regular and singular region are chosen as in the proof of Theorem 3.5. We prove the Hölder bound for the contribution from the regular region. The contribution from the singular region is bounded in exactly the same way. Let $x=\left(\rho_{1}, \rho_{2}, \omega_{1}, \omega_{2}\right)$. We take $k-1$ derivatives with respect to $\theta$ of $(3.96)$, to get

$$
\begin{gathered}
\frac{\partial^{k-1}}{\partial \theta^{k-1}} Z_{\underline{j}, l}^{b, \underline{\nu}}(p, x)=\int d \theta_{1} J\left(\rho_{1}, \theta_{1}\right) S_{1, j_{1}}^{\left(\nu_{1}\right)}\left(p_{1}\right) \int d e \sum_{s_{1}+s_{2}+s_{3}+s_{4}=k-1} \frac{(k-1) !}{s_{1} ! s_{2} ! s_{3} ! s_{4} !}\left(\frac{\partial}{\partial \theta}\right)^{s_{1}}\left(J \tilde{J} \chi_{l}\right) \\
\left(\left(\frac{\partial}{\partial \theta}\right)^{s_{4}} A\right)\left(\left(\frac{\partial}{\partial \theta}\right)^{s_{2}} S_{2, j_{2}}^{\left(\nu_{2}\right)}\left(p_{2}\right)\right)\left(\frac{\partial}{\partial \theta}\right)^{s_{3}} S_{3, j_{3}}^{\left(\nu_{3}\right)}\left(\omega_{3}^{(b)}, \mathbf{p}\left(e, \theta_{3}^{(b)}(\theta, e)\right)\right)
\end{gathered}
$$

where in the integrand $p_{1}=\left(\omega_{1}, \mathbf{p}\left(\rho_{1}, \theta_{1}\right)\right), p_{2}=\left(\omega_{2}, \mathbf{p}\left(\rho_{2}, \theta_{2}(\theta, e)\right)\right.$, and $p=(\omega, \mathbf{p}(\rho, \theta))$. We take the difference

$$
\Delta=\frac{\partial^{k-1}}{\partial \theta^{k-1}} Z_{\underline{j}, \underline{l}}^{b, \underline{\nu}}(p, x)-\frac{\partial^{k-1}}{\partial \theta^{k-1}} Z_{\underline{j}, \underline{l}}^{b, \underline{\nu}}\left(p^{\prime}, x\right)
$$

and split $Z=Z_{1}+Z_{2}$ and accordingly, $\Delta=\Delta_{1}+\Delta_{2}$, by regrouping the terms in the sum over $\left(s_{1}, \ldots, s_{4}\right)$. Those 4-tuples that have $s_{r}<k-1$ for all $r \in\{1,2,3,4\}$ contribute to $\Delta_{1}$; the others, where one of the $s_{r}$ equals $k-1$ and the others are zero, contribute to $\Delta_{2}$.

In $\Delta_{1}$, no derivative of order $k-1$ acts. Therefore we may use Taylor expansion to bound $\Delta$. It gives a factor $p-p^{\prime}$, but, of course, the derivative can now act on all $p$-dependent factors of the integrand. It produces at worst a factor bounded by const $M^{-j_{1}}$ (since some of the factors have bounded derivatives, and since in the others, we can use $j_{1} \leq j_{2} \leq j_{3}$ ). This combines with $\left|p-p^{\prime}\right| \leq M^{j_{1}}$ to

$$
M^{-j_{1}}\left|p-p^{\prime}\right|=M^{-j_{1}}\left|p-p^{\prime}\right|^{1-\beta}\left|p-p^{\prime}\right|^{\beta} \leq M^{-\beta j_{1}}\left|p-p^{\prime}\right|^{\beta}
$$

which proves (3.128) for the integral of $\Delta_{1}$ over $\omega_{1}, \rho_{1}, \omega_{2}$ and $\rho_{2}$.

In $\Delta_{2}$, we use (3.123) to rearrange the integrand for $\Delta_{2}$. The function $\phi_{k}$ appearing in the difference on the right side of (3.123) is $\frac{\partial^{k-1}}{\partial \theta^{k-1}}$ of $J, \tilde{J}, A$, or one of the $S_{r, j_{r}}^{\left(\nu_{r}\right)}$ (or a function on which $\frac{\partial^{k-1}}{\partial \theta^{k-1}} \operatorname{did}$ not act; this case is treated as in the last paragraph). Suppose it is $\tilde{J}$ (the other cases are easier). By $(3.94), \frac{\partial^{k-1}}{\partial \theta^{k-1}} \tilde{J}$ is a sum of terms of the form

$$
\frac{1}{\left(\partial e / \partial \theta_{2}\right)^{1+l}} \prod_{\mu=1}^{m} \partial_{\theta}^{r_{\mu}} \partial_{\theta_{2}} e
$$

with $l \geq 1, m \geq 1$, and where $r_{1}+\ldots+r_{m}=k-1$. If all $r_{\mu}$ are strictly less than $k-1$, a Taylor expansion, combined with (3.134), does the job. Let one of the $r_{\mu}$ equal $k-1$ (then $m=1$ and $l=2$ ). Applying (3.123) and the uniform Hölder property (3.124) of $e$ gives the factor $\left|p-p^{\prime}\right|^{\beta}$ and proves the statement. The strategy is the same for all other terms - the only change is that (3.125) and (3.126) (and in the singular region, (3.127)) are used. Convergence of the scale sum follows by doing the scale sums over $j_{3}, j_{2}$, and $j_{1}$, as in Section 3.2.

Proof of Hölder continuity of $\partial^{k} K_{2}$ : It suffices to bound the function $Y_{\underline{j}}^{\pi}$ in a way such that the scale sum still converges. Choose the functions $A$ and $S_{k, j_{k}}^{\left(\nu_{k}\right)}$ as done after (3.112). They satisfy (3.126), (3.125) and (3.127). The scale sum converges because the condition $\nu_{1}=1$, and $\nu_{2}+\nu_{3} \leq 3$ is satisfied. 
Proof of Hölder continuity of $\frac{\partial}{\partial p} \Sigma_{2}$ : This proof is a trivial variation of the previous one. Recall that by the volume bound, Theorem 1.1, we got (3.31). Applying (3.123) to the integral for $\frac{\partial}{\partial p} \Sigma_{2}$ and proceeding as above, Hölder continuity of any degree $\gamma<1$ follows by the same argument as above because setting $s=1$ in (3.31) leaves a decay factor $|j| M^{j}$ in the sum which can control almost one derivative.

\subsection{Higher Dimensions}

As in two dimensions, the part of the integrand for $Y_{j}^{\pi}$ that depends on the external momentum is $\omega_{j_{3}, b}$, as given by (3.35), only that now the integrals over $\theta_{1}$ and $\theta_{2}$ run over $S^{d-1}$ instead of $[0,2 \pi]$. As for $d=2$, we attempt a change of variables to make $C_{j_{3}}$ independent of $\mathbf{p}$. Again, this is possible in part of the integration region. Near the singularities of the Jacobian, we employ a strategy different from that of the two-dimensional case, and we actually show that not only the tangential, but also the radial derivatives of second order exist. We do not prove a statement about higher derivatives for $d \geq 3$.

We give an outline of this strategy before going into the details. The scale behaviour of $Y_{j}^{\pi}$ can be bounded by

$$
\left|Y_{\underline{j}}^{\pi}\right|_{s} \leq \text { const } M^{j_{1}+j_{2}} \max _{b}\left|\omega_{j_{3}, b}\right|_{s}
$$

The scale sum obtained by this bound will converge if we can show that

$$
\left|\omega_{j_{3}, b}\right|_{2} \leq \text { const } M^{-j_{3}(2-\varepsilon)}
$$

for some $\varepsilon>0$. The main idea is that by strict convexity, the singularities of the Jacobian on $S$ are isolated points $c_{b l}(\theta)$, and that for $d \geq 3$, they thus have codimension $d-1 \geq 2$ on $S$. We make the regular and singular regions scale-dependent. Let $\theta^{(0)} \in S^{d-1}$ be fixed and $0<\alpha<1$. Instead of the scale-independent neighbourhood given by the $\delta$ of Lemma 3.3, we take the singular region as an $M^{\alpha j_{3}}$-neighbourhood $U\left(\alpha, j_{3}\right)$ of $c_{b l}\left(\theta^{(0)}\right)$. To take derivatives, we then vary $\rho$ and $\theta$ only in $M^{\alpha j_{3}}$-neighbourhoods of 0 and $\theta^{(0)}$ (which suffices to calculate derivatives). The advantage of making $\delta$ depend on $j_{3}$ is that the smallness of $U\left(\alpha, j_{3}\right)$ provides additional small factors:

$$
\begin{aligned}
\int_{U\left(\alpha, j_{3}\right)} d \theta_{1} \int_{U\left(\alpha, j_{3}\right)} d \theta_{2}\left(\frac{\partial}{\partial p}\right)^{2} C_{j_{3}} \mid & \leq \text { const } M^{-3 j_{3}} \int_{U\left(\alpha, j_{3}\right)} d \theta_{1} \int_{U\left(\alpha, j_{3}\right)} d \theta_{2} \\
& \leq \text { const } M^{(-3+2(d-1) \alpha) j_{3}}
\end{aligned}
$$

because each of $\theta_{1}$ and $\theta_{2}$ is confined to a $(d-1)$-dimensional ball $U\left(\alpha, j_{3}\right)$ around the critical value. (3.137) will thus hold if we choose $\alpha>\frac{1}{2(d-1)}$. However, we cannot choose $\alpha$ as large as we want because the price we pay for making the neighbourhood $U$ depend on $\alpha$ is that the bound for the Jacobian and its derivatives also become scale-dependent. Since $S$ is strictly convex, the angle between the normal vectors increases at least linearly with the distance on $S$, and we can show that in the regular region, for $s \leq 1$,

$$
|\tilde{J}|_{s} \leq \text { const } M^{-\alpha j_{3}(1+2 s)}
$$

(as before, we cannot take $|\tilde{J}|_{2}$ because $\tilde{J}$ is only $C^{1}$ ). Taking one derivative before and one after the change of variables, as done previously, we get the bound

$$
\begin{aligned}
\left|\int d \theta_{1} \int d \tilde{e} \frac{\partial}{\partial \tilde{e}} C_{j_{3}}(\zeta, \tilde{e}) \frac{\partial}{\partial p} \tilde{J}\right| & \leq \text { const } M^{-2 j_{3}} M^{-3 \alpha j_{3}} \int d \theta_{1} \int d \tilde{e} \mathbb{1}\left(|\tilde{e}| \leq M^{j_{3}}\right) \\
& \leq \text { const } M^{-2 j_{3}} M^{(1-3 \alpha) j_{3}}
\end{aligned}
$$


In the detailed argument, there are more contributions, but they obey the same bound. For this contribution, (3.137) holds if $\alpha<\frac{1}{3}$. It is now obvious that this leaves no region for $\alpha$ in $d=2$. But for any $d \geq 3$, one has a window $\alpha \in\left(\frac{1}{4}, \frac{1}{3}\right)$ for $\alpha$ to obtain (3.137).

Thus, at this point, one sees that the two-dimensional case is more singular than the higher-dimensional one, although, superficially, the Fermi surface has the same codimension in all dimensions $d \geq 1$. The neighbourhood of singularities of the Jacobian does depend on the dimension.

We now fill in the details of this argument. We again have to make sure that the critical points, which depend on the external momentum $\mathbf{q}$, do not leave the fixed neighbourhood of $\mathbf{q}^{(0)}$ as long as $\left|\mathbf{q}-\mathbf{q}^{(0)}\right|<$ $M^{\alpha j_{3}}$, so that we can split the integration region in a way that does not depend on $\mathbf{q}$.

Lemma 3.9 Let $v_{\theta}, v_{\phi} \in\{ \pm 1\}$ and $\mathbf{q} \in \mathcal{B}$ be fixed, and define $\sigma_{\kappa}=\left\{\left(\rho_{\theta}, \rho_{\phi}\right):\left|\rho_{\theta}\right| \leq \kappa\right.$ and $\left.\left|\rho_{\phi}\right| \leq \kappa\right\}$ and

$$
E\left(\mathbf{q}, \rho_{\theta}, \rho_{\phi}, \theta, \phi\right)=e\left(\mathbf{q}+v_{\theta} \mathbf{p}\left(\rho_{\theta}, \theta\right)+v_{\phi} \mathbf{p}\left(\rho_{\phi}, \phi\right)\right) .
$$

(i) There exists a $\kappa>0$ such that for each fixed $\left(\rho_{\theta}, \rho_{\phi}\right) \in \sigma_{\kappa}$ and all $(\theta, \phi) \in S^{d-1} \times S^{d-1}$ with $\left|E\left(\mathbf{q}, \rho_{\theta}, \rho_{\phi}, \theta, \phi\right)\right| \leq 2 \kappa$, the equation

$$
\partial_{\theta_{i}} E=\partial_{\phi_{i}} E=0 \quad \forall i \in\{1, \ldots, d-1\}
$$

has at most four solutions $(\theta, \phi)=\left(\theta_{c r}^{b}(\mathbf{q}), \phi_{c r}^{b}(\mathbf{q})\right) . \kappa$ can be chosen so small that if there is a solution at $\rho_{\theta}=\rho_{\phi}=0$ and at a given $\mathbf{q}$, then there is a solution for all $\left(\rho_{\theta}, \rho_{\phi}, \mathbf{q}^{\prime}\right) \in \sigma_{\kappa} \times U_{\kappa}(\mathbf{q})$. The solutions are $C^{1}$ in $\mathbf{q}, \rho_{\theta}$ and $\rho_{\phi}$. There is $L>0$ such that for all $\left(\rho_{\theta}, \rho_{\phi}, \mathbf{q}^{\prime}\right) \in \sigma_{\kappa} \times U_{\kappa}(\mathbf{q})$,

$$
\left|\left(\phi_{c r}^{b}\left(\rho_{\theta}, \rho_{\phi}, \mathbf{q}^{\prime}\right), \theta_{c r}^{b}\left(\rho_{\theta}, \rho_{\phi}, \mathbf{q}^{\prime}\right)\right)-\left(\phi_{c r}^{b}\left(\rho_{\theta}, \rho_{\phi}, \mathbf{q}\right), \theta_{c r}^{b}\left(\rho_{\theta}, \rho_{\phi}, \mathbf{q}\right)\right)\right| \leq L\left|\mathbf{q}^{\prime}-\mathbf{q}\right|
$$

(ii) There are $K_{1} \geq 1$ and $K_{2} \geq 1$ such that for all $\varepsilon_{3} \leq \frac{\kappa}{2}$, all $\left(\rho_{\theta}, \rho_{\phi}, \mathbf{p}\right) \in \sigma_{\varepsilon_{3}} \times U_{\varepsilon_{3}}(\mathbf{q})$ and all $(\theta, \phi)$ with $\left|E\left(\mathbf{p}, \rho_{\theta}, \rho_{\phi}, \theta, \phi\right)\right| \leq \varepsilon_{3}$, either

$$
\sum_{i=1}^{d-1}\left(\left|\partial_{\theta_{i}} E\left(\mathbf{p}, \rho_{\theta}, \rho_{\phi}, \theta, \phi\right)\right|+\left|\partial_{\phi_{i}} E\left(\mathbf{p}, \rho_{\theta}, \rho_{\phi}, \theta, \phi\right)\right|\right) \geq \frac{\varepsilon_{3}}{K_{1}}
$$

or there is $b \in\{1, \ldots, 4\}$ such that

$$
\left|\theta-\theta_{c r}^{b}(\mathbf{q})\right| \leq K_{2} \varepsilon_{3} \quad \text { and } \quad\left|\phi-\phi_{c r}^{b}(\mathbf{q})\right| \leq K_{2} \varepsilon_{3} .
$$

Proof: See Appendix B.

Theorem 3.10 For all $d \geq 3$ and all $v_{\theta}, v_{\phi} \in\{ \pm 1\}$, there is a constant $Q_{3}$ such that for all $P \in$ $C^{2}(\mathbb{R} \times \mathcal{B}, \mathbb{C})$ with $|P|_{2}<\infty$,

$$
\begin{aligned}
\omega_{j_{3}} & =\omega_{j_{3}}\left(\zeta, \mathbf{p}, z_{\phi}, z_{\theta}, \rho_{\phi}, \rho_{\theta}, v_{\phi}, v_{\theta}, P\right) \\
& =\int_{S^{d-1}} d \theta J\left(\rho_{\theta}, \theta\right) \int_{S^{d-1}} d \phi J\left(\rho_{\phi}, \phi\right) P\left(p_{\theta}, p_{\phi},(\zeta, \mathbf{p})\right) C_{j_{3}}\left(\zeta, E\left(\mathbf{p}, \rho_{\theta}, \rho_{\phi}, \theta, \phi\right)\right)
\end{aligned}
$$


satisfies

$$
\left|\omega_{j_{3}}\right|_{2} \leq Q_{3} M^{-j_{3}(2-\gamma)}
$$

with some $\gamma \geq \frac{1}{8}$. Here the derivatives with respect to $\mathbf{p}$ can be taken in any direction (i.e., $\frac{\partial}{\partial \rho}$ is included), and the notation is

$$
p_{\phi}=\left(z_{\phi}, \mathbf{p}\left(\rho_{\phi}, \phi\right)\right), \quad p_{\theta}=\left(z_{\theta}, \mathbf{p}\left(\rho_{\theta}, \theta\right)\right)
$$

Proof: Let $\alpha \in(0,1)$, and fix $\mathbf{q} \in \mathcal{B}$. Without loss of generality, we may assume $\mathbf{q} \in \mathcal{P}_{\kappa}$. Let $\varepsilon_{3}=M^{\alpha j_{3}}$ and let $\mathbf{p}$ be such that $|\mathbf{p}-\mathbf{q}| \leq \varepsilon_{3}$. Since $\varepsilon_{3} \geq M^{j_{3}}$, the support properties of $C_{j_{3}}$ imply $\left|E\left(\mathbf{p}, \rho_{\theta}, \rho_{\phi}, \theta, \phi\right)\right| \leq \varepsilon_{3}$. As usual, we take one derivative with respect to $\mathbf{p}$ right away, and get

$$
\omega_{j_{3}, \mu}^{\prime}=\frac{\partial}{\partial p_{\mu}} \omega_{j_{3}}=\int_{S^{d-1}} d \theta J\left(\rho_{\theta}, \theta\right) \int_{S^{d-1}} d \phi J\left(\rho_{\phi}, \phi\right) \frac{\partial}{\partial p_{\mu}}\left(P\left(p_{\theta}, p_{\phi},(\zeta, \mathbf{p})\right) C_{j_{3}}\left(\zeta, E\left(\mathbf{p}, \rho_{\theta}, \rho_{\phi}, \theta, \phi\right)\right)\right)
$$

Since for a finite number of $j_{3}$ 's the bound holds trivially if the constant is chosen large enough, we can assume that $\varepsilon_{3}<\frac{\kappa}{2}$, where $\kappa$ is as in Lemma 3.9. Since $\max \left\{j_{1}, j_{2}\right\} \leq j_{3}$, the hypothesis of Lemma 3.9 (ii) is fulfilled. Let $K_{1}$ and $K_{2}$ be as in Lemma 3.9 (ii). For $\delta>0$ let

$$
\tilde{S}(\delta)=\bigcup_{b=1}^{4}\left\{(\theta, \phi):\left|\theta-\theta_{c r}^{b}(\mathbf{q})\right| \leq K_{2} \delta, \quad\left|\phi-\phi_{c r}^{b}(\mathbf{q})\right| \leq K_{2} \delta\right\}
$$

Fix $\delta$ such that the four sets in this union are disjoint. Let $j_{3}$ be so small that $\varepsilon_{3}<\delta / 2$. Split the integration region

$$
S^{d-1} \times S^{d-1}=\tilde{S}(\delta) \dot{\cup} \tilde{R}(\delta) .
$$

In $\tilde{R}(\delta)$ a change of variables as for $d=2$ is possible because we are at a fixed, scale-independent distance $\delta / 2$ from the critical points. For this reason, the Jacobian is also bounded by a constant that depends only on $\delta$, and the statement of the theorem follows as in the two-dimensional case.

The singular region is the union (3.150) of four disjoint sets, and corrrespondingly, $\omega_{j_{3}, \mu}^{\prime}=\sum_{b} \omega_{j_{3}, b, \mu}^{\prime}$. We may consider every $b$ separately. We subdivide the $\delta$-neighbourhood of the critical point further, as follows. Let $\chi_{1}, \chi_{2} \in C^{\infty}\left(\mathbb{R}_{\mathrm{o}}^{+},[0,1]\right)$ be a partition of unity on $\mathbb{R}_{\mathrm{o}}^{+}$, with $\operatorname{supp} \chi_{1}=[0,4]$ and $\operatorname{supp} \chi_{2}=$ $[1, \infty)$. Insert

$$
1=\chi_{1}\left(\frac{\left(\theta-\theta_{c r}^{b}(\mathbf{q})\right)^{2}+\left(\phi-\phi_{c r}^{b}(\mathbf{q})\right)^{2}}{\left(2 K_{2} \varepsilon_{3}\right)^{2}}\right)+\chi_{2}\left(\frac{\left(\theta-\theta_{c r}^{b}(\mathbf{q})\right)^{2}+\left(\phi-\phi_{c r}^{b}(\mathbf{q})\right)^{2}}{\left(2 K_{2} \varepsilon_{3}\right)^{2}}\right)
$$

in the integral. This gives two contributions, which we denote by $\omega_{j_{3}, b, \mu, 1}^{\prime}$ and $\omega_{j_{3}, b, \mu, 2}^{\prime}$.

Because of the factor $\chi_{1}$, the integrand for $\omega_{j_{3}, b, \mu, 1}^{\prime}$ is zero unless $\left|\theta-\theta_{c r}^{b}(\mathbf{q})\right| \leq 4 K_{2} \varepsilon_{3}$ and $\left|\phi-\phi_{c r}^{b}(\mathbf{q})\right| \leq$ $4 K_{2} \varepsilon_{3}$. Thus

$$
\begin{array}{r}
\frac{\partial}{\partial p_{\nu}} \omega_{j_{3}, b, \mu, 1}^{\prime}=\int d \theta \int d \phi J\left(\rho_{\theta}, \theta\right) J\left(\rho_{\phi}, \phi\right) \chi_{1}\left(\left(2 K_{2} \varepsilon_{3}\right)^{-2}\left(\left(\theta-\theta_{c r}^{b}(\mathbf{q})\right)^{2}+\left(\phi-\phi_{c r}^{b}(\mathbf{q})\right)^{2}\right)\right) \\
\frac{\partial^{2}}{\partial p_{\mu} \partial p_{\nu}}\left(P\left(p_{\theta}, p_{\phi},(\zeta, \mathbf{p})\right) C_{j_{3}}\left(\zeta, E\left(\mathbf{p}, \rho_{\theta}, \rho_{\phi}, \theta, \phi\right)\right)\right)
\end{array}
$$

By the properties of $P$ and (2.54), we have

$$
\begin{aligned}
\left|\frac{\partial}{\partial p_{\nu}} \omega_{j_{3}, b, \mu, 1}^{\prime}\right| & \leq \text { const } M^{-3 j_{3}} \int_{\left|\theta-\theta_{c r}^{b}(\mathbf{q})\right| \leq 4 K_{2} \varepsilon_{3}} d \theta \int_{\left|\phi-\phi_{c r}^{b}(\mathbf{q})\right| \leq 4 K_{2} \varepsilon_{3}} d \phi \\
& \leq \text { const } M^{-2 j_{3}} M^{-j_{3}+2(d-1) \alpha j_{3}}
\end{aligned}
$$


where the constant contains $|e|_{2},|P|_{2}$, and $|J|_{\mathrm{o}}{ }^{2}$.

Because of the factor $\chi_{1}$, the integrand for $\omega_{j_{3}, b, \mu, 2}^{\prime}$ vanishes unless $\left|\theta-\theta_{c r}^{b}(\mathbf{q})\right| \geq 2 K_{2} \varepsilon_{3}$ or $\left|\phi-\phi_{c r}^{b}(\mathbf{q})\right| \geq$ $2 K_{2} \varepsilon_{3}$, which, by Lemma 3.9 (ii), implies that (3.144) holds. Without loss of generality, we may assume that $\left|\partial_{\theta_{1}} E\right| \geq \frac{\varepsilon_{3}}{2 d K_{1}}$ throughout that region (otherwise subdivide into pieces where such a condition holds for $\left|\partial_{\theta_{i}} E\right|$ or $\left|\partial_{\phi_{i}} E\right|$ for some $i$ ). Change variables from $\theta_{1}$ to $\tilde{e}=E$, with $E$ given by (3.141), so that

$$
\theta_{1}=\theta_{1}\left(\mathbf{p}, \tilde{e}, \theta_{2}, \ldots, \theta_{d-1}, \phi, \rho_{\theta}, \rho_{\phi}\right) .
$$

We shall suppress the other arguments in $\theta_{1}=\theta_{1}(\mathbf{p}, \tilde{e})$ to keep the notation manageable, and write $\theta(\mathbf{p}, \tilde{e})=$ $\left(\theta_{1}(\mathbf{p}, \tilde{e}), \theta_{2}, \ldots, \theta_{d-1}\right)$. The Jacobian

$$
\tilde{J}=\frac{1}{\partial_{\theta_{1}} E\left(\mathbf{p}, \rho_{\theta}, \rho_{\phi}, \theta(\mathbf{p}, \tilde{e}), \phi\right)}
$$

is $C^{1}$ and it satisfies

$$
|\tilde{J}|_{\mathrm{o}} \leq \frac{2 d K_{1}}{\varepsilon_{3}} \leq \text { const } M^{-\alpha j_{3}}
$$

Moreover, differentiating

$$
\tilde{e}=E\left(\mathbf{p}, \rho_{\theta}, \rho_{\phi}, \theta(\mathbf{p}, \tilde{e}), \phi\right),
$$

with respect to $p_{\nu}$, we see that

$$
\frac{\partial \theta_{1}}{\partial p_{\nu}}=-\frac{\frac{\partial E}{\partial p_{\nu}}}{\frac{\partial E}{\partial \theta_{1}}}
$$

and therefore

$$
\left|\frac{\partial \theta_{1}}{\partial p_{\nu}}\right| \leq|e|_{1} \frac{2 d K_{1}}{\varepsilon_{3}} \leq \text { const } M^{-\alpha j_{3}}
$$

and

$$
\left|\frac{\partial \tilde{J}}{\partial p_{\nu}}\right| \leq \operatorname{const}\left(\frac{2 d K_{1}}{\varepsilon_{3}}\right)^{3} \leq \text { const } M^{-3 \alpha j_{3}}
$$

(this proves (3.139)). After the change of variables, we have

$$
\begin{array}{r}
\omega_{j_{3}, b, \mu, 2}^{\prime}=\int_{\mathbb{R}} d \tilde{e} \partial_{\tilde{e}} C_{j_{3}}(\zeta, \tilde{e}) \int d \phi J\left(\rho_{\phi}, \phi\right) \int d \theta_{2} \ldots d \theta_{d-1} J\left(\rho_{\theta}, \theta(\mathbf{p}, \tilde{e})\right) \frac{\partial E}{\partial p_{\mu}} \tilde{J}(\zeta, \tilde{e}) \\
P\left(p\left(\rho_{\theta}, \theta(\mathbf{p}, \tilde{e})\right), p\left(\rho_{\phi}, \phi\right),(\zeta, \mathbf{p})\right) \quad \chi_{2}\left(\frac{\left(\theta(\mathbf{p}, \tilde{e})-\theta_{c r}^{b}(\mathbf{q})\right)^{2}+\left(\phi-\phi_{c r}^{b}(\mathbf{q})\right)^{2}}{\left(2 K_{2} \varepsilon_{3}\right)^{2}}\right)
\end{array}
$$

plus a term where the derivative $\frac{\partial}{\partial p_{\mu}}$ acted on $P$, not on $C_{j_{3}}$, and which therefore obeys a bound that is by a factor $M^{j_{3}}$ better than the one we are about to prove. The second derivative $\frac{\partial}{\partial p_{\nu}}$ can now act on $\tilde{J}, \chi_{2}$, $J\left(\rho_{\theta}, \theta(\mathbf{p}, \tilde{e}), \frac{\partial E}{\partial p_{\mu}}\right.$, and $P$, but not on $C_{j_{3}}$. By $(3.157),(3.160)$, and (3.161), its effect is in all cases bounded by const $\varepsilon_{3}{ }^{-3}$. The support properties of $C_{j_{3}}$ restrict $\tilde{e}$ to an interval of length $M^{j_{3}}$. Thus

$$
\begin{aligned}
\left|\omega_{j_{3}, b, \mu, 2}^{\prime}\right| & \leq \mathrm{const} \int_{\mathbb{R}} d \tilde{e} \mathbb{1}\left(|\tilde{e}| \leq M^{j_{3}}\right) M^{-2 j_{3}} M^{-3 \alpha j_{3}} \\
& \leq \mathrm{const} M^{-2 j_{3}} M^{(1-3 \alpha) j_{3}} .
\end{aligned}
$$

To fulfill (3.147), we have to have $2 \alpha(d-1)-1>0$ and $1-3 \alpha>0$, i.e.,

$$
\frac{1}{2(d-1)}<\alpha<\frac{1}{3}
$$


Taking $\alpha=\frac{7}{24}$, we get $1-3 \alpha=\frac{1}{8}$ and $2(d-1) \alpha-1 \geq \frac{1}{6}$.

Remark 3.11 Proving Hölder continuity under the hypotheses that the second derivatives of $e$ and $\hat{v}$ are also Hölder continuous is a straightforward adaption of the proof given for $d=2$ in Section 3.4. Recall that in second order, $P$ is given by (3.5), so it fulfills the hypothesis of Theorem 3.10, and hence Theorem 1.2 (ii) follows. Using the methods of Chapter 3 of $\mathrm{I}$, one can show the same for $p_{\mathrm{o}}$-derivatives, i.e., that $\Sigma$ is $C^{2}$ in $p_{\mathrm{o}}$ for all $d \geq 3$. This requires additional integration by parts and a resummation of the partition of unity over the scales, so we will not include this proof here.

\section{The Wicked Ladders}

In this section, we generalize the results proven above to the class of graphs shown in Figure 6 . These graphs are constructed by joining two legs of a four-legged ladder diagram by a Wick line to get a two-legged diagram (although, of course, the scales associated to these lines will in general not make them Wick lines). For this reason, and for other reasons that should become clearer in the following discussion, we call them the 'wicked ladders'. Alternatively, one may call these graphs the RPA graphs since their sum contains the RPA resummation for the self-energy.

Definition 4.1 The generalized RPA self-energy $\Sigma_{\mathrm{RPA}}$ and the counterterm $K_{\mathrm{RPA}}$ are the formal power series in $\lambda$ given by the sum of all first and second order graphs and all graphs of the form shown in Figure 6 , with an arbitrary number of bubbles, and where the vertices have the vertex function $\hat{v}$ of $(2.5)$.

Thus this class of graphs includes both the contributions from those that are usually called RPA graphs and contributions from the ladders to the self-energy. The motivation for looking at these graphs is that they give the most singular contributions to the self-energy (this is proven in III). We shall prove in this section that if $e$ and $\hat{v}$ are $C^{k}$, then the same holds for tangential derivatives of the value of the wicked ladders. More precisely, for the particle-hole wicked ladder, shown in Figure $6(a)$, we show this for any $k \geq 2$. For the particle-particle wicked ladder, shown in Figure $6(b)$, we show this for all $k \geq 2$ if the Fermi surface obeys (Sy). If the Fermi surface is nonsymmetric, we prove the statement for the particle-particle wicked ladder only for $k=2$.

The method used is similar to that of the second order case: we split the integration region into a regular and a singular region, apply a simple change of variables in the regular region and analyze the critical points in the singular region in detail. This generalization turns out to be less straightforward than one might expect. For this class of graphs there is already an essential complication in the critical point analysis. Thus, the analysis of critical points is fragile in that it changes a lot if the graphs get more complicated. The method used in III to deal with all other graphs is much more robust.

Without loss of generality, we may put $\hat{v}=1$, since it is $C^{k}$ by (H1), so that terms arising when derivatives act on $\hat{v}$ are bounded uniformly in the scales by (H1). Also, we put the same scale $j$ on all the 


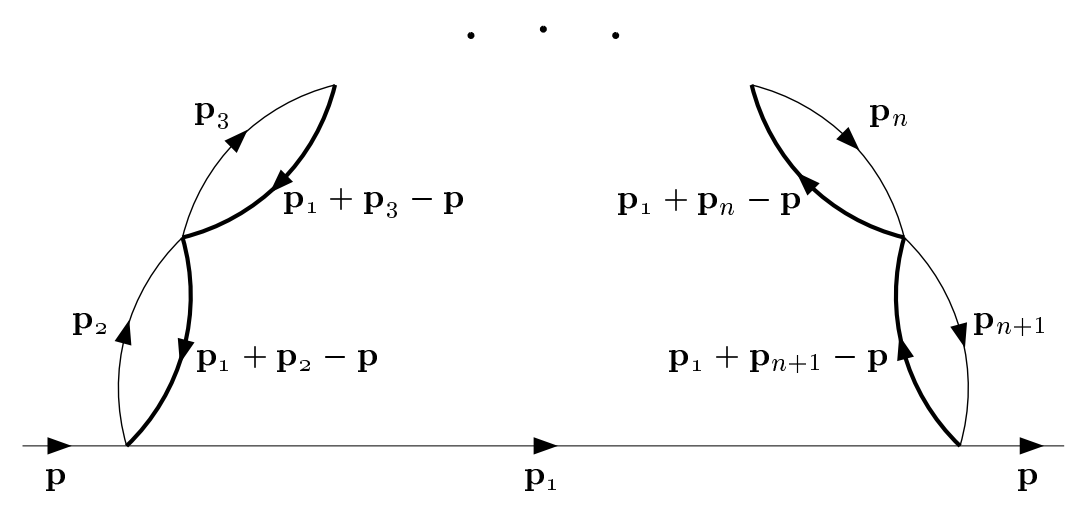

(a)

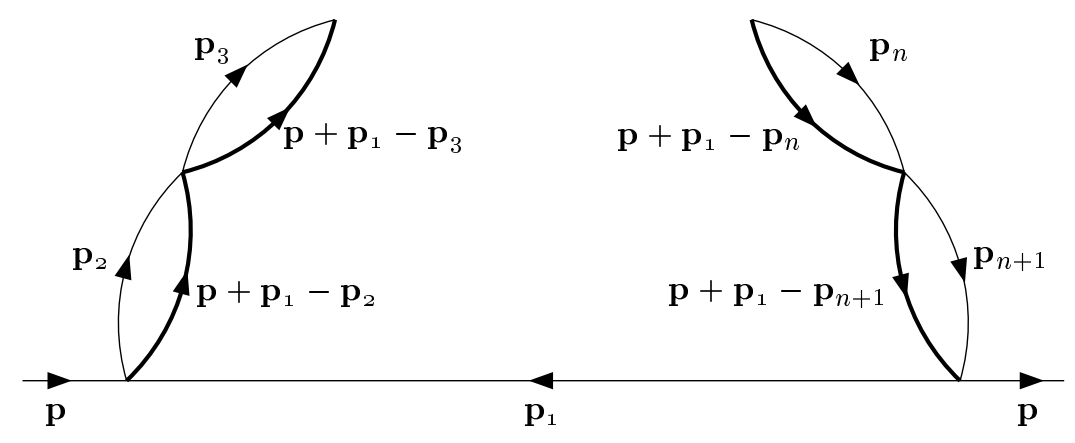

(b)

Figure 6: The wicked ladder

propagators. The value of the graph shown in Figure 6 is then

$$
\operatorname{Val}(G)(\mathbf{p})=\int\left(\prod_{r=1}^{n+1} d p_{r} C_{j}\left(\omega_{r}, e\left(\mathbf{p}_{r}\right)\right)\right) \prod_{s=2}^{n+1} C_{j}\left(\tilde{\omega}_{s}, e\left(\mathbf{t}_{s}\right)\right)
$$

with

$$
\begin{aligned}
\tilde{\omega}_{s} & = \begin{cases}\omega_{1}-\omega+\omega_{s} & \text { Fig. } 6(a) \\
\omega_{1}+\omega-\omega_{s} & \text { Fig. } 6(b)\end{cases} \\
\mathbf{t}_{s} & = \begin{cases}\mathbf{p}_{1}-\mathbf{p}+\mathbf{p}_{s} & \text { Fig. } 6(a) \\
\mathbf{p}_{1}+\mathbf{p}-\mathbf{p}_{s} & \text { Fig. } 6(b) .\end{cases}
\end{aligned}
$$

Again, the derivative with respect to the external momentum may act on propagators associated to lines of the graph (with the choice of spanning tree of Figure 6, this may affect many lines; this spanning tree is, however, convenient in the following because of its symmetry). There are $n+1$ angular variables that can be used to make changes of variables, as in the second order case. But, again, there are critical points where such a change of variables is not possible. 
To see where these critical points are, we again look first at the case where all $\mathbf{p}_{i}$ are on $S$. Let $g(\mathbf{p})=\nabla e(\mathbf{p}), \tau(\mathbf{p})=\partial_{\theta} \mathbf{p}$, and for $k \in\{2, \ldots, n+1\}$ let

$$
g_{1 k}= \begin{cases}g\left(\mathbf{p}_{1}-\mathbf{p}+\mathbf{p}_{k}\right) & \text { Fig. } 6(a) \\ g\left(\mathbf{p}_{1}+\mathbf{p}-\mathbf{p}_{k}\right) & \text { Fig. } 6(b) .\end{cases}
$$

Moreover, let $\tau_{k}=\tau\left(\mathbf{p}_{k}\right)$ and $\tau=\tau(\mathbf{p})$. Suppose we make a change of variables $\Theta=\left(\theta_{1}, \ldots, \theta_{n+1}\right)^{t}$ $\rightarrow \tilde{\Theta}(\Theta, \theta)=\left(\tilde{\theta}_{1}, \ldots, \tilde{\theta}_{n+1}\right)^{t}$ (the superscript $t$ is to mean the transpose so that these are column vectors). The condition that the propagator for the $k^{t h}$ line in the tree is independent of the external momentum $\mathbf{p}=\mathbf{p}(0, \theta)$ is

$$
\frac{\partial}{\partial \theta} e\left(\mathbf{p}\left(0, \tilde{\theta}_{1}(\Theta, \theta)\right) \mp \mathbf{p}(0, \theta) \pm \mathbf{p}\left(0, \tilde{\theta}_{k}(\Theta, \theta)\right)\right)=g_{1 k} \cdot \tau_{1} \frac{\partial \tilde{\theta}_{1}}{\partial \theta} \pm g_{1 k} \cdot \tau_{k} \frac{\partial \tilde{\theta}_{k}}{\partial \theta} \mp g_{1 k} \cdot \tau=0 .
$$

The system of equations ensuring that all propagators in the tree are independent of $\theta$ is

$$
\Gamma^{(n)}(\tilde{\Theta}(\Theta, \theta), \theta) \cdot \frac{\partial \tilde{\Theta}}{\partial \theta}=\gamma(\tilde{\Theta}(\Theta, \theta), \theta)
$$

with

$$
\Gamma^{(n)}(\Theta, \theta)=\left(\begin{array}{cccccc}
g_{12} \cdot \tau_{1} & \pm g_{12} \cdot \tau_{2} & 0 & 0 & \ldots & 0 \\
g_{13} \cdot \tau_{1} & 0 & \pm g_{13} \cdot \tau_{3} & 0 & \ldots & 0 \\
g_{14} \cdot \tau_{1} & 0 & 0 & \pm g_{14} \cdot \tau_{4} & \ldots & 0 \\
\vdots & \vdots & \vdots & & \ddots & \vdots \\
g_{1 n+1} \cdot \tau_{1} & 0 & 0 & \ldots & 0 & \pm g_{1 n+1} \cdot \tau_{n+1}
\end{array}\right)
$$

and

$$
\gamma(\Theta, \theta)= \pm\left(g_{12} \cdot \tau, \ldots, g_{1, n+1} \cdot \tau\right)^{t} .
$$

By definition, the critical points are those points where the matrix $\Gamma^{(n)}$ (which has $n$ rows and $n+1$ columns) has $\operatorname{rank}\left(\Gamma^{(n)}\right) \leq n-1$. A way from these points, (4.5) is easily solved by first setting one of $\frac{\partial \tilde{\theta}_{1}}{\partial \theta}, \ldots, \frac{\partial \tilde{\theta}_{n+1}}{\partial \theta}$ equal to zero. This effectively deletes one column from $\Gamma^{(n)}$. The resulting matrix is then invertible, and the corresponding system of differential equations is soluble with initial condition $\tilde{\theta}_{i}=\theta_{i}$ (this case is discussed in detail below).

Since one could also have chosen a different spanning tree, we briefly motivate our choice. At a first glance, it may seem a much better strategy to put the line that carries momentum $\mathbf{p}_{\mathbf{1}}$ into the spanning tree, because then only this line depends on the external momentum. This would then give, e.g. for (a), $\mathbf{p}_{1}=\mathbf{p}+\mathbf{p}_{2}-\mathbf{q}_{1}$, where $\mathbf{q}_{1}$ is now another loop momentum. Assuming that we are in the regular region, i.e. away from the critical points of $e\left(\mathbf{p}+\mathbf{p}_{2}-\mathbf{q}_{1}\right)$, one can then do the same change of variables as in the second order case to make the propagator on line 1 independent of $\mathbf{p}$. However, this time the dependence on the external $\theta$ goes not only into a Jacobian, but, through the changed variables, also into one of the propagators associated to the other lines of the spanning tree, so that the derivative w.r.t. $\theta$ may still degrade the scaling behaviour in a dangerous way. Then, one can do another change of variables to get rid of the dependence on $\theta$ of the next line in the spanning tree, etc. Although such a procedure is possible, it gets rather complicated already in the three-loop case $(n=2)$. It is much easier to look at the entire system of equations at once. The simple form of the matrix $\Gamma^{(n)}$ is due to our symmetric choice of the spanning tree.

We now classify the critical points for the system of equations (4.5). 
Remark 4.2 For $\Gamma^{(n)}$ to have rank strictly less than $n$, it is necessary that either

(1) one of columns 2 through $n+1$ vanishes, say $g_{1 k} \cdot \tau_{k}=0$, and the corresponding entry in column one, $g_{1 k} \cdot \tau_{1}$, also vanishes, or

(2) at least two of columns 2 through $n+1$ vanish.

We deal with case of the particle-hole wicked ladder (Figure $6(a)$ ) first.

Lemma 4.3 For the particle-hole wicked ladder, if rank $\Gamma^{(n)} \leq n-1$ and all momenta are on $S$ then one of the following holds.

(i) $\quad \mathbf{p}_{1}=\mathbf{p}$; then rank $\Gamma^{(n)} \leq 1$ and all columns of $\Gamma^{(n)}$ except for the first vanish.

(ii) $\mathbf{p}_{1}=\mathbf{a}(\mathbf{p})$, and there is $\emptyset \neq C \subset\{2, \ldots, n+1\}$ such that for all $k \in C, \mathbf{p}_{k}=\mathbf{p}$, and for all $l \notin C$, $\mathbf{p}_{l} \neq \mathbf{p}$; then rank $\Gamma^{(n)}=n-|C|$, and all columns with index $k \in C$ vanish: $\Gamma_{m k}^{(n)}=0$ for all $m \in\{2, \ldots, n+1\}$, all rows with index $k-1, k \in C$, vanish: $\Gamma_{k-1, m}^{(n)}=0$ for all $m \in\{1, \ldots, n\}$, all columns with index $k \in\{2, \ldots, n+1\} \backslash C$ are nonzero.

Whenever row $k$ of $\Gamma^{(n)}$ vanishes, the corresponding component $\gamma_{k}$ of the vector $\gamma$ on the right hand side of (4.6) also vanishes. In case (i), the right hand side $\gamma$ coincides with the first column of $\Gamma^{(n)}$.

Proof: We go through th cases mentioned in Remark 4.2. Case (1): The two equations $g_{1 k} \cdot \tau_{k}=0$ and $g_{1 k} \cdot \tau_{1}=0$ are precisely the system of equations discussed in the second order case, only now $\mathbf{p}_{k}$ appears instead of $\mathbf{p}_{2}$. Therefore $\mathbf{p}_{1}=\mathbf{p}_{k}=\mathbf{p}$ or $\mathbf{p}_{1}=\mathbf{a}(\mathbf{p}), \mathbf{p}_{k}=\mathbf{p}$ or $\mathbf{p}_{1}=\mathbf{p}, \mathbf{p}_{k}=\mathbf{a}(\mathbf{p})$ holds, and in particular, $\mathbf{p}_{1}-\mathbf{p}+\mathbf{p}_{k} \in\{\mathbf{p}, \mathbf{a}(\mathbf{p})\}$ so that $g_{1 k} \cdot \tau$ vanishes. If $\mathbf{p}_{1}=\mathbf{p}$, then $g_{1 \ell} \cdot \tau_{\ell}=g\left(\mathbf{p}_{\ell}\right) \cdot \tau\left(\mathbf{p}_{\ell}\right)=0$ holds for all $\ell \geq 2$, and this case is stated as item $(i)$ in the Lemma. If $\mathbf{p}_{\mathbf{1}}=\mathbf{a}(\mathbf{p})$, then $\mathbf{p}_{k}=\mathbf{p}$ and $\mathbf{p}_{1}-\mathbf{p}+\mathbf{p}_{k}=\mathbf{a}(\mathbf{p})$. Therefore, for any additional $\ell \in\{2, \ldots, n+1\}$, the vanishing of column number $\ell, g_{1 \ell} \cdot \tau_{\ell}=0$, means $g\left(\mathbf{a}(\mathbf{p})-\mathbf{p}+\mathbf{p}_{\ell}\right) \cdot \tau\left(\mathbf{p}_{\ell}\right)=0$ which implies $\mathbf{a}(\mathbf{p})-\mathbf{p}+\mathbf{p}_{\ell}=\mathbf{p}_{\ell}$ or $\mathbf{a}(\mathbf{p})-\mathbf{p}+\mathbf{p}_{\ell}=\mathbf{a}\left(\mathbf{p}_{\ell}\right)$. The first equation is impossible since $\mathbf{a}(\mathbf{p}) \neq \mathbf{p}$. The second yields, by Lemma $2.1, \mathbf{p}_{\ell}=\mathbf{p}$. Let $C=\left\{\ell \in\{2, \ldots, n+1\}: \mathbf{p}_{\ell}=\mathbf{p}\right\}$. For any $k \in C, g_{1 k} \cdot \tau_{1}=g(\mathbf{a}(\mathbf{p})) \cdot \tau(\mathbf{a}(\mathbf{p}))=0$, therefore row $k-1$ vanishes for all $k \in C$. If $k \notin C$, $g_{1 k} \cdot \tau_{k} \neq 0$, so statement $(i i)$ of the Lemma holds.

Case (2): there is $C \subset\{2, \ldots, n+1\},|C| \geq 2$, such that for all $k \in C, g_{1 k} \cdot \tau_{k}=0$. This forces $\mathbf{p}_{1}-\mathbf{p}+\mathbf{p}_{k} \in\left\{\mathbf{p}_{k}, \mathbf{a}\left(\mathbf{p}_{k}\right)\right\}$. Hence either $\mathbf{p}_{1}=\mathbf{p}$ or, by Lemma 2.1, $\mathbf{p}_{1}=\mathbf{a}(\mathbf{p})$ and $\mathbf{p}_{k}=\mathbf{p}$ for all $k \in C$. The remaining statements about the columns and rows of $\Gamma^{(n)}$ follow as in case (1).

In case $(i)$, only the first column of $\Gamma^{(n)}$ is nonzero. The right hand side is identical to the first column of $\Gamma^{(n)}$. In case (ii), vanishing of row number $k$ means $\mathbf{p}_{k}=\mathbf{p}$, so $g_{1 k} \cdot \tau=0$ at those points is obvious.

The last statement of the Lemma means that the system of equations remains consistent at the critical points because the right hand side remains in the span of the columns of $\Gamma^{(n)}$.

The condition that all momenta are on the Fermi surface can again be relaxed to a condition that all momenta be in a scale-independent neighbourhood of $S$, i.e. $|\rho|=|e(\mathbf{p})| \leq r_{\mathrm{o}}$, by the following Lemma. 
Lemma 4.4 For any $\varepsilon>0$, there are $r_{\mathrm{o}}>0$ and $s>0$, depending on $|e|_{2}$ and $u_{\mathrm{o}}$, such that if $|e(\mathbf{p})|<r_{\mathrm{o}}$ in all propagators and if $\left|\theta-\theta^{(0)}\right|<s$, then all critical points of $\Gamma^{(n)}$ are in an $\varepsilon$-neighbourhood of the critical points of $\rho=0, \theta=\theta^{(0)}$ given in Lemma 4.3.

Proof: Again, we order the proof according to the cases (1) and (2) of Remark 4.2. As mentioned, the critical point condition in case (1) is just a relabelling of that of the second-order case. Therefore, the critical points for $\theta_{1}$ and $\theta_{k}$ are $C^{1}$ in $\theta$ and the statement of the Lemma follows immediately from Lemma 3.4. If $\mathbf{p}_{\mathbf{1}}=\mathbf{p}$, that's it since the other momenta are not fixed in $(i)$ of Lemma 4.3. Let $\mathbf{p}_{\mathbf{1}}$ be near to $\mathbf{a}(\mathbf{p})$, and $\theta$ near to $\theta^{(0)}$. For $\ell \in\{2, \ldots, n+1\} \backslash\{k\}$, we perform a Taylor expansion of $g_{1 \ell} \cdot \tau_{\ell}$ in $\rho_{1}, \rho_{\ell}, \theta-\theta^{(0)}$, and $\theta_{1}-a\left(\theta^{(0)}\right)$, to obtain

$$
\begin{gathered}
\left|\nabla e\left(\mathbf{p}\left(\rho_{1}, \theta_{1}\right)-\mathbf{p}(0, \theta)+\mathbf{p}\left(\rho_{\ell}, \theta_{\ell}\right)\right) \cdot \partial_{\theta} \mathbf{p}\left(\rho_{\ell}, \theta_{\ell}\right)-\nabla e\left(\mathbf{p}\left(0, a\left(\theta^{(0)}\right)\right)-\mathbf{p}\left(0, \theta^{(0)}\right)+\mathbf{p}\left(0, \theta_{\ell}\right)\right) \cdot \partial_{\theta} \mathbf{p}\left(0, \theta_{\ell}\right)\right| \\
\leq \mathrm{const}\left(\left|\rho_{\ell}\right|+\left|\rho_{1}\right|+\left|\theta-\theta^{(0)}\right|+\left|\theta_{1}-a\left(\theta^{(0)}\right)\right|\right)
\end{gathered}
$$

where the constant is a bound for the Taylor remainder. It depends on $|e|_{2}$ and $u_{\mathrm{o}}$ because the Taylor remainder contains the second derivative of $e$ and objects like $\partial_{\rho} \mathbf{p}$ and $\partial_{\theta} \mathbf{p}$. It follows that for $r_{\mathrm{o}}$ and $\theta-\theta^{(0)}$ small enough, $\left|g_{1 \ell} \cdot \tau_{\ell}\right| \geq g_{1}>0$ unless $\theta_{\ell}$ is in an $\varepsilon$-neighbourhood of $\theta^{(0)}$.

For case (2), we do a similar Taylor expansion argument, but now applied to

$$
\left.\nabla e\left(\mathbf{p}\left(\rho_{1}, \theta_{1}\right)-\mathbf{p}(0, \theta)+\mathbf{p}\left(\rho_{k}, \theta_{k}\right)\right) \cdot \partial_{\theta} \mathbf{p}\left(\rho_{k}, \theta_{k}\right)\right)=0
$$

to get

$$
\nabla e\left(\mathbf{p}\left(0, \theta_{1}\right)-\mathbf{p}(0, \theta)+\mathbf{p}\left(0, \theta_{k}\right)\right) \cdot \partial_{\theta} \mathbf{p}\left(0, \theta_{k}\right)+\rho_{1} \phi_{1}+\rho_{k} \phi_{k}=0
$$

with $\phi_{i}$ continuous functions coming from Taylor expansion. By the Lemma 4.3, the first term in the sum only vanishes when $\theta=\theta_{1}$, or if $\theta=a\left(\theta_{1}\right)$ and $\theta_{k}=\theta$. Away from these points, it is nonzero and therefore for small enough $\left|\rho_{1}\right|$ and $\left|\rho_{k}\right|$, all critical points are in a neighbourhood of $\theta^{(0)}$.

Lemma 4.5 Let $k \geq 2, d=2$, and assume $(\mathrm{H} 1)_{k, 0},(\mathrm{H} 2)_{k, 0}$, and (H3)-(H5). Then the contribution of the particle-hole wicked ladder to the counterterm function $K$ is $C^{k}$.

Proof: The strategy to control the derivative is now similar to that of the second-order case: in the region away from the critical points, we do a change of variables that makes all propagators independent of the external momentum. Near the critical points, we show that every increase in the power of the denominator is accompanied by a small factor in the numerator. Again as before, we take one derivative with respect to the external momentum right away, so that $k-1$ derivatives remain to be taken after the manipulations.

Fix $\theta^{(0)}$, and let $\theta$ be in a sufficiently small neighbourhood of $\theta^{(0)}$. By Lemma 4.3 , any critical point of $\Gamma^{(n)}$ at $\theta$ is near to one of $\Gamma^{(n)}$ at $\theta^{(0)}$. Thus we may define the regular region dependent only on $\theta^{(0)}$. For $\delta>0$ and $a>0$, the regular region is defined as

$$
\mathcal{R}_{\delta}\left(\theta^{(0)}, a\right)=\left\{\Theta=\left(\theta_{1}, \ldots, \theta_{n+1}\right): \forall \Theta^{\prime} \text { with }\left|\Theta^{\prime}-\Theta\right| \leq \delta, \text { and } \forall\left|\theta-\theta^{(0)}\right| \leq a, \operatorname{rank} \Gamma^{(n)}(\rho, \Theta, \theta)=n\right\}
$$


Here we wrote $\Gamma^{(n)}(\rho, \Theta, \theta)$ to indicate that now the momenta on the lines of the graph do not have to be exactly on the Fermi surface, but they only have to be near it. By Lemma 4.4, the complement of the regular region is a neighbourhood of the critical points of $\Gamma^{(n)}=\Gamma^{(n)}(0, \Theta, \theta)$ given in Lemma 4.3. For $k \in\{1, \ldots, n+1\}$, let $\Gamma_{k}^{(n)}$ be the $n \times n$ submatrix of $\Gamma^{(n)}$ obtained by deleting column number $k$. We cover $\mathcal{R}_{\delta}$ with patches chosen such that in every patch, there is a $k \in\{1, \ldots, n+1\}$ such that $\operatorname{rank} \Gamma_{k}^{(n)}=n$. Since $\mathcal{R}_{\delta}$ is compact, the covering can be chosen to contain only finitely many such patches. Let $P$ be one of these patches and $\operatorname{rank} \Gamma_{k}^{(n)}(\Theta, \theta)=n$ for all $\Theta \in P$. Then $\Gamma_{k}^{(n)}$ is invertible, since it is a square matrix of maximal rank and the inverse is $C^{k-1}$ in all variables. Delete $\tilde{\theta}_{k}$ from the list of variables, and write $\tilde{\Theta}^{(k)}=\left(\tilde{\theta}_{1}, \ldots, \tilde{\theta}_{k-1}, \tilde{\theta}_{k+1}, \ldots, \tilde{\theta}_{n+1}\right)^{t}$. The change of variables is then given by by the solution to the initial value problem

$$
\begin{aligned}
\frac{\partial \tilde{\Theta}^{(k)}}{\partial \theta} & =\Gamma_{k}^{(n)}\left(\tilde{\Theta}^{(k)}, \theta\right)^{-1} \cdot \gamma\left(\tilde{\Theta}^{(k)}, \theta\right) \\
\tilde{\Theta}^{(k)}\left(\Theta^{(k)}, \theta^{(0)}\right) & =\Theta^{(k)} .
\end{aligned}
$$

The solution is a $C^{k}$ function of all variables because the right side of the differential equation is $C^{k-1}$. Thus the Jacobian is $C^{k-1}$ in all variables. After this change of variables, all the dependence on the external angle $\theta$ is in the Jacobian and in the support function of the patch. The patch itself is independent of $\theta$ since by construction it depends only on $\theta^{(0)}$. Thus, taking all the remaining derivatives is harmless. Moreover, the support restriction on the propagators reduces the integration regions for the $\tilde{\theta}_{k}$ in a way that cancels the large factor from the first derivative we took.

It remains to bound the contribution from the neighbourhood of the set where the rank of $\Gamma^{(n)}$ is nonmaximal. We split the proof in the two cases, according to the characterization of critical points given in Lemma 4.3.

(i) When $\mathbf{p}_{1}=\mathbf{p}$, only the first column in $\Gamma^{(n)}$ is nonzero. So, when $\mathbf{p}_{\mathbf{1}} \approx \mathbf{p}$, instead of a change of variables, we shall use that the derivative on a propagator on scale $j<0$ produces a small factor $M^{j}$ that cancels the extra big factor $M^{-j}$ from the denominator. We can be brief about this because the argument is very similar to that of the second order case. By Taylor expansion in the $\rho$ variables, we can reduce the problem to $\rho_{1}=\rho_{k}=0$. Let $\mathbf{p}_{1}$ be near to $\mathbf{p}$, and $\mathbf{p}_{2}, \ldots \mathbf{p}_{n+1}$ be arbitrary. Since $\mathbf{p}_{1} \approx \mathbf{p}$, we change variables from $\theta_{1}$ to $t=\theta_{1}-\theta$. Since $e\left(\mathbf{p}\left(\theta_{k}\right)\right)=0$, the usual Taylor expansion yields

$$
\begin{aligned}
e\left(\mathbf{p}\left(0, \theta_{1}\right)-\mathbf{p}(0, \theta)+\mathbf{p}\left(\theta_{k}\right)\right) & =t \phi\left(\theta, t, \theta_{k}\right) \\
\phi\left(\theta, t, \theta_{k}\right) & =\int_{0}^{1} d \alpha g\left(\mathbf{p}(\theta+\alpha t)-\mathbf{p}(\theta)+\mathbf{p}\left(\theta_{k}\right)\right) \cdot \tau(\mathbf{p}(\theta+\alpha t))
\end{aligned}
$$

If $\mathbf{p}_{k}$ is not in a small neighbourhood of $\mathbf{p}\left(\theta^{(0)}\right)$ or of $\mathbf{p}\left(a\left(\theta^{(0)}\right)\right)$, then $\phi\left(\theta, 0, \theta_{k}\right) \neq 0$, and therefore for all $|t|<\delta^{\prime}$ ( $\delta^{\prime}$ independent of the scales), $\left|\phi\left(\theta, t, \theta_{k}\right)\right| \geq \underline{\phi}>0$, so $\left|e\left(\mathbf{p}_{1}-\mathbf{p}+\mathbf{p}_{k}\right)\right| \leq M^{j}$ implies

$$
|t| \leq \text { const } M^{j}
$$

Thus, as in the second order case, every derivative acting on $C_{j}$ produces not only a large factor $M^{-j}$, but also a small factor $|t|$ that cancels it, e.g.

$$
\left|\frac{\partial}{\partial \theta} C_{j}\left(p_{0}, e\left(\mathbf{p}(\theta+t)-\mathbf{p}(\theta)+\mathbf{p}\left(\theta_{k}\right)\right)\right)\right| \leq|t|\left|\frac{\partial \phi}{\partial \theta}\right|\left|\partial_{2} C_{j}\right| \leq \text { const } M^{j} M^{-2 j}
$$


does not change the scaling behaviour of the propagator. The factor $M^{j}$ that got lost when the very first derivative was taken is regained, up to a factor $|j|$, by the volume improvement in the integration over $t$ coming from the restriction (4.14).

For any $k \geq 2$ for which $\theta_{k}$ is near to $\theta^{(0)}$ (and hence near to $\theta$ ), change variables from $\theta_{k}$ to $y=\theta_{k}-\theta$. Then the $\theta$-dependence in the propagator is in the function

$$
\eta(\theta, x, y)=e(\mathbf{p}(\theta+x)+\mathbf{p}(\theta+y)-\mathbf{p}(\theta))
$$

The analysis of the singularity is now identical to that to the second-order case, and in particular, Lemma 3.3 applies. Thus for every increase in the power of the denominator, there is a corresponding small factor in the numerator. The details of the bound are as in the second-order case.

For any $k \geq 2$ for which $\theta_{k}$ is near to $a\left(\theta^{(0)}\right)$, hence near to $a(\theta)$, change variables from $\theta_{k}$ to $y=\theta_{k}-a(\theta)$. Then the $\theta$-dependence in the propagator is in the function

$$
\eta(\theta, x, y)=e(\mathbf{p}(\theta+x)+\mathbf{p}(a(\theta)+y)-\mathbf{p}(\theta))
$$

The analysis of the singularity is now identical to the second-order case, in particular, Lemma 3.3 applies. All the details are as in the second-order case.

There remains the case $(i i): \mathbf{p}_{1}=a(\mathbf{p})$, and $\mathbf{p}_{k}=\mathbf{p}$ for $k \in C \subset\{2, \ldots, n+1\}$. We change variables from $\theta_{1}$ to $\tilde{\theta}_{1}=\theta_{1}-a(\theta)$. Then $\frac{\partial \tilde{\theta}_{1}}{\partial \theta_{1}}=1$, and the first column of $\Gamma^{(n)}$ is moved to the right hand side of the equation. For $k \in C$, we change variables from $\theta_{k}$ to $\tilde{\theta}_{k}=\theta_{k}-\theta$ and move the corresponding columns of $\Gamma^{(n)}$ to the right hand side of the equation. We delete row number $k$ for every $k \in C$. The square matrix left over of $\Gamma^{(n)}$ after this procedure has maximal rank by construction and Lemma 4.3, so the change of variables is there as in the regular region. We solve the resulting system of differential equations for $\tilde{\theta}_{k}, k \notin C$. For $k \in C$,

$$
e\left(\mathbf{p}_{1}-\mathbf{p}+\mathbf{p}_{k}\right)=e(\mathbf{p}(a(\theta)+x)-\mathbf{p}(\theta)+\mathbf{p}(\theta+y))
$$

so Lemma 3.3 applies.

We now turn to the particle-particle wicked ladder (see Figure $6(b)$ ). The analogue of Lemma 4.3 is in the symmetric case

Lemma 4.6 Assume (Sy). For the particle-particle wicked ladder, if rank $\Gamma^{(n)} \leq n-1$ and all momenta are on $S$ then one of the following holds.

(i) $\quad \mathbf{p}_{\mathbf{1}}=-\mathbf{p}$; then rank $\Gamma^{(n)} \leq 1$ and all columns of $\Gamma^{(n)}$ except for the first vanish.

(ii) $\mathbf{p}_{1}=\mathbf{p}$, and there is $\emptyset \neq C \subset\{2, \ldots, n+1\}$ such that for all $k \in C, \mathbf{p}_{k}=\mathbf{p}$, and for all $l \notin C$, $\mathbf{p}_{l} \neq \mathbf{p}$; then rank $\Gamma^{(n)}=n-|C|$, and

all columns with index $k \in C$ vanish: $\Gamma_{m k}^{(n)}=0$ for all $m \in\{2, \ldots, n+1\}$,

all rows with index $k-1, k \in C$, vanish: $\Gamma_{k-1, m}^{(n)}=0$ for all $m \in\{1, \ldots, n\}$,

all columns with index $k \in\{2, \ldots, n+1\} \backslash C$ are nonzero.

Whenever row $k$ of $\Gamma^{(n)}$ vanishes, the corresponding component $\gamma_{k}$ of the vector $\gamma$ on the right hand side of (4.6) also vanishes. In case (i), the right hand side $\gamma$ coincides with the first column of $\Gamma^{(n)}$. 
Proof: To start, we do not assume (Sy). Case (1) is as in second order, so $\mathbf{p}_{\mathbf{1}}=\mathbf{p}_{k}=\mathbf{p}$ or $\mathbf{p}_{\mathbf{1}}=\mathbf{a}(\mathbf{p})$, $\mathbf{p}_{k}=\mathbf{p}$, or $\mathbf{p}_{1}=\mathbf{p}_{k}=\mathbf{a}(\mathbf{p})$. In case $(2)$, the condition that $g_{1 k} \cdot \tau_{k}=0$ for $k \in C,|C| \geq 2$, forces $\mathbf{p}_{\mathbf{1}}+\mathbf{p}-\mathbf{p}_{k} \in\left\{\mathbf{p}_{k}, \mathbf{a}\left(\mathbf{p}_{k}\right)\right\}$ for all $k \in C$. This is possible only if either $\mathbf{p}_{\mathbf{1}}=\mathbf{p}, \mathbf{p}_{k}=\mathbf{p}$ for all $k \in C$, or if

$$
\mathbf{p}_{1}+\mathbf{p}=\mathbf{p}_{k}+\mathbf{a}\left(\mathbf{p}_{k}\right) \quad \text { for all } k \in C .
$$

Note that in the asymmetric case it does not follow from (4.19) that $\mathbf{p}_{k}$ or $\mathbf{p}_{\mathbf{1}}$ have to be $\mathbf{p}$ or $\mathbf{a}(\mathbf{p})$ : although there is the solution $\mathbf{p}_{\mathbf{1}}=\mathbf{p}_{k}, \mathbf{p}=\mathbf{a}\left(\mathbf{p}_{k}\right)$, there can be other solutions, due to the asymmetry of $S$. At these additional solutions, the system of equations (4.6) is inconsistent.

We now assume (Sy). Then $\mathbf{a}\left(\mathbf{p}_{k}\right)=-\mathbf{p}_{k}$, and (4.19) implies $\mathbf{p}_{\mathbf{1}}=-\mathbf{p}$. Looking back at all cases, we see that they are covered by statements $(i)$ and $(i i)$ of the Lemma. The statements about the rank and the vanishing of columns and rows of $\Gamma^{(n)}$, and about the consistency at the critical points follow by inspection of the matrix.

Lemma 4.7 Let $k \geq 2, d=2$, and assume $(\mathrm{H} 1)_{k, 0},(\mathrm{H} 2)_{k, 0},(\mathrm{H} 3)-(\mathrm{H} 5)$, and (Sy). Then the contribution of the particle-particle wicked ladder to the counterterm function $K$ is $C^{k}$.

The proof of this Lemma, as well as that of the analogue of Lemma 4.4, is an obvious variation of that of Lemma 4.5, and we leave it to the reader. We instead turn to the nonsymmetric case in which the potential inconsistencies of (4.6) at solutions of (4.19) caused problems. The main point there is the same that leads to the Fermi liquid behaviour of such models: The particle-particle bubble, which in the symmetric case prevents the convergence of perturbation theory because of its singularity at zero transfer momentum, has no singularities. Under our assumptions, this can be deduced from the following Lemma.

Lemma 4.8 Let $d=2, h>0$, and $(\mathrm{H} 2)_{2, h}-\left(\mathrm{H} 4^{\prime}\right)$ hold and assume that (Sy) does not hold. Then there is a constant $Q_{B}>0$ such that for all $\varepsilon_{2} \geq \varepsilon_{1}>0$ and for all $\mathbf{q} \in \mathcal{B}$

$$
\left.\sup _{|\rho| \leq \varepsilon_{1}} \int d \theta \mathbb{1}(\mid e(-\mathbf{p}(\rho, \theta))+\mathbf{q}) \mid \leq \varepsilon_{2}\right) \leq Q_{B} \varepsilon_{2}{ }^{\frac{1}{3}}
$$

and therefore

$$
\int_{\mathcal{B}} d^{d} \mathbf{p} \mathbb{1}\left(|e(-\mathbf{p}+\mathbf{q})| \leq \varepsilon_{2}\right) \quad \mathbb{1}\left(|e(\mathbf{p})| \leq \varepsilon_{1}\right) \leq 2|J|_{\mathrm{o}} Q_{B} \varepsilon_{1} \varepsilon_{2}{ }^{\frac{1}{3}}
$$

where $J$ is the Jacobian defined in (2.15).

Proof: See Appendix C.

It is important in the proof that the curvature of $S$ at $\mathbf{p}$ and $\mathbf{a}(\mathbf{p})$ differs except at a finite number of points; see Appendix C. Given Lemma 4.8, the boundedness of the particle-particle bubble is obvious because instead of the ordinary power counting behaviour $M^{0 j}$ of a non-overlapping four-legged diagram, Lemma 4.8 implies a bound by $M^{j / 3}$ which gives a convergent scale sum. Similarly, the proof that the particleparticle wicked ladder is $C^{2}$ is now an easy consequence of volume estimates; it does not require any analysis 
of critical points. In particular, assumption (H5) is not needed because it was needed neither in the proof of Theorem 1.1 nor in that of Lemma 4.8.

Theorem 4.9 Let $d \geq 2, h>0$, and $(\mathrm{H} 1)_{2, h},(\mathrm{H} 2)_{2, h}$, and (H3)-(H4') hold, and assume that (Sy) does not hold. Let $G$ be the particle-particle wicked ladder shown in Figure $4(b)$, and denote $\operatorname{Val}(G)(\mathbf{p}(\rho, \theta))$, defined in (4.2), by $R_{j}(\rho, \theta)$. Then the scale sum $\sum_{j<0} R_{j}(\rho, \theta)$ is $C^{2}$ both in $\rho$ and $\theta$.

Proof: The idea of the proof is simple: by Lemma 4.8 there is a volume gain in any of the bubbles uniformly in the transfer momentum. There are enough loops to extract both that gain in one bubble and the volume gain from two overlapping loops. This gives an improvement factor $M^{4 j / 3}$, hence enough decay to take two derivatives, no matter whether they are taken in $\rho$ or in $\theta$ direction.

To do the details, it is most convenient to choose the spanning tree for $G$ as shown in Figure 7 because then the derivative acts only on the propagator of line $\ell_{1}$, and we avoid some uninteresting combinatorics (in Figure 7, the additional bubbles are all put into a subgraph drawn as the shaded disk). Taking two derivatives, using (2.54), and doing the $p_{0}$-integrals in the usual way, we get (denoting $\mathbb{1}_{j}(e(\mathbf{p}))=\mathbb{1}\left(|e(\mathbf{p})| \leq M^{j}\right)$ )

$$
\left|D^{2} R_{j}(\rho, \theta)\right|=W_{2} W_{\mathrm{o}}^{2 n} M^{-2 n j} M^{-3 j}\left(2 M^{j}\right)^{n+1} \int \prod_{l \notin L(T)} d^{d} \mathbf{p}_{l} \prod_{l \in L(G)} \mathbb{1}_{j}\left(e\left(\mathbf{p}_{l}\right)\right)
$$

where the $M^{-2 n j}$ comes from the sup norm of the propagators in the bubbles, the $M^{-3 j}$ comes from the second derivative of the propagator on line $\ell_{1}$, and the factor $\left(2 M^{j}\right)^{n+1}$ comes from the $p_{0}$-integrations. We call the $n+1$ loop variables $\mathbf{p}_{1}, \ldots, \mathbf{p}_{n+1}$, and introduce $\left(\rho_{l}, \theta_{l}\right)$ as integration variables such that $\mathbf{p}_{k}=\mathbf{p}\left(\rho_{k}, \theta_{k}\right)$, and do the $\rho$-integrations. Every $\rho_{k}$ produces a factor const $M^{j}$ when integrated. Thus

$$
\left|R_{j}\right|_{2} \leq \nu M^{-j} \sup _{\substack{\rho_{1}, \ldots, \rho_{n}+1 \\\left|\rho_{k}\right| \leq M^{j}}} \int \prod_{i=1}^{n+1} d \theta_{i} \prod_{\ell \in L(T)} \mathbb{1}_{j}\left(e\left(\mathbf{q}_{\ell}\right)\right)
$$

where the momenta $\mathbf{q}_{\ell}$ for $\ell \in L(T)$ are given as linear combinations of the loop momenta $\mathbf{p}\left(\rho_{1}, \theta_{1}\right), \ldots$, $\mathbf{p}\left(\rho_{n+1}, \theta_{n+1}\right)$, and the external momentum $\mathbf{p}(\rho, \theta)$, and where $\nu$ contains all the constants. We may choose the labelling of the loop momenta as indicated in Figure 7. Since $n \geq 2$, i.e. there are at least two bubbles, so the product over $\ell \in L(T)$ contains at least the two factors $\mathbb{1}_{j}\left(e\left(\mathbf{q}_{\ell_{1}}\right)\right)$ and $\mathbb{1}_{j}\left(e\left(\mathbf{q}_{\ell_{2}}\right)\right)$, where $\ell_{1}$ and $\ell_{2}$ are as in Figure 7. $\mathbf{q}_{\ell_{1}}=\mathbf{p}_{1}+\mathbf{p}_{2}-\mathbf{p}$ and $\mathbf{q}_{\ell_{2}}=\mathbf{p}_{1}+\mathbf{p}_{2}-\mathbf{p}_{3}$, so

$$
\left|R_{j}\right|_{2} \leq \operatorname{const} M^{-j} \sup _{\left|\rho_{1}\right| \leq M^{j},\left|\rho_{2}\right| \leq M^{j}} \int d \theta_{1} d \theta_{2} \mathbb{1}_{j}\left(e\left(\mathbf{p}_{1}+\mathbf{p}_{2}-\mathbf{p}\right)\right) \sup _{\left|\rho_{3}\right| \leq M^{j}} \int d \theta_{3} \mathbb{1}_{j}\left(e\left(-\mathbf{p}_{3}+\mathbf{p}_{1}+\mathbf{p}_{2}\right)\right) .
$$

By Lemma 4.8, the integral over $\theta_{3}$ is bounded uniformly in $\mathbf{p}_{1}$ and $\mathbf{p}_{2}$, and by a Taylor expansion in $\rho_{1}$ and $\rho_{2}$, as used to derive (3.24), we get

$$
\left|R_{j}\right|_{2} \leq \text { const } M^{-j} Q_{B} M^{j / 3} \mathcal{W}\left(\left(1+2 \frac{|e|_{1}}{u_{o}}\right) M^{j}\right) \leq \text { const }|j| M^{j / 3}
$$

so that

$$
\sum_{j<0}\left|R_{j}(\rho, \theta)\right|_{2} \leq \text { const } \sum_{j<0}|j| M^{j / 3} \leq \text { const } \frac{1}{1-M^{-1 / 6}}
$$




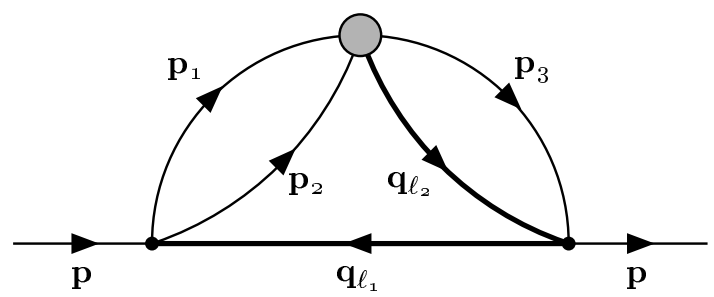

Figure 7

Remark 4.10 The reason why the problematic critical points of (4.19) did not appear in second order is simply that in second order, the case (2) mentioned before Lemma 4.3 cannot occur because there are only two loop momenta. The conditions of case (1) exclude (4.19).

Remark 4.11 For $d \geq 3$, the proof is similar to that of Theorem 3.10 because, again, one has restrictions to a neighbourhood of the critical points. Lemma 3.9 applies directly to do the argument. 


\section{Appendix A. The $C^{2}$ Morse Lemmas}

In this Appendix, we prove the $C^{2}$ Morse Lemmas that we need to prove Theorem 1.1. The proof of the Morse Lemma for smooth functions is in many textbooks, but our functions are only $C^{2}$, which makes the proof less straightforward. One proof of the $C^{2}$ Morse lemma can be found in [MW]. For convenience of the reader, we include another proof here.

\section{A.1 Hyperbolic case}

Let $\nu\left(\phi_{1}, \phi_{2}\right)$ be a $C^{2}$ function in a neighbourhood of $(0,0)$ obeying

$$
\begin{aligned}
& \nu(0,0)=0 \\
& \partial_{1} \nu(0,0)=0 \quad \partial_{1} \nu(0,0) \quad=0 \\
& \partial_{1}^{2} \nu(0,0)=-1 \quad \partial_{2}^{2} \nu(0,0)=1 \quad \partial_{1} \partial_{2} \nu(0,0)=0
\end{aligned}
$$

Lemma A.1 There exists a neighbourhood $\mathcal{N}$ of 0 and two $C^{1}$ functions $\psi_{ \pm}\left(\phi_{1}\right)$ such that

$$
\nu\left(\phi_{1}, \psi_{ \pm}\left(\phi_{1}\right)\right)=0 \quad \text { for all } \phi_{1} \in \mathcal{N}
$$

and

$$
\begin{aligned}
& \psi_{ \pm}(0)=0 \\
& \psi_{ \pm}^{\prime}(0)= \pm 1
\end{aligned}
$$

Theorem A.2 There exist $C^{1}$ functions $x\left(\phi_{1}, \phi_{2}\right)$ and $y\left(\phi_{1}, \phi_{2}\right)$ in a neighbourhood of $(0,0)$ such that

$$
\nu\left(\phi_{1}, \phi_{2}\right)=x\left(\phi_{1}, \phi_{2}\right) y\left(\phi_{1}, \phi_{2}\right)
$$

and

$$
\begin{aligned}
& x(0,0)=0 \quad y(0,0) \quad=0 \\
& \partial_{1} x(0,0)=1 \quad \partial_{2} x(0,0) \quad=-1 \\
& \partial_{1} y(0,0)=-1 / 2 \quad \partial_{2} y(0,0) \quad=1 / 2
\end{aligned}
$$

The proofs of both Lemma A.1 and Theorem A.2 use the representations

$$
\nu\left(\phi_{1}, \phi_{2}\right)=-\frac{1}{2} \alpha\left(\phi_{1}, \phi_{2}\right) \phi_{1}^{2}+\beta\left(\phi_{1}, \phi_{2}\right) \phi_{1} \phi_{2}+\frac{1}{2} \gamma\left(\phi_{1}, \phi_{2}\right) \phi_{2}^{2}
$$


and

$$
\begin{aligned}
& \partial_{1} \nu\left(\phi_{1}, \phi_{2}\right)=-\tilde{\alpha}\left(\phi_{1}, \phi_{2}\right) \phi_{1}+\tilde{\beta}\left(\phi_{1}, \phi_{2}\right) \phi_{2} \\
& \partial_{2} \nu\left(\phi_{1}, \phi_{2}\right)=\tilde{\beta}\left(\phi_{1}, \phi_{2}\right) \phi_{1}+\tilde{\gamma}\left(\phi_{1}, \phi_{2}\right) \phi_{2}
\end{aligned}
$$

where all the coefficients on the right hand side are continuous and where

$$
\begin{aligned}
& \alpha(0,0)=\tilde{\alpha}(0,0)=1 \\
& \gamma(0,0)=\tilde{\gamma}(0,0)=1 \\
& \beta(0,0)=\tilde{\beta}(0,0)=0
\end{aligned}
$$

We shall use the notation $o(1)$ to denote a generic and unimportant continuous function that vanishes at $(0,0)$. Hence, for example, we shall write $\alpha\left(\phi_{1}, \phi_{2}\right)=1+o(1)$. To prove $(A .6)$ one uses

$$
\begin{aligned}
\nu\left(\phi_{1}, \phi_{2}\right) & =\int_{0}^{1} d t(1-t) \frac{d^{2}}{d t^{2}} \nu\left(t \phi_{1}, t \phi_{2}\right) \\
& =\int_{0}^{1} d t(1-t)\left[\partial_{1}^{2} \nu\left(t \phi_{1}, t \phi_{2}\right) \phi_{1}^{2}+2 \partial_{1} \partial_{2} \nu\left(t \phi_{1}, t \phi_{2}\right) \phi_{1} \phi_{2}+\partial_{2}^{2} \nu\left(t \phi_{1}, t \phi_{2}\right) \phi_{2}^{2}\right]
\end{aligned}
$$

Hence

$$
\begin{aligned}
& \alpha\left(\phi_{1}, \phi_{2}\right)=-2 \int_{0}^{1} d t(1-t) \partial_{1}^{2} \nu\left(t \phi_{1}, t \phi_{2}\right) \\
& \beta\left(\phi_{1}, \phi_{2}\right)=2 \int_{0}^{1} d t(1-t) \partial_{1} \partial_{2} \nu\left(t \phi_{1}, t \phi_{2}\right) \\
& \gamma\left(\phi_{1}, \phi_{2}\right)=2 \int_{0}^{1} d t(1-t) \partial_{2}^{2} \nu\left(t \phi_{1}, t \phi_{2}\right)
\end{aligned}
$$

Similarly, to prove $(A .7)$ one uses

$$
\begin{aligned}
\partial_{1} \nu\left(\phi_{1}, \phi_{2}\right) & =\int_{0}^{1} d t \frac{d}{d t} \partial_{1} \nu\left(t \phi_{1}, t \phi_{2}\right) \\
& =\int_{0}^{1} d t\left[\partial_{1}^{2} \nu\left(t \phi_{1}, t \phi_{2}\right) \phi_{1}+\partial_{1} \partial_{2} \nu\left(t \phi_{1}, t \phi_{2}\right) \phi_{2}\right] \\
\partial_{2} \nu\left(\phi_{1}, \phi_{2}\right) & =\int_{0}^{1} d t \frac{d}{d t} \partial_{2} \nu\left(t \phi_{1}, t \phi_{2}\right) \\
& =\int_{0}^{1} d t\left[\partial_{1} \partial_{2} \nu\left(t \phi_{1}, t \phi_{2}\right) \phi_{1}+\partial_{2}^{2} \nu\left(t \phi_{1}, t \phi_{2}\right) \phi_{2}\right]
\end{aligned}
$$

to yield

$$
\begin{aligned}
& \tilde{\alpha}\left(\phi_{1}, \phi_{2}\right)=-\int_{0}^{1} d t \partial_{1}^{2} \nu\left(t \phi_{1}, t \phi_{2}\right) \\
& \tilde{\beta}\left(\phi_{1}, \phi_{2}\right)=\int_{0}^{1} d t \partial_{1} \partial_{2} \nu\left(t \phi_{1}, t \phi_{2}\right) \\
& \tilde{\gamma}\left(\phi_{1}, \phi_{2}\right)=\int_{0}^{1} d t \partial_{2}^{2} \nu\left(t \phi_{1}, t \phi_{2}\right)
\end{aligned}
$$

Proof of Lemma A.1: First fix any sufficiently small $\phi_{1}$. If $\alpha, \beta, \gamma$ were constants $\nu\left(\phi_{1}, \phi_{2}\right)=0$ would have two solutions, namely

$$
\phi_{2}=\frac{-\beta \phi_{1} \pm \sqrt{\beta^{2} \phi_{1}^{2}+\alpha \gamma \phi_{1}^{2}}}{\gamma}
$$


So define

$$
\Psi_{ \pm}\left(\phi_{1}, \phi_{2}\right)=\frac{\sqrt{\alpha\left(\phi_{1}, \phi_{2}\right) \gamma\left(\phi_{1}, \phi_{2}\right)+\beta^{2}\left(\phi_{1}, \phi_{2}\right)} \mp \beta\left(\phi_{1}, \phi_{2}\right)}{\gamma\left(\phi_{1}, \phi_{2}\right)}=1+o(1)
$$

We have

$$
\nu\left(\phi_{1}, \phi_{2}\right)=\frac{1}{2} \gamma\left(\phi_{1}, \phi_{2}\right)\left[\phi_{2}-\Psi_{+}\left(\phi_{1}, \phi_{2}\right) \phi_{1}\right]\left[\phi_{2}+\Psi_{-}\left(\phi_{1}, \phi_{2}\right) \phi_{1}\right]
$$

Note that the factor $\frac{1}{2} \gamma\left(\phi_{1}, \phi_{2}\right)$ never vanishes, the next factor obeys

$$
\begin{aligned}
& \phi_{2}-\Psi_{+}\left(\phi_{1}, \phi_{2}\right) \phi_{1}<0 \quad \text { if } \quad \phi_{2}<\phi_{1} \begin{cases}\min \Psi_{+} & \text {if } \phi_{1}>0 \\
\max \Psi_{+} & \text {if } \phi_{1}<0\end{cases} \\
& \phi_{2}-\Psi_{+}\left(\phi_{1}, \phi_{2}\right) \phi_{1}>0 \quad \text { if } \phi_{2}>\phi_{1} \begin{cases}\max \Psi_{+} & \text {if } \phi_{1}>0 \\
\min \Psi_{+} & \text {if } \phi_{1}<0\end{cases}
\end{aligned}
$$

and the last factor obeys

$$
\begin{aligned}
& \phi_{2}+\Psi_{-}\left(\phi_{1}, \phi_{2}\right) \phi_{1}>0 \quad \text { if } \phi_{2}>-\phi_{1} \begin{cases}\min \Psi_{-} & \text {if } \phi_{1}>0 \\
\max \Psi_{-} & \text {if } \phi_{1}<0\end{cases} \\
& \phi_{2}+\Psi_{-}\left(\phi_{1}, \phi_{2}\right) \phi_{1}<0 \quad \text { if } \phi_{2}<-\phi_{1} \begin{cases}\max \Psi_{-} & \text {if } \phi_{1}>0 \\
\min \Psi_{-} & \text {if } \phi_{1}<0\end{cases}
\end{aligned}
$$

If necessary, restrict the neighbourhood so that $\min \Psi_{ \pm}$and $\max \Psi_{ \pm}$all lie in $[1 / 2,3 / 2]$. Then, still for fixed $\phi_{1}, \nu\left(\phi_{1}, \phi_{2}\right)$ necessarily changes sign at least once between $\phi_{1} \min \Psi_{+}$and $\phi_{1} \max \Psi_{+}$and at least once between $-\phi_{1} \min \Psi_{-}$and $-\phi_{1} \max \Psi_{-}$. But in a neighbourhood of the origin $\partial_{2}^{2} \nu>0$, so that $\nu$, viewed as a function of $\phi_{2}$, is strictly convex and can have at most two zeroes. So it has exactly two zeroes, one in each of the aforementioned intervals. Call the zeroes $\psi_{ \pm}\left(\phi_{1}\right)$.

The zeroes obey

$$
\begin{aligned}
& \psi_{+}\left(\phi_{1}\right)=\Psi_{+}\left(\phi_{1}, \psi_{+}\left(\phi_{1}\right)\right) \phi_{1} \\
& \psi_{-}\left(\phi_{1}\right)=-\Psi_{-}\left(\phi_{1}, \psi_{-}\left(\phi_{1}\right)\right) \phi_{1},
\end{aligned}
$$

so

$$
\nu\left(\phi_{1}, \phi_{2}\right)=\frac{1}{2} \gamma\left(\phi_{1}, \phi_{2}\right)\left(\phi_{2}-\psi_{+}\left(\phi_{1}\right)\right)\left(\phi_{2}+\psi_{-}\left(\phi_{1}\right)\right) .
$$

Since, to be in the aforementioned intervals, $\left|\psi_{ \pm}\left(\phi_{1}\right)\right| \leq\left|\phi_{1}\right| \max \Psi_{ \pm}$and since $\Psi_{ \pm}=1+o(1)$, we have that $\psi_{ \pm}$is differentiable at $\phi_{1}=0$ and obeys

$$
\begin{aligned}
& \psi_{ \pm}(0)=0 \\
& \psi_{ \pm}^{\prime}(0)= \pm 1
\end{aligned}
$$

As $\nu\left(\phi_{1}, \psi_{ \pm}\left(\phi_{1}\right)\right)=0$ we have for all $\phi_{1} \neq 0$

$$
\psi_{ \pm}^{\prime}\left(\phi_{1}\right)=-\frac{\partial_{1} \nu\left(\phi_{1}, \psi_{ \pm}\left(\phi_{1}\right)\right)}{\partial_{2} \nu\left(\phi_{1}, \psi_{ \pm}\left(\phi_{1}\right)\right)}
$$

Note that for the denominator $\partial_{2} \nu\left(\phi_{1}, \psi_{ \pm}\left(\phi_{1}\right)\right)$ to vanish $\psi_{ \pm}(\phi)$ must be a double zero of $\nu\left(\phi_{1}, \cdot\right)$ and we know that this cannot happen for $\phi_{1} \neq 0$. Hence $\psi_{ \pm}$is $C^{1}$ away from $\phi_{1}=0$. It only remains to verify the continuity of $\psi_{ \pm}^{\prime}$ at $\phi_{1}=0$. From $(A .7)$

$$
\begin{aligned}
\psi_{ \pm}^{\prime}\left(\phi_{1}\right) & =-\frac{\partial_{1} \nu\left(\phi_{1}, \psi_{ \pm}\left(\phi_{1}\right)\right)}{\partial_{2} \nu\left(\phi_{1}, \psi_{ \pm}\left(\phi_{1}\right)\right)} \\
& =-\frac{-\tilde{\alpha}\left(\phi_{1}, \psi_{ \pm}\left(\phi_{1}\right)\right) \phi_{1}+\tilde{\beta}\left(\phi_{1}, \psi_{ \pm}\left(\phi_{1}\right)\right) \psi_{ \pm}\left(\phi_{1}\right)}{\tilde{\beta}\left(\phi_{1}, \psi_{ \pm}\left(\phi_{1}\right)\right) \phi_{1}+\tilde{\gamma}\left(\phi_{1}, \psi_{ \pm}\left(\phi_{1}\right)\right) \psi_{ \pm}\left(\phi_{1}\right)} \\
& =-\frac{-[1+o(1)] \phi_{1} \pm o(1) \Psi_{ \pm}\left(\phi_{1}, \psi_{ \pm}\left(\phi_{1}\right)\right) \phi_{1}}{o(1) \phi_{1} \pm[1+o(1)] \Psi_{ \pm}\left(\phi_{1}, \psi_{ \pm}\left(\phi_{1}\right)\right) \phi_{1}} \\
& = \pm 1+o(1)
\end{aligned}
$$


Proof of Theorem A.2: Define

$$
\begin{aligned}
& x\left(\phi_{1}, \phi_{2}\right)=\phi_{2}-\psi_{+}\left(\phi_{1}\right) \\
& y\left(\phi_{1}, \phi_{2}\right)=\int_{0}^{1} d \alpha \partial_{2} \nu\left(\phi_{1},(1-\alpha) \psi_{+}\left(\phi_{1}\right)+\alpha \phi_{2}\right) .
\end{aligned}
$$

Then $\nu\left(\phi_{1}, \phi_{2}\right)=x\left(\phi_{1}, \phi_{2}\right) y\left(\phi_{1}, \phi_{2}\right)$ holds by Taylor expansion. That $x$ is $C^{1}$, that $x(0,0)=0$, that $\partial_{1} x(0,0)=-1$ and that $\partial_{2} x(0,0)=1$ are all obvious, and so are the statements for $y$.

\section{A.2 Elliptic case}

Let $\nu\left(\phi_{1}, \phi_{2}\right)$ be a $C^{2}$ function in a neighbourhood of $(0,0)$ obeying

$$
\begin{aligned}
& \nu(0,0)=0 \\
& \partial_{1} \nu(0,0)=0 \quad \partial_{1} \nu(0,0) \quad=0 \\
& \partial_{1}^{2} \nu(0,0)=1 \quad \partial_{2}^{2} \nu(0,0)=1 \quad \partial_{1} \partial_{2} \nu(0,0)=0
\end{aligned}
$$

Theorem A.3 Define $\theta=\tan ^{-1} \frac{\phi_{2}}{\phi_{2}}$ to be the usual polar angle and $R=\nu\left(\phi_{1}, \phi_{2}\right)$. Then $R(0,0)=0, R$ is increasing on each fixed ray $\theta=$ const and the Jacobian

$$
\frac{\partial(R, \theta)}{\partial\left(\phi_{1}, \phi_{2}\right)}=1+o(1)
$$

Define

$$
\begin{aligned}
& x\left(\phi_{1}, \phi_{2}\right)=\sqrt{2 R\left(\phi_{1}, \phi_{2}\right)} \cos \theta\left(\phi_{1}, \phi_{2}\right) \\
& y\left(\phi_{1}, \phi_{2}\right)=\sqrt{2 R\left(\phi_{1}, \phi_{2}\right)} \sin \theta\left(\phi_{1}, \phi_{2}\right)
\end{aligned}
$$

Then

$$
\nu\left(\phi_{1}, \phi_{2}\right)=\frac{1}{2}\left(x\left(\phi_{1}, \phi_{2}\right)^{2}+y\left(\phi_{1}, \phi_{2}\right)^{2}\right)
$$

and the Jacobian

$$
\frac{\partial(x, y)}{\partial\left(\phi_{1}, \phi_{2}\right)}=1+o(1)
$$

Remark A.4 $R$ plays the role of $\frac{1}{2} r^{2}$ in the usual polar coordinates. 
Proof: We now have the representations (with new notation)

$$
\begin{aligned}
\nu\left(\phi_{1}, \phi_{2}\right) & =\frac{1}{2} \alpha\left(\phi_{1}, \phi_{2}\right) \phi_{1}^{2}+\beta\left(\phi_{1}, \phi_{2}\right) \phi_{1} \phi_{2}+\frac{1}{2} \gamma\left(\phi_{1}, \phi_{2}\right) \phi_{2}^{2} \\
\partial_{1} \nu\left(\phi_{1}, \phi_{2}\right) & =\hat{\alpha}\left(\phi_{1}, \phi_{2}\right) \phi_{1}+\hat{\beta}\left(\phi_{1}, \phi_{2}\right) \phi_{2} \\
\partial_{2} \nu\left(\phi_{1}, \phi_{2}\right) & =\hat{\beta}\left(\phi_{1}, \phi_{2}\right) \phi_{1}+\hat{\gamma}\left(\phi_{1}, \phi_{2}\right) \phi_{2}
\end{aligned}
$$

where

$$
\begin{array}{rlrl}
\alpha\left(\phi_{1}, \phi_{2}\right) & =2 \int_{0}^{1} d t(1-t) \partial_{1}^{2} \nu\left(t \phi_{1}, t \phi_{2}\right) & =1+o(1) \\
\beta\left(\phi_{1}, \phi_{2}\right) & =2 \int_{0}^{1} d t(1-t) \partial_{1} \partial_{2} \nu\left(t \phi_{1}, t \phi_{2}\right) & =o(1) \\
\gamma\left(\phi_{1}, \phi_{2}\right) & =2 \int_{0}^{1} d t(1-t) \partial_{2}^{2} \nu\left(t \phi_{1}, t \phi_{2}\right) & & =1+o(1) \\
\hat{\alpha}\left(\phi_{1}, \phi_{2}\right) & =\int_{0}^{1} d t \partial_{1}^{2} \nu\left(t \phi_{1}, t \phi_{2}\right) & & =1+o(1) \\
\hat{\beta}\left(\phi_{1}, \phi_{2}\right) & =\int_{0}^{1} d t \partial_{1} \partial_{2} \nu\left(t \phi_{1}, t \phi_{2}\right) & & =o(1) \\
\hat{\gamma}\left(\phi_{1}, \phi_{2}\right) & =\int_{0}^{1} d t \partial_{2}^{2} \nu\left(t \phi_{1}, t \phi_{2}\right) & & =1+o(1)
\end{array}
$$

That $R(0,0)=0$ and $R$ is increasing on each fixed ray follows easily from

$$
\begin{aligned}
\nu\left(\phi_{1}, \phi_{2}\right) & =\frac{1}{2}\left(\phi_{1}^{2}+\phi_{2}^{2}\right)+o(1) \phi_{1}^{2}+o(1) \phi_{1} \phi_{2}+o(1) \phi_{2}^{2} \\
\phi_{1} \partial_{1} \nu\left(\phi_{1}, \phi_{2}\right)+\phi_{2} \partial_{2} \nu\left(\phi_{1}, \phi_{2}\right) & =\phi_{1}^{2}+\phi_{2}^{2}+o(1) \phi_{1}^{2}+o(1) \phi_{1} \phi_{2}+o(1) \phi_{2}^{2}
\end{aligned}
$$

The Jacobian

$$
\begin{aligned}
\left(\begin{array}{cc}
\frac{\partial R}{\partial \phi_{1}} & \frac{\partial R}{\partial \phi_{2}} \\
\frac{\partial \theta}{\partial \phi_{1}} & \frac{\partial \theta}{\partial \phi_{2}}
\end{array}\right) & =\left(\begin{array}{cc}
\hat{\alpha}\left(\phi_{1}, \phi_{2}\right) \phi_{1}+\hat{\beta}\left(\phi_{1}, \phi_{2}\right) \phi_{2} & \hat{\beta}\left(\phi_{1}, \phi_{2}\right) \phi_{1}+\hat{\gamma}\left(\phi_{1}, \phi_{2}\right) \phi_{2} \\
\frac{-\phi_{1}}{\phi_{1}^{2}+\phi_{2}^{2}} & \begin{array}{c}
\frac{\phi_{1}}{\phi_{1}^{2}+\phi_{2}^{2}} \\
\phi_{1}
\end{array}
\end{array}\right) \\
& =\frac{\hat{\alpha}\left(\phi_{1}, \phi_{2}\right) \phi_{1}^{2}+2 \hat{\beta}\left(\phi_{1}, \phi_{2}\right) \phi_{1} \phi_{2}+\hat{\gamma}\left(\phi_{1}, \phi_{2}\right) \phi_{2}^{2}}{\phi_{1}^{2}+\phi_{2}^{2}} \\
& =1+\frac{\left[\hat{\alpha}\left(\phi_{1}, \phi_{2}\right)-1\right] \phi_{1}^{2}+2 \hat{\beta}\left(\phi_{1}, \phi_{2}\right) \phi_{1} \phi_{2}+\left[\hat{\gamma}\left(\phi_{1}, \phi_{2}\right)-1\right] \phi_{2}^{2}}{\phi_{1}^{2}+\phi_{2}^{2}} \\
& =1+o(1)
\end{aligned}
$$

For the transition from $R, \theta$ to $x, y$

$$
\left(\begin{array}{ll}
\frac{\partial x}{\partial R} & \frac{\partial y}{\partial R} \\
\frac{\partial x}{\partial \theta} & \frac{\partial y}{\partial \theta}
\end{array}\right)=\left(\begin{array}{cc}
\frac{1}{\sqrt{2 R}} \cos \theta & \frac{1}{\sqrt{2 R}} \sin \theta \\
-\sqrt{2 R} \sin \theta & \sqrt{2 R} \cos \theta
\end{array}\right)=1
$$

\section{Appendix B. Sharp Volume Bounds}

In this Appendix we prove Theorem 1.1 under the assumptions $(\mathrm{H} 2)_{2,0}$ and (H3) (obviously, $(\mathrm{H} 2)_{k, h}$ implies $(\mathrm{H} 2)_{2,0}$ for all $k \geq 2$ and $h \geq 0$ ). The assumption (H5) will not be used in this proof. It will become clear 
in the proof that this estimate is best possible. Before going into the details we outline the strategy. Away from the critical points (in $\left.\theta_{1}, \theta_{2}\right)$ of the map $\eta=e\left(v_{1} \mathbf{p}\left(0, \theta_{1}\right)+v_{2} \mathbf{p}\left(0, \theta_{2}\right)+\mathbf{q}\right.$ ), the estimate is easily shown by a change of variables from one of the $\theta_{i}$ to $e$. Near the critical points, a detailed analysis of the singularity is required for getting the optimal improvement factor. We first determine for which $\mathbf{q}$ critical points are possible at all. Then we show that at any critical point the second derivative is a nonsingular matrix, thus the function $\eta$ is either of type $x^{2}+y^{2}$ or of type $x y$ (the factor $\log \varepsilon$ comes from the second case). We then use the Morse Lemma proven in Appendix A to calculate the volume improvement effect.

Note that $\mathbf{q} \in \mathcal{B}$ is not restricted to be near to $S$, so that we can make use of strict convexity only in the sense that on a fixed level set of $e$ near to $S$, there are only two solutions of the equation $\partial_{\theta} \mathbf{p}=\mathbf{v}$ for any $0 \neq \mathbf{v} \in \mathbb{R}^{d}$, and that the curvature radius is finite at every point of $S$. A convenient property of boundaries $S$ of strictly convex sets in $\mathbb{R}^{d}$, namely that no three different points of $S$ can lie on the same straight line, does not hold on the torus $\mathcal{B}$. The reason we could use it in Chapter 3 was that all momenta involved were on or near to $S$, and that we assumed (H5). The supremum in the definition of $\mathcal{W}$ is over all $\mathbf{q} \in \mathcal{B}$, not only those near to $S$, therefore (H5) would be of no use here. Most of the following Lemmas deal with the complications due to $\mathcal{B}$ being a torus.

\section{B.1 Two Dimensions}

Let $d=2$, use the coordinates $\rho$ and $\theta$ defined in Section 2.2, and let

$$
n(\rho, \theta)=\frac{\nabla e(\mathbf{p}(\rho, \theta))}{|\nabla e(\mathbf{p}(\rho, \theta))|}
$$

For brevity, we write $n(\theta)=n(0, \theta)$. The set

$$
E=\left\{\left(\theta_{1}, \theta_{2}\right) \in S \times S: n\left(\theta_{1}\right) \times n\left(\theta_{2}\right)=0\right\}
$$

is the zero set of the map

$$
H: S \times S \rightarrow \mathbb{R}, \quad\left(\theta_{1}, \theta_{2}\right) \mapsto\left(n\left(\theta_{1}\right) \times n\left(\theta_{2}\right)\right) \cdot e_{3}
$$

(where the vector product is inherited from $\mathbb{R}^{3}$ and $e_{3}$ denotes the unit vector in 3-direction). $H \in C^{1}(S \times$ $S, \mathbb{R})$, and

$$
\nabla H=\left(\frac{\partial H}{\partial \theta_{1}}, \frac{\partial H}{\partial \theta_{2}}\right)=\left(e_{3} \cdot\left(\partial_{\theta} n\left(\theta_{1}\right) \times n\left(\theta_{2}\right)\right), e_{3} \cdot\left(n\left(\theta_{1}\right) \times \partial_{\theta} n\left(\theta_{2}\right)\right)\right)
$$

is nonzero for all $\underline{\theta}=\left(\theta_{1}, \theta_{2}\right) \in E$ (because $n$ is a unit vector, $\partial_{\theta} n \perp n$, and because the Fermi surface has nonzero curvature, $\partial_{\theta} n \neq 0$ ). Thus $E$ is a $C^{1}$-submanifold of $S \times S$, of codimension 1 . Moreover, $E$ is compact, thus covered by finitely many balls in $S \times S$. Thus there is $\delta>0$ such that $U_{\delta}(E)$ is contained in this finite covering. Since $\mathcal{R}_{\delta}=S \times S \backslash U_{\delta}(E)$ is also compact, $|H(\underline{\theta})| \geq \varphi(\delta)>0$ holds for all $\underline{\theta} \notin U_{\delta}(E)$, with $\varphi$ some positive function $(\varphi(x)>0$ for $x>0)$. We shall choose $\delta>0$ later (independent of $\varepsilon$; this is the $\varepsilon$ of Theorem 1.1). Split

$$
\mathcal{W}(\varepsilon)=E_{\delta}(\varepsilon)+R_{\delta}(\varepsilon)
$$


according to the decomposition $S \times S=U_{\delta}(E) \dot{\cup} \mathcal{R}_{\delta}$. It was shown in Appendix A of I that the contribution $R_{\delta}$ from the regular region is bounded by

$$
R_{\delta}(\varepsilon) \leq \operatorname{const} \frac{\varepsilon}{\varphi(\delta)}
$$

The $1 / \varphi(\delta)$ comes from a Jacobian. To bound $E_{\delta}$, the contribution from the region where this Jacobian can become singular, we collect some more consequences of the geometry of the Fermi surface.

Lemma B.1 Let $\mathbf{p}=\mathbf{p}(\rho, \theta) \in \mathcal{U}(S)$. Then all solutions $\mathbf{Q}$ of

$$
\nabla e(\mathbf{Q}) \cdot \partial_{\theta} \mathbf{p}=0
$$

that satisfy $\mathbf{Q} \in \mathcal{U}(S)$ are given as $\mathbf{Q}=\mathbf{p}\left(\rho_{1}, \vartheta^{(k)}\left(\rho, \theta, \rho_{1}\right)\right)$ where for $k \in\{1,2\}, \vartheta^{(k)}$ are $C^{1}$ in $\left(\rho, \theta, \rho_{1}\right)$ and at $\rho_{1}=\rho$,

$$
\begin{aligned}
& \vartheta^{(1)}(\rho, \theta, \rho)=\theta \\
& \vartheta^{(2)}(\rho, \theta, \rho)=a_{\rho}(\theta) .
\end{aligned}
$$

At fixed $\rho$ and $\theta$, the curve $\rho_{1} \mapsto \vartheta^{(k)}\left(\rho, \theta, \rho_{1}\right)$ is transversal to $S_{\rho_{1}}$.

Proof: $\quad$ Since $\mathbf{Q} \in \mathcal{U}(S)$, we can write $\mathbf{Q}=\mathbf{p}\left(\rho_{1}, \theta_{1}\right)$. Fix $\rho$ and $\theta$. Since $S_{\rho_{1}}=\left\{\mathbf{p}\left(\rho_{1}, \theta_{1}\right): \theta_{1} \in[0,2 \pi]\right\}$ is strictly convex and a $C^{1}$-manifold, there are, for each fixed $\rho_{1}$, exactly two values of $\theta_{1}$ for which $\nabla e\left(\mathbf{p}\left(\rho_{1}, \theta_{1}\right)\right) \cdot \partial_{\theta} \mathbf{p}(\rho, \theta)=0$. For $\rho_{1}=\rho$ they are given by $\mathbf{p}(\rho, \theta)$ and $\mathbf{p}\left(\rho, a_{\rho}(\theta)\right)$. Fix $(\rho, \theta)$. The $\operatorname{map} \phi:\left(\rho_{1}, \theta_{1}\right) \mapsto \nabla e\left(\mathbf{p}\left(\rho_{1}, \theta_{1}\right)\right) \cdot \partial_{\theta} \mathbf{p}(\rho, \theta)$ satisfies $\phi(\rho, \theta)=0$ and $\phi\left(\rho, a_{\rho}(\theta)\right)=0$. The derivative

$$
\frac{\partial \phi}{\partial \theta_{1}}\left(\rho, \theta, \rho_{1}, \theta_{1}\right)=\left(\partial_{\theta} \mathbf{p}\left(\rho_{1}, \theta_{1}\right), e^{\prime \prime}\left(\mathbf{p}\left(\rho_{1}, \theta_{1}\right)\right) \partial_{\theta} \mathbf{p}(\rho, \theta)\right)
$$

is continuous. At $\theta_{1}=\theta$ and $\rho_{1}=\rho$, it is equal to $w(\mathbf{p}(\rho, \theta))$ (defined in (Sy)), hence nonzero. Thus in a neighbourhood of $\rho_{1}=\rho$ there is a function $\vartheta^{(1)}$ depending on $\left(\rho, \theta, \rho_{1}\right)$ such that $\phi\left(\rho_{1}, \vartheta^{(1)}\left(\rho, \theta, \rho_{1}\right)\right)=0$. Similarly, one constructs the solution $\vartheta^{(2)}$. $\phi$ is $C^{1}$ in $\rho_{1}, \rho, \theta$, so the $\vartheta^{(k)}$ are also $C^{1}$.

Transversality holds because $\theta_{1}$ moves $\mathbf{p}\left(\rho_{1}, \theta_{1}\right)$ tangentially to $S_{\rho_{1}}$ and $\frac{\partial \phi}{\partial \theta_{1}} \neq 0$.

Note that by compactness of $\mathcal{B}$ and $S$, the size of the neighbourhoods can be chosen uniform in $(\rho, \theta)$. We may therefore assume that $\mathcal{U}(S)$ is such that the solution curves of the Lemma B.1 exist for all $\mathbf{p} \in \mathcal{U}(S)$.

Lemma B.1 allows us to determine the set $\mathcal{P}_{\kappa}$ of those $\mathbf{q} \in \mathcal{B}$ for which critical points of the map

$$
\eta: \quad \underline{\theta}=\left(\theta_{1}, \theta_{2}\right) \mapsto \eta(\underline{\theta}, \mathbf{q})=e\left(v_{1} \mathbf{p}\left(0, \theta_{1}\right)+v_{2} \mathbf{p}\left(0, \theta_{2}\right)+\mathbf{q}\right)
$$

are possible. For $0<\kappa<r_{\mathrm{o}}$ let

$$
\mathcal{P}_{\kappa}=\bigcup_{k=1}^{2}\left\{\mathbf{p}\left(\rho, \vartheta^{(k)}\left(0, \theta_{1}, \rho\right)\right)-v_{1} \mathbf{p}\left(0, \theta_{1}\right)-v_{2} \mathbf{p}\left(0, \theta_{2}\right):\left(\theta_{1}, \theta_{2}\right) \in E,|\rho| \leq \kappa\right\}
$$




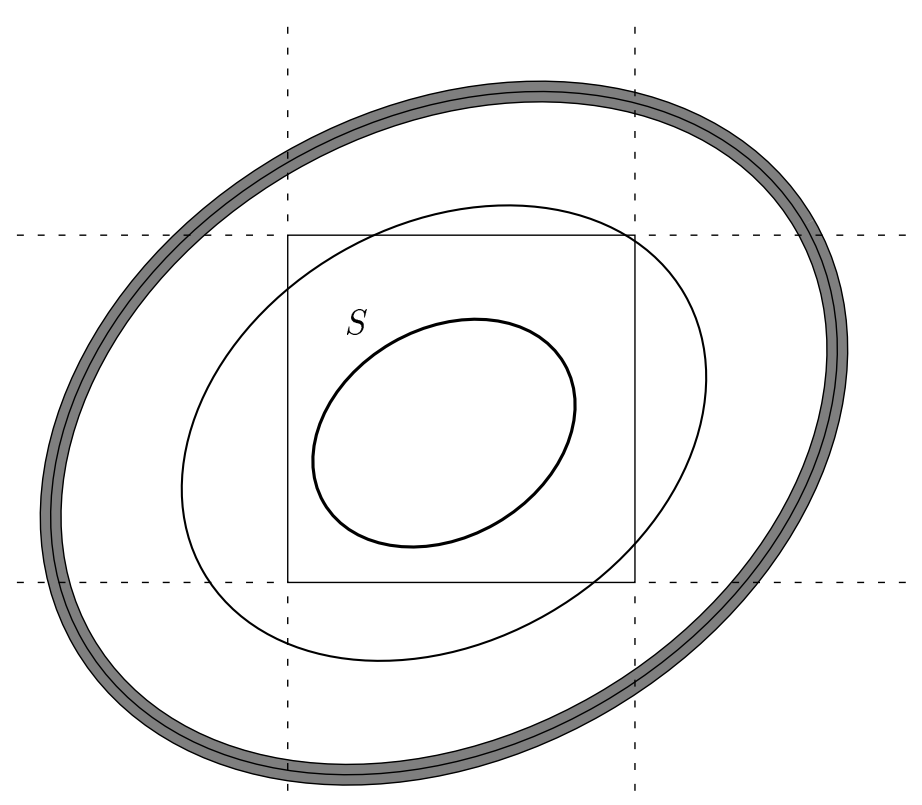

Figure 8

The set $\left\{\mathbf{p}\left(\rho, \vartheta^{(1)}(0, \theta, \rho)\right)+\mathbf{p}(0, \theta)+\mathbf{p}(0, \theta):|\rho| \leq \kappa\right\}$, which is one of the four sets in the union making up $\mathcal{P}_{\kappa}$, is sketched in Figure 8. The square indicates the boundary of the fundamental region for the torus $\mathcal{B}$, so the shaded region should be thought of as folded back into this fundamental region by periodicity.

Lemma B.2 If $\mathbf{q} \notin \mathcal{P}_{\kappa}$, then $|\eta(\underline{\theta}, \mathbf{q})| \leq \kappa$ implies $\frac{\partial \eta}{\partial \theta_{1}} \neq 0$ or $\frac{\partial \eta}{\partial \theta_{2}} \neq 0$.

Proof: Let

$$
\mathbf{Q}=v_{1} \mathbf{p}\left(0, \theta_{1}\right)+v_{2} \mathbf{p}\left(0, \theta_{2}\right)+\mathbf{q}
$$

The condition

$$
\frac{\partial \eta}{\partial \theta_{i}}=\nabla e(\mathbf{Q}) \cdot \partial_{\theta} \mathbf{p}\left(0, \theta_{i}\right)=0 \quad \text { for } i=1 \text { and } i=2
$$

implies $\underline{\theta} \in E$ : if $|e(\mathbf{Q})| \leq \kappa, \nabla e(\mathbf{Q}) \neq 0$, so $\partial_{\theta} \mathbf{p}\left(0, \theta_{1}\right)$ and $\partial_{\theta} \mathbf{p}\left(0, \theta_{2}\right)$ are both orthogonal to the same nonzero vector. Since $d=2$, they must be collinear, thus $\theta_{2} \in\left\{\theta_{1}, a\left(\theta_{1}\right)\right\}$ by strict convexity.

If there is a critical point, i.e. a solution to $(B .13)$ with $|e(\mathbf{Q})| \leq \kappa, \mathbf{Q}$ is determined as in Lemma B.1, so $\mathbf{q} \in \mathcal{P}_{\kappa}$ by $(B .12)$.

Fix $\kappa>0$. Without loss, we may assume that $\varepsilon \leq \frac{\kappa}{2}$ since the estimate in Theorem 1.1 is trivially true for $\varepsilon$ bounded away from zero by choice of the constant.

Since $\mathcal{P}_{\varepsilon} \subset \mathcal{P}_{\kappa / 2} \subset \mathcal{P}_{\kappa}$ and by continuity of $\frac{\partial \eta}{\partial \theta_{i}}$ and compactness, there is $\delta_{1}>0$ such that for all $\underline{\theta}$ in the support of the integral (1.4) for $\mathcal{W}$,

$$
\inf _{\mathbf{q} \in \overline{\mathcal{B} \backslash \mathcal{P}_{\kappa}}} \inf _{\theta \in E} \max \left\{\left|\frac{\partial \eta}{\partial \theta_{1}}\right|,\left|\frac{\partial \eta}{\partial \theta_{2}}\right|\right\} \geq \delta_{1} .
$$


Thus the $\delta>0$ introduced previously can be chosen so small (independently of $\varepsilon$ ) that for all $\mathbf{q} \notin \mathcal{P}_{\kappa}$ and all $\underline{\theta} \in U_{\delta}(E)$

$$
\left|\frac{\partial \eta}{\partial \theta_{i}}\right| \geq \delta_{1} \quad \text { for } i=1 \text { or } i=2 .
$$

Thus there are $R_{1}, R_{2}$ such that $U_{\delta}(E)=R_{1} \dot{\cup} R_{2}$ and $\left|\frac{\partial \eta}{\partial \theta_{i}}\right| \geq \delta_{1}$ on $R_{i}$. Obviously, $R_{1}$ and $R_{2}$ are similar, so we deal with $R_{1}$. By a change of variables from $\theta_{1}$ to $\eta$,

$$
\begin{aligned}
\sup _{\mathbf{q} \in \overline{\mathcal{B} \backslash \mathcal{P}_{\kappa}}} \int_{R_{1}} d \theta_{1} d \theta_{2} \mathbb{1}\left(\left|\eta\left(\theta_{1}, \theta_{2}, \mathbf{q}\right)\right| \leq \varepsilon\right) & \leq 2 \pi \frac{1}{\delta_{1}} \int d \eta \mathbb{1}(|\eta| \leq \varepsilon) \leq \\
& \leq 4 \pi \frac{\varepsilon}{\delta_{1}},
\end{aligned}
$$

the factor of $2 \pi$ coming from the $\theta_{2}$ integration. Thus, to prove Theorem 1.1 it suffices to bound

$$
W_{\kappa}(\varepsilon)=\max _{v_{i} \in\{ \pm 1\}} \sup _{\mathbf{q} \in \mathcal{P}_{\kappa}\left(v_{1}, v_{2}\right)} \int_{U_{\delta}(E)} d \theta_{1} d \theta_{2} \mathbb{1}(|\eta(\underline{\theta}, \mathbf{q})| \leq \varepsilon) .
$$

Lemma B.3 For fixed $\mathbf{q} \in \mathcal{P}_{\kappa}$, the solutions $\underline{\theta}=\underline{\theta}^{(c r)}$ of (B.13) are isolated. The critical points $\underline{\theta}^{c r}$ are $C^{1}$ functions of $\mathbf{q}$.

Proof: Case $\mathbf{p}_{2}=\mathbf{p}_{1}$ : the right side of the equation

$$
\mathbf{q}=\mathbf{p}\left(\rho, \vartheta^{(k)}(0, \theta, \rho)\right)-v_{1} \mathbf{p}(0, \theta)-v_{2} \mathbf{p}(0, \theta)
$$

is a $C^{1}$ function of $(\rho, \theta)$. We now show that this function is invertible. The derivatives are

$$
\begin{aligned}
& \frac{\partial \mathbf{q}}{\partial \rho}=\partial_{\rho} \mathbf{p}\left(\rho, \vartheta^{(k)}\left(0, \theta_{1}, \rho\right)\right)+\partial_{\theta} \mathbf{p}\left(\rho, \vartheta^{(k)}\left(0, \theta_{1}, \rho\right)\right) \frac{\partial \vartheta^{(k)}}{\partial \rho} \\
& \frac{\partial \mathbf{q}}{\partial \theta}=\partial_{\theta} \mathbf{p}\left(\rho, \vartheta^{(k)}\left(0, \theta_{1}, \rho\right)\right) \frac{\partial \vartheta^{(k)}}{\partial \theta}-v_{1} \partial_{\theta} \mathbf{p}(0, \theta)-v_{2} \partial_{\theta} \mathbf{p}(0, \theta)
\end{aligned}
$$

We know that $\rho$ is in a small neighbourhood of zero by Lemma B.1, so by continuity in $\rho$, it suffices to prove that the derivative is nonsingular at $\rho=0$.

Case $\vartheta^{(1)}$ : At $\rho=0, \vartheta^{(1)}=\theta$, so

$$
\begin{aligned}
& \frac{\partial \mathbf{q}}{\partial \rho}=\partial_{\rho} \mathbf{p}(\theta)+\partial_{\theta} \mathbf{p}(\theta) \frac{\partial \vartheta^{(1)}}{\partial \rho} \\
& \frac{\partial \mathbf{q}}{\partial \theta}=\partial_{\theta} \mathbf{p}(\theta)\left(\frac{\partial \vartheta^{(1)}}{\partial \theta}-v_{1}-v_{2}\right)
\end{aligned}
$$

From (B.8), we see that

$$
\frac{\partial \vartheta^{(1)}}{\partial \theta}(\rho, \theta, \rho)=1
$$


so $\frac{\partial \vartheta^{(1)}}{\partial \theta}(\rho, \theta, \rho)-v_{1}-v_{2}$ is $-1,1$ or $3 . \frac{\partial \vartheta^{(1)}}{\partial \theta}$ is continuous, so there is $r_{1}$ such that for $\left|\rho_{1}-\rho\right|<r_{1}$,

$$
\left|\frac{\partial \vartheta^{(1)}}{\partial \theta}\left(\rho, \theta, \rho_{1}\right)-v_{1}-v_{2}\right| \geq \frac{1}{2}
$$

It is now obvious that $\operatorname{rank}\left(\frac{\partial \mathbf{q}}{\partial \rho}, \frac{\partial \mathbf{q}}{\partial \theta}\right)=2$ : the first column is nonzero because $\partial_{\rho} \mathbf{p}(0, \theta)$ is nonzero, and $\partial_{\rho} \mathbf{p}(0, \theta)$ and $\partial_{\theta} \mathbf{p}(0, \theta)$ are linearly independent. The second column is a nonzero multiple of $\partial_{\theta} \mathbf{p}(0, \theta)$. Thus the column vectors are linearly independent.

Case $\vartheta^{(2)}$ : Recall that $\vartheta^{(2)}$ is the solution with $\vartheta^{(2)}(\rho, \theta, \rho)=a_{\rho}(\theta)$. Hence

$$
\frac{\partial \vartheta^{(2)}}{\partial \theta}(\rho, \theta, \rho)=\frac{\partial a_{\rho}(\theta)}{\partial \theta}
$$

Thus, by (B.19), at $\rho=0$

$$
\begin{aligned}
\frac{\partial \mathbf{q}}{\partial \rho} & =\partial_{\rho} \mathbf{p}(a(\theta))+\partial_{\theta} \mathbf{p}(\theta) \frac{\partial \vartheta^{(2)}}{\partial \rho}(0, \theta, \rho=0) \\
\frac{\partial \mathbf{q}}{\partial \theta} & =\partial_{\theta} \mathbf{p}(a(\theta)) \frac{\partial \vartheta^{(2)}}{\partial \theta}(0, \theta, 0)-v_{1} \partial_{\theta} \mathbf{p}(\theta)-v_{2} \partial_{\theta} \mathbf{p}(\theta) \\
& =\partial_{\theta} \mathbf{p}(\theta)\left(-\frac{\partial a}{\partial \theta}(\theta)-v_{1}-v_{2}\right)
\end{aligned}
$$

By (2.35), $\frac{\partial \mathbf{q}}{\partial \theta} \neq 0$. So, similarly to the first case, the two rows are linearly independent.

Case $\theta_{2}=a\left(\theta_{1}\right)$ : The argument is similar to the above, only that now in the case $\vartheta^{(1)}$,

$$
\frac{\partial \mathbf{q}}{\partial \theta}(0, \theta)=\partial_{\theta} \mathbf{p}(0, \theta)\left(\frac{\partial \vartheta^{(1)}}{\partial \theta}(0, \theta, 0)-v_{1}+v_{2} \frac{\partial a}{\partial \theta}(\theta)\right)
$$

and in the case $\vartheta^{(2)}$,

$$
\frac{\partial \mathbf{q}}{\partial \theta}(0, \theta)=\partial_{\theta} \mathbf{p}(0, \theta)\left(-\frac{\partial \vartheta^{(2)}}{\partial \theta}(0, \theta, 0)-v_{1}+v_{2} \frac{\partial a}{\partial \theta}(\theta)\right)
$$

Remark B.4 By compactness of $\overline{\mathcal{P}_{\kappa}}$, the critical points are uniformly continuous functions of $\mathbf{q}$. Thus, given $s>0$, there is $r>0$ such that for all $\mathbf{q} \in \mathcal{P}_{\kappa}$ and all $\mathbf{q}^{\prime} \in U_{r}(\mathbf{q}),\left|\underline{\theta}^{c r}\left(\mathbf{q}^{\prime}\right)-\underline{\theta}^{c r}(\mathbf{q})\right|<s / 2$. Moreover, there is a finite set of points $\mathbf{q}^{(1)}, \ldots, \mathbf{q}^{(n)}$ such that $\mathcal{P}_{\kappa} \subset \bigcup_{i=1}^{n} U_{r}\left(\mathbf{q}^{(i)}\right)$.

Proof of Theorem 1.1 for $d=2$ : It suffices to prove (B.17) for any given $i . s$ will be chosen at the end. $n$ depends on $s$. Let $i \in\{1, \ldots, n\}, \mathbf{q} \in U_{r}\left(\mathbf{q}^{(i)}\right)$, and let

$$
K_{i}=E \backslash \bigcup_{l=1}^{4} U_{s}\left(\underline{\theta}^{c r}\left(\mathbf{q}^{(i)}\right)\right) .
$$


If $\underline{\theta} \notin K_{i}, \frac{\partial \eta}{\partial \theta_{1}}(\underline{\theta}, \mathbf{q}) \neq 0$ or $\frac{\partial \eta}{\partial \theta_{2}}(\underline{\theta}, \mathbf{q}) \neq 0$ because by choice of $r$, all critical points to $\mathbf{q} \in U_{r}\left(\mathbf{q}^{(i)}\right)$ are in $U_{s / 2}\left(\underline{\theta}^{c r}\left(\mathbf{q}^{(i)}\right)\right)$. Thus, by a similar argument as in the case $\mathbf{q} \notin \mathcal{P}_{\kappa}$,

$$
\sup _{\mathbf{q} \in U_{r}\left(\mathbf{q}^{(i)}\right)} \int_{K_{i}} d \theta_{1} d \theta_{2} \mathbb{1}\left(\left|\eta\left(\theta_{1}, \theta_{2}, \mathbf{q}\right)\right| \leq \varepsilon\right) \leq \operatorname{const} \frac{\varepsilon}{\delta_{2}}
$$

where $\delta_{2}$ does not depend on $\varepsilon$.

Finally, let $\underline{t}=\underline{t}(\mathbf{q})$ be one of the critical points to $\mathbf{q}$ and $\underline{\theta} \in U_{s}(\underline{t})$. We now have to bound

$$
W_{\kappa}^{(i)}(\varepsilon)=\max _{v_{1}, v_{2}= \pm 1} \sup _{\mathbf{q} \in U_{r}\left(\mathbf{q}^{(i)}\right) \cap \mathcal{P}_{\kappa}} \int_{U_{s}(\underline{t}(\mathbf{q}))} d \theta_{1} d \theta_{2} \mathbb{1}(|\eta(\underline{\theta}, \mathbf{q})| \leq \varepsilon) .
$$

By Taylor expansion,

$$
\eta(\underline{\theta}, \mathbf{q})=\eta(\underline{t}, \mathbf{q})+(\underline{\theta}-\underline{t}, D(\underline{t}, \underline{\theta}-\underline{t}, \mathbf{q})(\underline{\theta}-\underline{t}))
$$

with

$$
D(\underline{t}, \underline{\phi}, \mathbf{q})=\int_{0}^{1} d \alpha(1-\alpha) \quad\left(\begin{array}{cc}
\partial_{1}{ }^{2} \eta(\underline{t}+\alpha \underline{\phi}, \mathbf{q}) & \partial_{1} \partial_{2} \eta(\underline{t}+\alpha \underline{\phi}, \mathbf{q}) \\
\partial_{2} \partial_{1} \eta(\underline{t}+\alpha \underline{\phi}, \mathbf{q}) & \partial_{2}{ }^{2} \eta(\underline{t}+\alpha \underline{\phi}, \mathbf{q})
\end{array}\right) .
$$

Let

$$
D_{\mathrm{o}}=D_{\mathrm{o}}(\mathbf{q})=D(\underline{t}(\mathbf{q}), 0,0, \mathbf{q})
$$

Lemma B.5 For all $\mathbf{q} \in U_{r}\left(\mathbf{q}^{(i)}\right)$, $\left|\operatorname{det} D_{\mathrm{o}}(\mathbf{q})\right| \geq \frac{1}{4} w_{\mathrm{o}}^{2}$.

Proof: $\quad$ By (B.12) and (B.10),

$$
\begin{aligned}
\partial_{1}^{2} \eta(\underline{\theta}, \mathbf{q}) & =\left(\partial_{\theta} \mathbf{p}_{1}, e^{\prime \prime}(\mathbf{Q}) \partial_{\theta} \mathbf{p}_{1}\right)+v_{1} \nabla e(\mathbf{Q}) \cdot \partial_{\theta}^{2} \mathbf{p}_{1} \\
\partial_{2}^{2} \eta(\underline{\theta}, \mathbf{q}) & =\left(\partial_{\theta} \mathbf{p}_{2}, e^{\prime \prime}(\mathbf{Q}) \partial_{\theta} \mathbf{p}_{2}\right)+v_{2} \nabla e(\mathbf{Q}) \cdot \partial_{\theta}^{2} \mathbf{p}_{2} \\
\partial_{1} \partial_{2} \eta(\underline{\theta}, \mathbf{q}) & =v_{1} v_{2}\left(\partial_{\theta} \mathbf{p}_{2}, e^{\prime \prime}(\mathbf{Q}) \partial_{\theta} \mathbf{p}_{1}\right)=\partial_{2} \partial_{1} \eta(\underline{\theta}, \mathbf{q}) .
\end{aligned}
$$

$\eta$ is $C^{2}$, so $D$ is continuous in all its arguments. We set $\underline{\theta}$ to its critical value $\underline{t}$. Then $\mathbf{Q}=\mathbf{p}\left(\rho_{3}, \vartheta^{(k)}\left(0, \theta_{1}, \rho_{3}\right)\right)$. By continuity of $D$, it suffices to show that $\left|\operatorname{det} D_{\mathrm{o}}\right| \geq \frac{3}{8} w_{\mathrm{o}}^{2}$ for $\rho_{3}=0$. Thus we can put $\rho_{3}=0$. Then $\vartheta^{(1)}=\theta_{1}$ and $\vartheta^{(2)}=a\left(\theta_{1}\right)$. Let $\mathbf{q} \in U_{r}\left(\mathbf{q}^{(i)}\right) \cap \mathcal{P}_{\kappa}$, and let $\underline{t}$ be the critical point for $\mathbf{q}$. Recall that $w(\mathbf{p})=\left(\partial_{\theta} \mathbf{p}, e^{\prime \prime}(\mathbf{p}) \partial_{\theta} \mathbf{p}\right)$, and that $\mathbf{q}=\mathbf{Q}-v_{1} \mathbf{p}_{1}-v_{2} \mathbf{p}_{2}$.

Case $\vartheta^{(1)}: \mathbf{Q}=\mathbf{p}\left(0, \theta_{1}\right)=\mathbf{p}_{1}$. If $\theta_{2}=\theta_{1}$, i.e. $\mathbf{p}_{2}=\mathbf{p}_{1}=\mathbf{p}$,

$$
D_{\mathrm{o}}=\left(\begin{array}{cc}
w(\mathbf{p})\left(1-v_{1}\right) & v_{1} v_{2} w(\mathbf{p}) \\
v_{1} v_{2} w(\mathbf{p}) & w(\mathbf{p})\left(1-v_{2}\right)
\end{array}\right)
$$

If $v_{1}=v_{2}=-1, \operatorname{det} D_{\mathrm{o}}=3 w(\mathbf{p})^{2}>0$, and $\mathbf{q}=3 \mathbf{p}$. Otherwise, $\operatorname{det} D_{\mathrm{o}}=-w(\mathbf{p})^{2}<0$.

If $\theta_{2}=a\left(\theta_{1}\right)$, we use (2.36) and (2.24), to get

$$
\nabla e(\mathbf{p}(0, \theta)) \cdot\left(\partial_{\theta}^{2} \mathbf{p}\right)(0, a(\theta))=-\frac{1}{\frac{\partial a}{\partial \theta}(\theta)} \nabla e(\mathbf{p}(0, \theta)) \cdot\left(\partial_{\theta}^{2} \mathbf{p}\right)(0, \theta)=\frac{1}{\frac{\partial a}{\partial \theta}(\theta)} w(\mathbf{p}(0, \theta)) .
$$


Thus we can rewrite $D_{\mathrm{o}}$ as

$$
D_{\mathrm{o}}=w\left(\mathbf{p}_{1}\right)\left(\begin{array}{cc}
\left(1-v_{1}\right) & -v_{1} v_{2} \\
-v_{1} v_{2} & \left(1+v_{2} \frac{1}{\frac{\partial a}{\partial \theta}\left(\theta_{1}\right)}\right)
\end{array}\right)
$$

If $v_{1}=1, \operatorname{det} D_{\mathrm{o}}=-w\left(\mathbf{p}_{1}\right)^{2}$. If $v_{1}=-1$,

$$
\operatorname{det} D_{\mathrm{o}}=w\left(\mathbf{p}_{1}\right)^{2}\left(2\left(1+\frac{v_{2}}{\frac{\partial a}{\partial \theta}}\right)-1\right)
$$

so if $v_{2}=1, \operatorname{det} D_{\mathrm{o}} \geq \frac{25}{9} w\left(\mathbf{p}_{1}\right)^{2}$. If $v_{2}=-1$,

$$
\left(1+\frac{v_{2}}{\frac{\partial a}{\partial \theta}}\right)=\left(1-\frac{1}{\frac{\partial a}{\partial \theta}}\right) \leq \frac{1}{9}
$$

by $(2.35)$, so $\operatorname{det} D_{\mathrm{o}} \leq-\frac{3}{4} w\left(\mathbf{p}_{\mathbf{1}}\right)^{2}$.

Case $\vartheta^{(2)}: \mathbf{Q}=\mathbf{p}\left(0, a\left(\theta_{1}\right)\right)=\mathbf{a}\left(\mathbf{p}_{1}\right)$. If $\mathbf{p}_{2}=\mathbf{p}_{1}$,

$$
D_{\mathrm{o}}=\left(\begin{array}{cc}
\left(\partial_{\theta} \mathbf{p}_{\mathbf{1}}, e^{\prime \prime}\left(a\left(\mathbf{p}_{\mathbf{1}}\right)\right) \partial_{\theta} \mathbf{p}_{\mathbf{1}}\right)+v_{1} \nabla e\left(a\left(\mathbf{p}_{1}\right)\right) \cdot \partial_{\theta}^{2} \mathbf{p}_{\mathbf{1}} & v_{1} v_{2}\left(\partial_{\theta} \mathbf{p}_{2}, e^{\prime \prime}\left(a\left(\mathbf{p}_{1}\right)\right) \partial_{\theta} \mathbf{p}_{\mathbf{1}}\right) \\
\left(\partial_{\theta} \mathbf{p}_{\mathbf{1}}, e^{\prime \prime}\left(a\left(\mathbf{p}_{\mathbf{1}}\right)\right) \partial_{\theta} \mathbf{p}_{\mathbf{2}}\right) & \left(\partial_{\theta} \mathbf{p}_{\mathbf{1}}, e^{\prime \prime}\left(a\left(\mathbf{p}_{\mathbf{1}}\right)\right) \partial_{\theta} \mathbf{p}_{1}\right)+v_{1} \nabla e\left(a\left(\mathbf{p}_{\mathbf{1}}\right)\right) \cdot \partial_{\theta}^{2} \mathbf{p}_{\mathbf{1}}
\end{array}\right)
$$

We use (2.34) and

to rewrite this as

$$
\begin{aligned}
\nabla e(\mathbf{p}(a(\theta))) \cdot\left(\partial_{\theta}^{2} \mathbf{p}\right)(\theta) & =-\frac{\partial a}{\partial \theta} \nabla e(\mathbf{p}(a(\theta))) \cdot\left(\partial_{\theta}^{2} \mathbf{p}\right)(a(\theta))= \\
& =\frac{\partial a}{\partial \theta} w(\mathbf{p}(a(\theta)))
\end{aligned}
$$

$$
D_{\mathrm{o}}=w\left(a\left(\mathbf{p}_{1}\right)\right)\left(\begin{array}{cc}
1+v_{1} \frac{\partial a}{\partial \theta}\left(\theta_{1}\right) & v_{1} v_{2} \\
v_{1} v_{2} & 1+v_{2} \frac{\partial a}{\partial \theta}\left(\theta_{1}\right)
\end{array}\right)
$$

For all $v_{1}= \pm 1$ and $v_{2}= \pm 1,\left|\operatorname{det} D_{\mathrm{o}}\right| \geq \frac{3}{8} w_{\mathrm{o}}^{2}$ by $(2.35)$.

$$
\text { If } \mathbf{p}_{2}=\mathbf{a}\left(\mathbf{p}_{1}\right)
$$

$$
D_{\mathrm{o}}=w\left(a\left(\mathbf{p}_{1}\right)\right)\left(\begin{array}{cc}
\left(1+v_{1} \frac{\partial a}{\partial \theta}\left(\theta_{1}\right)\right) & -v_{1} v_{2} \\
-v_{1} v_{2} & 1-v_{2}
\end{array}\right)
$$

For all $v_{1}= \pm 1$ and $v_{2}= \pm 1,\left|\operatorname{det} D_{\mathrm{o}}\right| \geq \frac{3}{4} w_{\mathrm{o}}^{2}$ by $(2.35)$.

Fix $\mathbf{q} \in U_{r}\left(\mathbf{q}^{(i)}\right)$. Let $\mathcal{O}(\mathbf{q})$ be the rotation such that $\mathcal{O}(\mathbf{q}) D_{\mathrm{o}}(\mathbf{q}) \mathcal{O}(\mathbf{q})^{-1}=\tilde{D}_{\mathrm{o}}=\operatorname{diag}\left\{d_{1}, d_{2}\right\}$. Rotate $\underline{\theta}-\underline{t} \rightarrow \underline{\tilde{\phi}}=\mathcal{O}(\mathbf{q})(\underline{\theta}-\underline{t})$. This change of variables is $C^{\infty}$ and the Jacobian is one. Denoting $\tilde{D}(\underline{t}, \underline{\hat{\phi}}, \mathbf{q})=$ $\mathcal{O}(\mathbf{q}) D\left(\underline{t}, \mathcal{O}(\mathbf{q})^{-1} \tilde{\phi}, \mathbf{q}\right) \mathcal{O}(\mathbf{q})^{-1}$, we are going to bound

$$
\int_{U_{s}(0,0)} d \tilde{\phi}_{1} d \tilde{\phi}_{2} \mathbb{1}(|\eta(\mathbf{t}(\mathbf{q}), \mathbf{q})+(\underline{\tilde{\phi}}, \tilde{D}(\underline{t}, \underline{\tilde{\phi}}, \mathbf{q}) \underline{\tilde{\phi}})| \leq \varepsilon)
$$

uniformly in $\mathbf{q}$. We rescale

$$
\left(\tilde{\phi}_{1}, \tilde{\phi}_{2}\right) \rightarrow\left(\phi_{1}, \phi_{2}\right)=\left(\left|d_{1}\right|^{\frac{1}{2}} \tilde{\phi}_{1},\left(\left|d_{2}\right|^{\frac{1}{2}} \tilde{\phi}_{2}\right)\right.
$$

This transformation is again $C^{\infty}$, and its Jacobian is

$$
\left|\operatorname{det} D_{\mathrm{o}}\right|^{-\frac{1}{2}} \leq \frac{2}{\left|w_{\mathrm{o}}\right|}
$$


Thus, in the new coordinates,

$$
\eta(\underline{\theta}, \mathbf{q})-\eta(\underline{t}(\mathbf{q}), \mathbf{q})=\nu\left(\phi_{1}, \phi_{2}\right)
$$

with a function $\nu \in C^{2}$, since $\eta \in C^{2}$ and the coordinate change from $\underline{\theta}$ to $\underline{\phi}$ is $C^{\infty}$. Moreover,

$$
\begin{aligned}
\nu(0,0) & =0, \quad \partial_{i} \nu(0,0)=0, \quad \partial_{1} \partial_{2} \nu(0,0)=0, \\
\partial_{1}^{2} \nu(0,0) & = \pm 1, \quad \partial_{1}^{2} \nu(0,0)= \pm 1,
\end{aligned}
$$

and $\underline{\theta} \in U_{s}(\mathbf{t})$ implies $\underline{\phi} \in U_{\sigma}(0,0)$ for some $\sigma(s)>0$. Thus we have proven the statement if we can bound

$$
\int_{U_{\sigma}(0,0)} d \phi_{1} d \phi_{2} \mathbb{1}\left(\left|f_{\mathrm{o}}+\nu\left(\phi_{1}, \phi_{2}\right)\right| \leq \varepsilon\right)
$$

uniformly in $f_{\mathrm{o}}$ (here $f_{\mathrm{o}}=\eta(\underline{t}(\mathbf{q}), \mathbf{q})$ ). Because of the absolute value in $(B .48)$, we may assume $\partial_{1}^{2} \nu=1$, $\partial_{2}^{2} \nu= \pm 1$. If $\partial_{2}^{2} \nu=1$, by Theorem A.3 there is a change of variables $\left(\phi_{1}, \phi_{2}\right) \rightarrow(R, \alpha)$ with Jacobian bounded by 2 if $s$ is chosen small enough, such that $\nu\left(\phi_{1}, \phi_{2}\right)=R$. Thus (B.48) is bounded by

$$
2 \int d R d \alpha \mathbb{1}\left(\left|f_{\mathrm{o}}+R\right| \leq \varepsilon\right) \leq 8 \pi \varepsilon
$$

If $\partial_{2}^{2} \nu=-1$, by Theorem A.2, there is a change of variables $\left(\phi_{1}, \phi_{2}\right) \rightarrow(x, y)$, such that $\nu\left(\phi_{1}, \phi_{2}\right)=x y$, and therefore (B.48) is bounded by

$$
\int_{U_{\tilde{\sigma}}(0,0)} d x d y \mathbb{1}\left(\left|f_{\mathrm{o}}+x y\right| \leq \varepsilon\right) \leq \mathrm{const}|\log \tilde{\sigma}| \varepsilon|\log \varepsilon|
$$

where $\tilde{\sigma}$ is such that the image of $U_{\sigma}(0,0)$ under the last change of variables is contained in $U_{\tilde{\sigma}}(0,0)$. This completes the proof of Theorem 1.1 in two dimensions.

\section{B.2 Higher Dimensions}

In this Section, we prove Theorem 1.1 for $d \geq 3$. The method of proof is a generalization of that for $d=2$. The main change is that instead of numbers, matrices of dimension $d-1$ appear.

Lemma B.6 (Nondegeneracy of Critical Points) Fix any $\mathbf{q} \in \mathbb{R}^{d}$ and $v_{\theta}, v_{\phi} \in\{ \pm 1\}$. Suppose that $\left(\theta_{0}, \phi_{0}\right) \in S^{d-1} \times S^{d-1}$ is a critical point of $F(\theta, \phi)=e\left(\mathbf{q}+v_{\theta} \mathbf{p}(0, \theta)+v_{\phi} \mathbf{p}(0, \phi)\right)$ and that $e\left(\mathbf{q}+v_{\theta} \mathbf{p}\left(0, \theta_{0}\right)+\right.$ $\left.v_{\phi} \mathbf{p}\left(0, \phi_{\mathrm{o}}\right)\right)=0$. Then $\partial^{2} F(\theta, \phi)$ has a nonzero determinant for $\theta=\theta_{\mathrm{o}}, \phi=\phi_{\mathrm{o}}$.

Proof: Abuse notation by replacing $\mathbf{p}(0, \theta)$ with $\mathbf{p}(\theta)$. Define $\alpha_{\mathrm{o}}$ by $\mathbf{p}\left(\alpha_{\mathrm{o}}\right)=\mathbf{q}+v_{\theta} \mathbf{p}\left(\theta_{\mathrm{o}}\right)+v_{\phi} \mathbf{p}\left(\phi_{0}\right)$. That $\left(\theta_{0}, \phi_{0}\right)$ is a critical point of $F$ means

$$
\nabla e\left(\mathbf{p}\left(\alpha_{\mathrm{o}}\right)\right) \cdot \partial_{i} \mathbf{p}\left(\theta_{\mathrm{o}}\right)=\nabla e\left(\mathbf{p}\left(\alpha_{\mathrm{o}}\right)\right) \cdot \partial_{j} \mathbf{p}\left(\phi_{\mathrm{o}}\right)=0
$$


for all $i, j \in\{1, \ldots, d-1\}$. Hence the normal vectors to the Fermi surface at $\mathbf{p}\left(\theta_{0}\right)$ and $\mathbf{p}\left(\phi_{0}\right)$ must be parallel to the normal vector to the Fermi surface at $\mathbf{p}\left(\alpha_{\mathrm{o}}\right)$. In other words, there are nonzero numbers $c_{\theta}, c_{\phi}$ such that

$$
\begin{aligned}
& \nabla e\left(\mathbf{p}\left(\alpha_{\mathrm{o}}\right)\right)=c_{\theta} \nabla e\left(\mathbf{p}\left(\theta_{\mathrm{o}}\right)\right) \\
& \nabla e\left(\mathbf{p}\left(\alpha_{\mathrm{o}}\right)\right)=c_{\phi} \nabla e\left(\mathbf{p}\left(\phi_{\mathrm{o}}\right)\right)
\end{aligned}
$$

For all $\alpha, i, j$

$$
\begin{aligned}
& e(\mathbf{p}(\alpha))=0 \\
& \nabla e(\mathbf{p}(\alpha)) \cdot \partial_{i} \mathbf{p}(\alpha)=0 \\
& \left(\partial_{j} \mathbf{p}(\alpha), \partial^{2} e(\mathbf{p}(\alpha)) \partial_{i} \mathbf{p}(\alpha)\right)+\nabla e(\mathbf{p}(\alpha)) \cdot \partial_{j} \partial_{i} \mathbf{p}(\alpha)=0
\end{aligned}
$$

Hence

$$
\begin{aligned}
\partial_{\theta_{i}} \partial_{\theta_{j}} F\left(\theta_{\mathrm{o}}, \phi_{\mathrm{o}}\right) & =\left(\partial_{j} \mathbf{p}\left(\theta_{\mathrm{o}}\right), \partial^{2} e\left(\mathbf{p}\left(\alpha_{\mathrm{o}}\right)\right) \partial_{i} \mathbf{p}\left(\theta_{\mathrm{o}}\right)\right)+v_{\theta} \nabla e\left(\mathbf{p}\left(\alpha_{\mathrm{o}}\right)\right) \cdot \partial_{j} \partial_{i} \mathbf{p}\left(\theta_{\mathrm{o}}\right) \\
& =\left(\partial_{j} \mathbf{p}\left(\theta_{\mathrm{o}}\right), \partial^{2} e\left(\mathbf{p}\left(\alpha_{\mathrm{o}}\right)\right) \partial_{i} \mathbf{p}\left(\theta_{\mathrm{o}}\right)\right)+v_{\theta} c_{\theta} \nabla e\left(\mathbf{p}\left(\theta_{\mathrm{o}}\right)\right) \cdot \partial_{j} \partial_{i} \mathbf{p}\left(\theta_{\mathrm{o}}\right) \\
& =\left(\partial_{j} \mathbf{p}\left(\theta_{\mathrm{o}}\right), \partial^{2} e\left(\mathbf{p}\left(\alpha_{\mathrm{o}}\right)\right) \partial_{i} \mathbf{p}\left(\theta_{\mathrm{o}}\right)\right)-v_{\theta} c_{\theta}\left(\partial_{j} \mathbf{p}\left(\theta_{\mathrm{o}}\right), \partial^{2} e\left(\mathbf{p}\left(\theta_{\mathrm{o}}\right)\right) \partial_{i} \mathbf{p}\left(\theta_{\mathrm{o}}\right)\right) \\
\partial_{\phi_{i}} \partial_{\phi_{j}} F\left(\phi_{\mathrm{o}}, \phi_{\mathrm{o}}\right) & =\left(\partial_{j} \mathbf{p}\left(\phi_{\mathrm{o}}\right), \partial^{2} e\left(\mathbf{p}\left(\alpha_{\mathrm{o}}\right)\right) \partial_{i} \mathbf{p}\left(\phi_{\mathrm{o}}\right)\right)+v_{\phi} \nabla e\left(\mathbf{p}\left(\alpha_{\mathrm{o}}\right)\right) \cdot \partial_{j} \partial_{i} \mathbf{p}\left(\phi_{\mathrm{o}}\right) \\
& =\left(\partial_{j} \mathbf{p}\left(\phi_{\mathrm{o}}\right), \partial^{2} e\left(\mathbf{p}\left(\alpha_{\mathrm{o}}\right)\right) \partial_{i} \mathbf{p}\left(\phi_{\mathrm{o}}\right)\right)+v_{\phi} c_{\phi} \nabla e\left(\mathbf{p}\left(\phi_{\mathrm{o}}\right)\right) \cdot \partial_{j} \partial_{i} \mathbf{p}\left(\phi_{\mathrm{o}}\right) \\
& =\left(\partial_{j} \mathbf{p}\left(\phi_{\mathrm{o}}\right), \partial^{2} e\left(\mathbf{p}\left(\alpha_{\mathrm{o}}\right)\right) \partial_{i} \mathbf{p}\left(\phi_{\mathrm{o}}\right)\right)-v_{\phi} c_{\phi}\left(\partial_{j} \mathbf{p}\left(\phi_{\mathrm{o}}\right), \partial^{2} e\left(\mathbf{p}\left(\phi_{\mathrm{o}}\right)\right) \partial_{i} \mathbf{p}\left(\phi_{\mathrm{o}}\right)\right) \\
\partial_{\theta_{i}} \partial_{\phi_{j}} F\left(\theta_{\mathrm{o}}, \phi_{\mathrm{o}}\right) & =v_{\theta} v_{\phi}\left(\partial_{j} \mathbf{p}\left(\phi_{\mathrm{o}}\right), \partial^{2} e\left(\mathbf{p}\left(\alpha_{\mathrm{o}}\right)\right) \partial_{i} \mathbf{p}\left(\theta_{\mathrm{o}}\right)\right)
\end{aligned}
$$

Define the $(d-1) \times(d-1)$ matrices

$$
\begin{aligned}
M_{\alpha} & =\left[\left(\partial_{j} \mathbf{p}\left(\theta_{\mathrm{o}}\right), \partial^{2} e\left(\mathbf{p}\left(\alpha_{\mathrm{o}}\right)\right) \partial_{i} \mathbf{p}\left(\theta_{\mathrm{o}}\right)\right)\right]_{1 \leq i, j \leq d-1} \\
M_{\theta} & =\left[\left(\partial_{j} \mathbf{p}\left(\theta_{\mathrm{o}}\right), \partial^{2} e\left(\mathbf{p}\left(\theta_{\mathrm{o}}\right)\right) \partial_{i} \mathbf{p}\left(\theta_{\mathrm{o}}\right)\right)\right]_{1 \leq i, j \leq d-1} \\
M_{\phi} & =\left[\left(\partial_{j} \mathbf{p}\left(\theta_{\mathrm{o}}\right), \partial^{2} e\left(\mathbf{p}\left(\phi_{\mathrm{o}}\right)\right) \partial_{i} \mathbf{p}\left(\theta_{\mathrm{o}}\right)\right)\right]_{1 \leq i, j \leq d-1}
\end{aligned}
$$

Because $\partial_{1} \mathbf{p}\left(\theta_{\mathrm{o}}\right), \partial_{2} \mathbf{p}\left(\theta_{\mathrm{o}}\right)$ and $\partial_{1} \mathbf{p}\left(\phi_{\mathrm{o}}\right), \partial_{2} \mathbf{p}\left(\phi_{\mathrm{o}}\right)$ span the same space, there is a nonsingular $(d-1) \times(d-1)$ matrix $U$ such that

$$
\left[\partial_{1} \mathbf{p}\left(\phi_{\mathrm{o}}\right) \partial_{2} \mathbf{p}\left(\phi_{\mathrm{o}}\right) \cdots \partial_{d-1} \mathbf{p}\left(\phi_{\mathrm{o}}\right)\right]=\left[\begin{array}{llll}
\partial_{1} \mathbf{p}\left(\theta_{\mathrm{o}}\right) & \partial_{2} \mathbf{p}\left(\theta_{\mathrm{o}}\right) & \cdots & \partial_{d-1} \mathbf{p}\left(\theta_{\mathrm{o}}\right)
\end{array}\right] U
$$

Hence the $2(d-1) \times 2(d-1)$ matrix $\partial^{2} F\left(\theta_{\mathrm{o}}, \phi_{\mathrm{o}}\right)$ blocks

$$
\partial^{2} F\left(\theta_{\mathrm{o}}, \phi_{\mathrm{o}}\right)=\left(\begin{array}{cc}
M_{\alpha}-v_{\theta} c_{\theta} M_{\theta} & v_{\theta} v_{\phi} M_{\alpha} U \\
v_{\theta} v_{\phi} U^{t} M_{\alpha} & U^{t} M_{\alpha} U-v_{\phi} c_{\phi} U^{t} M_{\phi} U
\end{array}\right)
$$

Multiplying this on the right by $\left(\begin{array}{cc}\mathbb{1} & 0 \\ 0 & U^{-1}\end{array}\right)$ and on the left by $\left(\begin{array}{cc}\mathbb{1} & 0 \\ 0 & \left(U^{t}\right)^{-1}\end{array}\right)$, we see that this has nonzero determinant if and only if

$$
\left(\begin{array}{cc}
M_{\alpha}-v_{\theta} c_{\theta} M_{\theta} & v_{\theta} v_{\phi} M_{\alpha} \\
v_{\theta} v_{\phi} M_{\alpha} & M_{\alpha}-v_{\phi} c_{\phi} M_{\phi}
\end{array}\right)
$$

has a nonzero determinant or equivalently, if and only if

$$
\begin{aligned}
\left(M_{\alpha}-v_{\theta} c_{\theta} M_{\theta}\right) x+v_{\theta} v_{\phi} M_{\alpha} y & =0 \\
v_{\theta} v_{\phi} M_{\alpha} x+\left(M_{\alpha}-v_{\phi} c_{\phi} M_{\phi}\right) y & =0
\end{aligned}
$$

has a unique solution. Solving for $y$ in the first equation, substituting in the second gives

$$
v_{\theta} v_{\phi} M_{\alpha} x-\left(M_{\alpha}-v_{\phi} c_{\phi} M_{\phi}\right) v_{\theta} v_{\phi} M_{\alpha}^{-1}\left(M_{\alpha}-v_{\theta} c_{\theta} M_{\theta}\right) x=0
$$


The matrix $M_{\alpha}$ is invertible because $S$ is strictly convex. Multiplying through by $v_{\theta} v_{\phi} M_{\alpha}^{-1}$ gives

$$
\left[\mathbb{1}-\left(\mathbb{1}-v_{\phi} c_{\phi} M_{\alpha}^{-1} M_{\phi}\right)\left(\mathbb{1}-v_{\theta} c_{\theta} M_{\alpha}^{-1} M_{\theta}\right)\right] x=0
$$

Thus $\partial^{2} F\left(\theta_{0}, \phi_{0}\right)$ has nonzero determinant if and only if

$$
\begin{aligned}
& {\left[\mathbb{1}-\left(\mathbb{1}-v_{\phi} c_{\phi} M_{\alpha}^{-1} M_{\phi}\right)\left(\mathbb{1}-v_{\theta} c_{\theta} M_{\alpha}^{-1} M_{\theta}\right)\right]} \\
& \quad=v_{\phi} c_{\phi} M_{\alpha}^{-1} M_{\phi}+v_{\theta} c_{\theta} M_{\alpha}^{-1} M_{\theta}-v_{\theta} v_{\phi} c_{\theta} c_{\phi} M_{\alpha}^{-1} M_{\theta} M_{\alpha}^{-1} M_{\phi}
\end{aligned}
$$

is invertible. By strict convexity and (B.52), $\alpha_{\mathrm{o}} \in\left\{\theta_{\mathrm{o}}, a\left(\theta_{\mathrm{o}}\right)\right\}$ and $\phi_{\mathrm{o}} \in\left\{\theta_{\mathrm{o}}, a\left(\theta_{\mathrm{o}}\right)\right\}$. If $e$ is symmetric, this implies that $M_{\alpha}=M_{\theta}=M_{\phi}$ and $c_{\theta}=c_{\phi}=1$, so the right hand side of $(B .62)$ is $\left(v_{\phi}+v_{\theta}-v_{\phi} v_{\theta}\right) \mathbb{1}$ which is invertible since the sum of three signs can never vanish. If $e$ is asymmetric, we use (H4) to say that, possibly after a change of basis (which we can do by multiplying on the right by a $V$ and on the left by its $V^{-1}$ ), each of the three matrices on the right hand side are of the form $\pm \mathbb{1}$ plus a matrix whose norm is at most $\frac{1}{5}, \frac{1}{5},\left(1+\frac{1}{5}\right)^{2}-1$, respectively, for the three matrices. As

$$
\frac{1}{5}+\frac{1}{5}+\left(1+\frac{1}{5}\right)^{2}-1=\frac{4}{5}+\frac{1}{25}<1
$$

the right hand side is invertible.

Proposition B.7 There is a constant const such that for all $d \geq 3$ and all $j_{1}, j_{2}, j_{3}<0$

$$
\begin{aligned}
\operatorname{Vol}\left\{\left(\mathbf{k}_{1}, \mathbf{k}_{2}\right) \in \mathbb{R}^{2 d}:\left|e\left(\mathbf{k}_{1}\right)\right| \leq M^{j_{1}},\left|e\left(\mathbf{k}_{2}\right)\right|\right. & \left.\leq M^{j_{2}},\left|e\left( \pm \mathbf{k}_{1} \pm \mathbf{k}_{2}+\mathbf{q}\right)\right| \leq M^{j_{3}}\right\} \\
& \leq \text { const } M^{j_{1}} M^{j_{2}} M^{j_{3}} .
\end{aligned}
$$

Proof: We may assume without loss of generality that $j_{3}=\max \left\{j_{1}, j_{2}, j_{3}\right\}$. Otherwise make a change of variables with $\mathbf{p}_{1}= \pm \mathbf{k}_{1} \pm \mathbf{k}_{2}+\mathbf{q}, \mathbf{p}_{2}=\mathbf{k}_{2}$. By compactness of a closed neighbourhood of $S$, it suffices to show that for any $\mathbf{k}_{1}^{(0)}, \mathbf{k}_{2}^{(0)}, \mathbf{q}^{(0)}$ obeying

$$
e\left(\mathbf{k}_{1}^{(0)}\right)=e\left(\mathbf{k}_{2}^{(0)}\right)=e\left( \pm \mathbf{k}_{1}^{(0)} \pm \mathbf{k}_{2}^{(0)}+\mathbf{q}^{(0)}\right)=0
$$

there is a constant const and there are neighbourhoods $V_{1}, V_{2}, U$ of $\mathbf{k}_{1}^{(0)}, \mathbf{k}_{2}^{(0)}, \mathbf{q}^{(0)}$ respectively, such that for all $\mathbf{q} \in U$ and all $j<0$

$$
\begin{gathered}
\operatorname{Vol}\left\{\left(\mathbf{k}_{1}, \mathbf{k}_{2}\right) \in V_{1} \times V_{2}:\left|e\left(\mathbf{k}_{1}\right)\right| \leq M^{j_{1}},\left|e\left(\mathbf{k}_{2}\right)\right| \leq M^{j_{2}},\left|e\left( \pm \mathbf{k}_{1} \pm \mathbf{k}_{2}+\mathbf{q}\right)\right| \leq M^{j_{3}}\right\} \\
\leq \text { const } M^{j_{1}} M^{j_{2}} M^{j_{3}}
\end{gathered}
$$

Since $M^{j_{k}}<r_{\mathrm{o}}$ for $k \in\{1,2,3\}$, we can switch to the $\rho, \theta$ coordinates. In these coordinates, the neighbourhoods $V_{1}, V_{2}$ can be replaced by some $X_{1} \times Y_{1}$ and $X_{2} \times Y_{2}$. Define

$$
E(\mathbf{q}, \theta, \phi)=e( \pm \mathbf{p}(0, \theta) \pm \mathbf{p}(0, \phi)+\mathbf{q})
$$

Since, for all $\mathbf{q}$ in a neighbourhood of $\mathbf{q}^{(0)}$ and all $(\theta, \phi) \in S^{d-1} \times S^{d-1}$,

$$
\begin{aligned}
&|E(\mathbf{q}, \theta, \phi)|= \mid e( \pm \mathbf{p}(0, \theta) \pm \mathbf{p}(0, \phi)+\mathbf{q})-e\left( \pm \mathbf{p}\left(\rho_{1}, \theta\right) \pm \mathbf{p}\left(\rho_{2}, \phi\right)+\mathbf{q}\right) \\
&\left.\quad+e\left( \pm \mathbf{p}\left(\rho_{1}, \theta\right) \pm \mathbf{p}\left(\rho_{2}, \phi\right)+\mathbf{q}\right)\right) \mid \\
& \leq \text { const }\left|\rho_{1}\right|+\text { const }\left|\rho_{2}\right|+\left|e\left( \pm \mathbf{p}\left(\rho_{1}, \theta\right) \pm \mathbf{p}\left(\rho_{2}, \phi\right)+\mathbf{q}\right)\right|,
\end{aligned}
$$


we have, for all $\mathbf{q}$ in a neighbourhood of $\mathbf{q}^{(0)}$,

$$
\begin{aligned}
& \operatorname{Vol}\left\{\left(\mathbf{k}_{1}, \mathbf{k}_{2}\right) \in V_{1} \times V_{2}:\left|e\left(\mathbf{k}_{1}\right)\right| \leq M^{j_{1}},\left|e\left(\mathbf{k}_{2}\right)\right| \leq M^{j_{2}},\left|e\left(\mathbf{k}_{1} \pm \mathbf{k}_{2}+\mathbf{q}\right)\right| \leq M^{j_{3}}\right\} \\
& \leq \text { const } \operatorname{Vol}\left\{\left(\theta, \phi, \rho_{1}, \rho_{2}\right) \in X_{1} \times X_{2} \times Y_{1} \times Y_{2}:\left|\rho_{1}\right| \leq M^{j_{1}},\left|\rho_{2}\right| \leq M^{j_{2}},\right. \\
& \left.\qquad\left|e\left(\mathbf{p}\left(\rho_{1}, \theta\right) \pm \mathbf{p}\left(\rho_{2}, \phi\right)+\mathbf{q}\right)\right| \leq M^{j_{3}}\right\} \\
& \leq \text { const } \operatorname{Vol}\left\{\left(\theta, \phi, \rho_{1}, \rho_{2}\right) \in X_{1} \times X_{2} \times Y_{1} \times Y_{2}:\left|\rho_{1}\right| \leq M^{j_{1}},\left|\rho_{2}\right| \leq M^{j_{2}},\right. \\
& \left.\qquad|E(\mathbf{q}, \theta, \phi)| \leq \mathrm{const} M^{j_{3}}\right\} \\
& \leq \text { const } M^{j_{1}+j_{2}} \operatorname{Vol}\left\{(\theta, \phi) \in X_{1} \times X_{2}:|E(\mathbf{q}, \theta, \phi)| \leq \mathrm{const} M^{j_{3}}\right\}
\end{aligned}
$$

Hence it suffices to prove that, for all $\mathbf{q}$ in a neighbourhood of $\mathbf{q}^{(0)}$,

$$
\operatorname{Vol}\left\{(\theta, \phi) \in X_{1} \times X_{2}:|E(\mathbf{q}, \theta, \phi)| \leq \text { const } M^{j}\right\} \leq \text { const } M^{j}
$$

In the event that $\left(\theta_{0}, \phi_{0}\right)$ is not a critical point of $E\left(\mathbf{q}^{(0)}, \theta, \phi\right)$, this is trivial.

So from now on suppose that $\left(\theta_{0}, \phi_{0}\right)$ is a critical point of $E\left(\mathbf{q}^{(0)}, \theta, \phi\right)$. By the previous Lemma $\partial^{2} E$ is invertible at $\left(\theta_{0}, \phi_{0}\right)$. So, by the implicit function theorem, for each $\mathbf{q}$ in a neighbourhood of $\mathbf{q}^{(0)}$

$$
\nabla E(\mathbf{q}, \theta, \phi)=0
$$

(here $\nabla$ means the derivative with respect to $\theta$ and $\phi$ ) has a unique solution $\left(\theta_{0}(\mathbf{q}), \phi_{0}(\mathbf{q})\right)$ in a neighbourhood of $\left(\theta_{0}, \phi_{0}\right)$ and this solution depends on $\mathbf{q}$ in a $C^{k-1}$ way. Consequently, $\partial^{2} E$ is also invertible at $\left(\theta_{\mathrm{o}}(\mathbf{q}), \phi_{\mathrm{o}}(\mathbf{q})\right)$. Let $d_{1}$ and $d_{2}$ be the number of positive and negative eigenvalues of $\partial^{2} E\left(\theta_{\mathrm{o}}, \phi_{\mathrm{o}}\right)$ respectively. Then $d_{1} \geq 0, d_{2} \geq 0, d_{1}+d_{2}=2(d-1) \geq 4$ since $d \geq 3$. By a translation followed by a linear change of variables we may replace $E(\mathbf{q}, \theta, \phi)$ by $F(u, v)=E(\mathbf{q}, \theta(u, v), \phi(u, v))$ with $u$ running over a neighbourhood of $0 \in \mathbb{R}^{d_{1}}, v$ running over a neighbourhood of $0 \in \mathbb{R}^{d_{2}}$ and

$$
\begin{aligned}
\partial_{u_{i}} F(0,0) & =0 \\
\partial_{v_{i}} F(0,0) & =0 \\
\partial_{u_{i}} \partial_{u_{j}} F(0,0) & =2 \delta_{i, j} \\
\partial_{v_{i}} \partial_{v_{j}} F(0,0) & =-2 \delta_{i, j} \\
\partial_{u_{i}} \partial_{v_{j}} F(0,0) & =0
\end{aligned}
$$

Note that $F(0,0)$ need not be zero.

The easy case is that with one of $d_{1}, d_{2}$ zero. Suppose that $d_{2}=0$. Then, as in Section A.2 we can make a "polar coordinate" like change of variables $u=u(r, \alpha)$, with $r$ being the square root of $F-F_{\mathrm{o}}$ and $\alpha$ being the usual polar angles. Then $f(r, \alpha)=F(u(r, \alpha))=F_{\mathrm{o}}+r^{2}$ and we are led to bound

$$
\int d \alpha \int d r r^{d_{1}+d_{2}-1} \chi\left(\left|F_{\mathrm{o}}+r^{2}\right| \leq \epsilon\right)
$$

If $F_{\mathrm{o}} \geq-2 \epsilon$, then $r \leq$ const $\sqrt{\epsilon}$ and, as $d_{1}+d_{2}-1 \geq 1$, the integral is bounded by const $\epsilon$. If $F_{\mathrm{o}}<-2 \epsilon$, then $r$ must obey $r^{2}=\left|F_{\mathrm{o}}\right|+\varepsilon$ for some $\varepsilon \in[-\epsilon, \epsilon]$. As

$$
\begin{aligned}
r & = \pm \sqrt{\left|F_{\mathrm{o}}\right|+\varepsilon} \\
& = \pm \sqrt{\left|F_{\mathrm{o}}\right|} \sqrt{1+\varepsilon /\left|F_{\mathrm{o}}\right|} \\
& = \pm \sqrt{\left|F_{\mathrm{o}}\right|}+O\left(\varepsilon / \sqrt{\left|F_{\mathrm{o}}\right|}\right)
\end{aligned}
$$


$r$ runs over an interval of length $O\left(\epsilon / \sqrt{\left|F_{\mathrm{o}}\right|}\right) \leq$ const $\sqrt{\epsilon}$ centred on $\sqrt{\left|F_{\mathrm{o}}\right|} \geq$ const $\sqrt{\epsilon}$. Hence the integral is bounded by

$$
\left|F_{\mathrm{o}}\right|^{\left(d_{1}+d_{2}-1\right) / 2} \frac{\epsilon}{\sqrt{\left|F_{\mathrm{o}}\right|}}
$$

which, in turn is bounded by const $\epsilon$, uniformly in $F_{\mathrm{o}}$ again because $d_{1}+d_{2}-1 \geq 1$.

The hard case is that with both $d_{1}$ and $d_{2}$ nonzero. Then we first go to polar coordinates in $u$ and $v$ separately.

$$
\begin{aligned}
& r=\sqrt{\sum_{i=1}^{d_{1}} u_{i}^{2}} \\
& \alpha=d_{1}-1 \text { angles for } u \\
& \rho=\sqrt{\sum_{i=1}^{d_{2}} v_{i}^{2}} \\
& \beta=d_{2}-1 \text { angles for } v
\end{aligned}
$$

The measure

$$
d^{d-1} \theta d^{d-1} \phi=\text { const } d^{d_{1}} u d^{d_{2}} v=J(\alpha, \beta) r^{d_{1}-1} \rho^{d_{2}-1} d r d \rho
$$

with $J(\alpha, \beta)$ bounded above. For each fixed $\alpha, \beta$ the function $f(r, \rho)=F(u(r, \alpha), v(\rho, \beta))$ is $C^{k}$ in $(r, \rho)$ in a neigbourhood of $(0,0) \in[0, \infty)^{2}$. Furthermore the first and second derivatives of $f$ at the origin are

$$
\begin{aligned}
f_{r}(0,0) & =0 \\
f_{\rho}(0,0) & =0 \\
f_{r r}(0,0) & =2 \\
f_{\rho \rho}(0,0) & =-2 \\
f_{r \rho}(0,0) & =0
\end{aligned}
$$

Hence there is, for $k \geq 2$, a $C^{1}$ change of variables $x=x(r, \rho), y=y(r, \rho)$ such that

$$
f(r(x, y), \rho(x, y))=F(0,0)+x^{2}-y^{2}
$$

and

$$
\frac{\partial(r, \rho)}{\partial(x, y)}(0,0)=\left(\begin{array}{ll}
1 & 0 \\
0 & 1
\end{array}\right)
$$

The latter condition ensures that

$$
\begin{aligned}
& |r| \leq a_{1}|x|+b_{1}|y| \\
& |\rho| \leq b_{2}|x|+a_{2}|y|
\end{aligned}
$$

By choosing the neighbourhood sufficiently small, the constants $a_{1}, a_{2}$ and $b_{1}, b_{2}$ can be made arbitrarily close to one and zero respectively. Hence $r^{d_{1}-1} \rho^{d_{2}-1}$ is bounded by a finite sum of terms of the form $|x|^{e_{1}}|y|^{e_{2}}$ with $e_{1}, e_{2} \geq 0, e_{1}+e_{2}=d_{1}+d_{2}-2=2 d-4$ and we are led to bound

$$
\int_{-1}^{1} d x|x|^{e_{1}} \int_{-1}^{1} d y|y|^{e_{2}} \chi\left(\left|F_{\mathrm{o}}+x^{2}-y^{2}\right| \leq \epsilon\right)
$$

with $e_{1}, e_{2} \geq 0, e_{1}+e_{2}=d_{1}+d_{2}-2=2 d-4$.

We may assume, without loss of generality that $F_{\mathrm{o}} \geq 0$. Otherwise just exchange the roles of $x$ and $y$. In the domain where $F_{\mathrm{o}}+x^{2} \leq 2 \epsilon$ both $|x|$ and $|y|$ are bounded above by $O(\sqrt{\epsilon})$. This domain domain 
contributes at most $O(\epsilon)$ to the total integral. In the domain where $F_{\mathrm{o}}+x^{2} \geq 2 \epsilon, y$ must obey $y^{2}=F_{\mathrm{o}}+x^{2}+\varepsilon$ for some $\varepsilon \in[-\epsilon, \epsilon]$. So

$$
\begin{aligned}
y & = \pm \sqrt{F_{\mathrm{o}}+x^{2}+\varepsilon} \\
& = \pm \sqrt{F_{\mathrm{o}}+x^{2}}\left(1+\frac{\varepsilon}{F_{\mathrm{o}}+x^{2}}\right)^{1 / 2} \\
& = \pm \sqrt{F_{\mathrm{o}}+x^{2}}\left(1+O\left(\frac{\epsilon}{F_{\mathrm{o}}+x^{2}}\right)\right) \\
& = \pm \sqrt{F_{\mathrm{o}}+x^{2}}+O\left(\frac{\epsilon}{\sqrt{F_{\mathrm{o}}+x^{2}}}\right)
\end{aligned}
$$

That is, $y$ runs over at most two intervals of length at most $O\left(\frac{\epsilon}{\sqrt{F_{\mathrm{o}}+x^{2}}}\right) \leq O(\sqrt{\epsilon})$ whose centers have modulus $\sqrt{F_{\mathrm{o}}+x^{2}} \geq \sqrt{2 \epsilon}$. The contribution of this domain is at most

$$
\text { const } \int d x|x|^{e_{1}}\left(\sqrt{F_{\mathrm{o}}+x^{2}}\right)^{e_{2}} \frac{\epsilon}{\sqrt{F_{\mathrm{o}}+x^{2}}}
$$

Since $e_{1}+e_{2} \geq 2$, this is bounded by $O(\epsilon)$, uniformly in $F_{0}$.

Proof of Lemma 3.9: Let $\kappa>0$. We first note that $\mathcal{P}_{\kappa}$ can be defined as in (B.11), and that Lemmas B.1 and B.2 carry over to $d \geq 3$ with trivial changes. The analogue of Lemma B.3 also holds because the matrix $\partial^{2} E$ is nonsingular by Lemma B.6, and this implies by the implicit function theorem the existence and $C^{1}$ properties of the solutions stated in $(i)$. That there are four solutions follows as in Lemma 3.2. All four solutions given in the table after (3.39) are possible because (H5) has been relaxed. Since the function is defined on the compact set $\mathcal{P}_{\kappa} \times\left([-\kappa, \kappa] \times S^{d-1}\right)^{2}$, it is uniformly $C^{1}$, so there is a global Lipschitz constant $L$ such that (3.143) holds. To prove (ii), we may assume that $\mathbf{q} \in \mathcal{P}_{\kappa}$. Let $K_{2}=L+1$ and $(\theta, \phi)$ not obey (3.145). Then for all $\left|\mathbf{q}^{\prime}-\mathbf{q}\right| \leq \varepsilon_{3}$,

$$
\begin{aligned}
\left|\theta-\theta_{c r}^{b}\left(\mathbf{q}^{\prime}\right)\right| & \geq|| \theta-\theta_{c r}^{b}(\mathbf{q})|-| \theta_{c r}^{b}\left(\mathbf{q}^{\prime}\right)-\theta_{c r}^{b}(\mathbf{q}) \| \\
& \geq K_{2} \varepsilon_{3}-L\left|\mathbf{q}^{\prime}-\mathbf{q}\right| \geq \varepsilon_{3}
\end{aligned}
$$

and similarly $\left|\phi-\phi_{c r}^{b}\left(\mathbf{q}^{\prime}\right)\right| \geq \varepsilon_{3}$ (we suppress $\rho_{\theta}$ and $\rho_{\phi}$ in the notation since everything is uniform in these variables). Thus, by Taylor expansion,

$$
\begin{aligned}
\left(\begin{array}{c}
\nabla_{\theta} E \\
\nabla_{\phi} E
\end{array}\right)\left(\mathbf{q}^{\prime}, \theta, \phi\right)=\partial^{2} E\left(\mathbf{q}^{\prime}, \theta_{c r}^{b}, \phi_{c r}^{b}\right)\left(\begin{array}{c}
\theta-\theta_{c r}^{b} \\
\phi-\phi_{c r}^{b}
\end{array}\right) & \\
& +\int_{0}^{1} d t\left(\partial^{2} E\left(\mathbf{q}^{\prime}, \theta_{c r}^{b}+t\left(\theta-\theta_{c r}^{b}\right), \phi_{c r}^{b}+t\left(\phi-\phi_{c r}^{b}\right)\right)-\partial^{2} E\left(\mathbf{q}^{\prime}, \theta_{c r}^{b}, \phi_{c r}^{b}\right)\right)\left(\begin{array}{c}
\theta-\theta_{c r}^{b} \\
\phi-\phi_{c r}^{b}
\end{array}\right)
\end{aligned}
$$

where $\theta_{c r}^{b}=\theta_{c r}^{b}\left(\mathbf{q}^{\prime}\right)$ and $\phi_{c r}^{b}=\phi_{c r}^{b}\left(\mathbf{q}^{\prime}\right)$. Since the eigenvalues of $\partial^{2} E$ are nonzero and the parameters run over a compact region, there is $K_{1}>0$ such that

$$
\left|\partial^{2} E\left(\mathbf{q}^{\prime}, \theta_{c r}^{b}, \phi_{c r}^{b}\right) v\right| \geq \frac{2}{K_{1}}|v|
$$

for all $v \in \mathbb{R}^{2(d-1)}$. By continuity, the operator norm of the matrix in the remainder term in (B.86) can be made smaller than $\frac{1}{K_{1}}$. This implies that if (3.145) does not hold, (3.144) must hold. 


\section{Appendix C. One-loop volume bounds for asymmetric Fermi surfaces}

In this appendix, we prove Lemma 4.8. We mention again that Lemma 4.8 has two important consequences - it implies differentiability of the particle-particle wicked ladder contributions to the counterterm and the self-energy (defined in (4.1) and (4.2), and drawn in Figure $6(b)$ ) in the asymmetric case (Theorem 4.9), and it also implies that the particle-particle bubble

$$
B(k, Q, q)=\lim _{I \rightarrow-\infty} \sum_{I \leq j, h<0} B_{j h}(k, Q, q)
$$

where

$$
B_{j h}(k, Q, q)=\int_{\mathbb{R} \times \mathcal{B}} d^{d+1} p C_{j}(p) \hat{v}(p-k) C_{h}(-p+Q) \hat{v}(q+p-Q)
$$

is a bounded function of the three momenta $(k, Q, q)$ if $e$ is asymmetric. If $e$ is symmetric, $B$ diverges for $|Q| \rightarrow 0$ like $\log |Q|$ for all $k, q$; this leads to the Cooper instability if the interaction is attractive. Thus Theorem 4.9 and Lemma 2.41 of I imply that no ladder subdiagrams can generate any factorials in the values of individual graphs in the perturbation expansion, if $e$ is asymmetric and if (H4') holds.

We first do some easy reduction steps. By changing variables from $\mathbf{p}$ to $\rho, \theta$, and bounding

$$
\begin{aligned}
\int d^{d} \mathbf{p} \mathbb{1}\left(|e(-\mathbf{p}+\mathbf{q})| \leq \varepsilon_{2}\right) \mathbb{1}\left(|e(\mathbf{p})| \leq \varepsilon_{1}\right) & \leq \int_{-\varepsilon_{1}}^{\varepsilon_{1}} d \rho \int d \theta J(\rho, \theta) \mathbb{1}\left(|e(-\mathbf{p}(\rho, \theta)+\mathbf{q})| \leq \varepsilon_{2}\right) \\
& \leq 2 \varepsilon_{1}|J|_{\mathrm{o}} \sup _{|\rho| \leq \varepsilon_{1}} \int d \theta \mathbb{1}\left(|e(-\mathbf{p}(\rho, \theta)+\mathbf{q})| \leq \varepsilon_{2}\right)
\end{aligned}
$$

we see that (4.21) follows from (4.20), with the constant stated in Lemma 4.8.

Since $|\rho| \leq \varepsilon_{1} \leq \varepsilon_{2}$, we can do the usual Taylor expansion

$$
\begin{aligned}
|e(-\mathbf{p}(\rho, \theta)+\mathbf{q})-e(-\mathbf{p}(0, \theta)+\mathbf{q})| & =\left|\rho \int_{0}^{1} d t \nabla e(-\mathbf{p}(t \rho, \theta)+\mathbf{q}) \cdot \partial_{\rho} \mathbf{p}(t \rho, \theta)\right| \\
& \leq \varepsilon_{1}|e|_{1}\left|\partial_{\rho} \mathbf{p}\right|_{\mathrm{o}} \leq \varepsilon_{1} \frac{|e|_{1}}{u_{\mathrm{o}}} .
\end{aligned}
$$

Here we used that, since $\rho=e(\mathbf{p}(\rho, \theta))$ and $\partial_{\rho} \mathbf{p}(\rho, \theta)=\left|\partial_{\rho} \mathbf{p}(\rho, \theta)\right| u(\mathbf{p}(\rho, \theta))$,

$$
1=\nabla e(\mathbf{p}(\rho, \theta)) \cdot \partial_{\rho} \mathbf{p}(\rho, \theta)=\nabla e(\mathbf{p}(\rho, \theta)) \cdot u(\mathbf{p}(\rho, \theta))\left|\partial_{\rho} \mathbf{p}(\rho, \theta)\right| \geq u_{\mathrm{o}}\left|\partial_{\rho} \mathbf{p}(\rho, \theta)\right|,
$$

so $\left|\partial_{\rho} \mathbf{p}\right|_{\mathrm{o}} \leq \frac{1}{u_{\mathrm{o}}}$. Therefore, for all $|\rho| \leq \varepsilon_{1},|e(-\mathbf{p}(\rho, \theta)+\mathbf{q})| \leq \varepsilon_{2}$ implies

$$
|e(-\mathbf{p}(0, \theta)+\mathbf{q})| \leq \varepsilon_{2}+\frac{|e|_{1}}{u_{\mathrm{o}}} \varepsilon_{1} \leq \varepsilon_{2}\left(1+\frac{|e|_{1}}{u_{\mathrm{o}}}\right) .
$$

Thus, if we show that there are $\alpha \geq \frac{1}{3}$ and $\tilde{Q}_{B}>0$ such that

$$
\int d \theta \mathbb{1}\left(\mid e\left(-\mathbf{p}(0, \theta)+\mathbf{q} \mid \leq \varepsilon_{2}\right) \leq \tilde{Q}_{B} \varepsilon_{2}{ }^{\alpha}\right.
$$

then (4.20) follows with $Q_{B}=\left(1+\frac{|e|_{1}}{u_{\mathrm{o}}}\right)^{\alpha} \tilde{Q}_{B}$. 


\section{C.1 Geometry of the Problem}

We now describe the geometrical picture behind the bounds. The integral in (4.21) is the $d$-dimensional volume of the set $R_{\varepsilon_{1}} \cap\left(-R_{\varepsilon_{2}}+\mathbf{q}\right) \subset \mathcal{B}$, where $R_{\varepsilon}=\{\mathbf{p}:|e(\mathbf{p})| \leq \varepsilon\}$ is a neighbourhood of the Fermi surface $S$ having thickness of order $\varepsilon>0$. From now on, we drop the subscript 2 and denote $\varepsilon_{2}=\varepsilon$. Since (C.7) holds trivially for all $\varepsilon>\varepsilon_{\mathrm{o}}$, if $\varepsilon_{\mathrm{o}}>0$ is fixed and $\tilde{Q}_{B}$ is chosen dependent on $\varepsilon_{\mathrm{o}}$, we may assume that $\varepsilon$ is small, and therefore we first draw a picture of the intersection of $S$ with its translates, in Figure 9 (one of them is drawn dotted to make the distinction easier). This corresponds to the reduction of (4.21) to (4.20). The intersection may be transversal, as in $(a)$, or tangential, as in $(b),(c)$, and $(d)$. The translating momentum $\mathbf{q}$ is $\mathbf{q}=2 \mathbf{p}$ in case $(b)$, and $\mathbf{q}=\mathbf{p}+\mathbf{a}(\mathbf{p})$ in case $(c)$ and $(d)$, where $\mathbf{p}$ is the point at which the tangential intersection takes place.

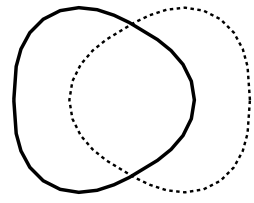

(a)

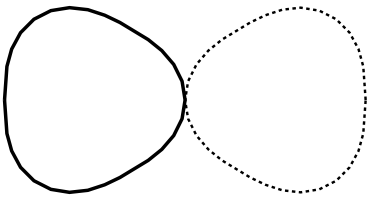

(b)

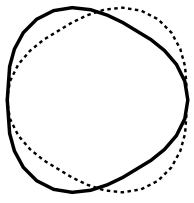

(c)

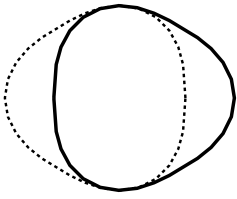

(d)

Figure 9: Intersections of $S$ and $-S+\mathbf{q}$

In Figure 10, we redraw a neighbourhood of the intersection point in coordinates where one of the surfaces appears as a straight line. In the Figure, $\mathbf{q}$ is chosen such that exact tangency happens, this is, of course, not the case in general. In fact, the coordinates used in Figure 10 are our standard coordinates $\rho$ and $\theta$, and Figure 10 simply contains the graph of the function

$$
g(\theta)=e(\mathbf{q}-\mathbf{p}(0, \theta))
$$

for four values of $\mathbf{q}$ (we suppress the dependence of $g$ on $\mathbf{q}$ in the notation because $\mathbf{q}$ is fixed). The shaded region in Figure 10 is the region where $|g| \leq \varepsilon$, in other words, it is the support of $\mathbb{1}(|g(\theta)| \leq \varepsilon)$. It is obvious from the Figure that this support condition poses a restriction on $\theta$. We now discuss briefly why this figure really captures the essential behaviour, and then turn to the details of the proof.

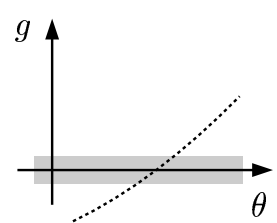

(a)

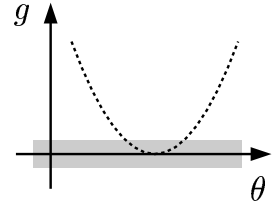

(b)

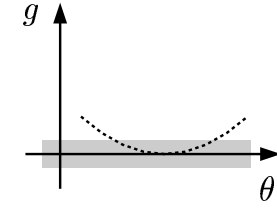

$(c)$

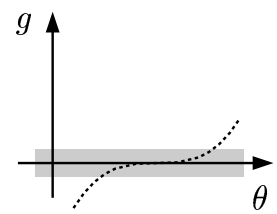

$(d)$

Figure 10: The intersection in natural coordinates 
We have

$$
\begin{aligned}
g^{\prime}(\theta) & =-\nabla e(\mathbf{q}-\mathbf{p}(0, \theta)) \cdot \partial_{\theta} \mathbf{p}(0, \theta) \\
g^{\prime \prime}(\theta) & =\left(\partial_{\theta} \mathbf{p}(0, \theta), e^{\prime \prime}(\mathbf{q}-\mathbf{p}(0, \theta)) \partial_{\theta} \mathbf{p}(0, \theta)\right)-\nabla e(\mathbf{q}-\mathbf{p}(0, \theta)) \cdot \partial_{\theta}^{2} \mathbf{p}(0, \theta)
\end{aligned}
$$

Using $\theta^{*}$ to denote the value of $\theta$ where $g(\theta)=0$, the cases drawn in the Figures are

(a) $\mathbf{q}-\mathbf{p}\left(0, \theta^{*}\right) \notin\left\{\mathbf{p}\left(0, \theta^{*}\right), \mathbf{p}\left(0, a\left(\theta^{*}\right)\right)\right\}$, so $g^{\prime}\left(\theta^{*}\right) \neq 0$. It is obvious that the condition $|g(\theta)| \leq \varepsilon$, which is the support condition of the integrand (indicated as the shaded region in Figure 10) restricts $\theta$ to an interval of length const $\varepsilon$ around $\theta^{*}$, so (C.7) holds, with $\alpha=1$.

$$
\mathbf{q}=2 \mathbf{p}\left(0, \theta^{*}\right) \text {. Then } \mathbf{q}-\mathbf{p}\left(0, \theta^{*}\right)=\mathbf{p}\left(0, \theta^{*}\right), g^{\prime}\left(\theta^{*}\right)=0 \text {, and }
$$

$$
g^{\prime \prime}\left(\theta^{*}\right)=2 w\left(\mathbf{p}\left(0, \theta^{*}\right)\right)
$$

by (2.19) and (2.24), so $\left|g^{\prime \prime}\left(\theta^{*}\right)\right| \geq 2 w_{\mathrm{o}}>0$ by (2.33). Thus the function is essentially quadratic and $|g(\theta)| \leq \varepsilon$ restricts $\theta$ to $\left|\theta-\theta^{*}\right| \leq$ const $\sqrt{\varepsilon}$, so $(C .7)$ holds with $\alpha=\frac{1}{2}$. The factor 2 in $(C .10)$ is intuitively clear from Figure $9(b)$ : the curvatures of the two intersecting sets have the same magnitudes, but opposite signs.

The other case where $g^{\prime}$ vanishes is $\mathbf{q}=\mathbf{p}\left(0, \theta^{*}\right)+\mathbf{p}\left(0, a\left(\theta^{*}\right)\right)$. Then $g^{\prime}\left(\theta^{*}\right)=0$, and by $(2.34),(2.36),(2.19)$, and (2.24),

$$
g^{\prime \prime}\left(\theta^{*}\right)=w\left(\mathbf{p}\left(0, a\left(\theta^{*}\right)\right)\right)\left(1-\frac{\partial a}{\partial \theta}\left(\theta^{*}\right)\right) .
$$

By (2.37), $\frac{\partial a}{\partial \theta}$ is the curvature ratio at $\theta^{*}$ and $a\left(\theta^{*}\right)$. This is the point where $\left(H 4^{\prime}\right)$ comes in - this ratio can equal one, and hence $g^{\prime \prime}(\theta)$ can vanish, only if $\theta^{*}$ is one of $\theta^{(1)}, \ldots, \theta^{(N)}$. So there are the cases

(c) $\quad \theta^{*} \notin U_{\delta}\left(\theta^{(1)}\right) \cup \ldots \cup U_{\delta}\left(\theta^{(N)}\right)$ for some $\delta>0$. Then $\left|\frac{\partial a}{\partial \theta}\left(\theta^{*}\right)-1\right| \geq \gamma(\delta)>0$, and the bound is as in case $(b)$, only with a $\delta$-dependent constant $1 / \gamma(\delta)$ that grows as $\delta \rightarrow 0$.

(d) $\quad \theta^{*} \in U_{\delta}\left(\theta^{(1)}\right) \cup \ldots \cup U_{\delta}\left(\theta^{(N)}\right)$. If $\theta^{*}=\theta^{(n)}$ for some $n \in\{1, \ldots, N\}, g^{\prime \prime}\left(\theta^{*}\right)=0$ because there is a tangential intersection at points with the same curvature. In a neighbourhood of these points, $\left|g^{\prime \prime}(\theta)\right|$ gets arbitrarily small. This is the hard case of the proof. Here we shall use the growth condition in $\left(H 4^{\prime}\right)$ to show that $g^{\prime \prime}$ grows at least linearly when one moves away from $\theta^{(n)}$. This will imply $(C .7)$ with $\alpha=\frac{1}{3}$.

\section{C.2 Basic properties of the critical points}

We first collect some information about those $\theta^{*}$ where $g^{\prime}\left(\theta^{*}\right)=0$ and bound the contributions to the LHS of $(C .7)$ where $\theta$ is away from these $\theta^{*}$. By Lemma B.1, if $\kappa>0$ is small enough, the equation

$$
\nabla e(\mathbf{p}(r, \vartheta)) \cdot \partial_{\theta} \mathbf{p}(0, \theta)=0
$$

has, for fixed $r$ with $|r|<\kappa$ and fixed $\theta \in \mathbb{R} / 2 \pi \mathbb{Z}$, exactly two solutions for $\vartheta$, given by $\vartheta=\vartheta^{(i)}(0, \theta, r)$, with

$$
\begin{aligned}
& \vartheta^{(1)}(0, \theta, 0)=\theta \\
& \vartheta^{(2)}(0, \theta, 0)=a(\theta),
\end{aligned}
$$


and both are $C^{1}$ functions of $(\theta, r)$. Let

$$
\mathcal{Q}_{\kappa}=\bigcup_{i=1}^{2}\left\{\mathbf{p}\left(r, \vartheta^{(i)}(0, \theta, r)\right)+\mathbf{p}(0, \theta):|r|<\kappa, \theta \in \mathbb{R} / 2 \pi \mathbb{Z}\right\}
$$

Remark C.1 There is $\kappa>0$ and $m_{\mathrm{o}}=m_{\mathrm{o}}(\kappa)>0$ such that for all $\mathbf{q} \notin \mathcal{Q}_{\kappa}$ and all $\theta \in \mathbb{R} / 2 \pi \mathbb{Z}$ satisfying $|e(\mathbf{q}-\mathbf{p}(0, \theta))| \leq \kappa / 2$,

$$
\left|\frac{\partial}{\partial \theta} e(\mathbf{q}-\mathbf{p}(0, \theta))\right| \geq m_{\mathrm{o}}(\kappa)
$$

Proof: $\quad$ The function $F:\left(\mathcal{B} \backslash \mathcal{Q}_{\kappa}\right) \times \mathbb{R} / 2 \pi \mathbb{Z} \rightarrow \mathbb{R},(\mathbf{q}, \theta) \mapsto\left|\frac{\partial}{\partial \theta} e(\mathbf{q}-\mathbf{p}(0, \theta))\right|$, is continuous. The set

$$
X_{\kappa}=\left\{(\mathbf{q}, \theta) \in\left(\mathcal{B} \backslash \mathcal{Q}_{\kappa}\right) \times \mathbb{R} / 2 \pi \mathbb{Z}:|e(\mathbf{q}-\mathbf{p}(0, \theta))| \leq \kappa / 2\right\}
$$

is compact. By construction of $X_{\kappa}, F$ has no zeros on this set, so $m_{\mathrm{o}}(\kappa)>0$ exists.

If $\mathbf{q} \notin \mathcal{Q}_{\kappa}$, then for all $\varepsilon \leq \frac{\kappa}{2}$, by a change of variables

$$
\int d \theta \mathbb{1}(|e(\mathbf{q}-\mathbf{p}(0, \theta))| \leq \varepsilon) \leq \frac{1}{m_{\mathrm{o}}(\kappa)} \int d e \mathbb{1}(|e| \leq \varepsilon)=\frac{2}{m_{\mathrm{o}}(\kappa)} \varepsilon
$$

Thus we may assume that $\mathbf{q} \in \mathcal{Q}_{\kappa}$, for some $\kappa>0$, which will be fixed, independent of $\varepsilon$, in the following.

Let $b>0$, and split

$$
\mathbb{R} / 2 \pi \mathbb{Z}=\bigcup_{n=1}^{N} U_{b}\left(\theta^{(n)}\right) \dot{\cup} \mathcal{R}_{b}
$$

(by (H4'), $b>0$ can be chosen such that $U_{b}\left(\theta^{(n)}\right) \cap U_{b}\left(\theta^{\left(n^{\prime}\right)}\right)=\emptyset$ if $\left.n \neq n^{\prime}\right)$. We fix $b>0$; then (H4') implies that there is $z_{0}>0$ such that for all $\theta \in \mathcal{R}_{b}$,

$$
\left|w(\mathbf{p}(0, a(\theta)))\left(1-\frac{\partial a}{\partial \theta}(\theta)\right)\right| \geq z_{\mathrm{o}}
$$

Lemma C.2 Define the maps $F_{1}$ and $F_{2}$ by

$$
\begin{aligned}
F_{i}: \quad(-\kappa, \kappa) \times \mathbb{R} / 2 \pi \mathbb{Z} & \rightarrow \mathcal{B} \\
(r, \theta) & \mapsto \mathbf{p}(0, \theta)+\mathbf{p}\left(r, \vartheta^{(i)}(0, \theta, r)\right) .
\end{aligned}
$$

There is $\kappa_{\mathrm{o}}=\kappa_{\mathrm{o}}(b)>0$ such that for all $0<\kappa<\kappa_{\mathrm{o}}$,

$$
\begin{aligned}
\operatorname{det} F_{1}^{\prime}(r, \theta) \neq 0 & \forall(r, \theta) \in(-\kappa, \kappa) \times \mathbb{R} / 2 \pi \mathbb{Z} \\
\operatorname{det} F_{2}^{\prime}(r, \theta) \neq 0 & \forall(r, \theta) \in(-\kappa, \kappa) \times \mathcal{R}_{b}
\end{aligned}
$$


and such that for all $\theta_{1} \in \mathbb{R} / 2 \pi \mathbb{Z}, F_{1}$ is invertible on $(-\kappa, \kappa) \times U_{\kappa}\left(\theta_{1}\right)$, and for all $\theta_{1} \in \mathcal{R}_{b}, F_{2}$ is invertible on $(-\kappa, \kappa) \times\left(U_{\kappa}\left(\theta_{1}\right) \cap \mathcal{R}_{b}\right)$.

Proof: $\quad F_{1}$ and $F_{2}$ are $C^{1}$ functions. Their derivatives, evaluated at $r=0$, are

$$
\begin{aligned}
& \frac{\partial F_{i}}{\partial r}=\partial_{\rho} \mathbf{p}\left(0, \vartheta^{(i)}(0, \theta, 0)\right)+\partial_{\theta} \mathbf{p}\left(0, \vartheta^{(i)}(0, \theta, 0)\right) \partial_{3} \vartheta^{(i)}(0, \theta, 0) \\
& \frac{\partial F_{i}}{\partial \theta}=\partial_{\theta} \mathbf{p}(0, \theta)+\partial_{\theta} \mathbf{p}\left(0, \vartheta^{(i)}(0, \theta, 0)\right) \partial_{2} \vartheta^{(i)}(0, \theta, 0) .
\end{aligned}
$$

By $(B .8)$ and (2.34),

$$
\begin{aligned}
\frac{\partial F_{1}}{\partial \theta} & =\partial_{\theta} \mathbf{p}(0, \theta)\left(1+\partial_{2} \vartheta^{(1)}(0, \theta, 0)\right) \\
\frac{\partial F_{2}}{\partial \theta} & =\partial_{\theta} \mathbf{p}(0, \theta)+\partial_{\theta} \mathbf{p}(0, a(\theta)) \partial_{2} \vartheta^{(2)}(0, \theta, 0) \\
& =\partial_{\theta} \mathbf{p}(0, \theta)\left(1-\partial_{2} \vartheta^{(2)}(0, \theta, 0)\right)
\end{aligned}
$$

so by $(B .21)$ and $(B .23)$,

$$
\begin{aligned}
& \frac{\partial F_{1}}{\partial \theta}=2 \partial_{\theta} \mathbf{p}(0, \theta) \\
& \frac{\partial F_{2}}{\partial \theta}=\partial_{\theta} \mathbf{p}(0, \theta)\left(1-\frac{\partial a}{\partial \theta}(\theta)\right) .
\end{aligned}
$$

Since $\partial_{\rho} \mathbf{p}$ and $\partial_{\theta} \mathbf{p}$ are linearly independent, $\operatorname{det} F_{i}^{\prime}(0, \theta) \neq 0$ if $\frac{\partial F_{i}}{\partial \theta} \neq 0$. Since $\left|\partial_{\theta} \mathbf{p}(0, \theta)\right|=1$ for all $\theta \in \mathbb{R} / 2 \pi \mathbb{Z}, \frac{\partial F_{1}}{\partial \theta} \neq 0$ holds. Since $\mathcal{R}_{b}$ is compact, $\frac{\partial a}{\partial \theta}$ is continuous on $\mathcal{R}_{b}$, and $\frac{\partial a}{\partial \theta}(\theta) \neq 1$ for all $\theta \in \mathcal{R}_{b}$, there is $\gamma>0$ such that

$$
\left|\frac{\partial a}{\partial \theta}(\theta)-1\right| \geq \gamma(b) \quad \forall \theta \in \mathcal{R}_{b} .
$$

By continuity of $F_{i}^{\prime}$ in $r$, there is $\tilde{\kappa}_{\mathrm{o}}(b)>0$ such that $\left|\operatorname{det} F_{i}^{\prime}(r, \theta)\right| \neq 0$ for all $r<\tilde{\kappa}_{\mathrm{o}}(b)$. By the implicit function theorem, and by compactness of $\mathbb{R} / 2 \pi \mathbb{Z}$ and $\mathcal{R}_{b}$, there is $0<\kappa_{\mathrm{o}}(b) \leq \tilde{\kappa}_{\mathrm{o}}(b)$ such that the stated local invertibility holds for all $|r|<\kappa_{\mathrm{o}}(b)$.

Remark C.3 In the corresponding two-loop statement, Lemma B.3, there are three summands, all close to \pm 1 , which can therefore never add up to zero. This is the reason why there, no restriction to a set similar to $\mathcal{R}_{b}$ was required, and why Lemma B.3 also holds in the symmetric case. Note that if (Sy) holds, then $\mathcal{R}_{b}=\emptyset$, and $\frac{\partial F_{2}}{\partial \theta} \equiv 0$ (this corresponds to $\mathbf{q}=0$ ).

Remark C.4 The local injectivity stated in Lemma C.2 does not rule out the existence of $\left(r^{\prime}, \theta^{\prime}\right)$ different from $\theta^{*}, r^{*}$, satisfying

$$
\begin{aligned}
\mathbf{q} & =\mathbf{p}\left(0, \theta^{*}\right)+\mathbf{p}\left(r^{*}, \vartheta^{(k)}\left(0, \theta^{*}, r^{*}\right)\right) \\
& =\mathbf{p}\left(0, \theta^{\prime}\right)+\mathbf{p}\left(r^{\prime}, \vartheta^{(k)}\left(0, \theta^{\prime}, r^{\prime}\right)\right)
\end{aligned}
$$

as long as $\theta^{\prime}$ and $\theta^{*}$ satisfy $\left|\theta^{*}-\theta^{\prime}\right|>\kappa$, i.e., they are far away from each other. This can happen because $\mathcal{B}$ is a torus. However, for every $\left(r^{*}, \theta^{*}\right)$ there are at most $2 \pi / \kappa \operatorname{such}\left(r^{\prime}, \theta^{\prime}\right)$ 's. (Geometrically, it is obvious that there can only be a few of them.) This complication is easily dealt with by covering the region $\mathcal{R}_{b}$ by sets with diameter at most $2 \gamma$, where $2 \gamma<\kappa$ :

$$
\mathcal{R}_{b} \subset \bigcup_{k=1}^{\bar{k}} U_{\gamma}\left(t_{k}\right) .
$$


Corollary C.5 Let $\kappa<\kappa_{0}(b)$ and fix $\mathbf{q} \in \mathcal{Q}_{\kappa}$. Then the solutions of

$$
g^{\prime}(\theta)=0, \quad \theta \in \mathcal{R}_{b}
$$

are isolated, and given by $C^{1}$ functions of $\mathbf{q}$. There is at most one solution in each $U_{\gamma}\left(t_{k}\right)$.

Proof: $\quad$ Let $\kappa<\kappa_{\mathrm{o}}(b)$ and $\mathbf{q} \in \mathcal{Q}_{\kappa}$, then

$$
\mathbf{q}=\mathbf{p}\left(0, \theta_{\mathrm{o}}\right)+\mathbf{p}\left(r_{\mathrm{o}}, \vartheta^{\left(k_{\mathrm{o}}\right)}\left(0, \theta, r_{\mathrm{o}}\right)\right)
$$

Assume that

$$
g^{\prime}\left(\theta^{*}\right)=-\nabla e\left(\mathbf{q}-\mathbf{p}\left(0, \theta^{*}\right)\right) \cdot \partial_{\theta} \mathbf{p}\left(0, \theta^{*}\right)=0 .
$$

Then by (C.12) and (C.13), there are $r^{*},\left|r^{*}\right|<\kappa$, and $k \in\{1,2\}$ with

$$
\mathbf{q}=\mathbf{p}\left(0, \theta^{*}\right)+\mathbf{p}\left(r^{*}, \vartheta^{(k)}\left(0, \theta^{*}, r^{*}\right)\right) .
$$

We cannot have

$$
\mathbf{q}=\mathbf{p}\left(0, \theta^{*}\right)+\mathbf{p}\left(r^{*}, \vartheta^{(1)}\left(0, \theta^{*}, r^{*}\right)\right)=\mathbf{p}\left(0, \theta^{* *}\right)+\mathbf{p}\left(r^{* *}, \vartheta^{(2)}\left(0, \theta^{* *}, r^{* *}\right)\right)
$$

with $\theta^{*}$ and $\theta^{* *}$ both in the same $U_{\gamma}\left(t_{k}\right)$, since this would force $\vartheta^{(1)}\left(0, \theta^{*}, 0\right)=\theta^{*}$ and $\vartheta^{(2)}\left(0, \theta^{* *}, 0\right)=a\left(\theta^{* *}\right)$ to be too close together. By injectivity, if $k=k_{\mathrm{o}}$ and $\left|\theta^{*}-\theta_{\mathrm{o}}\right|<\kappa$ then $\theta^{*}=\theta_{\mathrm{o}}$ and $r^{*}=r_{\mathrm{o}}$ must hold. So the solutions in $\theta$ are isolated, and, by $(C .21)$ and the inverse function theorem, $C^{1}$ in $\mathbf{q}$.

\section{C.3 The easy cases}

We have already dealt with the case $\mathbf{q} \notin \mathcal{Q}_{\kappa}$. By compactness, it thus suffices to consider $\mathbf{q}^{\prime} s$ in a small ball $\mathcal{B}_{\kappa} \subset \mathcal{Q}_{\kappa}$. By Corollary C.5, we may choose the $t_{k}$ 's of $(C .27)$ such that if $g^{\prime}(\theta)$ fails to have a zero in $U_{\gamma}\left(t_{k}\right)$, then $g^{\prime}(\theta)$ is bounded away from zero, uniformly for $\mathbf{q} \in \mathcal{B}_{\kappa}$. The contribution to $\int d \theta \mathbb{1}(|e(\mathbf{q}-\mathbf{p}(0, \theta))| \leq \varepsilon)$ from these $U_{\gamma}\left(t_{k}\right)$ 's is bounded as in $(C .17)$. This leaves $\mathbf{q} \in \mathcal{B}_{\kappa}, \theta$ running over $U_{\gamma}\left(t_{k}\right)$ with $k$ such that $g^{\prime}(\theta)$ vanishes at precisely one point of $U_{\gamma}\left(t_{k}\right)$ and $\mathbf{q} \in \mathcal{B}_{\kappa}, \theta$ running over $U_{\kappa}\left(\theta^{(n)}\right), 1 \leq n \leq N$. We now deal with the former case.

Let $\eta>0$. Since $g$ is continuous, $g^{-1}(]-2 \eta, 2 \eta[)$ is open, so

$$
g^{-1}(]-2 \eta, 2 \eta[)=\bigcup_{k \in \mathbb{N}}\left(a_{k}(\eta), b_{k}(\eta)\right) .
$$

The compact set $g^{-1}([-\eta, \eta])$ is also contained in this union of open intervals, hence also in a finite subcovering. Thus, choosing such a finite subcovering, relabelling (if necessary) the $k$ 's, and defining

$$
N_{\eta}=\bigcup_{k=1}^{k_{\max }}\left(a_{k}(\eta), b_{k}(\eta)\right)
$$


we have

$$
g^{-1}(]-\eta, \eta[) \subset g^{-1}([-\eta, \eta]) \subset N_{\eta} \subset g^{-1}(]-2 \eta, 2 \eta[) .
$$

That is, the set of $\theta$ where $|g(\theta)|<\eta$ is covered by finitely many open intervals with $|g(\theta)|<2 \eta$ on each interval (this is also obvious geometrically since $g^{-1}(]-\eta, \eta[$ ) is the intersection of a translate of $-S$ with a shell around $S$ ).

Let $\eta<r_{\mathrm{o}} / 2$, then we can define the map $\quad \sim$ : $N_{\eta} \rightarrow(-2 \eta, 2 \eta) \times \mathbb{R} / 2 \pi \mathbb{Z}, \theta \mapsto(\tilde{\rho}, \tilde{\theta})$ by

$$
\mathbf{q}-\mathbf{p}(0, \theta)=\mathbf{p}(\tilde{\rho}(\theta), \tilde{\theta}(\theta)),
$$

and $\tilde{\rho}, \tilde{\theta}$ are $C^{2, h}$ in $\theta$. Fix $\eta$, let $\kappa<\eta$, and let $\varepsilon<\kappa$. By definition of the coordinates $\rho$ and $\theta$,

$$
\tilde{\rho}(\theta)=e(\mathbf{p}(\tilde{\rho}, \tilde{\theta}))=e(\mathbf{q}-\mathbf{p}(0, \theta))=g(\theta)
$$

so $\tilde{\rho}=\left.g\right|_{N_{\eta}}$, and thus

$$
\int_{\mathbb{R} / 2 \pi \mathbb{Z}} d \theta \mathbb{1}(|g(\theta)| \leq \varepsilon)=\int_{N_{\eta}} d \theta \mathbb{1}(|\tilde{\rho}(\theta)| \leq \varepsilon) .
$$

Let $\theta^{*}$ obey $\frac{\partial \tilde{\rho}}{\partial \theta}\left(\theta^{*}\right)=g^{\prime}\left(\theta^{*}\right)=0$. Then, by Lemma B.1,

$$
\tilde{\theta}\left(\theta^{*}\right)=\vartheta^{(k)}\left(0, \theta^{*}, \tilde{\rho}\left(\theta^{*}\right)\right)
$$

Since $\vartheta^{(k)}$ is $C^{1}$, we may Taylor expand in $\tilde{\rho}\left(\theta^{*}\right)$ and get

$$
\tilde{\theta}\left(\theta^{*}\right)-\vartheta^{(k)}\left(0, \theta^{*}, 0\right)=\tilde{\rho}\left(\theta^{*}\right) \int_{0}^{1} d s \partial_{3} \vartheta^{(k)}\left(0, \theta^{*}, s \tilde{\rho}\left(\theta^{*}\right)\right),
$$

so that

$$
\left|\tilde{\theta}\left(\theta^{*}\right)-\vartheta^{(k)}\left(0, \theta^{*}, 0\right)\right| \leq \text { const }\left|\tilde{\rho}\left(\theta^{*}\right)\right|
$$

holds for $k \in\{1,2\}$.

We have already split the integral into contributions from $U_{\gamma}\left(t_{l}\right)$. We now only have to show that if $\theta^{*} \in U_{\gamma}\left(t_{l}\right)$, then for all $\theta \in U_{\gamma}\left(t_{l}\right), g^{\prime \prime}(\theta) \neq 0$, uniformly in $\mathbf{q}$. Thus we may assume that $\left|\theta-\theta^{*}\right| \leq \kappa$.

Let $k=1$ in $(C .38)$. Then $\vartheta^{(1)}\left(0, \theta^{*}, 0\right)=\theta^{*}$ and

$$
\begin{aligned}
|\tilde{\theta}(\theta)-\theta| & \leq\left|\tilde{\theta}(\theta)-\tilde{\theta}\left(\theta^{*}\right)\right|+\left|\tilde{\theta}\left(\theta^{*}\right)-\theta^{*}\right|+\left|\theta^{*}-\theta\right| \\
& \leq \mathrm{const}\left(\left|\tilde{\rho}\left(\theta^{*}\right)\right|+\left|\theta-\theta^{*}\right|\right) .
\end{aligned}
$$

By (2.19) and (2.24),

$$
g^{\prime \prime}(\theta)=2 w(\mathbf{p}(0, \theta))+\Phi(\theta)
$$

with

$$
\begin{aligned}
\Phi(\theta) & =\left(\partial_{\theta} \mathbf{p}(0, \theta),\left[e^{\prime \prime}(\mathbf{p}(\tilde{\rho}, \tilde{\theta}))-e^{\prime \prime}(\mathbf{p}(0, \theta)] \partial_{\theta} \mathbf{p}(0, \theta)\right)\right. \\
& -[\nabla e(\mathbf{p}(\tilde{\rho}, \tilde{\theta}))-\nabla e(\mathbf{p}(0, \theta))] \cdot \partial_{\theta}^{2} \mathbf{p}(0, \theta)
\end{aligned}
$$

Since $e \in C^{2, h}$, and by (C.40),

$$
|\Phi(\theta)| \leq \text { const }\left(\left|\tilde{\rho}\left(\theta^{*}\right)\right|+|\tilde{\rho}(\theta)|+\left|\theta-\theta^{*}\right|\right)^{h}
$$


Thus, by (2.33), if $\kappa>0$ is small enough, $g^{\prime \prime}(\theta)>0$ holds for all $\theta \in U_{\kappa}\left(\theta^{*}\right)$ with $|g(\theta)|=|\tilde{\rho}(\theta)| \leq \kappa$, and $\left|g\left(\theta^{*}\right)\right|=\left|\tilde{\rho}\left(\theta^{*}\right)\right| \leq 2 \kappa$. These conditions on $\tilde{\rho}$ are fulfilled for all $\varepsilon<\kappa$ because in the support of the integrand, $|\tilde{\rho}(\theta)| \leq \varepsilon$ and because if $\left|\tilde{\rho}\left(\theta^{*}\right)\right|>2 \kappa, g^{\prime}$ would fail to vanish on $U_{\gamma}\left(t_{l}\right)$. Note that these conditions also hold in $U_{b}\left(\theta^{(n)}\right)$ because $k=1$.

Let $k=2$ in $(C .38)$. Then $\vartheta^{(2)}\left(0, \theta^{*}, 0\right)=a\left(\theta^{*}\right)$, and therefore, as in (C.41),

$$
|\tilde{\theta}(\theta)-a(\theta)| \leq \operatorname{const}\left(\left|\tilde{\rho}\left(\theta^{*}\right)\right|+\left|\theta-\theta^{*}\right|+\left|a(\theta)-a\left(\theta^{*}\right)\right|\right) .
$$

Moreover, by $(C .9),(2.34)$, and (2.36)

$$
g^{\prime \prime}(\theta)=w(\mathbf{p}(0, a(\theta)))\left(1-\frac{\partial a}{\partial \theta}(\theta)\right)+\tilde{\Phi}(\theta)
$$

with

$$
\begin{aligned}
\tilde{\Phi}(\theta) & =\left(\partial_{\theta} \mathbf{p}(0, \theta),\left[e^{\prime \prime}(\mathbf{p}(\tilde{\rho}, \tilde{\theta}))-e^{\prime \prime}(\mathbf{p}(0, a(\theta))] \partial_{\theta} \mathbf{p}(0, \theta)\right)\right. \\
& -[\nabla e(\mathbf{p}(\tilde{\rho}, \tilde{\theta}))-\nabla e(\mathbf{p}(0, a(\theta)))] \cdot \partial_{\theta}^{2} \mathbf{p}(0, \theta) .
\end{aligned}
$$

We have

$$
|\tilde{\Phi}(\theta)| \leq \text { const }\left(\left|\tilde{\rho}\left(\theta^{*}\right)\right|+|\tilde{\rho}(\theta)|+\left|\theta-\theta^{*}\right|+\left|a(\theta)-a\left(\theta^{*}\right)\right|\right)^{h}
$$

Again, Hölder continuity and (C.19) imply that there is $\kappa>0$ such that $g^{\prime \prime}(\theta)$ is bounded away from zero, uniformly in $\mathbf{q} \in \mathcal{B}_{\kappa}$ and $\theta \in U_{\gamma}\left(t_{l}\right) \cap \mathcal{R}_{b}$.

Taylor expansion of $g^{\prime}$ gives

$$
g^{\prime}(\theta)=\left(\theta-\theta^{*}\right) \int_{0}^{1} d t g^{\prime \prime}\left(\theta^{*}+t\left(\theta-\theta^{*}\right)\right)
$$

If $\left|\theta-\theta^{*}\right|<\kappa$, the coefficient of $\theta-\theta^{*}$ is bounded away from zero, and therefore $\left|g^{\prime}(\theta)\right| \leq \varepsilon^{1 / 2}$ implies $\left|\theta-\theta^{*}\right| \leq \frac{\varepsilon^{1 / 2}}{z_{\mathrm{o}}}$. Thus

$$
\begin{aligned}
\int_{U_{\kappa}\left(t_{l}\right)} d \theta \mathbb{1}(|g(\theta)| \leq \varepsilon) & \leq \int d \theta \mathbb{1}(|g(\theta)| \leq \varepsilon) \mathbb{1}\left(\left|g^{\prime}(\theta)\right| \leq \varepsilon^{1 / 2}\right) \\
& +\int d \theta \mathbb{1}(|g(\theta)| \leq \varepsilon) \mathbb{1}\left(\left|g^{\prime}(\theta)\right| \geq \varepsilon^{1 / 2}\right) \\
& \leq \frac{2 \varepsilon^{\frac{1}{2}}}{z_{\mathrm{o}}}+\varepsilon^{-\frac{1}{2}} \int d x \mathbb{1}(|x| \leq \varepsilon) \\
& \leq 2 \varepsilon^{\frac{1}{2}}\left(1+\frac{1}{z_{\mathrm{o}}}\right),
\end{aligned}
$$

and we have proven (C.7), with $\alpha=\frac{1}{2}$, for all contributions except those from a small neighbourhood of $\theta^{(1)}, \ldots, \theta^{(N)}$. 


\section{C.4 The hard case}

We now turn to the case where a tangential intersection happens near points where $\frac{\partial a}{\partial \theta}=1$. Let $\mathbf{q}$ run over $\mathcal{B}_{\kappa}$. We have to bound the contribution from all $U_{\kappa}\left(\theta^{(n)}\right)$ to the volume integral in $(C .7)$. Let $U=U_{\kappa}\left(\theta^{(n)}\right)$ and $\varepsilon^{\prime}=\varepsilon^{\frac{2}{3}}$, then $\varepsilon^{\prime} \geq \varepsilon$. Again, we decompose

$$
\int_{U} d \theta \mathbb{1}\left(\mid g(\theta \mid \leq \varepsilon)=Z\left(\varepsilon, \varepsilon^{\prime}\right)+V\left(\varepsilon, \varepsilon^{\prime}\right)\right.
$$

where

$$
\begin{aligned}
& Z\left(\varepsilon, \varepsilon^{\prime}\right)=\int_{U \cap N_{\eta}} d \theta \mathbb{1}(|g(\theta)| \leq \varepsilon) \mathbb{1}\left(\left|g^{\prime}(\theta)\right| \leq \varepsilon^{\prime}\right) \\
& V\left(\varepsilon, \varepsilon^{\prime}\right)=\int_{U \cap N_{\eta}} d \theta \mathbb{1}(|g(\theta)| \leq \varepsilon) \mathbb{1}\left(\left|g^{\prime}(\theta)\right|>\varepsilon^{\prime}\right) .
\end{aligned}
$$

Since $\eta$ is fixed, we may assume that $\kappa$ is chosen so small that $U \cap N_{\eta}$ is a single open interval. We use that $|\tilde{\rho}| \leq \varepsilon$ must hold in the support of the integrand to replace $g^{\prime}$ by a function $f$, as follows. By $(C .35)$ and Taylor expansion in $\tilde{\rho}$,

$$
\begin{aligned}
g^{\prime}(\theta) & =-\nabla e(\mathbf{q}-\mathbf{p}(0, \theta)) \cdot \partial_{\theta} \mathbf{p}(0, \theta) \\
& =-\nabla e(\mathbf{p}(0, \tilde{\theta})) \cdot \partial_{\theta} \mathbf{p}(0, \theta)-\tilde{\rho}(\theta) \phi_{1}(\theta) \\
& =f(\theta)-g(\theta) \phi_{1}(\theta)
\end{aligned}
$$

with a function $\phi_{1}$ that is uniformly bounded on $\mathbb{R} / 2 \pi \mathbb{Z}$, and

$$
f(\theta)=-\nabla e(\mathbf{p}(0, \tilde{\theta})) \cdot \partial_{\theta} \mathbf{p}(0, \theta) .
$$

On the support of $\mathbb{1}(|g(\theta)| \leq \varepsilon)$,

$$
\left|g^{\prime}(\theta)-f(\theta)\right| \leq|g(\theta)|\left|\phi_{1}\right|_{\mathrm{o}} \leq \varepsilon\left|\phi_{1}\right|_{\mathrm{o}}
$$

so $\left|g^{\prime}(\theta)\right| \leq \varepsilon^{\prime}$ implies $|f(\theta)| \leq \varepsilon^{\prime}+\varepsilon\left|\phi_{1}\right|_{0}$, thus

$$
\mathbb{1}(|g(\theta)| \leq \varepsilon) \mathbb{1}\left(\left|g^{\prime}(\theta)\right| \leq \varepsilon^{\prime}\right) \leq \mathbb{1}(|g(\theta)| \leq \varepsilon) \quad \mathbb{1}\left(|f(\theta)| \leq \varepsilon^{\prime}\left(1+\left|\phi_{1}\right|_{0}\right)\right),
$$

and hence

$$
Z\left(\varepsilon, \varepsilon^{\prime}\right) \leq \int_{U \cap N_{\eta}} d \theta \mathbb{1}\left(\mid g(\theta \mid \leq \varepsilon) \mathbb{1}\left(|f(\theta)| \leq \varepsilon^{\prime}\left(1+\left|\phi_{1}\right|_{0}\right)\right) .\right.
$$

Conversely, if $|g(\theta)| \leq \varepsilon$ and $\left|g^{\prime}(\theta)\right| \geq \varepsilon^{\prime}$, then for all

$$
\varepsilon \leq\left(\frac{1}{2\left|\phi_{1}\right|_{0}}\right)^{3}
$$

we have

$$
|f(\theta)| \geq\left|g^{\prime}(\theta)\right|-\varepsilon\left|\phi_{1}\right|_{\mathrm{o}} \geq \frac{1}{2} \varepsilon^{\prime} .
$$

Since the constant on the right side of $(C .58)$ is fixed, we may assume that $\varepsilon$ is so small that $(C .58)$ holds.

By strict convexity, $|f(\theta)| \leq$ const $\varepsilon^{\prime}$ implies that either $|\tilde{\theta}-\theta| \leq$ const $\varepsilon^{\prime}$ or $|\tilde{\theta}-a(\theta)| \leq$ const $\varepsilon^{\prime}$. In the event that $\tilde{\theta} \approx \theta$, $\mathbf{q}$ can have no representation in the form $\mathbf{p}\left(0, \theta^{\prime}\right)+\mathbf{p}\left(r^{\prime}, \vartheta^{(2)}\left(0, \theta^{\prime}, r^{\prime}\right)\right)$ with $\theta^{\prime}$ in the 
current interval $U_{\kappa}\left(\theta^{(n)}\right)$, since $\vartheta^{(2)}\left(0, \theta^{\prime}, r^{\prime}\right) \approx a(\theta)$. This puts us into a " $g^{\prime}$ has isolated zeros" setting and is handled as in the last section.

When $\tilde{\theta}(\theta) \approx a(\theta)$, we rewrite

$$
\begin{aligned}
f(\theta) & =\nabla e(\mathbf{p}(0, \tilde{\theta})) \cdot \partial_{\theta} \mathbf{p}(0, a(\theta)) \\
& =\nabla e(\mathbf{p}(0, \tilde{\theta})) \cdot\left(\partial_{\theta} \mathbf{p}(0, a(\theta))-\partial_{\theta} \mathbf{p}(0, \tilde{\theta})\right)
\end{aligned}
$$

Taylor expansion of the second factor gives

$$
f(\theta)=(a(\theta)-\tilde{\theta}(\theta)) \Phi(\theta)
$$

with

$$
\Phi(\theta)=\int_{0}^{1} d s \partial_{\theta}^{2} \mathbf{p}(0, \tilde{\theta}+s(a(\theta)-\tilde{\theta}(\theta))) \cdot \nabla e(\mathbf{p}(0, \tilde{\theta}(\theta)))
$$

At $s=0$, the integrand is

$$
\partial_{\theta}^{2} \mathbf{p}(0, \tilde{\theta}(\theta)) \cdot \nabla e(\mathbf{p}(0, \tilde{\theta}(\theta)))=-w(\mathbf{p}(0, \tilde{\theta}(\theta)))
$$

which, by $(2.33)$, is bounded below in magnitude by $w_{\mathrm{o}}$. By continuity, $\kappa$ can be chosen so small that

$$
|\Phi(\theta)| \geq \frac{w_{\mathrm{o}}}{2}>0 .
$$

Thus

$$
Z\left(\varepsilon, \varepsilon^{\prime}\right) \leq \int_{U} d \theta \mathbb{1}(|g(\theta)| \leq \varepsilon) \mathbb{1}\left(|\tilde{\theta}(\theta)-a(\theta)| \leq \Gamma_{1} \varepsilon^{\prime}\right)
$$

with

$$
\Gamma_{1}=\frac{2}{w_{\mathrm{o}}}\left(1+\left|\phi_{1}\right|_{\mathrm{o}}\right),
$$

and it suffices to control the function $b(\theta)=a(\theta)-\tilde{\theta}(\theta)$ near its zeros to bound $Z\left(\varepsilon, \varepsilon^{\prime}\right)$.

First observe that

$$
\frac{b^{\prime}\left(\theta^{\prime}\right)-b^{\prime}(\theta)}{\theta^{\prime}-\theta}=\frac{a^{\prime}\left(\theta^{\prime}\right)-a^{\prime}(\theta)}{\theta^{\prime}-\theta}-\frac{1}{\theta^{\prime}-\theta} \int_{\theta}^{\theta^{\prime}} d s \frac{\partial^{2} \tilde{\theta}}{\partial \theta^{2}}(s)
$$

and that, by assumption $\left(H 4^{\prime}\right), \frac{a^{\prime}\left(\theta^{\prime}\right)-a^{\prime}(\theta)}{\theta^{\prime}-\theta}$ is of fixed sign and bounded below by $K_{a}$. We now show that $\frac{\partial^{2} \tilde{\theta}}{\partial \theta^{2}}$ is bounded in magnitude by $\frac{1}{2} K_{a}$. Suppose that $\mathbf{q}=\mathbf{p}\left(0, \theta^{*}\right)+\mathbf{p}\left(0, a\left(\theta^{*}\right)\right)$ with $\theta^{*}$ in the current interval $U_{\kappa}\left(\theta^{(n)}\right)$. The generalisation to $\mathbf{q}=\mathbf{p}\left(0, \theta^{*}\right)+\mathbf{p}\left(r^{*}, \vartheta^{(2)}\left(0, \theta^{*}, r^{*}\right)\right)$ with $r^{*} \neq 0$ is a small perturbation. Differentiating $(C .35)$ and recalling that $\tilde{\rho}\left(\theta^{*}\right)=0$ and $\tilde{\theta}\left(\theta^{*}\right)=a\left(\theta^{*}\right)$, we get at $\theta=\theta^{*}$

$$
-\partial_{\theta} \mathbf{p}\left(0, \theta^{*}\right)=\frac{\partial \tilde{\rho}}{\partial \theta}\left(\theta^{*}\right) \partial_{\rho} \mathbf{p}\left(0, a\left(\theta^{*}\right)\right)+\frac{\partial \tilde{\theta}}{\partial \theta}\left(\theta^{*}\right) \partial_{\theta} \mathbf{p}\left(0, a\left(\theta^{*}\right)\right) .
$$

Since $\left.\partial_{\theta} \mathbf{p}\left(0, a\left(\theta^{*}\right)\right)=-\partial_{\theta} \mathbf{p}\left(0, \theta^{*}\right)\right)$ and since $\partial_{\rho} \mathbf{p}(0, \theta)=u(\mathbf{p}(0, \theta))\left|\partial_{\rho} \mathbf{p}(0, \theta)\right|$ and $\partial_{\theta} \mathbf{p}(0, \theta)$ are linearly independent, we have

$$
\frac{\partial \tilde{\rho}}{\partial \theta}\left(\theta^{*}\right)=0, \quad \frac{\partial \tilde{\theta}}{\partial \theta}\left(\theta^{*}\right)=1 .
$$

The second derivative of $(C .35)$ at $\theta=\theta^{*}$ gives

$$
-\partial_{\theta}^{2} \mathbf{p}\left(0, \theta^{*}\right)=\frac{\partial^{2} \tilde{\rho}}{\partial \theta^{2}}\left(\theta^{*}\right) \partial_{\rho} \mathbf{p}\left(0, a\left(\theta^{*}\right)\right)+\frac{\partial^{2} \tilde{\theta}}{\partial \theta^{2}}\left(\theta^{*}\right) \partial_{\theta} \mathbf{p}\left(0, a\left(\theta^{*}\right)\right)+\partial_{\theta}^{2} \mathbf{p}\left(0, a\left(\theta^{*}\right)\right) .
$$


By $(2.36), \partial_{\theta}^{2} \mathbf{p}\left(0, a\left(\theta^{*}\right)\right) \frac{\partial a}{\partial \theta}\left(\theta^{*}\right)=-\partial_{\theta}^{2} \mathbf{p}\left(0, \theta^{*}\right)$ and

$$
0=\frac{\partial^{2} \tilde{\rho}}{\partial \theta^{2}}\left(\theta^{*}\right) \partial_{\rho} \mathbf{p}\left(0, a\left(\theta^{*}\right)\right)+\frac{\partial^{2} \tilde{\theta}}{\partial \theta^{2}}\left(\theta^{*}\right) \partial_{\theta} \mathbf{p}\left(0, a\left(\theta^{*}\right)\right)+\left(1-\frac{\partial a}{\partial \theta}\left(\theta^{*}\right)\right) \partial_{\theta}^{2} \mathbf{p}\left(0, a\left(\theta^{*}\right)\right) .
$$

Recalling that $\left(\partial_{\theta} \mathbf{p}\right)^{2}=1$ implies that $\partial_{\theta}^{2} \mathbf{p}$ is orthogonal to $\partial_{\theta} \mathbf{p}$, we dot with $\partial_{\theta} \mathbf{p}$ to get

$$
\frac{\partial^{2} \tilde{\theta}}{\partial \theta^{2}}\left(\theta^{*}\right)=-\partial_{\rho} \mathbf{p}\left(0, a\left(\theta^{*}\right)\right) \cdot \partial_{\theta} \mathbf{p}\left(0, a\left(\theta^{*}\right)\right) \frac{\partial^{2} \tilde{\rho}}{\partial \theta^{2}}\left(\theta^{*}\right)
$$

Substituting this in and dotting instead with $\partial_{\rho} \mathbf{p}\left(0, a\left(\theta^{*}\right)\right)$ then yields

$$
\begin{aligned}
& \frac{\partial^{2} \tilde{\rho}}{\partial \theta^{2}}\left(\theta^{*}\right)=\left(1-\frac{\partial a}{\partial \theta}\left(\theta^{*}\right)\right) X_{\rho}\left(\theta, \theta^{*}\right) \\
& \frac{\partial^{2} \tilde{\theta}}{\partial \theta^{2}}\left(\theta^{*}\right)=\left(1-\frac{\partial a}{\partial \theta}\left(\theta^{*}\right)\right) X_{\theta}\left(\theta, \theta^{*}\right)
\end{aligned}
$$

where

$$
\begin{aligned}
& X_{\rho}\left(\theta, \theta^{*}\right)=-\frac{\partial_{\theta}^{2} \mathbf{p}\left(0, a\left(\theta^{*}\right)\right) \cdot \partial_{\rho} \mathbf{p}\left(0, a\left(\theta^{*}\right)\right)}{\left(\partial_{\rho} \mathbf{p}\left(0, a\left(\theta^{*}\right)\right)\right)^{2}-\left[\partial_{\rho} \mathbf{p}\left(0, a\left(\theta^{*}\right)\right) \cdot \partial_{\theta} \mathbf{p}\left(0, a\left(\theta^{*}\right)\right)\right]^{2}} \\
& X_{\theta}\left(\theta, \theta^{*}\right)=-\partial_{\rho} \mathbf{p}\left(0, a\left(\theta^{*}\right)\right) \cdot \partial_{\theta} \mathbf{p}\left(0, a\left(\theta^{*}\right)\right) X_{\rho}\left(\theta, \theta^{*}\right)
\end{aligned}
$$

are bounded $C^{0, h}$ functions (because the change of variables is regular and $\partial_{\theta} \mathbf{p}$ is a unit vector the denominator is bounded below by a fixed positive number). As $\frac{\partial a}{\partial \theta}\left(\theta^{(n)}\right)=1$, we conclude that, if $\kappa$ is small enough, $\frac{\partial^{2} \tilde{\theta}}{\partial \theta^{2}}$ is bounded above in magnitude by $\frac{1}{2} K_{a}$ and consequently $\frac{b^{\prime}\left(\theta^{\prime}\right)-b^{\prime}(\theta)}{\theta^{\prime}-\theta}$ is of fixed sign and magnitude at least $\frac{1}{2} K_{a}$.

We are now in a position to bound $Z\left(\varepsilon, \varepsilon^{\prime}\right)$.

$$
\begin{aligned}
Z\left(\varepsilon, \varepsilon^{\prime}\right) & \leq \int_{U} d \theta \mathbb{1}\left(|b(\theta)| \leq \Gamma_{1} \varepsilon^{\prime}\right) \\
& \leq \int_{U} d \theta \mathbb{1}\left(|b(\theta)| \leq \Gamma_{1} \varepsilon^{\prime}\right) \mathbb{1}\left(\left|b^{\prime}(\theta)\right| \leq \sqrt{\Gamma_{1} \varepsilon^{\prime}}\right)+\int_{U} d \theta \mathbb{1}\left(|b(\theta)| \leq \Gamma_{1} \varepsilon^{\prime}\right) \mathbb{1}\left(\left|b^{\prime}(\theta)\right| \geq \sqrt{\Gamma_{1} \varepsilon^{\prime}}\right) \\
& \leq \int_{U} d \theta \mathbb{1}\left(\left|b^{\prime}(\theta)\right| \leq \sqrt{\Gamma_{1} \varepsilon^{\prime}}\right)+\int_{U} d \theta \mathbb{1}\left(|b(\theta)| \leq \Gamma_{1} \varepsilon^{\prime}\right) \mathbb{1}\left(\left|b^{\prime}(\theta)\right| \geq \sqrt{\Gamma_{1} \varepsilon^{\prime}}\right) \\
& \leq \frac{4}{K_{a}} \sqrt{\Gamma_{1} \varepsilon^{\prime}}+\int_{U} d \theta \mathbb{1}\left(|b(\theta)| \leq \Gamma_{1} \varepsilon^{\prime}\right) \mathbb{1}\left(\left|b^{\prime}(\theta)\right| \geq \sqrt{\Gamma_{1} \varepsilon^{\prime}}\right)
\end{aligned}
$$

As $b^{\prime}(\theta)$ is monotone, $b(\theta)$ can have at most two zeros so

$$
\int_{U} d \theta \mathbb{1}\left(|b(\theta)| \leq \Gamma_{1} \varepsilon^{\prime}\right) \mathbb{1}\left(\left|b^{\prime}(\theta)\right| \geq \sqrt{\Gamma_{1} \varepsilon^{\prime}}\right) \leq 4 \frac{\Gamma_{1} \varepsilon^{\prime}}{\sqrt{\Gamma_{1} \varepsilon^{\prime}}}
$$

and $Z\left(\varepsilon, \varepsilon^{\prime}\right) \leq 4\left(1+\frac{1}{K_{a}}\right) \sqrt{\Gamma_{1} \varepsilon^{\prime}} \leq 4\left(1+\frac{1}{K_{a}}\right) \sqrt{\Gamma_{1}} \varepsilon^{\frac{1}{3}}$.

Finally, we turn to $V\left(\varepsilon, \varepsilon^{\prime}\right)$. By $(C .59)$,

$$
V\left(\varepsilon, \varepsilon^{\prime}\right) \leq \int_{U \cap N_{\eta}} d \theta \mathbb{1}(|g(\theta)| \leq \varepsilon) \mathbb{1}\left(\left|g^{\prime}(\theta)\right|>\varepsilon^{\prime}\right) \mathbb{1}\left(|f(\theta)| \geq \frac{1}{2} \varepsilon^{\prime}\right) .
$$

Denoting $|\Phi|_{\mathrm{o}}=W_{1}$, we have by $(C .61)$ that $|f(\theta)| \geq \frac{1}{2} \varepsilon^{\prime}$ implies $|\tilde{\theta}(\theta)-a(\theta)| \geq \frac{1}{2 W_{1}} \varepsilon^{\prime}$. We have just seen that $f$ has only two zeros on $U \cap N_{\eta}$ that decompose $U \cap N_{\eta}$ into at most three intervals. By (C.59) and 
continuity of $g^{\prime}, g$ is monotonic on each of these intervals. Therefore, changing variables from $\theta$ to $\gamma=g(\theta)$ on each of these intervals, and noting that the Jacobian is bounded by $\frac{2 W_{1}}{\varepsilon^{\prime}}$, we have

$$
V\left(\varepsilon, \varepsilon^{\prime}\right) \leq 3 \frac{2 W_{1}}{\varepsilon^{\prime}} \int d \gamma \mathbb{1}(|\gamma| \leq \varepsilon) \leq 12 W_{1} \frac{\varepsilon}{\varepsilon^{\prime}}=12 W_{1} \varepsilon^{\frac{1}{3}} .
$$

We have thus proven $(C .7)$ with $\alpha=\frac{1}{3}$.

\section{Appendix D. Properties of the Scale Zero Effective Action}

In this appendix, we prove Lemma 2.3. The scale zero part of the propagator $C_{\mathrm{o}}$ is a bounded function because the function $a$ cuts off values of the denominator smaller than $M^{-2}$. Since $C_{\mathrm{o}} \sim \frac{1}{i p_{\mathrm{o}}}$ for large $p_{\mathrm{o}}$, it is obvious that the integral over $\left|C_{\mathrm{o}}\right|^{n}$ is finite for all $n \geq 2$. Consequently, the most delicate part of the proof of Lemma 2.3 is the proof that $\int C_{\mathrm{o}}$ and $\int d p \hat{v}(q-p) C_{\mathrm{o}}(p)$ converge. These integrals correspond to graphs with a loop containing only one fermion propagator. They are, actually, the first order graphs shown in Figure 1, but with $C_{\mathrm{o}}$ as the fermion propagator. As discussed, for $n=1$, the integral converges at large $p_{\mathrm{o}}$ only because of sign cancellations that come from the boundary value prescription (2.44). The latter implies by a contour integral argument that for the propagator without any cutoff,

$$
\int d^{d+1} p C\left(p_{0}, e(\mathbf{p})\right)=\int d^{d} \mathbf{p} \mathbb{1}(e(\mathbf{p})<0) .
$$

A similar contour integral argument for the first order $\int \hat{v}(q-p) C(p) d^{d+1} p$ requires some analyticity properties of $\hat{v}$. The cutoff function $a$ appearing in the definition of $C_{\mathrm{o}}$ cannot be analytic in any neighbourhood of $\mathbb{R}$, so the above contour argument does not apply to $C_{0}$. We show convergence of the integrals at large $p_{\mathrm{o}}$ by a different argument which does not use any analyticity properties. This is of course possible since the convergence of the integral only depends on properties of $C_{\mathrm{o}}$ on the real line, and since the function $a$ does not change the behaviour for large $p_{0}$. One advantage of this proof is that it applies to all $\hat{v}$ satisfying (H1), which is a rather general class of potentials (in particular, no properties of $\hat{v}$ for $p_{\mathrm{o}}$ away from the real axis are required at all.

Lemma D.1 Assume that $(\mathrm{H} 1)_{k, h}$ and $(\mathrm{H} 2)_{k, h}$ hold, and let $C_{\mathrm{o}}$ be given by (2.47). Then the function

$$
I(q)=\int_{\mathbb{R} \times \mathcal{B}} \hat{v}(q-p) C_{\mathrm{o}}\left(p_{\mathrm{o}}, e(\mathbf{p})\right) d^{d+1} p=\lim _{\tau \downarrow 0} \int_{\mathbb{R} \times \mathcal{B}} \hat{v}(q-p) \frac{a\left(p_{\mathrm{o}}^{2}+e(\mathbf{p})^{2}\right)}{i p_{\mathrm{o}}-e(\mathbf{p})} e^{i p_{\mathrm{o}} \tau} d^{d+1} p
$$

is finite, uniformly bounded, and $C^{k, h}$.

Proof: We do the case $\hat{v}$ independent of $q_{\mathrm{o}}$ first. The integrand is bounded because of the cutoff function a. We do the $p_{\mathrm{o}}$-integration first. Fix $\mathbf{p} \in \mathcal{B}$, and let $e=e(\mathbf{p})$. We show that

$$
\lim _{\tau \downarrow 0} \lim _{A \rightarrow-\infty} \lim _{B \rightarrow \infty} \int_{A}^{B} C_{\mathrm{o}}\left(p_{\mathrm{o}}, e\right) e^{i \mathbf{p}_{\mathrm{o}} \tau} d p_{\mathrm{o}}
$$


converges. It is obvious that the integral from -1 to 1 converges uniformly in $\tau$ since the function $a$ makes $C_{\mathrm{o}}$ bounded. Thus we may assume $A<-1, B>1$, and we have to show that the integrals over $[A,-1]$ and $[1, B]$ converge. Both contributions are similar, so we consider only the second one. Using

$$
\frac{1}{i p_{\mathrm{o}}-e}-\frac{1}{i p_{\mathrm{o}}}=-\frac{e}{p_{\mathrm{o}}^{2}+i e p_{\mathrm{o}}}
$$

we have

$$
\int_{1}^{B} d p_{\mathrm{o}} \frac{a\left(p_{\mathrm{o}}^{2}+e(\mathbf{p})^{2}\right)}{i p_{\mathrm{o}}-e(\mathbf{p})} e^{i p_{\mathrm{o}} \tau}=\int_{1}^{B} d p_{\mathrm{o}} \frac{a\left(p_{\mathrm{o}}^{2}+e(\mathbf{p})^{2}\right)}{i p_{\mathrm{o}}} e^{i p_{\mathrm{o}} \tau}-\tilde{I}(\tau)
$$

The integrand in $\tilde{I}(\tau)$ is bounded by $\frac{\bar{e}}{p_{\mathrm{o}}^{2}} \mathbb{1}\left(\left|p_{\mathrm{o}}\right| \geq 1\right.$ ) (here $\bar{e}=\max _{\mathbf{p} \in \mathcal{B}} e(\mathbf{p})$ ), so the integral is absolutely convergent, uniformly in $\tau$, to a function that is continuous in $\tau$. Hence we only have to show that $\int_{1}^{B} \frac{d p_{0}}{i p_{\mathrm{o}}} e^{i p_{\mathrm{o}} \tau}$ converges. The convergence of this integral at fixed $\tau$ is now a consequence of

$$
\int_{1}^{B} \frac{d p_{\mathrm{o}}}{i p_{\mathrm{o}}} e^{i p_{\mathrm{o}} \tau}=\int_{1}^{B} \frac{d p_{\mathrm{o}}}{i p_{\mathrm{o}}} \frac{1}{i \tau} \frac{d}{d p_{\mathrm{o}}} e^{i p_{\mathrm{o}} \tau}=-\left.\frac{e^{i p_{\mathrm{o}} \tau}}{p_{\mathrm{o}} \tau}\right|_{1} ^{B}-\int_{1}^{B} \frac{d p_{\mathrm{o}}}{\tau p_{\mathrm{o}}^{2}} e^{i p_{\mathrm{o}} \tau}
$$

Although the above bounds are not uniform in $\tau$, they show that at fixed $\tau$, the limit

$$
J(\tau)=\lim _{A \rightarrow-\infty, B \rightarrow \infty} \int_{A}^{B} d p_{\mathrm{o}} \frac{a\left(p_{\mathrm{o}}^{2}+e(\mathbf{p})^{2}\right)}{i p_{\mathrm{o}}-e(\mathbf{p})} e^{i p_{\mathrm{o}} \tau}
$$

exists. Thus we may calculate it by taking $B=L, A=-L$. Since

$$
\begin{array}{r}
\int_{-L}^{-1} d p_{\mathrm{o}} \frac{e^{i p_{\mathrm{o}} \tau}}{i p_{\mathrm{o}}}+\int_{1}^{L} d p_{\mathrm{o}} \frac{e^{i p_{\mathrm{o}} \tau}}{i p_{\mathrm{o}}}=2 \int_{1}^{L} d p_{\mathrm{o}} \frac{\sin \left(p_{\mathrm{o}} \tau\right)}{p_{\mathrm{o}}}=2 \int_{\tau}^{L \tau} d x \frac{\sin x}{x} \\
\underset{L \rightarrow \infty}{\longrightarrow} 2 \int_{\tau}^{L \tau} d x \frac{\sin x}{x} \underset{\tau \rightarrow 0}{\longrightarrow} 2 \int_{0}^{\infty} d x \frac{\sin x}{x}=\pi,
\end{array}
$$

we see that $J(\tau)$ converges as $\tau \rightarrow 0$.

If $\hat{v}$ is not constant, but obeys (H1), we write the integral as a sum of ones with the $p_{\mathrm{o}}$-independent constant $\tilde{v}(\mathbf{q}-\mathbf{p})$ and the difference $\hat{v}\left(q_{\mathrm{o}}-p_{\mathrm{o}}, \mathbf{q}-\mathbf{p}\right)-\tilde{v}(\mathbf{q}-\mathbf{p})$. The decay assumed in (H1) makes the second integral convergent and bounded uniformly in $q_{\mathrm{o}}$. The first one was treated above and is independent of $q_{\mathrm{o}}$. Convergence of the integral for the derivatives of $I$ is even easier: apply integration by parts to move one derivative to $C_{\mathrm{o}}$, then the integral converges absolutely.

The remaining integral over $\mathbf{p}$ is over a compact region, so its convergence is trivial since the integrand is bounded.

Proof of Lemma 2.3: The kernels $V_{m, r}^{(0)}$ of the scale zero effective actions are sums over values of Feynman graphs, with propagator $C_{\mathrm{o}}$ and vertex function $\hat{v}$. The vertex function $\hat{v}$ is bounded. If we slice

$$
C_{\mathrm{o}}\left(p_{\mathrm{o}}, e(\mathbf{p})\right)=\sum_{n=1}^{\infty} C_{n}\left(p_{\mathrm{o}}, e(\mathbf{p})\right)
$$


with $C_{n}\left(p_{\mathrm{o}}, e(\mathbf{p})\right)$ having $p_{\mathrm{o}}$ support contained in $\left[M^{n}, M^{n+2}\right]$ for $n \geq 2$, and $p_{\mathrm{o}}$ support contained in $\left[0, M^{3}\right]$ for $n=1$, then

$$
\begin{aligned}
\sup \left|C_{n}\right| & \leq \text { const } M^{-n} \\
\text { vol } \operatorname{supp} C_{n} & \leq \text { const } M^{n}
\end{aligned}
$$

Consequently, the degree of any graph $G$ is

$$
\begin{aligned}
& -\mid\{\text { lines of } G\}|+|\{\text { loops of } G\} \mid= \\
& -\mid\{\text { lines of } G\} \mid+(\mid\{\text { lines of } G\}|-|\{\text { vertices of } G\} \mid+1) \\
& =1-\mid\{\text { vertices of } G\} \mid
\end{aligned}
$$

which is convergent for all graphs having strictly more than one vertex. The graphs with only one vertex were treated in Lemma D.1. Lemma 2.3 now follows by the standard power counting bounds (see, e.g. I, Theorem 2.40). 


\section{References}

[BG] G. Benfatto, G. Gallavotti, Perturbation Theory of the Fermi Surface in a Quantum Liquid. A General Quasiparticle Formalism and One-Dimensional Systems, J. Stat. Phys. 59 (1990) 541

[BGPS] G. Benfatto, G. Gallavotti, A. Procacci, B. Scoppola, Beta Function and Schwinger functions for a Many-Fermion System in One Dimension. Anomaly of the Fermi Surface, Comm. Math. Phys. 160 (1994) 93

[BM] F. Bonetto, V. Mastropietro, Beta Function and Anomaly of the Fermi Surface for a $d=1$ System of Interacting Fermions in a Periodic Potential, Comm. Math. Phys. 172 (1995) 57

[F] S. Fujimoto, Anomalous Damping of Quasiparticles in Two-Dimensional Fermi Systems, Journal of the Physical Society of Japan 60(1991) 2013

[FKLT] J. Feldman, H. Knörrer, D. Lehmann, E. Trubowitz, Fermi Liquids in Two Space Dimensions, in Constructive Physics, V. Rivasseau (ed.), Springer Lecture Notes in Physics, 1995

[FMRT] J.Feldman, J.Magnen, V.Rivasseau, E.Trubowitz, An Infinite Volume Expansion for Many Fermion Green's Functions, Helvetica Physica Acta 65 (1992) 679

[FST] J. Feldman, M. Salmhofer and E. Trubowitz, Perturbation Theory around Non-Nested Fermi Surfaces I. Keeping the Fermi Surface Fixed, Jour. Stat. Phys. 84 (1996) 1209

[FT1] J.Feldman and E.Trubowitz, Perturbation Theory for Many-Fermion Systems, Helvetica Physica Acta 63 (1990) 156

[FT2] J.Feldman and E.Trubowitz, The Flow of an Electron-Phonon System to the Superconducting State, Helvetica Physica Acta 64 (1991) 213

[GN] G. Gallavotti and F. Nicolò, Renormalization Theory in Four Dimensional Scalar Fields, Comm. Math. Phys. 100 (1985) 545 and 101 (1985) 247

[MW] J. Mawhin and M. Willem, Critical Point Theory and Hamiltonian Systems, Springer Applied Mathematical Sciences, vol. 74, Springer 1989

[S] M. Salmhofer, Improved Power Counting and Fermi Surface Renormalization, cond-mat/9607022 